University of Louisville ThinkIR: The University of Louisville's Institutional Repository

Electronic Theses and Dissertations

$5-2019$

\title{
Doing place : a framework for interpreting young adults commitments to Appalachian Kentucky.
}

Jeanelle Susanne Sears

University of Louisville

Follow this and additional works at: https://ir.library.louisville.edu/etd

Part of the Social Work Commons

\section{Recommended Citation}

Sears, Jeanelle Susanne, "Doing place : a framework for interpreting young adults' commitments to Appalachian Kentucky." (2019). Electronic Theses and Dissertations. Paper 3225.

https://doi.org/10.18297/etd/3225

This Doctoral Dissertation is brought to you for free and open access by ThinkIR: The University of Louisville's Institutional Repository. It has been accepted for inclusion in Electronic Theses and Dissertations by an authorized administrator of ThinkIR: The University of Louisville's Institutional Repository. This title appears here courtesy of the author, who has retained all other copyrights. For more information, please contact thinkir@louisville.edu. 
DOING PLACE:

A FRAMEWORK FOR INTERPRETING YOUNG ADULTS' COMMITMENTS TO APPALACHIAN KENTUCKY

By

Jeanelle Susanne Sears

B.A., University of Louisville, 2005

MSc., Brunel University, 2009

MSW, University of Vermont, 2011

\section{A Dissertation}

Submitted to the Faculty of the Raymond A. Kent School of Social Work of The University of Louisville in Partial Fulfillment of the

Requirements for the Degree of

Doctor of Philosophy

In Social Work

Raymond A. Kent School of Social Work

University of Louisville

Louisville, Kentucky

May, 2019 
(C) Copyright by

Jeanelle Susanne Sears

2019 

DOING PLACE:

A FRAMEWORK FOR INTERPRETING YOUNG ADULTS' COMMITMENTS TO APPALACHIAN KENTUCKY

By

Jeanelle Susanne Sears

B.A., University of Louisville, 2005

MSc., Brunel University, 2009

MSW, University of Vermont, 2011

A Dissertation Approved on

April 12, 2019

By the Following Dissertation Committee:

(Thomas Lawson) Co-chair

(Lesley Harris) Co-chair

(Martin Hall)

(Hee Hyul Moon)

(Marie-Antoinette Sossou) 
DEDICATION

-For Lucy 


\section{ACKNOWLEDGEMENTS}

First and foremost, I must thank the individuals who generously gave their time to participate in this study. Holding their stories is a great honor, as is witnessing and enjoying all the brilliant fruits of their labor. I am, as ever, in awe of their commitments and proud to call Appalachian Kentucky home.

During my time at the Kent School of Social Work, the faculty and staff have been critical to my success. Dr. Bibhuti Sar and Norma Kyriss, in particular provided plentiful help along the way including by helping me secure financial support for my studies and conference travel. And the Ruth Huber Scholarship provided critical funds for data collection. In this regard, I am also grateful to the School of Interdisciplinary and Graduate Studies' for their Dissertation Completion Award. This generous scholarship allowed me to dedicate the final months solely to completing this project in a timely and thoughtful way.

Dr. Martin Hall also deserves my most sincere gratitude as he served as a bighearted mentor throughout my time at Kent. He generously provided a research assistantship and opportunities for collaboration, but also sage advice and guidance. A fellow Appalachian, he also served as a valued member of my dissertation committee. Joining Dr. Hall on my committee are Drs. Hee Hyul Moon and Marie- Antoinette Sossou, each of whom brought unique insight into this work, but also supported me through opportunities for research collaboration and independent study. For the dissertation, however, I am most indebted to my co-chairs Drs. Thomas Lawson and Lesley Harris. Through a most unique combination of patience and tough 
love, Dr. Lawson helped me to articulate my vision for this project and to jump multiple hurdles along the way. Dr. Harris offered her own brand of support balancing high expectations, fierce attention to detail, and compassion as I crafted my methods and results. This work is stronger for this I am earnestly grateful for the lessons learned.

Completing the doctoral dissertation is just the final step in a long journey; one that took me 18 years and crossed four continents. At every stage, I dedicated myself to my work while navigating many other life events; both challenging and exciting. But doing so would not have been possible without the support of three strong Appalachian women. I want to thank mommaw for nurturing my heart for culture and place and guiding my hands in service. My mom too has maintained relentless support and made many sacrifices for me to get here. I especially want to thank her for bravely letting me travel far while always having a place for me to return. To my sister, I am indebted for nearly two decades of pro bono processing and grateful for many, many laughs along the way.

Finally, my partner Jordan deserves my deepest gratitude for his precious and unwavering devotion to my success in this and all aspects our life together. He has also politely endured the inelegance of a home decorated with flip chart papers. I am so thankful to be doing place with him by my side. 


\section{ABSTRACT \\ DOING PLACE: \\ A FRAMEWORK FOR INTERPRETING YOUNG ADULTS' COMMITMENTS TO APPALACHIAN KENTUCKY}

Jeanelle S. Sears

April 12, 2019

Confronted with negative consequences of rural youth outmigration, many rural communities are interested in how to recruit and retain young adults. Yet, attention has largely been focused on those who leave. This study thus explores the experiences of young adults who make conscious commitments to improving the communities of Appalachian Kentucky despite many obstacles and pressures to leave. Two aims were established in this pursuit: 1) Understand and provide deep description of young adults' experience of committing to stay and work in Appalachian Kentucky and the meaning they make of these decisions and 2) Develop a context specific framework for interpretation of these decisions and processes as grounded in participant experience.

Questions supporting and guiding these aims included: 1) What processes inform young adults' decisions to live and work in Appalachian Kentucky? 2) What strategies do young adults engage to maintain these commitments? And 3) How do young adults make meaning of these commitments? The nature of these questions warranted a qualitative approach designed to gain in depth understanding from individuals who have directly encountered these decisions and processes (Padgett, 2008). This study aimed at such an understanding by focusing on college educated young adults living in Appalachian Kentucky and working on social, environmental, 
and economic issues therein. The overall approach to the study was guided by Constructivist Grounded Theory, which included methods of participant observation, document review, and in-depth interviews with 30 young adults. Results include the development of three prominent processes identified in response to each study question, respectively: working out place, keeping peace in place, and connecting to the big picture. Combining these, a context specific framework titled doing place was developed to illustrate the dynamic process through which young adults came to understand, engage with, and ultimately transform Appalachian Kentucky. 


\section{TABLE OF CONTENTS}

DEDICATION

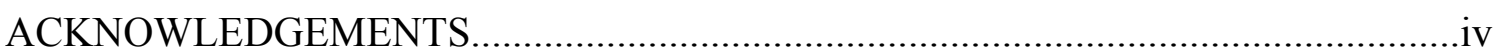

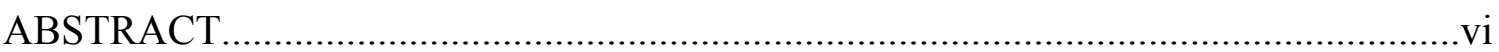

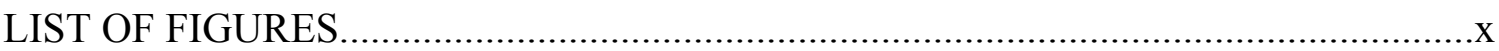

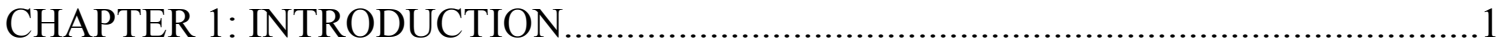

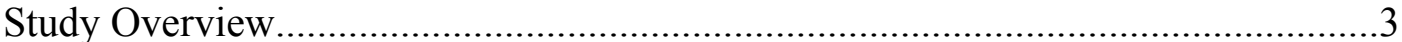

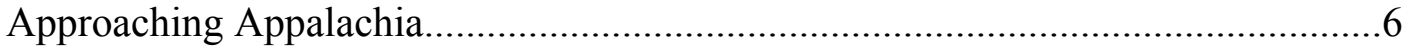

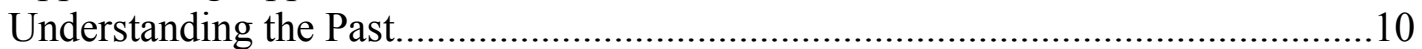

Contextualizing the Present.................................................................................... 17

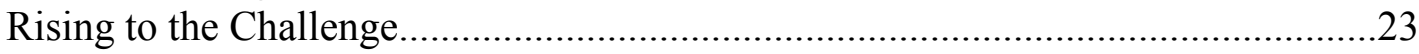

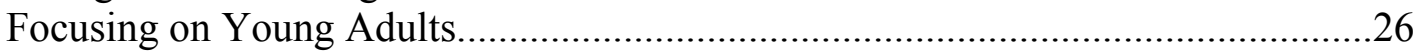

Establishing Significance to Social Work.............................................................. 30

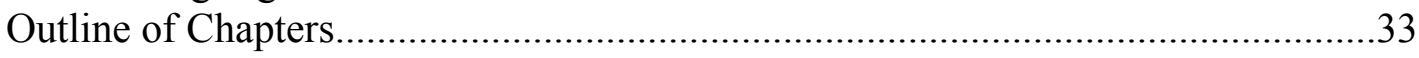

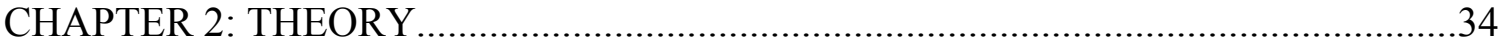

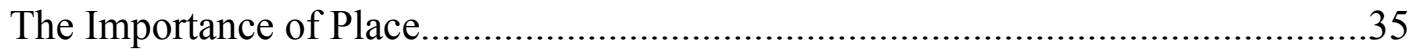

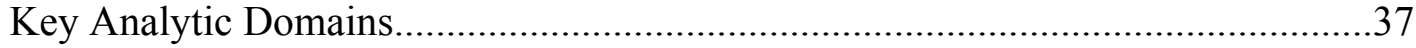

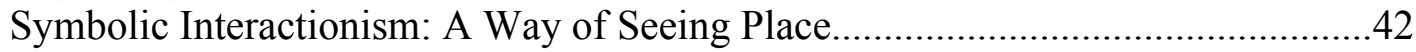

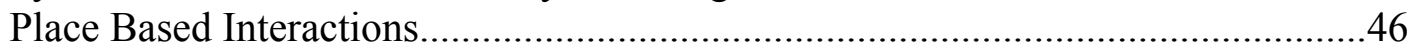

Synthesizing a Theoretical Approach to Place.....................................................50

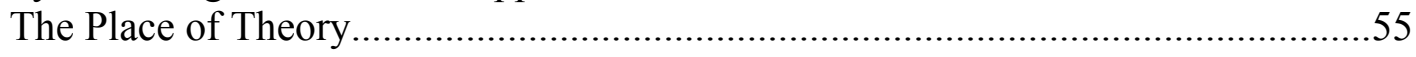

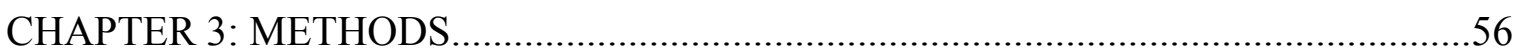

Constructivist Grounded Theory.........................................................................56

Epistemological, Ontological, and Theoretical Foundations..................................57

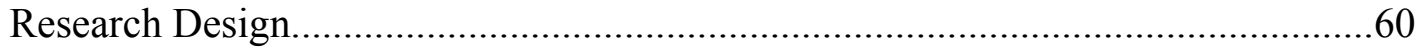

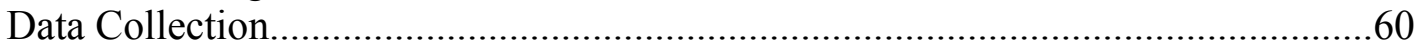

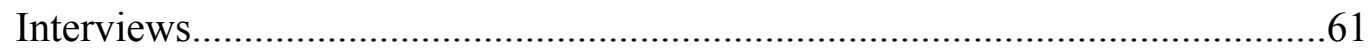

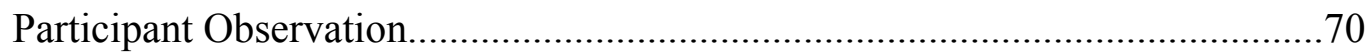

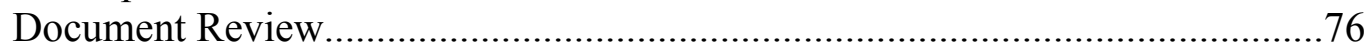

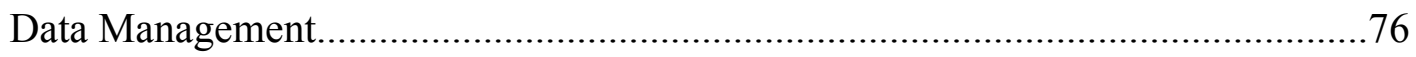

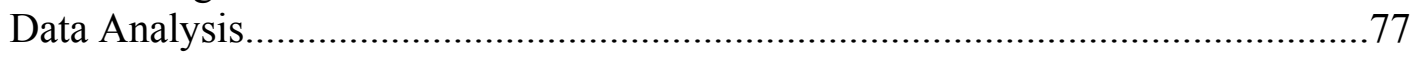

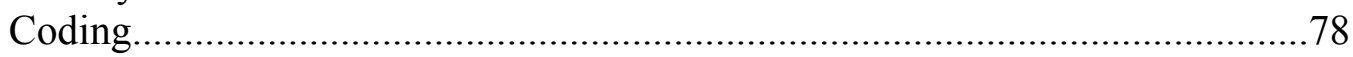

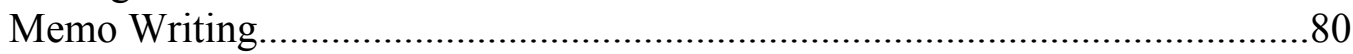

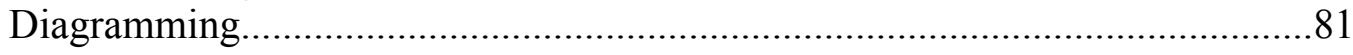

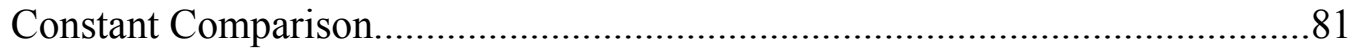

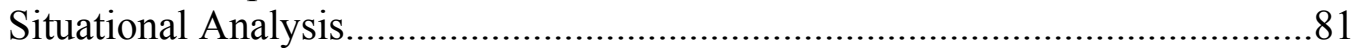

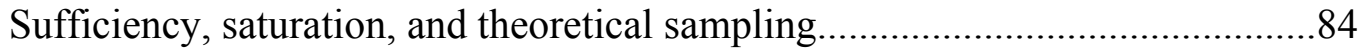

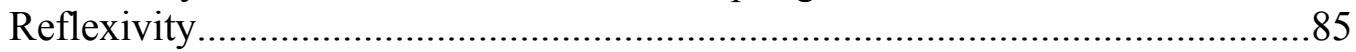

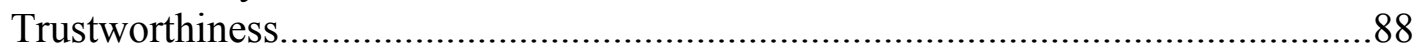

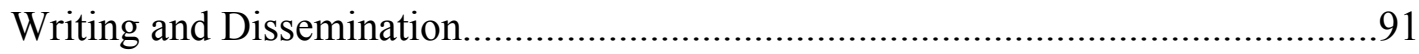

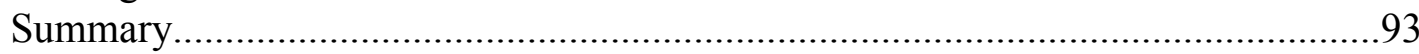




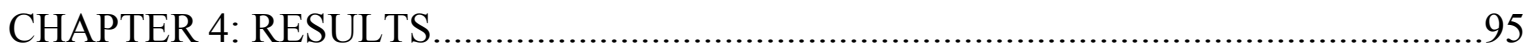

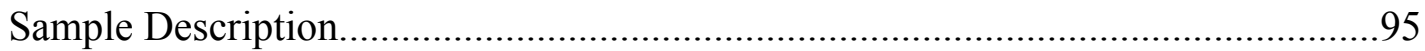

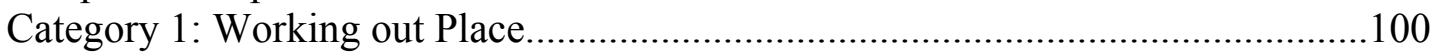

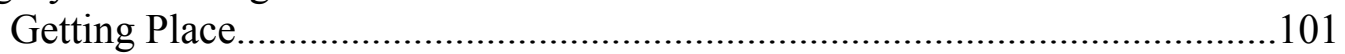

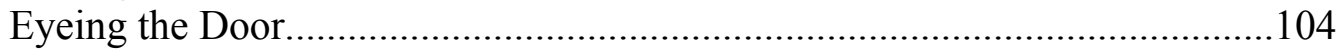

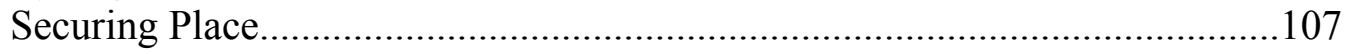

What's Five Years in a Lifetime? Working out Place with Tess.....................110

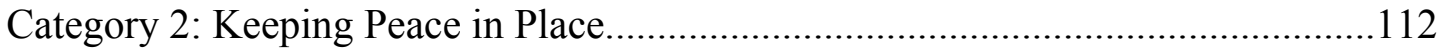

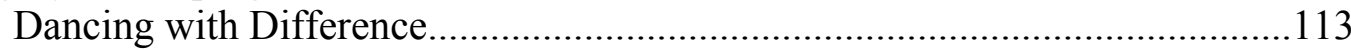

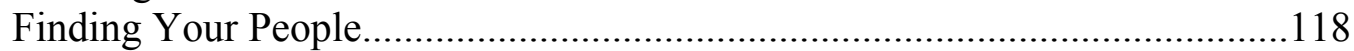

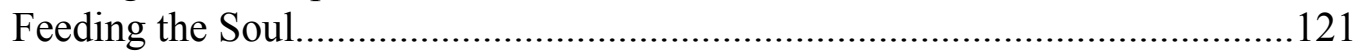

The Siren Song of the Mountain: Keeping Peace in Place with Daniel............124

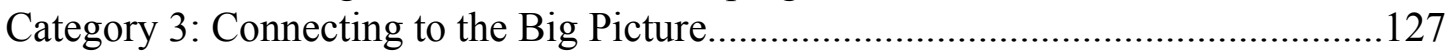

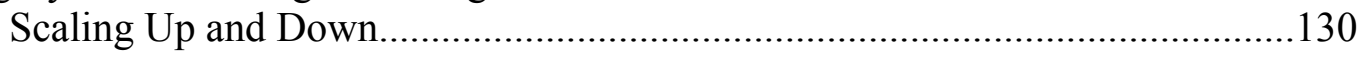

Reflecting Across Time..............................................................................134

I'm Going to Stay Here and Fight: Connecting to the Big Picture with Cat.....137

Doing Place: A Context Specific Interpretive Framework........................................139

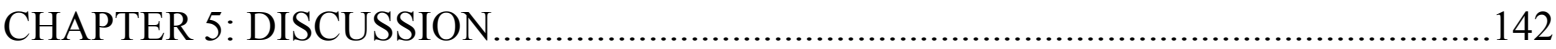

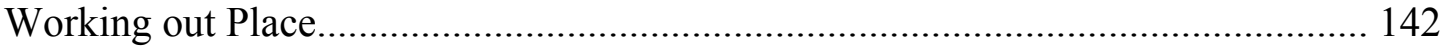

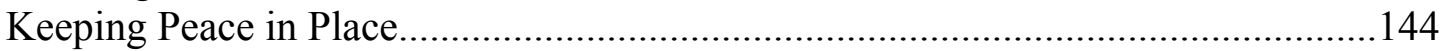

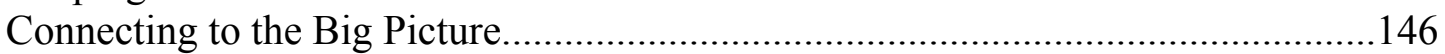

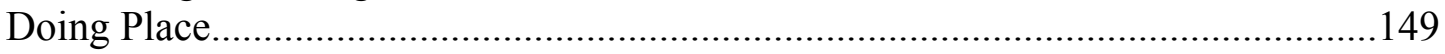

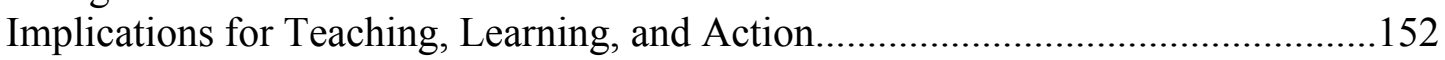

Strengths, Limitations, and Suggestions for Future Research.................................161

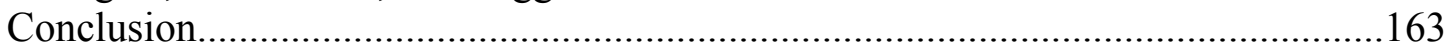

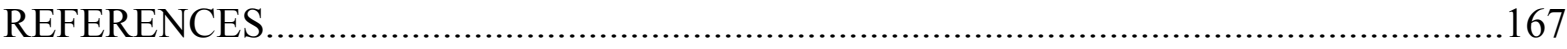

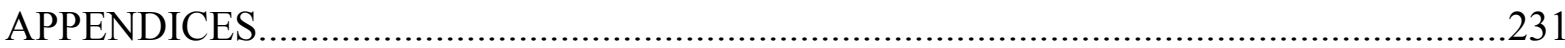

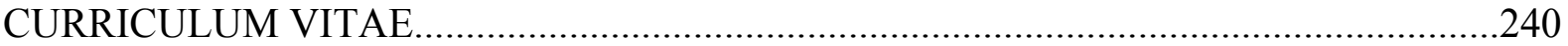




\section{LIST OF FIGURES}

FIGURE PAGE

1. Connecting to the Big Picture: A Positional Map..........................................83

2. Participants by County of Residence............................................................99

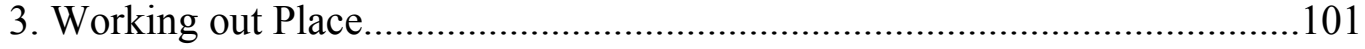

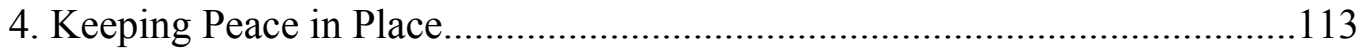

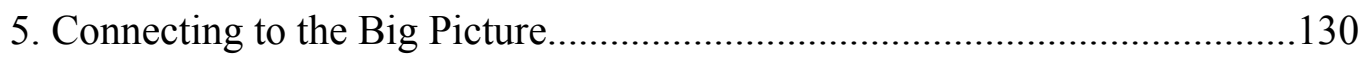

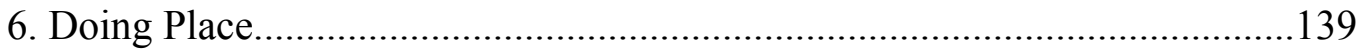




\section{CHAPTER ONE: INTRODUCTION}

I say to the young people, don't get into this with the idea that you're going to save [the world] and solve all the problems even in your lifetime. The important thing to do is to learn all you can about where you are. If you're going to work there, it becomes even more important to learn everything you can about that place, to make common cause with that place, and then resigning yourself, becoming patient enough to work with it over a long time.

-Wendell Berry

In 2013, Kentucky's beloved Poet Laureate Wendell Berry sat down in an interview with acclaimed journalist Bill Moyers. Together, they reflected on the numerous social, economic, and environmental issues faced by the global community. Contemplating a way forward, Berry (2013) reasoned it is largely young people who will inherit both the uncertainty of these conditions and the charge to respond. He also insisted that young people have the power to make transformative change by making peace with the places they call home and executing patience toward their improvement (Berry, 2013).

Such commitments are critical to the vitality of all communities, where young adults contribute new knowledge, skills, and ideas to the growth of local economies and social life (Carr \& Kefalas, 2009; Liu, 2013). Yet, high rates of outmigration have long challenged this goal in rural areas (Carr \& Kefalas, 2009; Jacquet, Gurhrie, \& Jackson, 2017; Pollard \& Jacobsen, 2018; Prince, 2014). Global trends in rural population loss are expected to continue well into the future. In fact, the United Nations (2014) projected that by 2050, 66 percent of the world and 90 percent of the U.S. population will live in cities. 
Rural outmigration is driven by a complex intersection of structural forces, local conditions, and individual and household decision-making (Black et al., 2011; Castles, de Haas, \& Miller, 2014). Among these, a number of development factors impact whether individuals and families are willing and able to stay in rural communities (Carr \& Kefalas, 2009; Castles et al., 2014; Jacquet, et al.,2017; Stuart, 2014). These include transportation obstacles, environmental threats, stifled diversity, political division, fewer job opportunities, and limited access to education, health and social services (Carr \& Kefalas, 2009; Ginsberg, 2011; Humble, Lewis, Scott, \& Herzog,2013; Jacquet et al., 2017; Lewis, Scott, \& Calfee, 2013; Stuart, 2014; Weinbaumm, 2004; Williams, 2002).

While these factors may act to "push" some people away from rural communities, there are also factors which "pull" them toward urban development (Castles et al., 2014). These include access to expanded educational and employment opportunities, the presence of a creative class, inclusive social climates, and diverse lifestyle amenities (Betz \& Partridge, 2013; Brown, 2002; Florida, 2002; McGranahan $\&$ Beale, 2002). The phenomenon of youth outmigration is often referred to as 'brain drain,' due to the fact that many young people leave in search of education and often have difficulty finding the desire or opportunity to pursue their careers back home (Artz, 2003; Carr \& Kefalas, 2009; Heinemann \& Hadler, 2015).

In addition to these material conditions, it is also important to consider the cultural meanings of migration and its role within individual life course decisions among young adults (Gabriel, 2006). American cultural narratives, for example, place great value on mobility as a symbol of social and individual progress (Toth, 2014). These sentiments may be reinforced by local educational systems which often invest the most in those who are likely to leave, or by adults with mentoring capacity who 
encourage those with the most potential to go (Carr \& Kefalas, 2009). Through these discourses and interactions, rural places are often reified as challenges to overcome or outgrow (Donaldson, 1984; McClay, 2014). Thus, many young adults come to associate staying with individually or culturally inferior identities (Easthope \& Gabriel, 2008; Wolfe, Black, Munz, \& Okamoto, 2017). It is often accepted, and even expected that young adults will leave (Easthope \& Gabriel, 2008). In these ways migration can be both depicted and experienced as a marker of personal development (Dalsgaard Pedersen \& Gram, 2017).

The consequences of young adult outmigration include changes to culture, dispersed families, demographic shifts, and economic effects including a persistently diminishing tax base, and fewer consumers, entrepreneurs, and professionals to address local challenges (Artz, 2003; Carr \& Kefalas, 2009; Heinemann \& Hadler, 2015). Given these realities, rural communities are interested in strategies that support the retention and return of young adults (Carr \& Kefalas, 2009; Wolfe et al., 2017). Critical to this pursuit is understanding why some young people choose to stay; particularly those with the motivation and skills to lend toward positive change. Yet, scholarly and practical emphasis has been upon those who leave and the resulting conditions of sending communities (Heinemann \& Hadler, 2015; Liu, 2013; Wolfe et al., 2017). In light of these concerns, it is timely to explore the experiences of young adults who make conscious commitments to improving rural communities, despite many obstacles and pressures to leave.

\section{Study Overview}

This study aimed at such an understanding by focusing on college educated young adults living in Appalachian Kentucky and working on social, environmental, and economic issues therein. The Appalachian context is appropriate for such an 
inquiry based on the largely rural character of the region and its long history of outmigration (Salstrom, 1994). A significant body of literature documents the drivers of outmigration from earlier periods, the experiences of migrants, and the struggles faced by those who stayed (e.g. Alexander, 2006; Berry, 2000; Borman \& Obermiller, 1994; Brown, 1988; Feather, 1998; Gregory, 2005; Obermiller, Wagner, \& Tucker, 2000; Philliber, 1981; Philliber \& McCoy, 1981; Schwarzweller, Brown, \& Mangalam, 1979). Today, updated demographic profiles suggest that outmigration persists in Appalachia (e.g. Betz \& Partridge, 2013; Pollard \& Jacobsen, 2018), but gaps remain in our understanding of the young adults faced with this decision, particularly those who resist migration (Heinemann \& Hadler, 2015).

Heinemann and Hadler (2015) provide a critical exception to this gap through their qualitative study of motivations to stay among twenty Appalachians in West Virginia all of whom had earned a bachelor's degree or higher. Common motivations included noted strengths of rural communities such as closeness of family, emotional attachments to community, and place based identities (Heinemann \& Hadler, 2015). However, Heinemann and Hadler's (2015) study is admittedly complicated by the diversity of participants they chose. This included participants from a wide range of incomes, ages, and careers, and with varied histories in the region (Heinemann \& Hadler, 2015). As a result, the authors (Heinemann \& Hadler, 2015) conclude that narrowing inclusion criteria in future studies would aid in-depth understanding of particular groups of 'stayers' within the region. This study attends to this need by recruiting young adults based on five narrowed criteria, including that participants: (1) currently lived in Appalachian Kentucky; (2) had lived in the region least 15 years (cumulatively); (3) were Millennials born between the years 1980 and 2000 (see 
Vespa, 2016); (4) had completed or attained some college education; and (5) were working on social, economic, and environmental issues within the region.

The present inquiry also extends Heinemann \& Hadler's (2015) work by moving beyond motivations to stay and exploring the experience and meanings associated with this decision. This approach draws upon the aforementioned cultural approaches to migration and evidence that staying in rural areas presents a number of personal challenges for those who engage in local change efforts. For example, research in Appalachia demonstrates that working on social, economic, and environmental issues is a highly political endeavor that can strain both personal and professional life in the region (e.g. Bell, 2013; Hatch, 2009). In a qualitative study with young people in rural Ohio, Wolfe and colleagues (2017) found that participating in local democratic processes required both management of personal identities and negotiation of the stigmas associated with staying in the region.

Drawing on these insights, two primary aims were established for this study: 1) Understand and provide deep description of young adults' experience of committing to stay and work in Appalachian Kentucky and the meaning they make of these decisions and 2) Develop a context specific framework for interpretation of these decisions and processes as grounded in participant experience. Questions supporting and guiding these aims included: 1) What processes inform young adults' decisions to live and work in Appalachian Kentucky? 2) What strategies do young adults engage to maintain these commitments? And 3) How do young adults make meaning of these commitments? The nature of these questions warranted a qualitative approach designed to gain in depth understanding from individuals who have directly encountered these decisions and processes (Padgett, 2008). 
The remainder of this introduction provides additional rationale for these aims and criteria. Section One addresses the study's approach to Appalachia and articulates the regional definition used to recruit participants. This is followed in Section Two by a brief history of the region including an emphasis upon social, environmental, and economic issues and their intersection with migration trends. Section Three provides an overview of present economic, environmental, and social challenges and highlights efforts to shape a new future for the region. Section Four focuses on the importance of young people's contributions to regional solutions and further explains the inclusion criteria. As this study is being conducted in pursuit of a doctoral degree in social work, Section Five illustrates the connections between the aims of this study and the profession's goals and interests. This introduction concludes with an outline of subsequent chapters.

\section{Approaching Appalachia}

In his seminal essay on Appalachia in the American consciousness, Henry Shapiro (1977, p. 43) reflected that images and descriptions of the region often depict it as "an anomaly in an otherwise unified and homogenous nation" as somewhere "in but not of America." Through these reflections, the region has often served as a symbolic gauge for the success of the country at large (Shapiro, 1977). This has been true since the late $19^{\text {th }}$ Century, when "local color" writers visited Appalachia and described the lack of "progress" they observed (Batteau \& Obermiller, 1983).

Early among these was Harney’s (1873) essay, ‘A Strange Land and Peculiar People,' which depicted mountain communities as 'frozen in time, unaffected by modernization" (Haynes, 1997, p.47). To outsiders like Harney, the rugged geography and local culture represented both physical and social barriers to growth and development (Eller, 2001; Shapiro, 1977; Williams, 2002). By framing it as in need of 
outside assistance, such works aided in the justifications of many missionaries and other social reformers who arrived to "save" the region (Eller, 2001; 2008; Fraley, 2011; Haynes, 1997; Shapiro, 1978).

Since that time, countless writers, filmmakers, scholars, journalists, activists, and politicians have used Appalachia to both critique and support broader social issues (Eller, 2008). Recent examples include the seemingly endless series of journalistic accounts that attempt to explain the social, economic, and political character of "Trump Country" following the 2016 Presidential election (e.g., Associated Press, 2017; Kaplan, 2016; MacFarquhar, 2016; see Catte, 2018). Others continue to exploit and perpetuate well-worn cultural stereotypes (Billings, Norman, \& Ledford, 1999; Catte, 2018), including through television programs like The Discovery Channel's Moonshiners docudrama (Magilla Entertainment, 2011-), and MTV's so called "reality” show Buckwild (Poznick, et al., 2013-). In literature, J.D. Vance's (2016) Hillbilly Elegy has been widely critiqued along these lines (e.g. Catte, 2018; Harkins \& McCarroll, 2019).

Such accounts render Appalachians as, at best, passive or naïve victims; ignoring their long history of resistance and innovation and simplifying the root of problems outsiders are so quick to identify (Catte, 2018; Fisher, 1993; Weinbaumm, 2004; Williams, 2002). Acknowledging this history, issues of representation are of paramount concern in any scholarly endeavor conducted in and about the region (Billings et al., 1999). As an Appalachian myself, I approach this issue with a deep sense of responsibility to get it "less wrong" (Engelhardt, 2017, p.4). By focusing on young adults who are actively working to improve their communities, great care was taken to honor their ability to define themselves, to create solutions for local and 
regional challenges, and to articulate the meaning of their experiences through the course of this research.

Still, a working definition of the region was needed for the purpose of contextualizing the research environment and recruiting participants. This is no simple task given Appalachia has a long and complicated past when it comes to definitions (Fisher \& Smith, 2012). The first known appearance of the word “Apalachen" is from a European map dated 1562 (Williams, 2002). The label is storied to have originated by way of the Apalachee people of Northern Florida, who redirected Spanish explorers to a distant mountain range in their search for gold (Williams, 2002). The mountains became collectively called the "Appalachians" following the American Revolution (Williams, 2002).

Today, geography continues to encompass the most commonly referenced set of boundaries for the region; those established by the Appalachian Regional Commission (ARC) in 1965. The ARC includes parts of 12 states and all of West Virginia in its definition, an area that spans 205,000 square feet following the ridge of the Appalachian Mountains from New York to Mississippi, and is home to over 25 million people (ARC, 2009; Marshall et al., 2017). This study adopts the ARC's definition for the purpose of recruiting residents of Appalachian Kentucky, which includes 54 contiguous counties in the eastern part of the state.

All of Kentucky's Appalachian counties fit within the ARC's sub-region of Central Appalachia (ARC, 2009). The U.S. Department of Agriculture's (2017) Rural-Urban continuum codes designate 26 of these counties as entirely rural, 24 as non-metro, and the remaining 4 as metro counties with populations of less than 1 million. However, all but three of Kentucky's Appalachian counties are considered rural for the purposes of federal programs and initiatives, such as those using the U.S. 
Office of Rural Health Policy (2016) classifications. Though the ARC definition facilitates clarity in this study and serves operational mandates for agencies and programs, it is important to note that the ARC's boundaries have long been contested (Williams, 2002). For example, parts of Virginia were excluded from this definition as a results of one congressman's philosophical objection to the entire ARC project (Williams, 2002).

Moreover, any study of the region must also contend with other attempts to define the region including those that draw upon sociocultural, political, and even cognitive dimensions (Ergood \& Kuhre, 1991; Fisher \& Smith, 2012). However, these aspects of Appalachian identity are also nuanced and strained (Batteau \& Obermiller, 1983). Reactions to media, artistic, and scholarly representations, for example, have generated reluctance to define or portray a united Appalachian identity or culture (Keefe, 1998, 2005). Scholars have even argued that the earliest depictions of Appalachian communities effectively invented the idea of a distinct regional culture (e.g., Batteau, 1990; Eller, 2008; Precourt, 1983). At the same time, uniqueness has been documented in what is described as a core culture found predominantly in Central Appalachia and its periphery (Campbell, 1921; Keefe 2005). Keefe's (2005) review of this literature identifies common Appalachian characteristics such as distinct dialects (e.g. Puckett, 2000; Williams, 1972; Wolfram \& Christian, 1976); kinship and family based social structures (e.g. Batteau 1982a, 1982b; Beaver, 1986; Bryant, 1981; Halperin, 1990; Keefe, 1998; Matthews, 1966), the importance of community to both sense of identity and as a source of structure (e.g. Brown 1988; Hicks, 1976; Kaplan, 1971; Pearsall, 1959; Stephenson, 1968), the strong influence of religious heritage and institutions (e.g. Dorgan, 1987, 1989; Humphrey, 1984; McCauley, 1995; Sovine, 1983), and an overall rootedness in place (e.g. Allen, 1990; 
Foster, 1988; Humphrey, 1984). These accounts also note that Appalachian values typically include familism, individualism, religious world views, emotional connections to land, and camaraderie among neighbors (e.g. Beaver, 1986; Jones, 1994; Keefe, 1998).

While these cultural elements have certainly shaped the region's history and young people's experiences therein, this study recognizes that, "[1]ike other American minority groups, Appalachians resist classification. Their culture is dynamic and they are constantly in the process of re-defining their identity" (Eller, 2001, p. xi). Thus, no attempt is made here to locate a singular cultural identity that defines Appalachia, and it is accepted that many exceptions to and transformations of these patterns exist (Keefe, 2005; Lewis, 2001). What is more, this study positions the negotiation of regional identity as a potentially critical process that may inform young adults' decisions to stay, as well as their desires for transformative change (Towers, 2007; Wolf et al., 2017)

\section{Understanding the Past}

Although the purpose of this inquiry is not to generalize findings toward the entire Appalachian region, it is important to situate young people's experiences within their broader context. While others offer more comprehensive histories (e.g. Williams, 2002; Eller, 2008), what is offered below is a necessarily condensed overview. This includes a selection of social, environmental, and economic issues and their intersections with migration trends over time. These are shared as relevant background to understanding present day circumstances faced by young people and the Appalachian communities where they live and work.

Upon entering Appalachia, colonizers and settlers met diverse peoples including Mississippian, Cherokee, and Creek (Williams, 2002). Europeans occupied 
the region in the eighteenth and nineteenth centuries, largely at the expense of these groups (Boyd, Jr., 2004; Williams, 2002). Pioneers and settlers lived predominantly subsistence lifestyles based on farming, foraging, hunting, and fishing (Davis, 2000; Eller, 2008; Keefe, 2005). Prior to the industrial age, Appalachian settlements were relatively isolated and engaged primarily with their own internal concerns (Eller, 1982; Lewis, 2004).

In the early nineteenth century, the region provided raw materials like timber and coal to fuel industrialization and development elsewhere in the country (Batteau \& Obermiller, 1983; Eller, 2008). During this time, the introduction of railroads and the building of towns and roads forever altered life in the region (Eller, 1982). Among the most significant changes was through the decrease in farm productivity and income, and transition into industrial and service sector employment including mining, logging, and work in textile mills (Eller, 1982). Between 1830 and 1860, the region's population grew more than four times its pre-industrial size (Moore, 1991; Williams, 2002). This rapid growth and modernization changed the ways in which Appalachian communities conducted politics, and civic engagement witnessed decline as the region became more and more controlled by industrialists and absentee land owners (Eller, 1982).

During the Civil War, the region was known for strong Union solidarity and less dependency upon the slave economy compared to states further south; a reputation which allowed for modest improvements in racial integration in some communities (Inscoe, 2004; Williams, 2002). Following the war, the population continued to grow in size and diversity, but resources and natural landscapes were depleted and the culture was overwhelmed by hostility and hardship (Eller, 2008; Lewis, 2004; Williams, 2002). The regional struggles that followed were exported as 
attributes of the Appalachian people through caricatures of the rugged mountaineer and hillbilly, tales of feuding families, and other exaggerated images (Williams, 2002). Regional race riots and severe discrimination against African Americans also left a lasting legacy (Griggs, 2002).

As a result of these many challenges, the value of the region's natural resources was often regarded higher than its people and their connections to place. This is evidenced by multiple proposals for sponsored depopulation in the region. Such strategies were promoted in 1911 to support the region's use as a timber economy and again for national parks in the 1920s (Eller, 2008; Williams, 2002). During the Great Depression, when more than half of the region received some form of public assistance, planned outmigration was again proposed to facilitate the building of dams (Eller, 2008; Williams, 2002).

Through Roosevelt's New Deal initiatives, the Wagner Act of 1935 legitimized union organizing and sparked a new wave of resistance among mining families and communities (Lewis, 2004). The establishment of the Tennessee Valley Authority aimed to modernize the region and spark economic growth aided by higher electric power and the ongoing recruitment of industry (Lewis, 2004; Salstrom, 2004). Large regional systems and interventions such as national parks, small scale manufacturing cooperatives, craft revivals, and other public works also changed landscapes and opportunities (Salstrom, 2004; Williams, 2002). Federal changes to agricultural policies during this period disproportionately benefited larger operations and Appalachian family farms continued to decline (Salstrom, 2004).

At the onset of World War II, coal and timber industries boomed once again through supplying myriad war efforts, and Appalachian labor was mobilized for jobs in defense industries and military positions in urban centers (Brown\& Hillery Jr.; 
Eller, 2008). While outmigration was not an entirely new phenomenon, this wartime effect brought about new hope among young people who had already survived many hard times (Eller, 2008; Williams, 2002). Between 1940 and 1942 alone, over 85,000 people left Appalachian Kentucky; as much as 40 percent of the region's young men (Larson, 1943; Eller, 2008).

After the war, Appalachia's economic and political landscape reflected the post-industrial world full of changing forces at national and international levels (Batteau \& Obermiller, 1983). Returning from wartime activities, younger Appalachians were met with a decreased demand for their labor, partly as a result of the ongoing mechanization of mining activities, but also because the nation was slowly shifting to oil and natural gas resources (Eller, 2008). During this time, millions of Appalachians joined workers from further south in the Great Migration to industrial cities outside the region; with higher rates among the region's African American residents (Eller, 2008; Williams, 2002). Young adults moved in search of work and new opportunities, as well as to find better education, housing, and healthcare for their families (Eller, 2008). It was also difficult to attract young Appalachians home, as their lifestyles and expectations had changed during their time away (Drake, 2001; Eller, 2008). Total regional population loss from the 1940s is estimated at approximately 250,000 residents (Brown \& Hillary, Jr., 1962; Eller, 2008).

In the 1950s, Appalachian Kentucky lost another 35 percent of its population (Brown \& Hillary, Jr. 1962; Eller, 2008). Influences during this decade included unintended effects of labor negotiations between the United Mine Workers Association and coal producers (Eller, 2008). While initial agreements improved wages and benefits for miners, they also removed their right to strike and privileged 
mechanized mining operations (Eller, 2008). Subsequent negotiations effectively wiped out small mining operations and displaced many workers over the next thirty years (Eller, 2008; Lewis, 2004). A growing number of Appalachian people came to rely upon federal assistance, and the poor were increasingly blamed for their struggles (Eller, 2008). The region also witnessed devastating effects of widespread flooding in 1957, which resulted in part from decades of strip mining and logging (U.S. Forest Service, n.d.; Eller, 2008). Near the close of the decade, politicians and researchers became interested in identifying and explaining the region's many shortcomings (Drake, 2001). While old strategies like government sponsored outmigration were again on the table (e.g., Luebke \& Hart, 1958; see Eller, 2008; Williams, 2002), these conversations sparked a new era of development initiatives and interventions (Drake, 2001).

By the 1960s, bust conditions had returned as a result of diminishing coal prices and the continued displacement of mine workers (Eller, 2008). Outmigration continued and images of those who remained shocked the nation through numerous journalistic and fictional accounts, including those that followed President Kennedy on the campaign trail (e.g. Harrington, 1962; Dominis, 1964; see Drake, 2001; Eller, 2008). The region became a laboratory for experimentation among governmental programs and scientists interested in developing tools for the "War on Poverty," declared by President Johnson from the front porch of a home in Martin County, Kentucky (Eller, 2008).

President Johnson carried out this project through two primary policy dimensions in the region. First was the Economic Opportunity Act of 1964, which initiated a number of programs that continue to serve Appalachian Kentucky today including Head Start, AmeriCorps VISTA (Volunteers in Service to America), and 
the Upward Bound and Community Action Programs (Drake, 2001). The second dimension came in 1965, through the establishment of the ARC as a partnership between state and federal administrations which would oversee the growth and development of Appalachian counties (Drake, 2001; Eller, 2008). Combined with the privately funded "Appalachian Volunteers" program, these initiatives recruited many educated young adults to serve the region (Drake, 2001). Impacted by larger social movements and the presence of these young adults, Appalachia became a "hot bed of social action" during this time (Eller, 2004, p. 210). Included among these efforts for example, was the work of Appalshop in Whitesburg, Kentucky; an organization which has used media and arts based strategies to diversify regional storytelling and facilitate local change since its establishment in 1969 (Appalshop, 2018).

These social movements also impacted a renewed interest in rural life, positioning Appalachia as an ideal destination for young people returning "back to the land" through the 1970s (Salstrom, 2004). Simultaneously, this decade saw a rise in scholarly interest in Appalachian studies and the creation of many regional academic programs (Eller, 2008). However, this period also witnessed a number of events and circumstances which continued to impact development and migration trends. These included a national energy crisis, renewed struggles for mine workers' unionization, black lung advocacy and legislation, the introduction of mountaintop removal mining, and another series of devastating floods (Eller, 2008; Williams, 2002).

Throughout this history, coal producing communities have been particularly hit hard by decades of boom and bust cycles in production and subsequent population loss (Kratzer, 2015; Obermiller \& Howe, 2000; Pollard, 2005; Stone, 2016). In total, more than 3 million people left Appalachia for employment, education, and other opportunities between 1940 and 1970; many of them young adults (Eller, 2008; see 
also Berry, 2000). The routes taken by these migrants were informed by extensive networks along family and community lines and large populations of urban Appalachians were seen in neighborhoods across northern cities like Cleveland and Detroit (Eller, 2008). Migrants often worked and lived in poor conditions and faced discrimination based on exported stereotypes (Eller, 2008). Still, many others found the amenities of urban life advantageous. Women in particular became accustomed to the benefits of modern conveniences and the opportunity for their own employment and education outside the home (Eller, 2008).

Subsequent decades are characterized by increasing economic gaps between Appalachia and the nation (Eller, 2004). Although some improvements were made in the most distressed counties, disparities within the region also persisted (Eller, 2004, 2008). The most significant advances occurred along highway corridors with easier access to diverse economic markets and millions of dollars were invested in roads and transportation in this era (Franklin, 1981; see also Eller, 2008). Outmigration also continued (Eller, 2004), but so did the efforts of local activists and community organizers through new grassroots organizations like Kentuckians for the Commonwealth (KFTC) and the Community Farm Alliance, which grew out of the 1980s and are still active today (see Fisher, 1993).

While government and philanthropic initiatives changed the appearance of Appalachian communities over time, these developments did not alter other fundamental issues and in some cases created new ones (Eller, 2008). These include political corruption, lack of long term economic sustainability, environmental exploitation, and the degradation of communities and culture that persisted well into the 1990s and early 2000s (Drake, 2001; Eller, 2008). Industrial recruitment continued to be a prominent economic development strategy in the region, such as 
through the federal designation of Jackson County, Kentucky as a Clinton

Empowerment Zone (Collins \& Dewees, 1999; Weinbaumm, 2004). This period also saw a rapid increase in diversity (Pollard, 2003) alongside resentment toward racial and ethnic minorities who were often blamed for cultural and economic shifts in an era of globalization and change (Billings, 1999).

\section{Contextualizing the Present}

Today, there are no formal proposals to move Appalachians in mass. However, it is still common for accounts of the region's problems to suggest the obvious solution for the region's residents is to simply move (e.g., Lowrey, 2014; see Catte, 2018). Indeed, many continue to do so. Adults between the ages of 18 and 24 currently make up less than 7.5 percent of the population in much of rural Appalachia, where the national average is 9.5 percent (Pollard \& Jacobsen, 2018). This age group falls below the national average in all but five of Kentucky's 54 Appalachian counties (Pollard \& Jacobsen, 2018). Combined with the long history of outmigration described above, these figures illustrate the continued significance of young adults' commitments to staying and working in the region.

Though the emphasis in this study is on the active strategies and processes young adults enact toward positive change, it is important to illustrate the magnitude and scope of the challenges they are attempting to address. Thus, the discussion now turns to some of the dominant economic, environmental, and social issues in the region. Though not exhaustive, the issues selected for this review were chosen based on their significance to sustainable development and well-being in the region (House, 2014; Sachs, 2015; Schumann, 2015), their function as migration drivers (Black et al., 2011; Liu, 2013; McLaughlin et al., 2014; Shumway, Otterstrom \& Glavac, 2014) and their importance to the professional aims of social work, to which this study 
subscribes (Slovak, Sparks, \& Hall, 2011). This includes persistent poverty and issues of economic development, environmental concerns regarding mining and other regional activities, and prevailing social concerns including health disparities, education, and issues of diversity and representation. The discussion of challenges is followed by a description of select strategies that are currently being implemented in the region to address these issues.

\section{Economic Issues}

As noted above, Appalachia's poverty rate has been one of the most consistent markers of the region's progress over time (ARC, 2015a). Despite the best efforts of the so called "poverty warriors" and policy makers of previous eras, Appalachian poverty and its associated stereotypes have not been ameliorated (Eller, 2008). Between 2012 and 2016, all but one of Kentucky's Appalachian counties had poverty rates above the U.S. national average of 15.1 percent (ARC, 2018b). Forty-two counties saw poverty rates beyond 150 percent of the national average during this time (ARC, 2018b). In the same period, poverty rates increased among all ages under 65 in Appalachian Kentucky compared to the previous five years (Pollard \& Jacobsen, 2018).

Presently, the ARC (2018a) designates 37 of Kentucky's 54 Appalachian counties as economically distressed; ranking them in the bottom 10 percent of counties nationally. Among the factors impacting these figures include a persistent lack of economic diversification and dependence on extractive industries in the region. Between 2005 and 2015, coal production in Appalachia dropped by 45 percent compared to 21 percent nationally (Bowen, Christiadi, Deskins, \& Lego, 2018). As coal and other industries have declined over the years, new social infrastructures have taken the place as the largest employers in many communities; namely schools, 
hospitals, and other public services (McGreal, 2016; Quinton, 2016). Still, between 2012 and 2016, unemployment rose in many Appalachian Kentucky counties; reaching 8.3 percent in the sub-region overall (Pollard \& Jacobsen, 2018). Seventeen counties in Appalachian Kentucky have an unemployment rate of 10 percent or higher and many have labor force participation rates of less than 60 percent (Pollard \& Jacobsen, 2018). These too are well above the rates in non-Appalachian Kentucky and nationally (Pollard \& Jacobsen, 2018).

\section{Environmental Issues}

As Appalachia's economy has been highly reliant upon natural resource extraction, environmental issues have also long plagued the region. The environmental impacts of coal mining include irreversible changes to landscapes, damages to wildlife ecosystems, degradation of soil, air, and water quality; as well as impacts through generation of greenhouse gases (Morrice \& Colagiuri, 2013). These have significant effect in quality of life in the region (Morrice \& Colagiuri, 2013). For example, contamination of water impacts gardening and farming, recreational opportunities, cooking and cleaning, and requires many residents to invest in filters and bottled water (Blakeney \& Marshall, 2009; Fraley, 2007; McSpirit \& Reid, 2011). It may also cause delays or restrictions in access to a number of public facilities like schools (Blakeney \& Marshall, 2009).

While the region is best known for its coal production, there are many other areas of environmental concern. Significant among these include the targeting of the region's rich shale formations by the hydraulic fracturing, or "fracking" industry (Morrone, Chadwick, \& Krise, 2015; Waples, 2012). This practice includes the potential for additional water contamination, earthquake risk, negative public health outcomes, and the possibility that the economic and social effects will burden 
communities already exposed to the insecurity of coal production cycles (Morrone et al., 2015; see also Brasier, et al., 2013; Clark, Brunham, Harto, \& Horner, 2012; Kim, 2013; Stedman et al., 2012; Vidic, Brantley, Vandenbossche, Yoxtheimer, \& Abad, 2013; White, 2014). There is also increasing unease over proposals to convert aging natural gas pipelines to carry the natural gas liquids (Brammer, 2018) and an ongoing legislative and cultural battle over renewable energy sources (Loftus,2019). Moreover, many communities face compounding environmental troubles related to local practices in conservation, agriculture, sewage and waste disposal, and water treatment. Martin County for example, has been cited nationally due to its long history of water quality issues, which lead to a declaration of a stage of emergency in 2018 (Frost, 2018; Hinckley, 2017).

\section{Health Disparities}

Environmental issues in Appalachian Kentucky also exacerbate health disparities. Well-known, for example, are the occupational effects of coal-mining, including black lung disease; rates of which have recently hit a 25-year high (Blackley, Halldin, \& Laney, 2018; Estep, 2018). Evidence is also building to suggest that merely living near coal mining activities places Appalachian families at higher risk of health issues including: lower birth weight (Ahern, Mullett, MacKay \& Hamilton, 2010), cardiovascular disease, angina and heart attacks (Hendryx \& Zullig, 2009), COPD and hypertension (Hendryx \& Ahern, 2008), and cancer (Hendryx, Wolfe, Luo \& Webb, 2011). Residents in areas with mountaintop removal mining additionally experience elevated risk of birth defects and poorer self-rated physical and mental health (Ahern et al., 2011; Hendryx \& Innes-Wimsatt, 2013; Zullig \& Hendryx, 2011). 
But these are not the only health concerns pervasive in the region. Comparing Appalachian counties with non-Appalachian counties across the country, Marshall and colleagues (2017) found that the region underperforms on 33 of 41 health indicators examined. These include leading causes of death (e.g., heart disease, cancer, stroke), lifestyle factors (e.g., smoking, lack of physical activity), physically and mentally unhealthy days, obesity, and availability of healthcare professionals (Marshall et al., 2017). Appalachia is also witnessing a more rapid increase in all cause mortality rates (Meit, Hefferman, Tanenbaum, \& Hoffman, 2017). Among adults aged 25 to 34, mortality rates were 55 percent higher among Appalachians as compared to non-Appalachians (Meit et al., 2017).

Over the last decade, attention has also turned toward "diseases of despair" within the region, which include those related to alcohol and drug use, as well as suicide (Case \& Deaton, 2015; Meit et al., 2017). Combining mortality from all of these, Meit and colleagues (2017) found that rates in Appalachia were 37 percent higher than those in non-Appalachian parts of the country. These disparities were stark among adults aged 25 to 44 years old; where rates were more than 70 percent higher among Appalachians (Meit et al., 2017). Within Kentucky, Appalachian counties have a 26 percent higher rate of mortality from diseases of despair than nonAppalachian counties (Meit et al., 2017).

In addition to noting the economic, social, and political inequalities that contribute to health risks (e.g. Borak, Salipante-Zaidel, Slade, \& Fields, 2012; Kozlowski \& Perkins, 2016), Appalachian fatalism has been suggested as informing the region's persistent disparities (e.g. Hendryx, 2009; Welch, 2011). However, others note fatalism may not play as large a role in regional health decision making as has been assumed (Coyne, Demian-Popescu \& Friend, 2006). Where it is present, it may 
act as a culturally appropriate framework of explanation, rather than a driver of delayed medical attention or other behaviors (Drew \& Schoenberg, 2011).

\section{Educational Parity}

The Appalachian region also continues to lag behind the rest of the country in terms of educational parity (ARC; 2015a; Marshall et al., 2017). Between 2012 and 2016, 23.8 percent of Appalachian Kentuckians over 25 had less than a high school diploma (Pollard \& Jacobsen, 2018). In the same period, only 56.1 percent of Appalachian Kentuckians over 25 had earned a high school diploma, 6.7 percent an associate degree, and 14.3 percent a bachelor's degree or more (Pollard \& Jacobsen, 2018). All of these percentages were lower than those of non-Appalachian Kentucky and the United States in the same period (Pollard \& Jacobsen, 2018). Despite their comparatively low rates, it is important to recognize that these numbers represent improvements from previous periods (Marshall et al., 2017; Pollard \& Jacobsen, 2018).

\section{Diversity \& Representation}

While the Appalachian region has grown in diversity since 2010, still, only 1.9 percent of Appalachian Kentucky's population are non-Hispanic Blacks, 1.6 percent are Hispanic or Latino, and 1.8 percent are declared Other races or ethnicities (Pollard \& Jacobsen, 2018). This is compared to 10.3 percent, 4.2 percent, and 4 percent in Non-Appalachian Kentucky, respectively and even more diversity across the country at large (Pollard \& Jacobsen, 2018). As reflected in the region's history, issues of racism and discrimination against African Americans and other minorities has been a persistent challenge in the region (e.g., Billings, 1999; Griggs, 2002); although not one that is distinctly Appalachian (House, 2014). 
Today, there continues to be concern over the prevalence of racist attitudes and discrimination within the region (Beckett, 2017; Chae et al., 2015; Osborne, 2004; Wright, 2018). as well as increasing attention to homophobia and other forms of mistreatment (Brashear, 2014; Fischer \& Smith, 2012; Gray, 2018; House, 2018). In connection, scholars and activists have pointed to the ways that various Appalachian narratives have framed the region as one devoid of cultural complexity and diversity; effectively increasing the invisibility of minorities and experiences of difference that include those of race and ethnicity, gender expression and sexuality, and also of political and religious affiliations and other identities and positions (Anglin, 2004; 2016; Eller, 2001; Fischer \& Smith, 2012; Gray, 2018; hooks, 2014; House, 2018; Lewis, 1999; Mann, 2014; Smith, 2016). There is also widespread recognition that these issues are intimately tied to both young adults' decisions and abilities to stay (Daniels, 2014; House, 2014; McMichael, 2014) and to the sustainability and progress of the region's communities (Brashear, 2014; Johnson, 2014).

\section{Rising to the Challenge}

Although economic, environmental and social issues were presented separately in this review, it is important to insist upon the intimate connections they each have with one another for individuals, families, and communities. Similarly, strategies to address these concerns often cross issues and domains. Here, a number of examples are provided as evidence of present day motivations and movements to create positive change and as a backdrop to the types of work young adults in this study are engaging and responding to in Appalachian Kentucky.

At the federal level, significant attention has been paid to economic and social disparities within the region. President Obama declared an eight county area in 
Appalachian Kentucky as the country's first rural Promise Zone, where partners concentrate efforts for investment in community and economic development (Rickett, 2017). Connecting economic and social needs, the region has also been awarded three Promise Neighborhood grants; each of which awarded Berea College \$30 million dollars for school based initiatives that reach children and families in Appalachian Kentucky in order to improve both educational and social outcomes (Partners for Education, 2017). Significant at the state level is the Shaping our Appalachian Region (SOAR) initiative, which targets economic growth through programs that reach all domains. SOAR hopes to add 30,000 jobs to Appalachian Kentucky through projects related to broadband access, local food systems, tourism, healthcare, small business, and workforce training (Estep, 2017; SOAR, 2016).

Grassroots organizations like KFTC (2018) and the Kentucky Student Environmental Foundation are also working hard for a Just Transition away from coal dependency. Joining with other organizations as well as citizen led efforts, their strategies attend to both economic and environmental concerns and include organizing protests against current destructive practices, lobbying political leadership to embrace renewable energies, providing training for displaced miners, and promoting principles of sustainability. Substantial faith-based environmental initiatives within the region promote pro-environmental behaviors and policies through an emphasis upon spirituality, morality, and stewardship (Billings \& Samson, 2012; Davis, 2004; Feldman \& Moseley, 2003; Jolly \& Jones, 2015; Rice \& Burke, 2018).

There also continues to be significant efforts to both empower and meet the needs of those living in poverty within the region (e.g., Christian Appalachian Project, 2017; Rogers, 2018) as well as to encourage and support local entrepreneurship and 
art based initiatives that contribute renewed vitality to the region's economies and social life (Mountain Association for Community Economic Development, n.d.; Stephens, Partridge, \& Faggian, 2013; Turpin, 2015; Wojan \& Nichols, 2018). In fact, the arts have also played an important role in exposing health concerns and issues of diversity and representation. For example, Harlan County's community theatre group Higher Ground developed and toured a program titled "Needlework," which drew on and sensitively explored the region's substance use challenges (Griffey, 2018). What is more, there are significant efforts to highlight bright spots in well being (Lane et al., 2018), as well as to develop culturally and geographically tailored programs and interventions that address health disparities (e.g. Schoenberberg et al., 2015; Haddox, 2018).

In addition to the progress made in each of these approaches, there are increasing efforts to reframe and reclaim narratives of membership and belonging as part of both the region's rich history and its future (Fischer \& Smith, 2012; Gray, 2018; hooks, 2014; House, 2018). The arts have been a significant driver of change in this regard, as have entrepreneurs and the larger creative class who are building inclusive spaces and places across the region (Adams, 2017; House, 2014; 2018). Issues of diversity and representation have also been addressed through the initiation of local Fairness Ordinances (Fairness Campaign, 2018; House, 2014) and the leadership of youth focused organizations which provide platforms for sharing experiences and working toward regional changes that emphasize inclusion. (e.g., Stay Together Appalachian Youth, 2018; Wright, 2018).

Whereas Appalachians have long been aware of and responsive to regional challenges, this brief preview demonstrates the new energy present in the region and the drive to imagine and create a transformative future. Combined, this momentum 
serves to inspire new generations of young people to take pride in their communities and reframes narratives that encourage them to leave (Gabriel, 2006; Toth, 2014; Carr \& Kefalas, 2009; Wolfe et al., 2017). As the next section discusses, it is imperative that young adults not only witness these changes, but that they have a voice at the table and a hand in shaping the future.

\section{Focusing on Young Adults}

It is now well established that young adult migration from Appalachia persists alongside a number social, economic, and environmental challenges. By focusing on young adults who choose to stay and work in Appalachian Kentucky, this study builds on regional efforts to recruit and retain this population in hopes that they inspire and inform new directions for the future (ARC, 2015b). This section provides rationale and definitions that support the inclusion criteria established for recruiting participants.

\section{Defining Young Adults}

Young adulthood has been defined in various ways including for legal, biological, psychological, and cultural purposes (Vespa, 2016). As a distinct developmental stage, young adulthood is often conceptualized as beginning at age 18 and lasting until the mid 20s (e.g. Simpson, 2008). Others take a broader approach, such as a recent U.S. Census study which framed young adulthood as occurring between 18 and 35 years of age; the purpose being to align with framings of the Millennial generation (Vespa, 2016). This inquiry also adopts a generational approach, but includes young adults born between the years 1980 and 2000, or between 18 and 38 years of age at the time of initial interview. Although definitions vary (e.g., Dimock, 2018; Vespa, 2016), this age range encompasses an even more expansive definition of the Millennial generation (see Stein, 2013). Representing 
roughly 80 million Americans, Millennials make up the largest generation today and attention is warranted for understanding their shared experiences and considering how they will shape the future (McDonald, 2015; Tanenhaus, 2014).

A great many characteristics have been used to label and describe Millennials. They have been dubbed the "me me me generation" (Stein, 2013) and "the dumbest generation" (Bauerlein, 2008), and they are blamed for a widespread "narcissism epidemic" (Twenge \& Campbell, 2010). However, these broad brush strokes have largely been created by media and popular non-fiction accounts. One of the largest studies of this generation to date suggests that the infantilization of the Millennial generation derives not from their actual attitudes and behaviors, but largely because of the structural and systemic obstacles their generation has faced (Taylor \& Keeter, 2010).

This cohort has come of age through national events such as $9 / 11$, multiple wars, Hurricane Katrina, the Great Recession, and the Housing Crisis (Milkman, 2017; Thompson, 2013). While they are the most educated generation in history, this status comes at the cost of alarming levels of student loan debt (Thompson, 2013). These experiences have led to a decrease in mobility among the Millennial generation and many are finding themselves "stuck" and unlikely to move due to lack of economic prospects elsewhere (Fry, 2017). Moreover, structural barriers and cultural changes have informed this generation's overall hesitancy to meet traditional economic and social goal posts of American adulthood such as marriage and home ownership (Choi, Zhu, \& Goodman, 2018). But despite many setbacks, the Millennial cohort at large has developed a transformed vision of what they want their lives to be and how they want to live them (Taylor \& Keeter, 2010). In fact, they are more likely 
than any other generation to make personal lifestyle changes which reflect their views; of which they have many (Achieve, 2017; McDonald, 2015).

This context is significant to the present research environment, as it sensitizes the inquiry to the complexities of choice and possibility that may face young people living and working in Appalachian Kentucky (Carling, 2002). It also situates young adults' experiences within a particular set of cultural discourses surrounding the Millennial generation with which they must also contend. However, while this context is important to understand, this study refrains from using the Millennial label or adopting broad cultural stereotypes to frame the young adults in this study, as these often carry negative connotations and may not be relevant to the ways that young adults self-identify.

\section{Education and Work Experience}

This study further narrows its population of interest to young adults with some college education. Education not only drives migration choices, but it also gives young people more resources and opportunities to migrate (Black et al., 2011; Carr \& Kefalas, 2009). Focusing on those who have completed some college education contributes to our understanding of how such individuals identify with place and make commitments to applying their knowledge and skills toward community improvement (Dalsgaard Pedersen \& Gram, 2018). This is opposed to young adults who may have fewer chances to pursue life outside region and those who desire to leave but cannot (Black et al., 2011; Carling, 2005). Nevertheless, it is also recognized that those without college educations also have potential to contribute positively to their communities.

In addition to playing a role in migration decision making, college education is often both useful and necessary for obtaining positions in the areas of social, 
environmental, and economic change efforts illustrated in the previous section (ARC, 2015b). For the purposes of participant recruitment, type of work was conceptualized broadly in order to include myriad approaches and perspectives. The word "issues" was used in formal recruitment materials as opposed to specifying work aimed at economic, social, or environmental "justice," such that many different strategies and participants could be incorporated including those that do not frame their efforts in this way. Finally, "work" included both full and part-time employment, substantial volunteer efforts, civic involvement, and unpaid internships related to social, environmental, or economic issues within Appalachian Kentucky.

\section{History in Place}

Beyond current residence in Appalachian Kentucky, individuals were recruited based on having at least 15 years of residency experience in the region. This was informed by the need for explicitly local forms of action and leadership in Appalachian communities (ARC, 2015b; Hatch, 2009). Such history in place is notable for its contribution to strong place-based identities, local knowledge, and the social capital that facilitates entrée into community work (Fischer, Holm, Malmberg \& Straubhaar, 2000; Hatch, 2009; Humble et al., 2013; Morrone et al., 2015).

Research also suggests that strong bonds with place may inform both decisions not to migrate (e.g. Adams, 2016; Marshall et al., 2012) and community engagement (e.g. Bell, 2013; Smith, Davidson, \& Cameron, 2009; Vaske \& Kobrin, 2001). Length of residency was calculated cumulatively, rather than requiring that young adults continuously stayed in place over the last 15 years. Combined with a wide age range, this took into account that decisions to leave or stay evolve gradually and allowed for the possibility that some individuals had spent time away but chose to return (Carr \& Kefalas, 2009). 


\section{Establishing Significance to Social Work}

As demonstrated in this condensed overview, Appalachia is a complex region with experience of both catastrophe and rebirth (Williams, 2002). Social work has long played a role in its history. Early efforts occurred through settlement schools like those at Hindman and Pine Mountain in Kentucky; rural adaptations of Jane Addams's Hull House model of community engagement (Fisher, 1993). Later, popular nonfiction from the region (e.g. Caudill, 1962; Weller, 1965) was instrumental in the development of rural social work specializations (Ginsberg, 2005). The Appalachian region's fight for social, economic, and environmental justice has long offered insights into rural community organizing and activism (Rice \& Burke, 2018).

Often described in these accounts are the many obstacles to client services including transportation issues, limited access to health and social services, and fewer job opportunities (e.g. Ginsberg, 2011; Humble, Lewis, Scott, \& Herzog,2013; Lewis, Scott, \& Calfee, 2013; Stuart, 2014). Additional concerns include low population density, distance from urban centers, higher dependence upon natural resources, and a tendency to be somewhat controlled from a distance (Brown \& Schafft, 2011). Others cite generalized characteristics of rural populations that inform practice and personal life, such as suspicion of outsiders, lack of diversity, reluctance to seek help, and mistrust of psychological and governmental programming (e.g. Ginsberg, 2011; Humble et al., 2013; Phillips, Quinn, \& Heitkamp, 2010). Rural social workers are also acutely aware of such issues as dual relationships within the community, limits of confidentiality, working in isolation, and the need for a breadth of generalist knowledge where specialized services are unavailable (Daley, 2015; Edwards \& Addae, 2015; Humble et al., 2013). Moreover, those working in macro 
practice domains face another set of obstacles via the politically and ideologically divisive nature of the economic, social, and environmental problems they aim to address (Charania, 2011; Mudliar \& Arnold, 2017; Rice \& Burke, 2018)

Given these challenges, it is perhaps not surprising that many rural communities experience shortages of helping professionals and change catalysts like social workers (Mackie \& Lips, 2010). Of course, this too is impacted by the broader influences on population loss previously described. But it also results from the limited exposure and preparation students receive for rural practice (Daley \& Avant, 2014; Mackie, 2012; Mackie \& Lips, 2010; Mackie \& Simpson, 2007; Phillips et al., 2010). Although the National Association of Social Workers (2009) instructs educators to specify characteristics of rural communities and practice, there is ongoing need for both illustrative case studies and peer-reviewed scholarship to meet this demand (Belanger, 2014; Hickman, 2014; Scherch, 2015; Slovak et al., 2011).

Upon dissemination, the results of this study have a number of potential contributions in this regard. This includes implications for those in rural settings, but also applies to the need for all professionals to understand rural people and places as part of diversity. Among the most critical of these, the study contributes to the need for understanding the complex interactions between environmental, social, and economic issues and how social work might respond in rural areas (Slovak et al., 2011). Participants in this study have the potential to illuminate these connections both from the perspective of residents, but also as individuals who tackle these issues in their work (Pugh, 2007; Scherch, 2015).

Additionally, the specificity of a rural Appalachian context does not limit implications beyond its borders. Like many other places, both rural and urban, the struggle to stay in Appalachia is waged against the local effects of deindustrialization, 
international trade, environmental catastrophe, cultural and political division, and changing lifestyle expectations (Weinbaumm, 2004; Williams, 2002). In these ways, Appalachia represents just one microcosm of the rural residual of globalization (Martin, 2015); one of many "sacrifice zones" where the environmental, social, and economic well-being of residents is traded for the human and natural resources they provide toward the greater good (Bell, 2013; Fox, 1999; Cunningham, 2010; Reid \& Taylor, 2010). Or, in Eller's (2008, p. 8) words, “Appalachia’s problems are not those of Appalachia alone...We are all Appalachians.”

Thus, the experiences and work of young adults in this study can inform innovative strategies that aid organizations and communities in the retention and recruitment of young adults as part of broader efforts in development and social change. By illustrating commonalities of environmental, social, and economic oppressions, these findings can also bridge solidarity across justice movements and connect student learning in ways that transcend geography (Anglin, 2016; Rice \& Burke, 2018, p. 220). At the same time, the findings presented here honor the need for responding to injustice with locally led and inclusive efforts that build on the unique strengths and assets of each community (Humble et al., 2013; Mason, 2011; Riebschleger, 2007; Scales, Streeter, \& Cooper, 2014). It does so with a focus on the impact of this work on the individuals who are engaged in it (Daley \& Pierce, 2011). Although many participants in this study were not social workers by training, their experiences have the potential to inform and support students and practitioners as they navigate similar work in challenging environments. These emphases rests upon the belief that, as Jane Addams (1922, pg. 133) reflected, "social advance depends as much upon the process through which it is secured as upon the result itself." 


\section{Outline of Chapters}

This introduction serves as the foundation for each of the following chapters. It provided a rationale for the aims and scope of the study, covered necessary definitions, and outlined both persistent regional issues and implications for social work. Chapter Two presents the theoretical framework which aided in the inquiry's conceptualization and analysis, while Chapter Three organizes the qualitative approach and research design. Chapter Four describes the study sample, details the findings for each research question, and presents the context specific framework of doing place devised to envelop these. A discussion of results, implications for social work, strengths limitations, and suggestions for future research are offered in Chapter Five. 


\section{CHAPTER TWO: THEORY}

Maybe we need to come up with a different quality of life index for little country places like ours. How many points could we get for each hill? How much is a river worth? Can we add a category for walking on ground your ancestors walked? Or for the percentage of neighbors who'd show up in five minutes if you needed them, day or night? How can you measure that? And how can you measure how much you'd miss a place, if you had to leave?

-Residents of Owsley County, KY, HomeSong II

Over the course of several years, residents of Owsley County, Kentucky (2014) produced a musical theatre series entitled, HomeSong. Through this multigenerational project, performers shared stories based on oral histories and local culture, in part as a response to negative portrayals of Appalachian Kentucky in media and fiction (Pitts, Jr., 2014). As the quote shared above illustrates, a heartfelt takeaway from HomeSong was that there is meaning and value in place which cannot be captured by statistics.

It is fitting then that Anne Shelby (2014), a local author and HomeSong collaborator, chose these lines as part of her response to Annie Lowrey's (2014) article in The New York Times, which labeled the region the "smudge of the country" and declared it one of the hardest places to live. Based on metrics like poverty, unemployment, educational attainment, and obesity, Lowrey (2014) concludes perhaps the best investment is one that helps people leave. As demonstrated throughout history, this is not a novel response to the region's challenges (Martin, 
2015). Given the many economic, social, and environmental issues that persist, it is not uncommon for Appalachians to be asked, why not just move? (Catte, 2018).

In response, this chapter outlines a theoretical framework for place that views Appalachia as "a rich and complicated interplay of people and the environment...[freeing] us from thinking of it as facts and figures" (Cresswell, 2015, p. 15). Defined as a "meaningful location" (Cresswell, 2015, p. 12; see also Agnew, 1987), place is explored here for its potential to illuminate commitments to Appalachia, even where there are compelling reasons to go (Heinemann \& Hadler, 2015; Johnson, 2014). Place is also considered for how it may inform desires to engage in community issues and shape both the experience of staying and the meanings young adults' make of these connections.

Section One begins with a discussion of the importance of place. This is followed in Section Two with a review of key analytic domains of place relevant to this study. A third section provides an overview of symbolic interactionism as the lens through which these domains were viewed. Section Four outlines key interactive processes that shape place based experiences and meanings. Section five combines these elements into a framework of place as an interactive process using regional case examples. The chapter concludes with a summary of how this theoretical structure was applied within the study.

\section{The Importance of Place}

Over time, a number of theories have been applied to the Appalachian region in order to explain persistent disparities and influence the types of interventions promoted (Batteau \& Obermiller, 1983). In the 1960s, economic solutions were heavily informed by Oscar Lewis's (1959) culture of poverty hypothesis, which proposed that deprivation was caused by culture. Efforts were then made to change 
mountain traditions perceived as barriers to development (Eller, 2008). Other theorists (e.g. Caudill, 1962; Lewis, Johnson, \& Askin, 1978) framed Appalachia as an internal colony within the United States, emphasizing the region's economic and social exploitation by outside interests (Anglin, 2016). This approached Appalachia's challenges within a structural framework, removing personal blame and in some cases, empowering new models for Appalachian identity and action (Fisher \& Smith, 2016; Smith, 2016). Although they still enjoy popularity today, an emerging body of work now critiques the internal colony theory for its economic, cultural, and geographic determinism (Anglin, 2016; Billings, 2016; Fisher \& Smith, 2016; Simon, 1983-4; Smith \& Fisher, 2016; Walls, 1978).

While Appalachia has been defined, theorized, and represented in myriad ways, what each of these share is their attempt to connect people with place (Williams, 2002). Place is important to this study because it shapes individuals and their decisions and commitments, but also because these decisions and commitments shape places (Therborn, 2006). Interest in these aspects of place began in the 1970s as a response to sterilized geographic studies and accounts of physical environments that lacked emotional depth and connection to the people that inhabited them (Manzo \& Divine-Wright, 2014; Peet, 1998; Russell \& Ward, 1982). Attention to place has since increased as a result of globalization, enhanced mobility, virtual technologies, and the ongoing commodification of natural landscapes and resources, among other societal changes (McClay \& McAllister, 2014; Williams, 2008).

It is argued that, although these pressures seem to have a homogenizing effect on place, they may actually render it even more important in everyday life (Harvey 1996; Jacobson, 2002; Mander and Goldsmith 1996; McClay \& McAllister, 2014; Williams, 2008). As proposals for development and change are introduced, various 
stakeholders make claims regarding the value and meaning of place and how it should be used (Appleyard, 1979; Fisher \& Smith, 2012; Massey, 1993; Williams, 2008). Where modernity amplifies alienation, place may fertilize new forms of belonging, connection, and action (Billings \& Kingsolver, 2018; Robson \& Evans, 2010).

Place also plays an important role in developing locally relevant and informed approaches to social problems (Manzo \& Devine-Wright, 2014; Schambra, 2014). The establishment of the ARC for instance, may be seen as an example of place based policy making. Moreover, a significant number of scholars and activists use place as an organizing framework for both analysis and action in the region (e.g., Billings \& Kingsolver, 2018; Fisher \& Smith, 2012).

Although the benefits and values of place have been lauded, attention to place has also been posited as antiquated, overly nostalgic, insular and antithetical to the values of cosmopolitanism and the possible benefits of globalization (e.g., greater acceptance of diversity and humanism towards others; see Jacoby, 2014; McClay \& McAllister, 2014; Smith, 2018). This study presumes it is possible to embrace both; cherishing and building from what is uniquely local, while also embracing and learning from differences and commonalities beyond the boundaries of a particular place (Kingsolver, 2018; Mitchell, 2014; Pickles, 2018; Rice\& Burke, 2018; Smith, 2018). What is more, it acknowledges that, rather than being static geographic locations, all places have been shaped by forces at various scales over time (Cresswell, 2015; Rybczynaki, 2014; Williams, 2002).

\section{Key Analytic Domains}

Despite growing interest in the topic, scholarship of place often lacks theoretical substance, particularly outside of the discipline of geography (Tuck \& McKenzie, 2015). Where social science research attends to place, descriptions are 
often relegated simply to locations where data was collected; only rarely emerging as part of the analysis and interpretation (Basso, 1996; Massey, 1994; Tuck \& McKenzie, 2015). This is true for social work as well, where despite the field's hallmark person-in-environment perspective and ecological models, conceptual and theoretical orientations to place have received little attention in research and practice (Aguiniga \& Davis, 2014; Akesson, Burns, \& Hordyk, 2017). In attending to this gap, Akesson and colleagues (2017) suggest three components of place that are particularly relevant to social work including: place attachment, place identity, and territoriality. These were thus selected as key analytic domains to consider in this inquiry. Each concept is discussed in turn and in relation to the research questions and contexts that guide this study.

\section{Place Attachment}

Place attachment refers to the bond that develops between people and place (Altman \& Low, 1992). This bond arises as a result of a complex exchange of emotions and experiences (Akesson et al., 2017; Brown \& Raymond, 2007; Giuliani, 2003; Seamon, 2014; Smith, 2005). These interactions inform our sense of places present, memory of places past, and imagination of places future (Davidson, Bondi, \& Smith, 2005; Jones, 2005). Place based emotions can range from apathy to adoration (Relph, 1976, 2009; Seamon, 2014; Shamai, 1991). Likewise, place attachments cross a spectrum from weak to strong (Cross, 2015).

Place attachment can be seen as a dynamic process that evolves over time and in relation to changes in one's own life and also changes to places (Seamon, 2014). Migration, for example, can increase the number of places to which one experiences emotional bonding and increase the intensity of bonding to a single place (Barcus \& Brunn, 2010; Cuba and Hummon, 1993; Fielding, 1992; McHugh \& Mings, 1996; 
Gustafson, 2001b). This insight is supported by Barcus and Brunn's (2009; 2010) work among family reunion participants in Eastern Kentucky. Their studies reveal that place attachments can be maintained despite lack of continuous residence in an area (Barcus \& Brunn, 2009; 2010).

Research on place attachment demonstrates its connections to the primary areas of interest in this study. Place based emotions and experience may either drive people from, or draw them to a particular place (Davidson et al., 2005). Where immobility has been the emphasis of research, positive place attachment has been associated with lower likelihood of migration (e.g., Adams, 2016; Marshall et al., 2012; McHugh \& Mings, 1996; Fischer, Holm, Malmberg, \& Straubhaar, 2000). This functions not only because people are bonded with a physical location or its features, but also because place attachment encompasses relationships with people and culture, and can foster a sense of belonging (Low \& Altman, 1992; Nordström, 2014; Relph 1976).

Place attachment has also been identified as a significant component to social action and community engagement (Bell, 2013; Brown, Perkins, and Brown, 2003; Brown, Reed, \& Harris, 2002; Cuba \& Hummon, 1993; Manzo \& Perkins, 2006; Mesch \& Manor, 1998; Smith et al., 2009; Vaske \& Kobrin, 2001). Bell (2013) for instance, demonstrates how deep emotional connections to Appalachia play a role in why, and how, women fight for environmental justice in the region. Thus place attachment may inform young adults' commitments to working on social, environmental, and economic issues in their communities.

\section{Place Identity}

Place identity is closely related to place attachment (Cross, 2015). This domain refers to the idea that our bonds with place evolve alongside the development 
of the self (Bell, 2013; Hauge, 2007; Proschansky, Fabian \& Kaminoff, 1983; Twigger-Ross, Bonaiuto, \& Breakwell, 2003). Through the ongoing interaction between humans and their environments, sense of place and sense of self become intertwined in narrative, memories, and emotions (Akesson et al, 2017; Cross, 2015; Prochansky, 1983; Stedman, 2002). This process is ongoing, and identities often change due to life course developments or to changes to place (Denzin, 1992; McHugh \& Mings, 1996; Rubinstein \& Parmelee, 1992).

Although we may possess one overall sense of self, it is also accepted that this self may be comprised of multiple identities (Turner, 2011). Here then, place identity is examined as one component of the self, and this is viewed intersectionally, alongside others. This requires an orientation to the relationships between race, gender, class, age, work, religion, nationality, ability, and other characteristics, with the assumption that these impact how young people experience and make meanings of place (Hill Collins, 2015). Simultaneously, relationships and commitments to place are shaped by the many identities attached to places themselves (Altman \& Low, 1992). Thus, examining place identity requires looking at the complexities of 'self' at multiple levels (e.g. individual, regional) and from various positions (e.g., internal narratives, external discourses).

Identities are also carried with us as we move within and between places and through interaction. They can be performative and emergent, as contexts and relationships may demand varying degrees of openness around identities (Katovich \& Reese, 1993). This is documented in the way many Appalachians practice codeswitching; changing their speaking dialect to assimilate within a space where accents are stigmatized (Clark \& Hayward, 2013; Daniels, 2014; House, 2008). 
These connections are significant to this research context in a number of ways. First, people are often unlikely to move because where they are, is tied to who they are (Shen \& Gemenne, 2011; Mortreaux \& Barnett, 2009; Murphy, 2015), and a strong sense of self in place can translate to an associated sense of responsibility to service or action (Smith, Cameron, \& Bondi, 2009). Moreover, the development of identities and sense of self is considered a critical developmental task performed during adolescence and young adulthood (Erikson, 1968; Wolfe et al., 2017). This includes finding out who one is in relation to careers, beliefs, family and other critical decisions associated with decisions about place (Waterman, 1982; Wolfe et al., 2017).

\section{Territoriality}

A third analytic domain considered in this analysis is that of territoriality. Territoriality concerns the processes through which various entities lay claim to the land, people, and resources embedded in place (Akesson et al., 2016; Sack, 1983; 1986). Such claims are intimately tied to attachments and identities, as these impact what individuals and groups choose to do in and with places (Alkon \& Traugot, 2008). Here, territoriality is conceived as a domain that extends well beyond the material resources or features of a place. When various claims are made regarding place, these intersect with claims to identities, ideology, and other abstract qualities of social life that can be equally subject to controlling behavior.

The process of territoriality inevitably involves power (Gaventa, 2018). While place based experiences can give power to territorial action, places can also have power over people (Gaventa, 2018). Power dynamics within a place also regulate which symbols and meanings of place are harnessed for various agendas (Smith, 2018) and they can determine whose visions and desired actions actually get voiced and materialized (Alkon et al., 2008; Gaventa, 2018; Massey, 1994; Milligan, 1998). 
The concept of territoriality is critical to this study in that it assists in framing the tensions that arise within discourses about place and within plans to meet Appalachia's challenges. Including young adults in these conversations is an integral part of this process (Drake, 2001; Eller, 2008; Fisher, 2013; Fisher \& Smith, 2012; Kingsolver, 2018). As such, territoriality is approached here as a form of contestation that arises not only about how to proceed in the present, but also how to remember and interpret the past and how to conceive of the potential future of a place (Milligan, 1998). Attention to power is relevant in that it may inform whether and how young people engage with community issues.

\section{Symbolic Interactionism: A Way of Seeing Place}

Increasing interest in place has resulted in significant debate regarding definitions of place and the aforementioned analytic domains, relationships between these concepts, and potential applications (Cresswell, 2015; Manzo \& Devine-Wright, 2014). The study of place attachment, for example, has been approached by various disciplines and research methodologies (see Lewicka, 2011; Manzo, 2003; 2005; Manzo \& Devine-Wright, 2014; Patterson \& Williams, 2005; Trentlelman, 2009). Where quantitative studies operationalize, measure, and predict place attachment and its dimensions (e.g. Williams \& Vaske, 2003; Jorgensen \& Stedman, 2001; 2006), qualitative approaches have sought to understand place attachment as it is experienced by individuals and groups (e.g. Gustafson, 2001a; 2001b; Manzo 2005; Mazumdar \& Mazumdar, 2004).

Given this diversity, no single inquiry can contain all the complexity of people-place relationships (Manzo \& Devine-Wright, 2014; Patterson \& Williams, 2005). Thus, it is critical to clarify upon which theoretical tradition a particular study draws and to demonstrate fit with the research aims and environment (Patterson \& 
Williams, 2005). In this analysis, place is viewed from the theoretical perspective of symbolic interactionism.

Symbolic interactionism is a broad sociological theory which emphasizes the role individuals, groups, and society play in shaping the self, thoughts, and actions; as well as how these in turn, form society (Ritzer, 2011). It is also helpful for examining individual level processes including how thoughts about the self and ideas about how to act are formed through interactions with others (Blumer, 1969; Charmaz, 2014). With this emphasis upon interactive processes and the generation of meaning, symbolic interactionism is consistent with this study's research questions (see Chapter 1) and the qualitative approach adopted (see Chapter 3; see also, Brandenburg \& Carroll, 1995; Charmaz, 2014; Kotarba, 2014; Patterson \& Williams, 2005; Stokowski, 2002; Trentleman, 2009). Rather than offering predictive or causal properties, symbolic interactionism was used as a "way of seeing" place and interpreting the above mentioned analytic domains (Gusfield, 2003, p. 121; see also Blumer, 1969). This section offers background and a review of key principles of symbolic interactionism that facilitated this analysis.

\section{Background}

Early developments in symbolic interactionism were inspired by both pragmatism and social behaviorism (Dingwall, DeGloma \& Newmahr, 2012; Ritzer, 2011). For pragmatists, reality is created socially, individuals base their knowledge and interpretations of the world in what is practically useful, and understanding an individual requires examining their actions in the world (Charmaz, 2014; Ritzer, 2011). Social behaviorists emphasize that what makes humans unique is their capacity for language and thought, which then inform action (Ritzer, 2011). They are 
interested in both observable and unobservable behavior and insist that understanding these requires attention to both mental processes and social context (Ritzer, 2011).

Building on these foundations, George Herbert Mead (1934) laid the groundwork for symbolic interactionism in his pivotal text, Mind, Self and Society. For Mead, while the mind and self are critical to understanding human experience, these domains are preceded by and developed through our interactions in society (Ritzer, 2011). Later, Herbert Blumer (1969) coined the term symbolic interactionism and contributed attention to the processes through which individuals construct meaning through their social interactions (Ritzer, 2011).

\section{Basic Principles and Concepts}

These early influences have since been expanded and clarified through a number of basic principles and concepts. To begin, symbolic interactionism assumes that humans are uniquely capable of thought and posits that thoughts are developed through interaction (Blumer, 1969; Crooks, 2001). While great weight has been placed on face to face exchanges within symbolic interactionism, interactions also include those between the self, others, society at large, and social objects (Gusfield, 2003). Social objects can include tangible or material things, concepts, emotions, or any other aspect of social life (Lal, 1995).

Through these interactions, we learn the symbols needed to communicate our thoughts and begin to associate meanings with symbols and social objects (Blumer, 1969; Chang, 2000; Crooks, 2001; Gusfield, 2003; Ritzer, 2011). The meanings developed around objects and symbols subsequently inform how we behave toward them (Lal, 1995; Rosenbaum, 2009). Our behaviors in any given moment then, are intimately tied to what has been communicated to us in the past, our expectations of 
ourselves and others in that situation, as well as what we perceive to be their expectations of us (Cooley, 1902; Rosenbaum, 2009).

Within this interactional framework, the self is developed through social relationships and exchanges with individuals, social objects, and society (Blumer, 1969; Crooks, 2001; Lal, 1995). Through these interactions (e.g., socialization), we develop a sense of self in relation to others by receiving messages about values, norms, taboos, and other expectations (Rosenbaum, 2009). Over time, interpretations of the self come to have meaning to us and these have multiple layers (e.g., identities) and expressions (e.g., performances, roles; Crooks, 2001; Lal, 1995; Turner, 2011; Rosenbaum, 2009). This is an ongoing evaluative process that can act to verify or disqualify our internal sense of self (Turner, 2011).

Symbolic interactionism also maintains that humans have active agency over these expressions and that we choose if and how we adopt roles and adhere to norms (Turner, 2011). Here, it is important to recognize that stigmas can be attached to any one of these identities and roles based on our conformity with social expectations (Goffman, 1963; Ritzer, 2011). At the same time, the power to thwart and transform the social world is also inherent in our exchanges (Knauff, 2006).

\section{Limitations}

Despite the usefulness of these principles, a noted limitation of symbolic interactionism is that it has unsuccessfully delineated how broader social forces may determine our interactions (Gusfield, 2003; Ritzer, 2011). This study adopts Gusfield's (2003) approach, which insists that there are structural factors which have an impact on these relationships and their outcomes in any given time or place. Thus, individual agency occurs within a layered system of conditions and forces sometimes beyond our control or reach (Hill Collins, 2015). 


\section{Place-Based Interactions}

Symbolic interactionism assumes that symbols, meanings, self, and society are all co-constructed through emergent exchanges and relationships (Blumer, 1969; Crooks, 2001; Cross, 2015; Gusfield, 2003; Katovich \& Reese, 1993; Rosenbaum, 2009). As such, it holds potential for viewing place attachment, identity, and territoriality as interactive processes, rather than static conditions. Through these interactions, both people and places are shaped together (Therborn, 2006).

Building on a framework developed by Low (1992) and an understanding facilitated by symbolic interactionism, Cross (2015) outlines seven courses of interaction relevant to these developments. They include narrative, historical, sensory, spiritual, and ideological interactions, as well as commodification and material dependence (Cross, 2015, p. 502). Each is considered a dynamic process that evolves over time and occurs at multiple social levels (Cross, 2015). By viewing these through a lens of symbolic interactionism, this analysis is especially sensitive to the ways in which they occur through relationships with others and with society. Below, a brief description of each interactive process is offered . Although they are considered here separately, it is important to emphasize that they often occur in combination (Cross, 2015).

\section{Narrative}

Narrative interactions occur through storytelling, which includes any number of communicative processes and discourses (Cross, 2015). Narratives may be created from the top down, or the bottom up (Lal, 1995); originating internally or externally to the individuals, groups, and places of reference (Eller, 2001). For example, Appalachian stereotypes, myths, and untruths saturate external stories (Eller, 2001, p. $\mathrm{x})$, but exaggerated caricatures are also produced internally, such as through tourist 
attractions and gimmicks (Billings, 1999). Narratives are created with a variety of motivations and used for different purposes. Those that highlight challenges for instance, may bring important attention or funding to local initiatives (Billings, 1999; Wilson 1990).

\section{Historical}

The historical area of interaction refers to significant events in and relating to place, which then inform our emotional connections to it (Cross, 2015). Leaving the Appalachian region, for example, is a significant life course event for many young adults. Through interactions with others, they are often confronted with outsiders' perceptions of Appalachia while also recalling what they miss about home (WebbSunderhaus, 2016). These experiences may play a significant role in informing decisions around place and the development of place identity. Historical processes also extend beyond our personal history to include our genealogical or ancestral past in a place (Cross, 2015; Low, 1992). This analysis also considers references or concerns for future generations as a significant form of historical interaction.

\section{Sensory}

Sensory based interactions occur as we explore and experience place through our perceptions of sight, sound, taste, smell, and touch (Cross, 2015). One person's perceptions may be entirely different from another, as too might be the behaviors that are shaped in response (Gusfield, 2003). As humans, we also transform sensory experiences through our actions, both positive and negative, such as by changing what is seen, smelled, or heard in a place. For example, Appalachians of the Great Migration engaged in sensory interactions when they missed home, finding comfort in the continuation of traditional food and music (Drake, 2001; Eller,2008). 


\section{Spiritual}

According to Cross (2015), spiritual interactions are not necessarily religious or even otherworldy. Rather, they refer to the processes through which one develops a deep sense of belonging in a place, or the rootedness of their soul or spirit within a place. Farris's (2015) work exemplifies such connections in documenting the importance given by many Appalachian migrants to be returned to home and family cemeteries upon their death. Here, spiritual interactions also refer to developments that lead to a sense of purpose or calling to commit to a specific type of work or action in place.

\section{Ideological}

Ideological processes are embedded within the values and established morals of a place (Cross, 2015). These may be evoked formally, though laws and codes, or informally, through individual beliefs and actions (Cross, 2015). One learns the dominant ideologies of place through interaction with others and places come to be associated with these ideologies (Cross, 2015). Ideologies can be territorial and exclusionary, but they can also provoke actions to defend and reinvent places (Fisher \& Smith, 2012, p. viii). An example of both forms of ideological interaction presented themselves in 2017 when neo-Nazis and white supremacists staged a rally in Pikeville, Kentucky. The site may have been chosen based on the region's association with racist ideology, yet few if any residents actually participated in the event (Beckett, 2017). In fact, many people arrived wearing t-shirts printed with local artist Lacy Hale's mountain landscape and the tagline "No Hate in my Holler" in protest (Adams, 2017). Since that time, this phrase has become a rallying cry for many who want to rebrand the region's ideology as inclusive, demonstrated by the continued demand for Hale's items (Adams, 2017; House, 2018). 


\section{Commodification}

Commodification involves making decisions about place as a result of weighing various desirable and undesirable characteristics (Cross, 2015). These characteristics include resources, amenities, social supports, culture, and aesthetics, and the value of these is often developed through interaction. Commodification is an ongoing process and various components of place may be prioritized differently over the life course (Cross, 2015). Commodification is particularly relevant to the population of interest here, in that young adults often frame their place based decision in relation to expectations for career, family, and lifestyle (Black et al., 2011; Carr \& Kefalas, 2009; Florida, 2002; McLaughlin, et al., 2014; Shumway et al., 2014). Daniels (2014) highlights this struggle by outlining the myriad factors young Appalachians consider in their decision to stay in the region; a choice which sometimes requires sacrifice of basic needs like clean air and water (Daniels, 2014).

\section{Material Dependence}

The final course of interactive experience is material dependence. This refers to reliance upon resources or amenities of place (Cross, 2015). Level of dependence may change as resources increase or decrease, or as needs and wants evolve (Cross, 2015; Raymond, Brown, \& Weber, 2010). These conditions too are impacted by interactions with society at large and through important relationships. For example, parental expectations play a critical role in the desires of Appalachian youth to obtain college educations (Ali \& Saunders, 2011; Ali \& McWhirter, 2006; Chenoweth \& Galliher, 2004). Such messages are significant to consider in how they impact young people's ability or desire to move, or inform their capacity to engage in change efforts. 


\section{Synthesizing a Theoretical Approach to Place}

With an understanding that place is important, this chapter has described a number of concepts and principles that form the theoretical approach to place adopted in this study. These include key analytic domains in place scholarship, the principles and concepts of symbolic interactionism, and a number of relevant place-based interactive processes. Each of these were approached as components that assist in understanding the experience of young adults and their relationship to the Appalachian communities in Kentucky where they live and work. By adding perspectives like intersectionality and power, the study attends to limitations of symbolic interactionism and honors how these experiences may be tied to structures and cultures of choice and possibility that are stratified by identity, positions, and other markers (Carling, 2002; Castles, et al., 2014; Gustafson, 2014; Gray, 2018; Hill Collins, 2015).

Each of these interpretive elements were drawn upon as 'sensitizing concepts' during analysis (Blumer 1954, 1969; Bowen, 2006; Padgett, 2008). This is informed by the methods described in Chapter 3, which insist that theoretical dimensions be "held lightly and easily discarded" should that be warranted by the data (Padgett, 2008, p.14). This section demonstrates such applications through three case examples. Each example uses the vocabulary of this interpretive framework to describe an issue of economic, social, or environmental relevance to this study. As such, it orients the reader to the ways in which these concepts aided in the analysis of results.

\section{Prisons as Innovation: An Application to Economic Issues}

In 2018, plans were initially approved by Congress for a new prison to be built in Appalachian Kentucky; it's fourth since 1992 (Ryerson \& Schept, 2018). Reactions to this plan reveal how narratives and ideologies concerning economic progress are 
exchanged between individuals, groups and society. With a proposal to place the facility on a former coal mining site, the move was praised by some as an important part of repurposing the land, as well as its potential for job creation (Ryerson \& Schept, 2018). However, critics cited a number of issues including the many pressing regional needs that could be met with the half a billion dollars proposed for the project (Imperiale, 2017; Ryerson \& Schept, 2018; Williams, 2016). These differences represent territorial responses that draw on historical interaction and commodification, as benefits and consequences are weighed in relation to past, present, and future needs.

Frustrated by the business as usual approach and non-participatory processes evoked by formal leadership and politicians, many young people engaged in opposition (Catte, 2018; Fragoso \& Townes, 2016; Letcher Governance Project, 2016). In one instance, they protested during a SOAR innovation summit in Pikeville, $\mathrm{KY}$, where solutions for regional economic issues were discussed and the prison project was being celebrated (Letcher Governance Project, 2016). As Congressman Hal Rogers was speaking, protesters dropped a banner insisting "Prisons are not innovation" to reflect the silenced but growing resistance to prison building in the region (Letcher Governance Project, 2016). Here, a connection between identities, roles, and power is revealed by the ways in which each group articulated their territorial claims through symbolic interactions.

\section{Moving Mountains: An Application to Environmental Issues}

In the struggle against mountaintop removal mining, sensory interactions are often drawn upon to communicate threats to both the environment and place attachment. This is illustrated through Jolly \& Jones's (2015) vivid and emotional reaction to the sight of affected mountain ranges. They lament, "These are not small, 
innocuous plateaus; they are vast swaths of destruction, craters of giant proportions, and their presence is felt well beyond the now-extinct mountains of which they are painful reminders" (Jolly \& Jones, 2015, pp. 25-26). Similarly, Appalachian activist Larry Gibson (cited in Hedges \& Sacco, 2012, pp. 117-124) noted sensory changes through what he could no longer touch and what he now smelled as a result of mountaintop removal on his family's land. He reflected, "There was crawdads in the streams down at the bottom of the mountain. I could pick them out with my toes. Now nothing lives in the water. It stinks. Nothing lives on the land. And it's irreversible." For these and many others in the region, observable changes to place provoke strong desires to protect Appalachia's mountains.

Still, others may have entirely different perceptions and territorial responses to the same sensory experience (Gusfield, 2003). They may view the practice as fundamental to one's occupational identity or as a symbol of regional or even national pride. This can be seen in the widespread practice of purchasing "Coal Keeps the Lights On" license plates (Kentucky Coal Association, 2012). When displayed, these plates may be interpreted as symbolically communicating both a territorial stance on mining and associated personal identities.

The issue of mountaintop removal in the region is also framed by many larger cultural narratives, such as those that depict Appalachians as largely "antienvironmental, anti-state, and anti-science" (Rice \& Burke, 2018, p. 216). This has been facilitated by the politicization of environmental concerns and the ways in which political identities are tightly bound with other identities in the region, including religious. However, through faith-based environmental efforts, organizations like 'Christians for the Mountains' blur these binary framings and challenge perceived 
inconsistencies between being Christian and being concerned with the environment (Billings \& Samson, 2012; Davis, 2004; Feldman \& Moseley, 2003).

At the same time, faith-based environmentalists are often viewed with suspiciousness; including from within their own religious groups (Feldman \& Moseley, 2003; Jolly \& Jones, 2015). In order to navigate this highly divisive atmosphere, many Christian environmentalists avoid stigmatization and politicization by rejecting "activists" identities and by expressing their commitments through locally accepted norms and venues (Feldman \& Moseley, 2003; Fisher, 1993; Rice \& Burke, 2018). This dynamic elucidates the complexity of territoriality, attachment, and identity and the many interactive processes that may be experienced when confronting environmental issues within the region.

\section{Fabulachia: An Application to Social Issues}

As has been demonstrated, social interactions can attach stigmas to places like Appalachia (Wolfe et al., 2017). Similarly, social interactions can also stigmatize individual identities within a place (Goffman, 1963; House, 2008; 2014; WellsSunderhaus, 2016). In the case of LGBTQ youth in Appalachia, they may have to navigate both. First, narratives about rural places in general often ignore diversity across sexuality, gender, and other identities (Anglin, 2016; Eller, 2001; Gray, 2018). Due to such limited portraits, many young people may experience a sense of invisibility with the region and may even question their belonging or purpose therein (Daniels, 2014; hooks, 2014; House, 2008; 2014). Writing of such experiences in the region, McMichael (2014, p.142) writes, "Daily invisibilities aren't a brutal beating; instead, they mark the lives of those who are different with a thousand paper cuts."

These narratives also occur alongside exclusionary ideologies and historical experiences of rejection, abuse, and violence attached to identity and expression (Carr 
\& Kefalas, 2009; Daniels, 2014; McMichael, 2014; Well-Sunderhaus, 2016).

Combined, these narratives and experiences have tremendous potential to inform place attachment and place identity. Through interactive processes like commodification, LGBTQ youth must weigh their safety, well-being, and the potential to flourish in a place (Daniels, 2014). In the end, many may feel they have little choice but to leave (House, 2014; McMichael, 2014).

Still, others may be empowered to stay, claiming their multiple identities and increasing LGBTQ visibility in present spaces and through reclaiming past and future narratives (Fischer \& Smith, 2012). This form of territorial resistance is demonstrated in Gray's (2018) ethnographic research with LGBTQ youth in rural Kentucky. Gray (2018, p. 121) draws a complex picture of lived experience through which young people contested oversimplified constructions of queerness as a "city thing" through everyday interactions. She reflects, “...young people knew how to occupy spaces that were not entirely their own, but that still gave them room to walk that fine line between being familiar - 'you know me, I'm so and so's son or daughter' - and also being fabulously queer" (Gray, 2018). Such resistance also occurs through language and symbols, like the trend of adopting the term "Fabulachia" to refer to LGBTQ folks in the region (House, 2018). This example reveals the importance of honoring the intersectionality of identities among those who stay. It also reveals the power of everyday modes of territoriality where narratives and ideologies perpetuate stigmas.

\section{Summary}

This section provided regional case examples of contemporary social, economic, and environmental issues as illustrative of the context facing young adults in Appalachian Kentucky. Each of these highly contextualized examples provides insight into how the sensitizing concepts of place identity, attachment, and 
territoriality can be used to interpret young adults' experiences in the region. Moreover, they illustrate uses of symbolic interactionism and the importance of emphasizing how meaning and action evolve through relationships in the region. The final section below summarizes the study's overall approach to the use of theory.

\section{The Place of Theory}

Theory plays an important role in framing social problems and positioning them for the purposes of research and action (De Poy \& French Gilson, 2007; Payne, 2014). However, various perspectives have emerged regarding the function of theory within qualitative inquiries (Padgett, 2008). It is argued that overuse of theoretical frameworks can drive data collection and analysis in premature directions (Glaser, 1978; Padgett, 2008; Ramalho, Adams, Huggard, \& Hoare, 2015). At the same time, thorough analysis requires familiarity with literature surrounding the phenomena of study (Glaser, 1978). Thus it would be naïve for qualitative researchers to claim they enter inquiries free from the influence of existing theories (Padgett, 2008).

According to Padgett (2008), one way to resolve this dilemma is for qualitative researchers to identify the uses of theory within various stages of the research process. In this study, place and symbolic interactionism were used as theoretical lenses that informed the overall aims, research questions, and data collection strategies. The various elements of place were also used as sensitizing concepts during analysis (Blumer, 1954, 1969). The following chapter describes these methods in detail and illustrates their connection to this theoretical foundation. However, overall, this approach maintained a balance between overuse and lack of theoretical perspective (Padgett, 2008). 


\section{CHAPTER THREE: METHODS}

Individual research questions direct the selection of particular methodological approaches and techniques (Silverman \& Marvasti, 2008; Valentine, 2001). The questions posed in this study support the selection of a qualitative method of inquiry because they seek an in-depth understanding of an experience that is little researched, and are concerned with gaining emotional depth and understanding from the perspective of insiders with direct experience (Dwyer \& Limb, 2001; Padgett, 2008). Constructivist Grounded Theory ([CGT] see Charmaz, 2003; 2006; 2014) was chosen as the method of qualitative inquiry for its ability to generate a context specific interpretive framework illustrating the experiences of younger adults staying and working in Appalachian Kentucky. As this method has potential to inform existing theories, policies, and practices (Charmaz, 2014), it is well suited for research in the field of social work.

This chapter begins with a brief overview of CGT, followed by the epistemological, ontological, and theoretical foundations that inform this approach. The subsequent section outlines the techniques of data collection and analysis that comprise the research design. Concluding the chapter are sections dedicated to trustworthiness and the process of writing and dissemination, respectively.

\section{Constructivist Grounded Theory}

CGT is a qualitative approach to research that informs both data collection and analysis (Charmaz, 2014). CGT arose out of earlier formulations of the Grounded Theory Method (GTM) beginning with Glaser and Strauss's (1967) The Discovery of 
Grounded Theory. Rather than testing existing theories through the collection and analysis of data, Glaser and Strauss (1967) proposed a comparative method that generates theories from the data itself (Padgett, 2008). In doing so, Glaser and Strauss (1967) contributed to the formalization of qualitative analysis through the identification of induction as a system of logic unique to qualitative research and by outlining systematic strategies to employ during analysis (Charmaz, 2014).

Later, Charmaz (e.g., 2003; 2004; 2014) formalized CGT as a permutation of grounded theory informed by postmodernism. According to Charmaz (2006; see also Padgett 2008), a primary difference between the two versions of grounded theory is that the original goals of GTM included to eventually explain and predict phenomena (see Glaser, 1978); while CGT emphasizes the generation of an interpretive framework. Rather than 'discovering' a grounded theory within the collected data, CGT acknowledges that through interaction with the participants, their environments, the data provided, and the researchers' own subjectivities, the theory or framework is 'constructed' collaboratively through the research process and out of the data itself (Berthelsen, Lindhardt, \& Frederiksen, 2017; Silverman \& Marvasti, 2008).

CGT emphasizes meaning and action, and seeks to understand participants' worlds through their own language, values, and perceptions, while also requiring the researcher's transparency and reflexivity (Charmaz, 2014). CGT insists that despite temporary conclusions, the meanings, perceptions, and values collected will continue to evolve (Charmaz, 2014). Thus the final product is accepted as an interpretive portrait in time, rather than a static and neutral description (Charmaz, 2014).

\section{Epistemological, Ontological, and Theoretical Foundations}

Critical to any research endeavor is beginning with clarity about the epistemological, ontological, and theoretical foundations which inform and guide the 
study (Padgett, 2008). With these foundations established, the particular

methodological approach is rooted within a clear philosophy and body of guiding principles (Padgett, 2008). The CGT approach rests upon constructivist epistemology and ontology, and is situated within the theoretical premises of both pragmatism and symbolic interactionism. This section provides a brief overview of how these foundations guide the CGT methods employed in this study.

\section{Ontology}

Constructivism is not interested in demonstrating that social constructs (e.g., place) and their consequences (e.g., displacement) are not real from an ontological stance (Gergen, 2009; Padgett, 2008). Rather, within a research methodology, constructivism encourages researchers to detach themselves from the current reification of social constructs (Padgett, 2008). This allows for understanding the processes through which concepts and objects are defined, enacted, contested, and reborn over time and through a particular research setting and its contexts (Padgett, 2008). For example, setting aside pre-existing conceptualizations about Appalachia allowed me to recognize and emphasize the various ways in which participants created their own meanings associated with the region and their location therein.

\section{Epistemology}

Epistemology refers to the study of knowledge and knowledge creation, and these concerns heavily inform the CGT process (Charmaz, 2017). The constructivist lens inspiring CGT asserts that knowledge is created as people interpret and assign meaning to objects and experiences (Breckenridge, Jones, Elliot, \& Nicol, 2012; Crotty, 2013; Guba \& Lincoln, 1994; Hayes \& Oppenheimer, 1997; Pidgeon \& Henwood, 1997). The overall aim is not to measure or capture a singular truth, but to interpret the meanings made by a particular group of people in a specific place and 
time; with the coinciding understanding that there are multiple perceptions of reality connected to the particular topic of interest (Breckenridge et al., 2012; Charmaz, 2003; Mills, Bonner, \& Francis, 2006). Constructivist epistemology informs this research through a commitment to accept the co-constructed nature of meaning that develops through data collection and analysis and to honor the final outcome as a product of the participants' meanings as interpreted through my own lens as the researcher (Mills et al., 2006).

\section{Theoretical Frameworks}

In Chapter 2, symbolic interactionism and pragmatism were reviewed as theories which informed my approach to the sensitizing concepts of place. As philosophical underpinnings, these also informed the development of CGT methodology (Charmaz, 2017). Discussed here are the ways in which pragmatism and symbolic interaction informed the research methods.

According to Charmaz $(2008 ; 2014 ; 2017)$, pragmatist philosophy adds to CGT by locating experiences and actions in context, focusing on processes and change, and joining value and facts through interpretation. These pragmatist elements inform the design and implementation of this study through the incorporation of the subjectivities of the researcher into the analysis and valuing the abilities of participants to construct their own meanings and interpretations from their experiences (Charmaz, 2014). Tenets such as the emphasis upon action, processes, and change were articulated through data collection and analysis strategies which sought to understand these specific elements in the context of participants' experiences with migration, resisting migration, and their connections to the work they are performing in their communities. 
Symbolic interactionism is also deeply embedded throughout the CGT methodology used in this study, which is essentially takes a theory-method combination in its approach (Charmaz,2014). Its values were adopted in the collection and analysis of data such as through emphasis upon participants' language, actions, interactions, and meanings (Ritzer, 2011). Additionally, significant effort was made to understand the broader context within which participants and their experiences are situated, with an understanding that these inform the meanings and actions taken on by each individual (Charmaz, 2014; Clark, 2005).

\section{Research Design}

A research protocol informed by the abovementioned foundations was developed and approved by the University of Louisville Institutional Review Board (see Appendix A). This section outlines the data collection and analysis strategies employed within this study. As qualitative researchers are constantly in confrontation with issues of ethics, (Charmaz, 2014; Soobryan, 2003), these must be attended to throughout the research process (Shaw, 2008). Therefore, matters of research ethics are discussed as they arise within each stage or process.

\section{Data Collection}

CGT asks that the accounts given are provided significant context to aid in that understanding (Charmaz, 2014; Clark, 2005). In order to provide context beyond what is available through a single data source or through available literature, this study invokes the use of three primary techniques of qualitative data collection: interviewing, observation, and document review (Padgett, 2008). This approach allowed for triangulation of data from multiple sources in order to increase the depth and scope of understanding in service to the research questions (Valentine, 2001; 
Wengraf, 2001). A rationale for each of these strategies is shared below, alongside inclusion criteria and procedures for their implementation.

\section{Interviews.}

Qualitative interviewing allows for the collection of diverse experiences that illuminate the social processes identified by a study's research questions (Rubin \& Rubin, 2012). This study employed an intensive interviewing strategy aimed at "thick description" (Geertz, 1973) of participants' experiences, meanings, actions, and processes related to their lived experience. The overall approach to the interview process was to maintain consistency across interviews while remaining flexible enough to honor the iterative nature of the CGT process (Charmaz, 2014).

\section{Inclusion criteria and recruitment.}

Five inclusion criteria were established for individual interviews, each informed by the research questions and relevant literature and theory reviewed in Chapter One. Their specificity is justified in that the purpose of qualitative research is not to generalize to the larger population, but rather to acquire in-depth understanding of a particular phenomenon from a group of individuals that share common experiences (Padgett, 2008). First, all participants currently lived in an Appalachian county of Kentucky. This requirement adopted the ARC's (2009) geographic boundaries, which includes 54 counties in the eastern part of the state. Second, participants lived in the region for 15 years, cumulatively. This accounted for time away for education and other pursuits and emphasized individuals with social and cultural capital and significant experiences in the region. Third, this study recruited individuals based on an expansive definition of the Millennial generation, which includes young adults born between 1980 and 2000 (see Stein, 2013). A fourth criteria maintained that all participants had completed some college education. This meant 
either the successful completion of a degree, current enrollment, or past enrollment in a degree program but lack of current effort toward the degree. Finally, young adults in this study were each required to be working in areas of social, economic, and environmental importance in the region. This was purposefully left broad to allow potential participants to self identify as working in these areas.

Recruitment materials were sent to organizations and individuals who appeared to align with the conceptualization of economic, social, and environmental issues in the region. Economic issues included such activities as entrepreneurship, regional development initiatives, downtown revitalization programs, tourism, and direct work with individuals and families in poverty or experiencing unemployment. Environmental issues included activism and advocacy against practices like hydraulic fracturing and mountaintop removal mining, sustainable agriculture, conservation initiatives, and forest management. Social issues were conceived as involving many other challenges in the region including substance use, mental and physical health disparities, lack of educational parity, and issues related to diversity, inclusion, and representation of the region itself. To qualify as working in these areas, participants were required to be either working full or part-time or engaged substantially through volunteer efforts, membership in civic groups, or unpaid internships. Ultimately, individuals who received the recruitment flyer (Appendix C) determined whether their work fit the conditions of the study based on this definition.

Purposive snowball sampling was used to recruit participants who met inclusion criteria for interviews. Snowball sampling is useful when or if it seems that members of a particular group or network will not be readily willing to participate without referral or suggestion from a trusted other (Padgett, 2008). Given the long 
history of exploitation and misrepresentation by outsiders to the region, this was deemed an important ethical approach to recruitment for this study.

Sampling began with the common approach of using my existing contacts and networks to make connections for the study (Silverman \& Marvasti, 2008). I also identified critical gatekeepers within the region. Gatekeepers are individuals who control or regulate access to certain individuals, spaces, activities, documents, or other sources of potential data and are often needed in the stage of recruitment and rapport building (Hammersley \& Atkinson, 2007; Padgett, 2008). Additionally, potential participants were identified through outreach to relevant organizations within the region and by asking gatekeepers to connect their members through social media, email, or by inviting the researcher to meetings or events for the purposes of recruiting participants. Where professional email addresses were made publicly available through organizational websites, individuals with job titles appearing to pertain to this study were also emailed individually using a standardized script (see Appendix B) and attached recruitment flyer (see Appendix C).

Gatekeepers and individuals targeted for this research were those whom I was able to identify based on their efforts working on economic, social, or environmental issues within various organizations based in or serving Appalachian Kentucky. The search for such organizations and individuals was initially based on personal knowledge and experience. Additionally, eligible participants were identified via Google searches for organizations and individuals active in work surrounding current issues in the region. These included regional chapters of Kentuckians for the Commonwealth (KFTC), Mountain Association for Community and Economic Development (MACED), local county Health Departments and local county offices of the University of Kentucky Cooperative Extension Service, Appalshop, Hindman and 
Pine Mountain Settlement Schools, the Family Resource and Youth Service Centers at public schools, as well as active clubs or organizations within the region's college and university campuses. Identification of additional groups and individuals evolved through searching relevant social media accounts, newspaper articles related to regional issues, and by word of mouth with participants or leaders within the already identified organizations. Participants were also recruited through attendance at regional events and gatherings as part of the participant observation process described below. Finally, as participants were recruited, I also asked for their assistance locating additional candidates for the study and several participants were identified in this manner.

\section{Interview procedure.}

While gatekeepers were needed to arrange initial contact with some participants, I made direct contact with each potential participant and introduced the purposes of the study. This avoided gatekeepers being responsible for communicating the purposes or processes of the study (see Seidman, 1991). Once participants agreed to participate, individual interviews were scheduled. Every attempt was made to meet participants within their county of work or residence. In person interviews $(n=26)$ were arranged with each participant at their convenience and in locations that were comfortable for participant (see Seidman, 1991). Locations included the participant's place of employment $(n=9)$, local restaurants or coffee shops $(n=9)$, and spaces within organizations arranged by gatekeepers or other participants $(n=8)$. In some cases, inperson interviews were not possible and telephone interviews $(n=4)$ were conducted due to distance, availability, or participant preference. Although little research has been conducted to evaluate differences between formats, telephone interviews are supported particularly in the event that interviewees live at a long distance from the 
interviewer and for follow-up interview after a prior in person meeting (Padgett, 2008).

Both phone and in person interviews were digitally recorded. Having access to recordings benefits researchers in that they offer large volumes of detailed data in their originally shared format, maintaining the integrity of the data independent of the researcher's memory (Silverman \& Marvasti, 2008). In person interviews were recorded with both a Sony ICDUX560BLK Stereo Digital Voice Recorder and through Rev Voice Recorder(C, an audio recording application available for iPhones through Rev.com, Inc. (2018). Phone interviews were recorded using the TapeACallC (TelTech, 2018) iPhone application.

The Institutional Review Board (IRB) for this study approved a verbal consent process for individual interviews. Prior to each interview, participants received an electronic version of the statement of consent (see Appendix D). In-person interviewees were additionally provided a paper copy of the consent. Key to the ethical integrity of the qualitative research process, the consent document: disclosed my university affiliation and contacts for both dissertation chair and IRB, described the purpose of the study and the specific research questions; maintained that strict confidentiality and privacy would be kept of all data; assured participants that their participation was voluntary; explicitly stated there was no incentive for participation; and noted that to my knowledge, there were no risks for participating in the study. (Padgett, 2008).

I began each interview by restating the purpose of the study and allowing the participant to voice any concerns or questions prior to beginning the interview. I then reviewed the informed consent prelude that described the conditions of the study. Verbal consent was gained from each participant either in person or by phone, by 
asking that they state that they understand and accept these conditions. I informed participants that allowing for audio recording was voluntary and asked if they agree to allow recording to continue for the remainder of the interview.

\section{Interview guide.}

Following the informed consent process, data was collected for each participant specific to their fit for the inclusion criteria. This included: year of birth, years of education, county of residence, and place(s) of work and civic involvement. Age, race and gender were also recorded as self-described by participants.

Interviews followed a semi-structured interview guide (Table 1) that used open ended stem questions to gently direct the conversations toward the aims of this research. Drawing on Seidman's (1991) three stage life history interview and sensitizing concepts of place, interviews sought a chronological understanding of personal timelines specific to living in and outside of Appalachia and working on social, environmental, and economic issues in the region. Interviews also aimed at indepth understanding of the meaning participants made of these experiences. This approach was informed by the Pragmatist foundations of CGT, which honor individuals' agency in creating and interpreting the meanings they associate with their experiences (Charmaz, 2014). For example, Question Four asked, “What is the connection between your decision to stay here (in the area/region) and your commitment to this work?" Symbolic interactionism also informed the interview guide, in that attention was paid to how these meanings and experiences evolve through social exchanges with others (Charmaz, 2014). This was accomplished through a number of clarifying and exploratory questions that ask participants to describe how relationships impact their experiences of both staying and working on 
issues (e.g., What is your relationship to other people working on this issue? Who was

influential to your decision to stay?)

Table 1. Interview Guide

\begin{tabular}{|c|c|}
\hline Initial Prompt & Clarifying and Exploratory Questions \\
\hline $\begin{array}{l}\text { Question 1. I'd like to start } \\
\text { by asking you to you share a } \\
\text { little about yourself... }\end{array}$ & $\begin{array}{l}\text { Where were you born? } \\
\text { What other places have you lived in and for how } \\
\text { long? } \\
\text { Do you have friends and family here? } \\
\text { Do you have friends and family who have moved } \\
\text { away? } \\
\text { Tell me about your current relationships? } \\
\text { Tell me more about your personality, talents, skills, } \\
\text { your strengths, your challenges... }\end{array}$ \\
\hline $\begin{array}{l}\text { Question 2. Could you say } \\
\text { more about your experiences } \\
\text { living in this area... }\end{array}$ & $\begin{array}{l}\text { How would you describe this community? } \\
\text { What was it like growing up in this area? } \\
\text { Have you ever lived outside the region? } \\
\text { What experiences or circumstances led to that move? } \\
\text { Who was influential to your decision to stay? What } \\
\text { was influential? } \\
\text { What positive changes occurred as a result of } \\
\text { leaving? Any negative? } \\
\text { Looking back, what led you to return? } \\
\text { Who was influential to that decision at the time? } \\
\text { What was influential? } \\
\text { What positive changes occurred as a result of } \\
\text { coming back? Any negative? } \\
\text { How have your perceptions of the area/or the region } \\
\text { changed over the course of your life so far? } \\
\text { Now that you are living and working here, what are } \\
\text { some of the challenges you experience day to day.... } \\
\text { How do you cope with these? } \\
\text { What people are important in coping with those } \\
\text { challenges? What resources? What values? What } \\
\text { beliefs? } \\
\text { Do any of these challenges push you to consider } \\
\text { leaving the area/region? } \\
\text { If so, where do you think you would go? What } \\
\text { attracts you to that place? What resources? What } \\
\text { values? } \\
\text { If not, what does it mean to you to stay put here, } \\
\text { even despite these challenges? } \\
\text { Looking ahead, how long do you see yourself } \\
\text { staying in the area? }\end{array}$ \\
\hline $\begin{array}{l}\text { Question 3. Could you tell } \\
\text { me more about the work you } \\
\text { are doing on/with }\end{array}$ & $\begin{array}{l}\text { How did you become involved in this work? } \\
\text { What experiences contributed to your interest in this } \\
\text { issue? }\end{array}$ \\
\hline
\end{tabular}




\begin{tabular}{|l|l|}
\hline issue/organization? & $\begin{array}{l}\text { What is your role in the organization? } \\
\text { How did you prepare for this role? } \\
\text { How are your relationships with the other people } \\
\text { working on this issue? } \\
\text { What are the challenges inherent in the work? } \\
\text { What are the benefits? } \\
\text { What progress have you witnessed? } \\
\text { How does this work impact your personal } \\
\text { relationships? } \\
\text { How does this work impact your role in the larger } \\
\text { community? } \\
\text { What impact has this work had on your community? } \\
\text { Where do you see this work headed in the future? } \\
\text { Do you see yourself continuing this work in the } \\
\text { future? } \\
\text { What are the current threats to this work or its } \\
\text { impact? (e.g., ideological, economic, political) }\end{array}$ \\
\hline $\begin{array}{l}\text { Question 4. What is the } \\
\text { connection between your } \\
\text { decision to stay here (in the } \\
\text { area/region) and your } \\
\text { commitment to this work? }\end{array}$ & $\begin{array}{l}\text { What else should I know to understand this } \\
\text { connection? } \\
\text { What is the difference between people who stay and } \\
\text { those who leave? } \\
\text { What tensions do you experience as a result of } \\
\text { staying? } \\
\text { What advice would you give to young people in } \\
\text { similar circumstances? }\end{array}$ \\
\hline $\begin{array}{l}\text { Question 5. Is there } \\
\text { something else that has come } \\
\text { you during the interview that }\end{array}$ & $\begin{array}{l}\text { See Below. } \\
\text { yould like to share? }\end{array}$ \\
\hline
\end{tabular}

In addition to these questions, generic exploratory or probing questions were used for moments where clarification or elaboration was desired. While not necessary for all interviewees, probes are used in many cases to encourage the participant to continue exploring various topics or earlier questions (Padgett, 2008). This is approached with the goal of reaching what Wengraf (2001, pg. 6) calls 'depth 
realities.' The use of exploratory questions (e.g., Can you describe what you mean by ?) assists in this, as they required challenging my own assumptions and complicating concepts or topics that appear simple on the surface.

Although the interview process followed the semi-structured guide, each interaction evolved into a dialogue between the participant and myself, consistent with the grounded theory methodology (Padgett, 2008). Moreover, while the established questions were necessary to the early stages of this analysis, it was also necessary to introduce new questions and directions as the analysis proceeds and new insights were gained (Charmaz, 2014). Moreover, in later stages of data collection, I moved from the background to the foreground in my role as interviewer. During theoretical sampling, for example, I began to explicitly ask participants to reflect on established codes and processes developed from earlier interviews.

\section{Ethical considerations.}

The nature of the information shared, the interview process, and the relationship built with participants pose ethical questions in qualitative research (Padgett, 2008). With each interview, I made effort to avoid leading questions and interruptions, listen attentively, and emote empathy (Charmaz, 2014). I monitored body language and tone, and sensitively guided the interview (Padgett, 2008). At times, my reading of participants encouraged me to take a stance of mutuality and closeness characteristic of feminist or participatory approaches to research (Padgett, 2008; see also Oakley, 1981; Salmon, 2007). This issue arose in circumstances where individuals appeared nervous or where sensitive information about vulnerable identities was shared. I responded through impression management and a conversational style appropriate for such situations (Hammersley \& Atkinson, 2007; Padgett, 2008). 


\section{Participant observation.}

In conducting participant observation, a researcher enters settings where the individuals and groups of interest are active, and then goes about becoming part of the setting (Bonner \& Tolhurst, 2002; Hammersley \& Atkinson, 2007; Watts, 2011). The practice allows for in situ exchanges between the participants and the researcher, and provides opportunities for the researcher to witness actions, processes, and interactions in real time (Agar \& McDonald, 1995; Berthelsen et al., 2017). The researcher gathers data by watching, doing, and asking questions about what is being observed or experienced, and through extended relationships and interaction (Bonner \& Tolhurst, 2002).

While interviews focus on the importance of what people say, the method of participant observation moves closer toward an analysis of what they do (Spradley, 1980). Participant observation allows the researcher to witness discrepancies and alignments between what individuals say and do, as well as to observe how they interact with others (Laitinen, Kaunonen, \& Åstedt-Kurki, 2014; Padgett, 2008). The primary differences between being a typical participant in a setting and being a participant observer include: the dual purpose and simultaneous role as insider and outsider, explicit awareness of the need to note the rules of behavior and other details, emphasis upon heightened introspection or reflexivity on the part of the observer, and record keeping (Spradley, 1980).

Participant observation aligns well with CGT's foundations in symbolic interactionism, which emphasizes how meaning develops through interactions, language, and symbols (Berthelsen et al., 2017; Charmaz, 2014). Participant observation strengthens these aims by adding data that moves beyond the linguistic or verbal meanings (Aagard \& Matthiesen, 2016), such as by giving opportunity to see 
how these meanings may be enacted or negotiated with others. This is significant as people express meaning and agency through words, but also through behaviors and interactions with people, places, and objects (Hammersley \& Atkinson, 2007).

The incorporation of participant observation into this methodology also supports CGT's emphasis upon understanding interview data in the context of the broader circumstances surrounding individual lives and the issues facing their communities and work (Hammer, Fletcher, \& Hibbert, 2017). Although contextual information can be gained in ways outside of observation (e.g., reading local news, through literature reviews), simply adding the study of contexts to interview data is not sufficient for tracing broader forces like cultural representation and how they operate within the environment(s) of study (Hook, 2001; see also Aagard \& Matthiessen, 2016). Such insight requires entry into the spaces where this work is happening and examination of the physical and material arrangements therein, such as who is given a platform for speaking and other privileges (Brinkmann, 2014).

\section{Selection criteria.}

Selection of settings for participant observation required making informed decisions about the types of locations, activities, or events that would generate appropriate data and insight into the research questions (Hammersley \& Atkinson, 2007). Generally, locations were identified as they related to the work of individual interview participants, the work of their organization, or to further understanding the broader social, economic, or environmental issues which participants noted addressing through their work. Once rapport was established with participants through interviews, I also asked for their permission and suggestions for attending meetings or events related to their work in the region. In addition to decisions related to content and analysis, practicalities of site selection included criteria common to all participant 
observation such as simplicity, accessibility, potential impact of my presence, the frequency of activity, and opportunity for participation (Spradley, 1980).

In total, 74 hours of participant observation were conducted for this study. In addition to frequenting local businesses in the region, locations of observations included a Poor People's Campaign event in Lynch, Kentucky, an environmental rally at the Kentucky State Capitol Building, an environmental teach-in on fracking pipelines, a community clean-up, a downtown revitalization and community event planning meeting, and a student potluck for Appalachian students hosted by a studentled college organization. I also participated in three days of the Appalachian Studies Conference and an all day Tedx event in Corbin, Kentucky; both of which highlighted themes relevant to this study and were attended by multiple participants in this study.

Following an initial period of general observations of groups and spaces, the process of participant observation moved beyond description toward answering specific questions and resolving analytic dilemmas (see Spradley, 1980). This resulted in narrowing the scope of observations to a geographic area where several participants lived and worked, and to a single organization where multiple observations could be made in connection to the study's aims. The choice of a community and organization comprised of existing participants allowed me to observe how these individuals navigated interactions, and embodied the values and meanings shared through interviews through their actions (Lichterman, 1998). Being present across multiple spaces and points of time within these sites also allowed for witnessing changes across spaces, times, and interactions (Spradley, 1980).

\section{Covert versus overt observation.}

Among the most significant ethical considerations with regard to participant observation is the decision to conduct oneself overtly or covertly (Whyte, 1979). 
While covert participation observation may introduce the researcher to more 'authentic' or candid interactions, it poses significant ethical challenges into the research process and can be emotionally disturbing to both the researcher and participants, should they later learn of what transpired (Li, 2008). Given the dilemmas and tensions of covert participant observation (McCurdy \& Ulman, 2014; see also Lauder, 2003; Lichterman, 2002), where at all possible, participant observation in this study was done overtly and with the permission and or invitation of participants in this study or other gatekeepers.

Although every effort was made to notify leaders and gatekeepers of my attendance at various meetings or events, there were instances when not all participants in those settings were aware of my purposes. For example, exceptions included when I attended a public event or open meeting despite lack of response from emails soliciting a blessing for my attendance from gatekeepers, or where events or activities were open to the public and so large that there was no readily identifiable person. This is an oft cited challenge inherent to participant observation (e.g., Moore \& Savage, 2002; Zahle, 2017), given it is simply impossible to notify everyone of your study (Padgett, 2008).

Participant observation done in public spaces relies upon a general understanding that anyone has the right to do so (Spradley, 1980). However, in alignment with ethical guidelines on the practice, the privacy of individuals and groups within those spaces was maintained throughout the writing and dissemination of this research (Spradley, 1980). This is considered an ethical way to manage this ambiguity of consent, particularly given the nature of the observations made in this study were not recording or reporting on stigmatized behaviors a practice which has 
the potential to be interpreted as a more deceptive form of covert data collection (Padgett, 2008).

\section{Active versus passive participation.}

As in other methodological domains, ontological and epistemological stances inform how participant observation is framed, enacted, and drawn upon during analysis (Berthelsen et al., 2017). The constructivist lens of CGT urges the observer to become part of the world being observed (Berthelsen et al., 2017; McCurdy \& Uldam, 2014). As such, effort was made to engage in moderate or active participation in each setting where appropriate.

At the same time, it is acknowledged that a researcher's role can be dynamic, as levels of active engagement shift over time and across places within the course of study (McCurdy \& Uldam, 2014). For example, as part of this study I attended a lobbying and rally event hosted by a group of environmental activists. My early encounters at the event were as quiet and passive observer. However, as energy built through song and protest, it became increasingly awkward to sit by without more active participation. Thus, my role evolved to align more closely with the individuals I was observing and I joined in their chants and rallying cries for a healthier environment. Through this, I came to better understand the courage and confidence necessary to take bold actions in a setting where stakes are high, and to bear witness to the importance of youth having a voice in these matters. In this regard, caution was exercised in each setting such that I did not impede the activity, voices, or emotional safety of the participants and individuals I was observing (Case, 2017).

\section{Field notes.}

Field notes were taken both during the act of participant observation and immediately following my interactions in these settings. The content of field notes 
aimed for descriptive details to be recorded relating to the space, actors, activities (of groups), objects, acts (of individuals), events, time, objectives, and feelings (Creswell \& Poth, 2017; Spradley, 1980). Therein, goals included to avoid inferences and assumptions (e.g., assuming two people are a couple), and to make note of my actions, feelings, and thoughts during the participation through reflective writing as well (Creswell \& Poth, 2017; Lofland \& Lofland, 1995; Padgett, 2008). Insights revealed through informal conversations with actors in the space were also recorded in field notes. Table 2 provides an example of both descriptive and reflective field notes using a template provided by Creswell and Poth (2017). These were recorded during a community clean-up event which is referenced in the following chapter.

Table 2. Field Notes Example from Community Clean-Up.

\begin{tabular}{|c|c|}
\hline Descriptive & Reflective \\
\hline $\begin{array}{l}\text { I arrived at the kick off prior to the } \\
\text { clean-up event. The local coffee shop } \\
\text { was full of young adults drinking } \\
\text { specialty coffees and breakfast items } \\
\text { like avocado toast. After waiting a } \\
\text { while I could not identify who was } \\
\text { organizing the event or if there was a } \\
\text { place to sign up or check-in. } \\
\text { Eventually, a couple of older adults } \\
\text { next to me asked if I knew anything } \\
\text { about the clean-up event. They had } \\
\text { recently relocated to the community } \\
\text { after retirement. Eventually, I made my } \\
\text { way around the coffee shop asking } \\
\text { different individuals if they were } \\
\text { involved in the clean-up. Finally, I } \\
\text { located the young men who were } \\
\text { organizing the event and they provided } \\
\text { me with the details of where to meet } \\
\text { and receive supplies once we arrived at } \\
\text { the location about } 20 \text { miles away. }\end{array}$ & $\begin{array}{l}\text { My immediate reaction to this situation was } \\
\text { one of frustration and anxiety. I, like the } \\
\text { older adults next to me, craved more } \\
\text { organization and leadership demonstrated in } \\
\text { the event. I was expecting someone to stand } \\
\text { up, introduce themselves, thank folks for } \\
\text { coming, and give us the details we needed. I } \\
\text { was curious if this was a demonstration of } \\
\text { good intentions but lack of preparation to } \\
\text { articulate plans and actions. Everyone found } \\
\text { out about the event through Facebook, but } \\
\text { maybe the social media platform was where } \\
\text { the organizer's engagement with potential } \\
\text { participants ended? Later in the day, my } \\
\text { mother who attended with me explained that } \\
\text { the older adults had admired my initiative to } \\
\text { find out what was going on and had said, } \\
\text { "She's a leader." My mother further } \\
\text { commented that the young people organizing } \\
\text { the event lacked "manners." This scenario } \\
\text { reveals a need to train and working across } \\
\text { platforms and demographics to achieve local } \\
\text { and regional goals. There are clearly big } \\
\text { differences here in the way young people } \\
\text { handled their event and the expectations of } \\
\text { other participants. }\end{array}$ \\
\hline
\end{tabular}




\section{Document review.}

Documents provided a third source of data to examine. I examined extant documents to support and critically evaluate data collected through interviews and participant observation. This added to the rigor of the employed methods and boosted the trustworthiness of findings. Documents were identified by searching organizational and community websites and by following regional organizations and news outlets through Facebook. Documents collected included news articles and video clips, as well as social media postings related to regional organizations and current issues. I also collected publicly available reports from organizations associated with individual research participants or prominent social, economic, or environmental issues. A total of 45 primary documents were collected.

Using the CGT lens, documents were viewed as texts that provide additional insight into the research questions (Charmaz, 2014). This is true in that documents reveal positions and discourses from nuanced perspectives and for varied audiences (Charmaz, 2014). For example, documents assisted in examining the various ways that individuals and groups communicate their identities and clarified formal agendas and goals for their work (La, 1995). Documents were also used to situate data within the larger social, economic, and political context of regional issues.

\section{Data Management}

Once each interview or observation was complete, the recording devices, memos, field notes, situational maps, and information sheets were transported from the site of collection to my residence using a Master Lock File Safe, Locking Document Box. Recordings were transferred to a password protected folder in Dropbox $^{\mathrm{TM}}$ using de-identified file names. Paper data sheets (e.g., memos, field notes) were blacked out and shredded after uploading to Dropbox ${ }^{\mathrm{TM}}$. Only the primary 
researcher had access to data sheets and recordings during the course of the project. All but two of the 45 documents collected were available electronically and links were recorded in a Microsoft Word document along with brief descriptions. The remaining two documents were kept in the Master Lock File Safe, Locking Document Box.

Following secure storage, each interview recording was transcribed. Having others transcribe interviews presents the possibility that researchers lose a sense of closeness to the data (see Padgett, 2008). Acknowledging this, I personally transcribed 33 percent of $(n=10)$ interview recordings. GoTranscript (2018) services were used to complete the remaining interview transcripts $(n=20)$. This service maintains a rigorous commitment to confidentiality, including by such procedures as dividing transcripts among transcriptionists so that no single individual has access to an entire recording (GoTranscript, 2018). In order to maintain closeness to all data, transcripts sent to the service were audited for accuracy (Tuckett, 2005).

De-identified transcripts of each recoding were stored within Dedoose ${ }^{\mathrm{TM}}$, a password protected, web based data analysis program (Sociocultural Research Consultants, LLC, 2014). Such programs assist in the management of large volumes of data and can speed up the process of creating, coding, and sorting extracts and attaching analytic and methodological memos (Silverman \& Marvasti, 2008). It is important to note, however, that Dedoose ${ }^{\mathrm{TM}}$ does not operate from an internal system of logic. Such software requires the insight and direction of the researcher to make sense of the data analytically (Silverman \& Marvasti, 2008).

\section{Data Analysis}

Analysis in CGT is an ongoing process with multiple stages and useful strategies (Charmaz, 2014). This section describes the use of memo writing, coding, 
diagramming, constant comparison, situational analysis, and reflexivity as fundamental techniques used in the interpretative process. While presented linearly, it is important to emphasize that these were conducted iteratively alongside data collection, as the CGT process demands (Charmaz, 2014).

\section{Coding.}

A critical way in which CGT differs from other qualitative methods, is its insistence upon iteratively generating analytic categories versus sorting data based on themes or topics (Charmaz, 2014). In this way, the emphasis is upon constructing a framework that interprets participant experiences, rather that applying existing theory to collected data or simply describing data in an organized fashion (Charmaz, 2014). CGT methodologists often retain the basic coding techniques of GTM to support this aim (e.g., Clark, 2005; Charmaz, 2014). In this study, initial, focused, axial, and theoretical coding strategies were used (Charmaz, 2014; Corbin \& Strauss, 2015). Through each stage, emphasis was placed on participants' actions and meanings using gerunds (Charmaz, 2014).

During initial coding, gerunds were used to code each line of the interview, (Charmaz, 2014). This was conducted for 26 percent $(n=8)$ of interview transcripts. Combined with memo writing, these coding exercises served as the initial grounds for creating focused codes that represented prominent strategies, actions, and processes shared by participants. Focused codes were then applied to all transcripts $(\mathrm{N}=30)$ using Dedoose. ${ }^{\mathrm{TM}}$ After focused codes were applied to the data, memo writing was used to make comparisons from multiple sources in support of these codes, and to compare codes in an effort to describe their relationships to one another (Charmaz, 2014). 
As focused coding progressed, axial coding occurred to establish higher level analytic categories, or codes that subsumed multiple focused codes (Charmaz, 2014; Corbin \& Strauss, 2015). This was facilitated by printing and examining existing codes and excerpts fro Dedoose ${ }^{\mathrm{TM}}$ and then re-organizing them by hand. As these categories were clarified, axial coding helped to identify their properties and dimensions (Charmaz, 2014). Properties are characteristics of the analytic categories derived from the data and which differentiate them from one another (Corbin \& Strauss, 2015). Dimensions refer to the range of values, positions, or characteristics which each of these properties may have (Corbin \& Strauss, 2015). Positional mapping, diagramming, and constant comparison also aided in the identification of properties and dimensions.

Also useful at this stage was using previously identified sensitizing concepts to assist in determining the relationship between various categories developed in the analysis (Charmaz, 2014). These included place based concepts identified in Chapter 2. However, in this process it was critical that these not be applied onto the data; but rather used to assist in the interpretation that is warranted by the data (Charmaz, 2014). Also important in this process was to maintain a focus on the goal to develop a context specific framework relevant to participant experiences, rather than developing an overarching theory that could be generalized beyond this context.

Table 3 provides an example of how coding evolved from the original data in a transcription into various coding strategies. This excerpt occurs within the context of an individual describing the work of her organization. It demonstrates that multiple possible initial codes are initially developed, and these become more analytic and comprehensive as the process moves forward.

Table 3. Illustration of Coding Strategies 


\begin{tabular}{|c|c|c|c|}
\hline $\begin{array}{c}\text { Original } \\
\text { Transcription }\end{array}$ & Initial Coding & $\begin{array}{c}\text { Focused } \\
\text { Coding }\end{array}$ & Axial Coding \\
\hline $\begin{array}{l}\text { We focus on } \\
\text { amplifying voices of } \\
\text { young people in the } \\
\text { region so that elected } \\
\text { representation } \\
\text { actually represents us, } \\
\text { so that we have a say } \\
\text { at the table as } \\
\text { decisions are being } \\
\text { made about what } \\
\text { comes next in this } \\
\text { area. }\end{array}$ & $\begin{array}{l}\text { Amplifying voices; } \\
\text { Targeting youth; } \\
\text { Demanding } \\
\text { representation; } \\
\text { Placing work in } \\
\text { regional context; } \\
\text { Ensuring youth } \\
\text { representation; } \\
\text { Making a place at the } \\
\text { table (for youth); } \\
\text { Involving youth in } \\
\text { future decision making }\end{array}$ & $\begin{array}{l}\text { Connecting } \\
\text { to the Bigger } \\
\text { Picture }\end{array}$ & $\begin{array}{l}\text { Spatial Property: } \\
\text { Regional } \\
\text { Dimension } \\
\text { Temporal } \\
\text { Property: Present } \\
\text { and Future } \\
\text { Dimensions }\end{array}$ \\
\hline
\end{tabular}

\section{Memo writing.}

Memo writing includes the researcher's early responses to the data (Charmaz, 2014; Rubin \& Babbie, 2016; Strauss \& Corbin, 1990) and is at the core of the grounded theory process (Glaser, 1978). Writing memos allows the researcher to record emergent ideas and analytic insights as categories and concepts are developed, re-ordered, abandoned, and revived through the ongoing iterative process of data collection and interpretation (Glaser, 1978). The insights formed during memo writing provoke new directions to pursue during both subsequent data collection activities and assist in moving the inquiry and interpretation from description to theoretical insight (Glaser, 1978). This process was aided by Charmaz's (2014, p. 169) outline of questions to ask of the data, such as "What are people saying or trying to say? What do they remain silent about? What accounts for their silence? When, if at all, do secrets shape what's happening?" Memo writing also captured methodological challenges or pitfalls, such as hesitant participants or poor interviewing, and served as a space to engage reflexively about my own assumptions about the data, or how my own identities or behaviors may have impacted the interview. 
Interviews and observations were followed by memo writing, and memos were formed around groupings of collected documents based on issues covered. These insights and reflections were then organized by participant and attached to individual codes and categories as they developed. This was accomplished using functions of the Dedoose ${ }^{\mathrm{TM}}$ data management software.

\section{Diagramming.}

Diagramming of emerging results is also a useful analytic exercise (Corbin \& Strauss, 2015). As focused codes, categories, properties, and dimensions developed, I illustrated these through drawings. Diagrams aided in conceptualizing the connections and directions between various results. They also provided visual aids that were shared with and edited through individual interviews and member checking exercises in later stages of the research process. Final versions of these diagrams are shared in Chapters Four through Six.

\section{Constant comparison.}

Analysis was also facilitated by the use of the constant comparison method (Corbin \& Strauss, 2015). Through this method, relevant statements were compared across types of data for each code and category leading to further scrutinizing and development (Charmaz, 2014). In addition to contributing to a final interpretive framework, constant comparison assists in understanding where data is thin or missing, and which dimensions and properties can be sought out through subsequent data collection (Rubin \& Babbie, 2016).

\section{Situational analysis.}

Situational analysis was developed by Adele Clarke (2005), as an extension of GTM within a postmodern lens. Whereas GTM analyses emphasize action and processes of key actors, situational analysis incorporates non-human elements such as 
discourses, providing a holistic backdrop to the research endeavor (Clark, 2005).

Clark (2005) identifies three types of maps used in analysis. This study made use of two of these: ordered situational map and positional maps (Clark, 2005).

\section{Ordered situational maps.}

Ordered situational maps identify elements of a situation and interrogate relationships between elements (Clark, 2005). As illustrated in Table 4, these elements may include those of time, space, human and nonhuman actors, discourses, and symbols. The creation of these maps was informed by both data and sensitizing concepts. Situational mapping assisted the developing analysis by providing prompts for questioning the relations between elements identified in the data and to situate participant experiences, observations, and documents within a broader context (Clarke, 2005).

Table 4. Ordered Situational Map (Clarke, 2005, p. 90)

\begin{tabular}{|l|l|l|}
\hline $\begin{array}{l}\text { Individual Human } \\
\text { Elements/Actors }\end{array}$ & Temporal Elements & $\begin{array}{l}\text { Discursive construction of } \\
\text { nonhuman actors/actants }\end{array}$ \\
\hline $\begin{array}{l}\text { Collective Human } \\
\text { Elements/Actors }\end{array}$ & Major Issues/Debates & $\begin{array}{l}\text { Sociocultural/symbolic } \\
\text { elements }\end{array}$ \\
\hline $\begin{array}{l}\text { Discursive Constructions } \\
\text { of Individual and/or } \\
\text { collective human actors }\end{array}$ & $\begin{array}{l}\text { Nonhuman } \\
\text { Elements/Actors }\end{array}$ & Spatial Elements \\
\hline $\begin{array}{l}\text { Political/Economic } \\
\text { Elements }\end{array}$ & $\begin{array}{l}\text { Implicated/Silent } \\
\text { Actors/Actants }\end{array}$ & $\begin{array}{l}\text { Related Discourses } \\
\text { (historical, narrative, and/or } \\
\text { visual) }\end{array}$ \\
\hline Other & \multicolumn{2}{|l}{} \\
\hline
\end{tabular}

\section{Positional maps.}

Positional maps are used to illustrate the varied stances that are taken with regards to issues or discourses that arise within the data and research context (Clark, 2005). Clark (2005) emphasizes that by mapping these positions, analysis can avoid 
an over focus on similarities and highlight silences, differences, and complexities among positions. Clark's (2005) method demands looking at positions themselves. For example, one significant category of results was labeled Connecting to the Bigger Picture (see Chapter 6), as this became a dominant process through which individuals made meaning of their decisions to stay and work on issues within Appalachian Kentucky. Positional maps were used to identify where participants fell along two axes that emerged within this category: (1) a temporal axis reflecting past, present, and future connections and (2) a spatial axis representing their connections to broader contexts ranging from individual to global. The maps created for this category illustrated the various stances taken along these axes with regards to meaning making. A positional map is provided below (Figure 1), illustrating the range of data collected in support of this category.

In addition to using positional maps in this way, this analytic strategy was also used to map individuals (rather than positions or stances). For example, over the course of data collection and early analysis, it became clear that many participants experienced conflicts in their personal and professional relationships as a result of real and perceived differences. As a result, I began to map individuals along a continuum of low to high levels of conflict based on my perception of what they revealed in their interviews. In doing so, I was able to make important comparisons between participants as well as to follow up with these participants as to whether my placement of their experience felt accurate and to hear more about the role conflict played in their experience. 


\begin{tabular}{c|ccccc} 
Global & $\mathrm{x}$ & $\mathrm{x}$ & $\mathrm{x}$ & $\mathrm{x}$ & $\mathrm{x}$ \\
National & $\mathrm{x}$ & $\mathrm{x}$ & $\mathrm{x}$ & $\mathrm{x}$ & $\mathrm{x}$ \\
Regional & $\mathrm{x}$ & $\mathrm{x}$ & $\mathrm{x}$ & $\mathrm{x}$ & $\mathrm{x}$ \\
State & $\mathrm{x}$ & $\mathrm{x}$ & $\mathrm{x}$ & $\mathrm{x}$ & $\mathrm{x}$ \\
Local & $\mathrm{x}$ & $\mathrm{x}$ & $\mathrm{x}$ & $\mathrm{x}$ & $\mathrm{x}$ \\
Family & $\mathrm{x}$ & $\mathrm{x}$ & $\mathrm{x}$ & $\mathrm{x}$ & \\
Individual & $\mathrm{x}$ & $\mathrm{x}$ & $\mathrm{x}$ &
\end{tabular}

Distant Past Recent Past Present Near Future Distant Future

\section{Figure 1: Connecting to the Big Picture: A Positional Map}

\section{Sufficiency, saturation, and theoretical sampling.}

There are no hard and fast rules regarding sample size in qualitative studies (Padgett, 2008). Emphasis is placed on depth, rather than the size of the sample (Padgett, 2008). The goal of collecting data is to reach sufficiency and saturation, and these factors determine sample size. Sufficiency refers to the data's ability to illustrate depth and breadth of the topic, including that the data collected: covers the views of a range of participants, includes multiple actions and processes among each participant, and reveals processes occurring below the surface level enabling the development of analytic categories (Charmaz, 2014). In CGT, saturation occurs when the dimensions and properties of the analytic categories can all be supported by data with rich details, and the working theory or framework can be developed (Charmaz, 2014). In grounded theory, this is typically achieved at between 20 and 30 participants (Creswell \& Poth, 2018; Padgett, 2008).

Examination of sufficiency and saturation was facilitated by coding, positional mapping, diagramming, and constant comparison. Where gaps were identified, 
theoretical sampling was used to identify new sources of data to provide further insight or to fill in dimensions and properties (Rubin \& Babbie, 2016). For example, positional mapping revealed that most participants made meaning of their work by looking at its impact in the present or the foreseeable future. This process was coded as reflecting across time. However, in order to achieve sufficiency and saturation in this code, additional data was needed from participants who made meaning of their work by drawing on the past. To collect this data, I recruited two additional participants who had worked with organizations or in positions where history was explicitly used or explored in relation to social, economic, and environmental issues. I also gave existing participants the opportunity to reflect on the past during follow-up stages.

In this way, theoretical sampling occurred both with new participants and during member checking (see below), by asking previous participants to clarify or go in-depth about a previously discussed topic (Charmaz, 2014). Interviews conducted in this pursuit were recorded and managed in a parallel fashion to the methods described above. Where existing participants were asked follow up questions, select excerpts were transcribed and coded by myself as needed. Theoretical sampling was determined to be complete when the context specific framework being developed was saturated with material that filled the categories, properties, and dimensions, and when relationships between categories were clarified (Charmaz, 2014; Glaser, 1978).

\section{Reflexivity}

In qualitative research, the meaning of reflexivity is to turn back on oneself to illuminate how one's own values, positions, personal histories, identities, biases, privileges, and disadvantages influence the research and those involved (Finlay, 2002; Hammersley \& Atkinson, 2007; Luttrell, 2000). Reflexivity thus demands personal 
transparency and openness regarding the social and interactive nature of the research process (Hammersley \&Atkinson, 2007). Instead of eliminating the researcher's influence upon participants and activities, reflexive inquiry insists that the role of the inquirer is to understand those interactions and their meanings (Hammersley \& Atkinson, 2007). These subjectivities then become part of the data observed, recorded, and analyzed (Berthelsen et al., 2017; Case, 2017; Charmaz, 2017; Ellis \& Flaherty, 1992; McCurdy \& Uldam, 2014; Padgett, 2008). Reflexivity is thus a tool for the examination of "ethically important moments" (Case, 2017, p. 398; see also Gildersleeve, 2010; Guillemin \& Gillam, 2004) and the production of "emotionally sensed knowledge" (Evans et al., 2017, p. 585).

I practiced reflexivity within each stage of the research process. Through the writing of memos and field notes, I collected and interpreted data related to my myriad subjectivities and their potential impact on participants, settings, and ultimately the analysis itself. Among the most significant themes that emerged in this process was ongoing tension between my insider and outsider statuses. Though sometimes neatly compartmentalized, the pros and cons of the insider/outsider dilemma can be over generalized (Styles, 1979). In fact, it has been argued that the binary insider/outsider debate itself is what is truly problematic (Hammersley \& Atkinson, 2007; see also Larabee, 2002; Merton 1972; Kusow, 2003). Rather, as McCurdy and Uldam (2014) note, both insider and outsider statuses are occupied along a continuum. Additionally, our positions with respect to this continuum shifts with each interaction among research participants, settings, and data (McCurdy \& Uldam, 2014). In this way, I occupied both insider and outsider statuses in relation to various issues, settings, and participants encountered in this study. 
As a Millennial, a native of Appalachian Kentucky, and a social worker who formerly worked in the region, I consider myself an insider within the research context. Moreover, as an individual who experienced struggles related to my political, religious, and sexual identity in place, I felt a closeness with participants who shared these challenges. These subjectivities offered a number of methodological and analytic benefits. I enjoyed knowledge of the issues under study, organic entry into certain social settings, and ease of access and rapport building with many participants (Adler \& Adler, 1994; Bonner \& Tolhurst, 2002; Hodkinson, 2005; Juris, 2007; McCurdy \& Uldam, 2014; Padgett, 2008; Pugh, Mitchell, \& Brook, 2000; Watts, 2011). At the same time, insider status posed multiple challenges. For example, insiders run the risk of overlooking certain patterns and routines and may over identity with participants (Bonner \& Tolhurst, 2002; Chen, 2011; Gerrish, 1997; Lipson, 1984; Padgett, 2008; Timseena, 2009). Thus, the opportunities of insider status had to be balanced with maintaining analytic distance (Atkinsen and Hammersley, 1994; Bonner \& Tolhurst, 2002; Hammersley \& Atkinson, 2007; Spradley, 1990).

At the same time that I honored my insider status, I also recognized the many ways in which I acquired outsider status. For instance, through my own life decisions and circumstances, I was unable to stay long term in the region and commit to the kinds of work the participants in this study were doing therein. Moreover, I was not a member of many of the communities and organizations where data was collected and there were significant areas of participant experience I did not share as a white, cisgender female doctoral candidate married to a similarly situated cisgender man. Similarly, there were limits to my ability to relate to participants who did not share my political and social positions on various issues. 
In order to weigh the advantages and disadvantages of these statuses, I continuously identified and wrestled with my political, social, and cultural sympathies. These were processed reflexively through memo writing, peer-debriefing, and member checking (see below). Additionally, positional mapping and theoretical sampling aided in identifying my tendency to emphasize participants who shared my views and experiences. Subsequently, significant efforts were made in sampling, analysis, and writing to include the voices of participants from a wide range of perspectives and to privilege all participant positions over my own (Hammer et al., 2017; Vargas-Cetine, 2013).

\section{Trustworthiness}

Trustworthiness refers to the elements of rigor in qualitative research (Amankwaa, 2016). According to Lincoln and Guba (1985), the trustworthiness of a qualitative study is comprised of the following factors: credibility, transferability, dependability, and confirmability. Credibility refers to confidence in the accuracy and fairness, or 'truth' of qualitative research findings (Amankwaa, 2016; Lincoln \& Guba, 1985). Transferability in qualitative research refers to a study's ability to demonstrate application to other research contexts, populations, or similar issues (Amankwaa, 2016; Lincoln \& Guba, 1985; Padgett, 2008). The dependability of a qualitative study is based upon the consistency of its methods and the ability for these to be audited and repeated (Amankwaa, 2016; Lincoln \& Guba, 1985). Confirmability denotes the importance of shaping results based on participant experience or being explicit where the researcher's subjectivities are incorporated (Amankwaa, 2016; Lincoln \& Guba, 1985).

Challenges to trustworthiness include reactivity (e.g., the researcher distorting participant responses or behaviors), respondent bias (e.g., withholding), and 
researcher bias (e.g., selecting participants who share a similar worldview, distorting interpretations; Padgett, 2008). Trustworthiness is also compromised by lack of organization and record keeping (Koch, 2006). Numerous methods have been supported to decrease the impacts of these risks including triangulation, member checking, peer de-briefing, prolonged engagement, maintaining a decision trail, and providing generous context alongside results (Padgett, 2008). This study draws on each of these strategies as described below.

\section{Triangulation}

Many types of triangulation exist in support of credibility (Amankwaa, 2016; Lincoln \& Guba, 1985; Padgett, 2008). In this study, triangulation of multiple data sources was used for interpretive corroboration. Interview and observational data were cross checked, both with one another and alongside existing literature, media, and collected documents (Rubin \& Babbie, 2016).

\section{Member Checking}

Member checking involves consulting with participants as the analysis develops (Lincoln \& Guba, 1985). This strategy supports trustworthiness and is consistent with a perspective that honors the co-constructed nature of the research process (Birt et al., 2016; Padgett, 2008). In this study, member checking involved consulting with participants regarding their initial interview transcripts, emergent analytic categories, and written results (Birt et al., 2016). These strategies occurred by phone $(\mathrm{n}=5)$ and in person $(\mathrm{n}=11)$. Additionally, three participants were contacted by email and asked to review and approve of case exemplars using their stories. Member checking was conducted with participants from a range of positions within the analytic categories developed. These follow up procedures were used to verify and extend results and identify inconsistencies and biases (Birt et al., 2016; Rubin \& 
Babbie, 2016; Hoover \& Morrow, 2015). Throughout the process, analytic techniques such as reflexivity and memo writing were continued and it was paramount that I remained open to new insights and changes required within the final results as a result of participant feedback (Kornbluh, 2015). In addition to aiding in trustworthiness, member checking provided opportunity for closure with participants as I left the field.

\section{Peer De-briefing}

Peer de-briefing or consultation is important in that it offers multiple perspectives on the data in support of credibility and lends to dependability through oversight (Lincoln \& Guba, 1985; Padgett, 2008). Peer de-briefing occurred with doctoral student colleagues and members of my dissertation committee. In this review process, data collection and analysis strategies were reviewed to insure they were explicit and that results were sustained by the data (Amankwaa, 2016; Lincoln \& Guba, 1985)

\section{Prolonged Engagement}

Prolonged engagement with a population or site of study addresses respondent bias, as withheld or misleading narratives simply don't hold up over time (Amankwaa, 2016; Lincoln \& Guba, 1985; Padgett, 2008). Prolonged engagement was accomplished through repeated participant observation within a single community and a single organization. Prolonged engagement with individual participants occurred through individual interviews, check-in calls and emails, member checking interviews, and sharing of results.

\section{Maintaining a Decision Trail}

Leaving a clear decision trail behind supports oversight and the potential for reproducibility (Koch, 2006; Lincoln \& Guba, 1985; Padgett, 2008; Schwandt \& Halpern, 1988). This study accomplishes this through comprehensive data collection 
including audio recordings and near verbatim transcripts for each individual interview and member checking consultation (Tuckett, 2005). Dedoose ${ }^{\mathrm{TM}}$ software aided in the organization of data and analytic consistency and (Silverman \& Marvasti, 2008). The creation and sharing of coded transcripts, memos, diagrams, and situational maps also aid trustworthiness, as this outlines the evolution of raw data to an interpretive framework (Ruin \& Babbie, 2016). Reflexive practice and documentation also provided methodological and analytic auditability (Lincoln \& Guba, 1985; Luttrell, 2000).

\section{Providing Context}

The importance of providing sufficient context is related to the concern for transferability (Koch, 2006). Generous context about the research environment and methods assists readers in discerning the degree to which one study may be similar to another (Guba \& Lincoln, 1989; Koch, 2006). Depth of context also assists readers interested in similar but not identical situations (Lincoln \& Guba, 1985; Rubin \& Babbie, 2016). This concern is attended to by providing an overview of the Appalachian context and issues of outmigration in earlier chapters, such that readers can understand both what is unique but also shared between this research environment and others. I also elaborate herein on contexts related to my methodological decision making, and those that are necessary for framing the results and subsequent discussion.

\section{Writing and Dissemination}

Writing in qualitative research does not exist in isolation from the data collection and analysis (Silverman \& Marvasti, 2008). Writing too, is part of the development of the results (Glaser, 1978). Whereas previous sections have included 
examples of writing as part of data collection and analysis, this section emphasizes issues encountered in the final writing stages and through dissemination of results. Paramount in this stage were concerns regarding the ethical inclusion of self in the final report, representation of the participants and issues of study, and reciprocity between myself and the participants.

\section{Inclusion of Self}

While reflexivity was a key analytic tool in this study, decisions needed to be made with regards to how my voice and positions would be included in this report (Padgett, 2008; Soobryan, 2003). My approach is to include first person voice in this chapter, to lift up the situated nature of the knowledge I gathered and my own role in the interpretive process (Aagard \& Matthiesen, 2016; Haraway, 1988; Højholt \& Kousholt, 2014). In later chapters, the voices of participants are given center stage and I include myself where necessary to clarify how I may have impacted results (Rubin \& Rubin, 2012; Vargas-Cetina, 2013). Through this, I aimed to maintain a balance between my role in this analysis and lifting up the voices of the participants in the study and their experiences.

\section{Representation}

Scholars began to write of the "crisis of representation" in the 1980s (e.g., Clifford 1986a, 1986b; Marcus \& Fischer, 1986). This crisis referred to growing criticism of the ways in which researchers speak of and for various people groups who may not wish or need for them to do so (Vargas-Cetina, 2013). Recognizing these debates, I admit the product of this inquiry is yet another form of representation of Appalachian people and places, and as such it no less political in nature than previous accounts of the region (Haynes, 1997; Vargas-Cetina, 2013). Like popular stereotypes, scholarly depictions have the potential for 'othering' Appalachians and 
placing blame for problems on individuals that are often structural in nature (Haynes, 1997). This has also been true for Millennials at large (e.g., Bauerlein, 2008; Stein, 2013; Twenge \& Campbell, 2010). Therefore, as part of the ethical concern for the population studied, it was critical that their experiences be shared with generous context and that voices of the participants themselves be prioritized during analysis and writing. Through this, I also make efforts to avoid over generalizations of the sample and to provide ample details regarding the differences among participants in each category of results.

\section{Reciprocity}

Researchers often experience tension between their role as academic and the responsibility they feel to make something meaningful of their research in the eyes of participants (Luttrell, 2000). Issues of power and reciprocity arose during the writing and dissemination phase, in that ultimately the rewards I gained are much more than what one receives as a participant (Seidman, 1991). My approach to this has been to demonstrate transparency with participants throughout my process, and by sharing copies of the final product with each participant (Rubin \& Babbie, 2016; Seidman, 1991). This is a commonly neglected form of payback to such contributors and a necessary component of maintaining goodwill and rapport (Padgett, 2008; Wolcott, 2001). Additionally, I plan to accept invitations to discuss and share this work with interested participants and organizations, as well as to further disseminate findings through publication.

\section{Summary}

This chapter provides an overview of the CGT approach and details the methodological strategies used to collect, analyze, and report the results that appear in following chapters. Overall, the aim of this approach has been to analyze patterns and 
processes in the creation of an interpretive framework that encompasses the most significant concepts revealed within the data (Padgett, 2008). Thus, while not all participant experiences are reflected in this account, the following chapter explores the most prominent processes emerging within participants' shared experiences. 


\section{CHAPTER FOUR: RESULTS}

Three questions were posed at the start of this research: 1) What processes inform young adults' decisions to live and work in Appalachian Kentucky? 2) What strategies do young adults engage to maintain these commitments? and 3) How do young adults make meaning of these commitments? By implementing the methods discussed in the previous chapter, analytic categories were developed in alignment with each question, respectively: 1) working out place, 2) keeping peace in place, and 3) connecting to the big picture. Each of these questions was investigated in service of the two overall aims established for this study. The first aim was to understand and describe young adults' commitments to staying and working in Appalachian Kentucky as well as the meaning they make of these decisions. The second was to develop a context specific framework that would facilitate interpretation of these decisions and processes.

Following a description of the interview sample, this chapter fulfills the first aim by explicating three analytic categories which align with the proposed research questions. Each of these categories offers description of the conditions under which each occurs and how they are experienced from various positions among participants and as noted through observation and document review. Throughout these sections, participant quotes support the analytic descriptions and illustrate how these processes transpire for individuals. Effort was made to include the voices of different participants in the study; highlighting excerpts where they spoke with the most clarity and insight regarding a particular topic. 
Moreover, each section concludes with a case exemplar, chosen for its ability to demonstrate each of the relevant processes being examined with rich detail. While all cases offered descriptions and insight into some or even most of the analytic processes that were developed, the individuals chosen for the case exemplars eloquently articulated each task within the categories. The chapter concludes by satisfying the second study aim. This is achieved with a description of doing place; a context specific framework that encompasses the relationships between the three analytic categories and expands the overall interpretation of young adults' commitments to Appalachian Kentucky.

\section{Sample Description}

Interview participants were recruited for this study based on five sampling criteria. These included that they: (1) currently lived in Appalachian Kentucky; (2) had lived in the region least 15 years (cumulatively); (3) were born between the years 1980 and 2000; (4) had completed or attained some college education; and (5) were working on social, economic, and environmental issues within the region. In addition to these criteria, data were collected regarding each participant's gender, race, and their areas of work. Table 5 compiles this data. This is accompanied by a description of each demographic category below.

\section{Age, Race, and Gender}

Participants interviewed for this study $(\mathrm{N}=30)$ ranged in age from 18 to 38 years old. The sample average was 29 years of age at the time of interview. Age is also reported by the following groupings: $18-21(n=5), 22-25(n=4), 26-30(n=8), 31-$ $34(n=4)$ and 35-38 $(n=9)$ years. Race and gender are reported according to the language individuals shared with the interviewer. The IRB approved collection of race and gender data as a later amendment and thus the majority of it was received 
during follow up contacts. During this process, four participants did not respond with the requested information. Of the remaining 26 participants, 1 identified as African American and 25 identified as White or Caucasian. Thirteen participants identified as female, 1 as Cisgender Female, 10 as male, 1 as non-binary transgender, and 1 as gender queer.

Table 5. Demographic Data for Interview Participants

\begin{tabular}{|c|c|}
\hline Data Category & $\begin{array}{l}\text { Results } \\
(\mathbf{N}=30) \\
\# /(\%)\end{array}$ \\
\hline $\begin{array}{l}\text { Age } \\
18-21 \\
22-25 \\
26-30 \\
31-34 \\
35-38\end{array}$ & $\begin{array}{l}5(16.6) \\
4(13.3) \\
8(26.6) \\
4(13.3) \\
9(30)\end{array}$ \\
\hline $\begin{array}{l}\text { Race } \\
\text { White/Caucasian } \\
\text { African American } \\
\text { No response }\end{array}$ & $\begin{array}{l}25(83.3) \\
1(3.3) \\
4(13.3) \\
\end{array}$ \\
\hline $\begin{array}{l}\text { Gender } \\
\text { Female } \\
\text { Female Cisgender } \\
\text { Male } \\
\text { Gender Queer } \\
\text { Non-binary Transgender } \\
\text { No response }\end{array}$ & $\begin{aligned} & 13(43.3) \\
& 1(3.3) \\
& 10(33.3) \\
& 1(3.3) \\
& 1(3.3) \\
& 4(13.3) \\
&\end{aligned}$ \\
\hline $\begin{array}{l}\text { Education (by highest level completed) } \\
\text { Less than Bachelor's } \\
\text { Bachelor's } \\
\text { Master's } \\
\text { Doctorate }\end{array}$ & $\begin{array}{c}6(20) \\
10(33.3) \\
10(33.6) \\
4(13.3) \\
\end{array}$ \\
\hline $\begin{array}{l}\text { Employment Status } \\
\text { Full-time } \\
\text { Part-time } \\
\text { Unpaid Volunteer }\end{array}$ & $\begin{array}{c}24(80) \\
1(3.3) \\
5(16.6)\end{array}$ \\
\hline $\begin{array}{l}\text { Types of Engagement } \\
\text { Education \& Youth Development } \\
\text { Arts \& Media } \\
\text { Environmental } \\
\text { Activism \& Advocacy } \\
\text { Economic } \\
\text { Social Services }\end{array}$ & $\begin{array}{c}11(36.6) \\
9(30) \\
9(30) \\
4(13.3) \\
13(43.3) \\
11(36.6) \\
\end{array}$ \\
\hline
\end{tabular}




\section{Education and Work}

All participants in this study had completed some college education at the time of interview and were working on social, economic, or environmental issues within Appalachian Kentucky. Six participants had begun but not completed their Bachelor's degree. Of these, five were currently enrolled as full-time students. Ten participants had earned their bachelor's degree by the time of their initial interview while another 10 had completed at least one master's level degree. Four participants had completed doctorate degrees in their fields. With regards to employment, twenty-four participants were working full-time and one participant worked part-time. The remaining five participants were full-time students engaged in part-time volunteer commitments.

Most individuals were involved in multiple organizations and activities, including those that crossed the domains of social, economic, and environmental issues. For example, individuals engaged in agriculture spoke of the importance of this to both environmental and economic concerns within the region. Many of these individuals were also involved in other paid employment to supplement farm income, as well as volunteered or were members of other social and civic groups. Similarly, entrepreneurs saw connections between their work and tourism, as well as the overall economic development of the region; but also felt they had a role in changing the social climate of their communities and the region at large. It is therefore difficult to capture the full breadth of involvement and effort put forth by the participants in this study by capturing a single category of their engagement. Thus, the numbers presented here reflect that most individuals were committed to more than one activity or their activity crossed these domains. Included in these numbers are both the individual's primary place of employment or of significant unpaid efforts, and the 
domains of engagement with which they spoke during the interviews. These are described below.

Education and youth development. This category includes participants $(\mathrm{n}=11)$ who worked directly with students within schools or through programming and organizations serving children and youth outside of the school setting.

Arts \& media. Participants $(n=9)$ here are engaged in both paid and unpaid activity related to the digital arts and marketing, as well as all visual and performing arts activities. This includes artists working directly with a medium and those in administrative roles.

Environmental. This category encompasses all the individuals $(\mathrm{n}=8)$ within the study whose full or part time efforts involve a significant commitment to the environmental health of the region. This includes activism, natural resource management (e.g. forestry), agriculture, and sustainability efforts (e.g. recycling, renewable energy projects).

Activism and advocacy. Included here are individuals $(\mathrm{n}=4)$ who spent a significant amount of time organizing and participating in rallies, protests, and demonstrations; as well as hosting educational or informational events designed to motivate social and political change in areas other than environmental.

Economic. Economic endeavors included direct development projects such as job creation, downtown revitalization, tourism, and entrepreneurship. Activities in this realm were undertaken by many of the participants $(n=13)$; either as their primary undertaking or in addition to their other work.

Social services. Finally, several participants $(n=11)$ were engaged in direct social service provision in areas such as substance use counseling, basic needs assistance (e.g. housing, clothing, and food provision), domestic violence prevention, 
adult education, and legal services. Also included in this category are those individuals who do not directly provide these services but who serve in administrative or support roles within such programs or systems.

\section{County of Residence}

Participants were recruited from 18 of the 54 Appalachian Counties in Kentucky. While all participants currently worked and lived within the region, some worked in counties adjacent to those where they lived. Similarly, while all college students were attending a school within the region, they each also had different Appalachian counties of permanent residence. Here, participants are reported by their county of residence, including the county of permanent residence for full-time students living on campus. Figure 2 illustrates the counties of residence represented in the sample. These included: Bell $(n=1)$, Clay $(n=2)$, Cumberland $(n=1)$, Green $(n=1)$, Greenup (n=1), Harlan ( $\mathrm{n}=6)$, Jackson $(\mathrm{n}=1)$, Knox $(\mathrm{n}=2)$, Laurel $(\mathrm{n}=1)$, Lincoln $(\mathrm{n}=1)$, Madison $(\mathrm{n}=3)$, Perry $(\mathrm{n}=1)$, Powell $(\mathrm{n}=1)$, Pulaski $(\mathrm{n}=1)$, Rowan $(\mathrm{n}=1)$, Russell ( $\mathrm{n}=1)$, Whitley $(\mathrm{n}=4)$, and Wolfe $(\mathrm{n}=1)$ Counties.

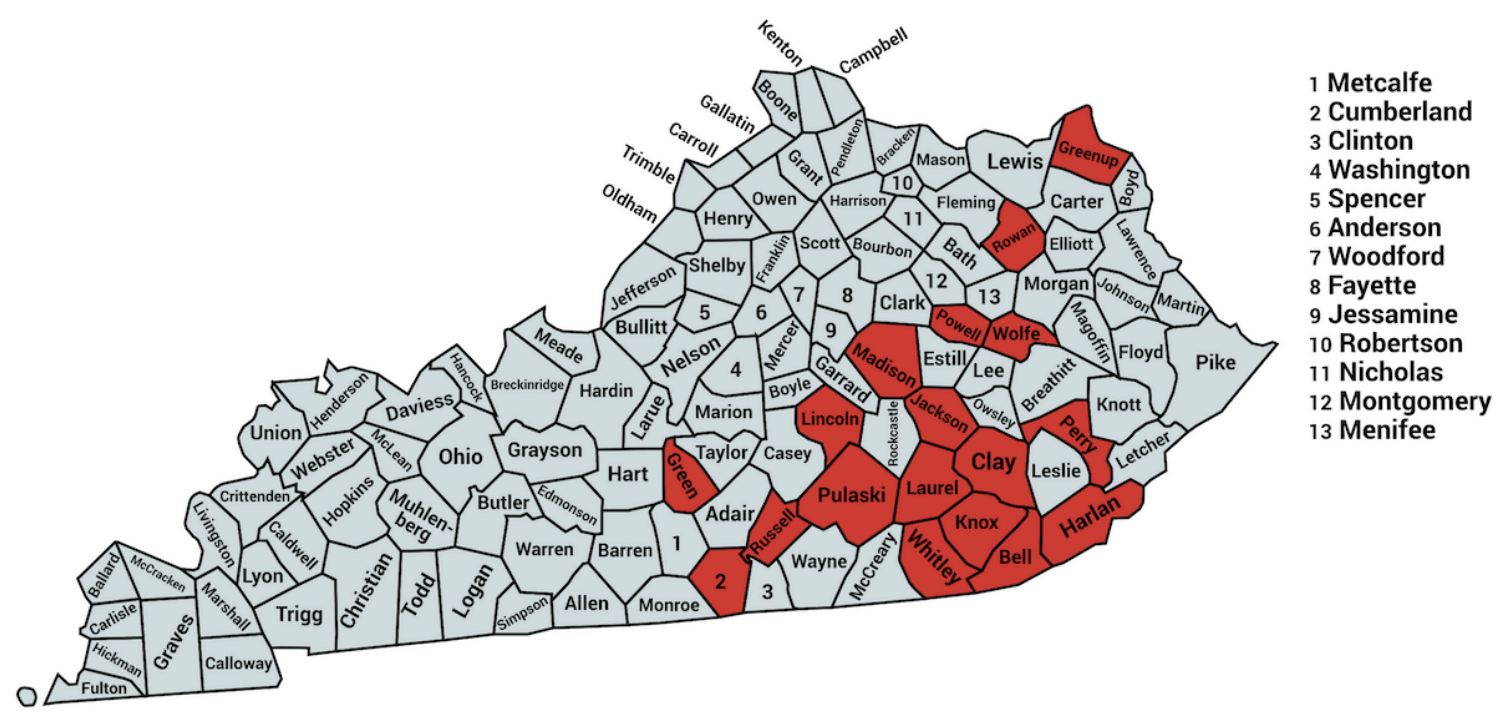

Figure 2. Participants by County of Residence. Created with mapchart.net (C) 
The most common reason for spending time outside the region was for educational opportunities. For undergraduate education, 16 participants attended or were currently attending schools within Appalachian Kentucky. Ten participants attended schools within Kentucky but outside of the Appalachian region and 4 individuals attended schools out of state. Among those who earned graduate degrees $(\mathrm{n}=14), 4$ attended schools within the region or online while living in the region. Five of these individuals attended graduate schools in Kentucky, and 5 went out of state. Among those who attended school out of the region, many stayed in their college communities before moving back to the region. Two individuals spent more than a year working outside Kentucky and two spent a year or more working or volunteering outside the United States.

\section{Category 1: Working out Place}

This inquiry began with the question: What processes guide young adults' commitments to stay and work in Appalachian Kentucky? The aim was to identify how young adults navigate these decisions, regardless of their life course position or their ultimate choice regarding where to live or what to do. Working out place was developed as a descriptive category that encompasses the diversity observed across this decision making process.

Within this category, three distinct phases were identified: (1) getting place, (2) eyeing the door, and (3) securing place. The first and third phase titles were developed by the researcher to represent multiple voices and perspectives. Eyeing the door was an in-vivo quote lifted directly from an interview transcript, which also encompassed varied participant experiences. By laboring through these phases, individuals worked out their unique understanding of their home communities and the Appalachian region. They also developed a sense of their own identities, goals, and 
positions and negotiated these in relation to place. Figure 3 illustrates these stages and identifies major tasks associated with each. Below, these are described in detail with each task underlined in the narrative. This is followed by a case exemplar.

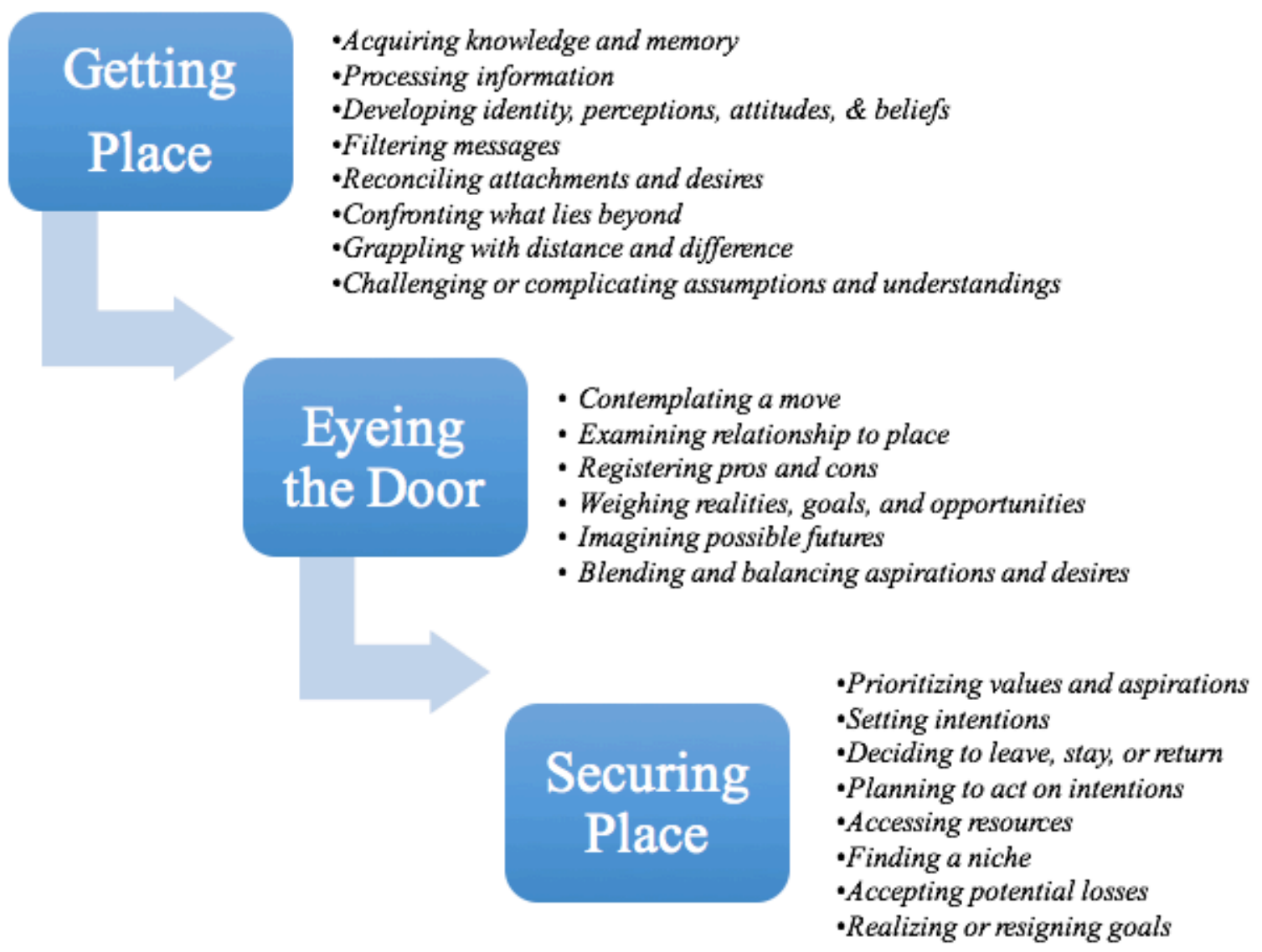

Figure 3. Working Out Place.

\section{Getting Place}

Getting place comprises how young adults came to know and understand Appalachian Kentucky. Early in life, young adults began acquiring knowledge and memories of place; both their home community and the region at large. Processing $\underline{\text { this information then informed their developing identities, perceptions, attitudes, and }}$

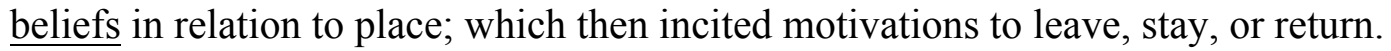
This section will describe how getting place occurs as one is coming of age, but also evolves continuously as individuals renegotiate their relationships with place. 
Through the getting place process, young adults asked questions such as: What is unique about this place? What does it mean to be from this place? Do I belong here? What can this place offer me? What can I contribute here? Will I miss this place if I leave? Is there somewhere, or someone I can come back to if I return? For each individual, a unique set of answers developed over time, based on how they received, interpreted, and synthesized information about their communities and the region; but also about themselves.

This place based knowledge was created and transmitted through a variety of sources and mediums including direct interactions with significant adults, sensory experiences, implied cultural norms, and stereotypes presented by the broader society. Many young adults, for example, developed positive attitudes toward Appalachian Kentucky through formative experiences in the mountains or on farms, art or cultural activities, and by way of the strong social and kinship networks that surrounded them. These encounters then shaped cherished memories of place and nurtured awareness of, as one participated stated, "where and who we're from."

At the same time, most filtered these messages alongside a collection of negative ideas. For example, like many others, Ash's early encounters conveyed a sense that future success required distancing oneself from the region, not only geographically but also culturally, hearing "This place is bad. This place is inferior. Don't talk like these people. Don't do these behaviors if you want to have a decent life... You have to become more than this place if you want to survive." During participant observation at a regional event, I witnessed such messaging firsthand. Sitting among other attendees at a round conference table, we were asked to discuss solutions to regional poverty and other disparities. A state politician sitting at our table responded, "We have to come up with ways for kids to get out." 
As a result of these mixed impressions, getting place for most young adults encompassed reconciling attachments and desires regarding who and where they wanted to be. But in addition to the slow moving accumulation of messages, getting place was also facilitated by single experiences. In Russell's case, this occurred when the landscape of his childhood was destroyed by a pipeline project. He remembered:

Everything I could see was changed. The little rick ledges that used to be there...no more. The trees were gone. The animals were gone. The tranquility was gone... It was all blasted out. Now it's just a grassy little area. It's not even the same place to me anymore. That one event really triggered everything that happened afterwards...[Now], I could never fully leave this area or the work that I do. I think it's important enough to this community, to this area, to stay.

Thus for Russell, one devastating event shaped how he got place as a threatened location. His experience also illustrates how one's concept of place eventually informs their commitments, as the destruction he witnessed sparked his environmental activism within the region.

While getting place includes information about one's home community and the region at large, this understanding also emerges by confronting what lies beyond. For instance, many shared that new appreciations of place developed while they were away from the region, particularly as a result of homesickness and longing. Others expressed that stereotypes and outsider perspectives challenged them to reconnect with home in new ways. In the excerpt below, Becca shares how her perspective on place was altered while attending in graduate school out of state:

I became a little more defensive of where I'm from while I was away. It's like with your family; only family can talk bad about your family...That's how I felt about Appalachia. I was doing some research on the region and people were so curious that I was from Appalachia. They were like, "You know those people?" [One time] I was asked, 'How did you get out?' ...like I needed to escape. That struck a nerve with me. When people would say things like that I would say "Yes, I am those people. I'm proud of where I'm from." Because I was in graduate school, I was able to look at it from a more of a macro level and to understand why some of the problems exist in the region; things that I wasn't really thinking about before. 
For Becca, getting place was facilitated by grappling with distance and difference, but also through education. The salience of getting place in this way was shared by many participants; particularly those who looked closer at the historical and structural conditions that shaped the Appalachian region. This acquisition of higher learning thus facilitated another important task in challenging or complicating their assumptions and understandings of place. In addition to real time experiences and understandings, new insights and reflections and attitudes are generated through nostalgia and memory when one is outside of place.

\section{Eyeing the Door}

A second component to working out place is the process of eyeing the door. Where getting place is situated around comprehending and evaluating place, eyeing the door involves contemplating a move by examining one's relationship to place. Based on a growing understanding of both self and the region of Appalachian Kentucky; each individual began registering the pros and cons of staying or returning. This involved weighing current realities, goals, and opportunities and also imagining possible futures; both in and outside of the region.

Often, eyeing the door emerged as a focused deliberation that preceded making a commitment to a particular place or position. Major life transitions regularly provoked such introspection among participants and intensified this process including through graduations, personal setbacks, the ending or loss of opportunities, and profound moments in close relationships including separations, births, illnesses, and deaths. At others times, eyeing the door was a passive or casual exercise, one which did not precede real intentions of either coming or going. Mike, for example, had no real intentions of leaving his rural hometown since he settled there with his wife and children. However, he still engaged in eyeing the door when thinking about what he 
has gained and potentially sacrificed through his decision. For example, he considered such factors as cost of city rent versus a rural mortgage, as well as his daily commute time. He also considered less obvious factors like the availability of culturally diverse foods in local markets and restaurants, observing, "You can't go and buy just whatever you want at the local grocery store. At the same time, I can grow whatever I want to in my garden. If I want hummus, I'll raise chickpeas."

Similar to Mike, many participants eye the door elsewhere by weighing the pros and cons of one's commitments; both real and imagined. Those who stayed in or returned to rural communities, often envisioned living in cities. While factors like cost of living, traffic, and noise were voiced as deterrents, many also admitted attraction to the amenities, diversity, and opportunities available elsewhere; both for themselves and their families. In addition to these practical considerations, there were significant discussions of geography and landscape, culture, a sense of purpose, and a feeling of freedom and belonging that were registered in this process and which ultimately supported young adults' decisions to stay or return to Appalachian Kentucky.

As these imaginings took place, they drew heavily on how each individual was getting place at that particular moment in their development. This included not only aspects of the place itself, but also how one was imagining themselves therein. Tyler's experience illustrates this connection well. Looking forward to life after college graduation, he was eyeing the door toward large metropolitan areas beyond the region. He revealed that this desire was based on his understanding that larger cities offer a wider array of opportunities related to both his identity as a gay male and his personal aspirations. Moreover, distance would provide him a level of freedom he didn't enjoy while being near his parents who had a strong aversion to his coming out. He shared, "I see the light at the end of the tunnel. If I can graduate, secure that job, 
and move, then I can live on my own terms...I know what I'm looking for and I can't find that in Appalachia.",

For Tyler and others, eyeing the door involved a complex blending of personal aspirations into a vision of what type of place was right for him. Several participants shared similar feelings and experiences in relation to their diverse identities and positions. Where their place of origin felt welcoming and nurturing, eyeing the door home created fewer conflicts or tensions. Yet, where home or the region at large felt restraining, locations beyond became more desirable and often, a tipping point was reached and they were propelled to leave. For some, a balance was struck between the desire for easier access to amenities and distance from their pasts by eyeing the door toward a place within the region, but back in their home communities.

As part of imagining possible futures, many presume what might happen to their family or community as a result of the various paths being contemplated. In these cases, many experienced guilt and even a sense of betrayal with the thought or act of leaving their community or region. For others, this aspect of eyeing the door became an important method through which they created meaning around the decision to stay or return. Anna's story illustrates this connection precisely. Though she reflected fondly on her childhood, she never intended to go back to her small town after high school. By that time, getting place for Anna meant recognizing there was "nothing there for me." When she thought about her community and its myriad problems, particularly with substance use, she questioned, "Do I really want to go back to that?"

As her college graduation approached, Anna set her eyes on raising a family in a more urban community with more diversity and opportunities. However, when an opportunity opened up back home, she and her husband began picturing what life 
might be like if they did return. In fact, she remembered a number of pivotal

interactions which shaped this experience:

I started to miss the little things that I took for granted. I remember one time my husband and I we were in Wal-Mart. We had come across my secondgrade teacher. She stopped me and was like, "Oh my goodness, I'm so proud of you. I've been keeping up with your progress. I know you're graduating next year." My husband was really surprised. He was like, "My teachers probably don't even know my name. This is totally different from where I'm from. It's a close-knit community. It's safe here. Everyone knows everyone. This is where I want to raise my family." Then it just hit me, "Maybe if I move back I can work and try to help." I just decided to come back to my community and try to make a positive impact, and be a positive role model for the kids here.

As a result of exchanges with both insiders and outsiders, Anna began to get place in a new way and to seriously eye the door back home. Eventually, the benefits identified outweighed any restraints. Because the position involved impacting youth through programs like substance use prevention, she developed a sense of purpose in returning.

Anna is now a mother of two who has settled into that position in her hometown. Still, she sometimes engages the process of eyeing the door, even if imagining something different only bolsters her commitments. She anticipated, "The only thing that would get me to leave would be [work in my organization] at a national level. Then I'd make the move because I feel like it would benefit the youth here too. " In this way, Anna's story also captures how eyeing the door is not a static, or one- time occurrence. Rather it is regularly engaged across the lifespan with different motivations, via an ever changing set of values and objectives.

\section{Securing Place}

The third aspect of working out place is the act of securing place. Securing place comprises the decision making, planning, and movement toward realization of one's goals. The first step therein is prioritizing the values and aspirations developed through getting place and eyeing the door. This involved establishing which pieces of 
information, which imagined possibilities, and which pros and cons would ultimately direct a person's decision. Some prioritized living and working in the region over other imagined possibilities.

The next step required setting intentions and making a decision to leave, stay, or return to the region. In doing so, some resolved or even resigned to staying. Those who were away set their intentions upon returning. Others may have felt strongly at one time that leaving was the best alternative. Sam, for example, recalled setting his intentions on returning home early on; aspirations that were informed by a positive sense of community and attraction to the lifestyle Appalachian Kentucky offered. He revealed how these feelings were only solidified by getting place while away at school:

The whole time I was in college, it was with the aim of finding something that would allow me to come back to the region. That was something I was pretty intentional about...The college years were pretty tough though. It was the first time in my life I had been outside the region for a long time... It was pretty obvious to me that I hadn't been raised up like everybody else. There were some differences in how I thought about some things versus what I saw amongst my classmates and professors. Does a fish know it's in water, right? You don't know until you're pulled out.

While Sam's foundation for returning home was strong, securing place depends on more than desires and intentions. As he shared, planning to act on these intentions required accessing resources and accepting potential losses that may occur in the process:

It ended up that a position back home came open and let me get my foot in the door...For me, that was not so much coming back to any one thing as much as it was to still be a part of the community...I know full well that I could have went and made more money or got up the career ladder a whole lot faster if I had been willing to go other place, but that wasn't the most important thing for me.

Like many participants, Sam realized that going outside the region may bring him more opportunities for advancement, but he prioritized his connections to the 
community when he made the decision to return. In these cases, individuals often find themselves waiting patiently to find their niche in the work itself. This may mean accepting lower paying positions or those with less educational requirements, even if temporarily. In other cases, this might transpire through a series of serendipitous events wherein the right position became open at the right time.

Importantly, the task of accessing resources is not limited to securing employment. This may also include securing family and friendship networks, locating housing, and even ensuring emotional readiness. For example, one participant had to determine if they were ready to return to intense family conflicts. Another participant spoke of being unable to leave home initially because they lacked the confidence and skills to be on their own. Those with partners or families had to simultaneously consider what might be attainable for them.

Some struggled considerably with securing place; lacking access to many of the previously mentioned resources. However, given the many messages about lack of opportunities in the region, many were surprised by what they were able to realize in place. A number enjoyed financial benefits by living rent or mortgage free; either with their parents or in a family member's available home. Even more found that their rural communities offered them the opportunity to find fulfilling work in their fields of practice, or in areas they were only marginally qualified for but were willing to learn. Many felt these positions were at levels of responsibility and influence beyond what might be possible for them elsewhere.

Ultimately, readiness to act on one's goals evolves over time as individuals develop the means to make a move. Some found themselves realizing their goals while others resigned to other directions. Moreover, most participants found that this access, along with their values and needs, shifted over time. Below, Tess's story is 
used to illustrate how one individual experienced this progression across all three aspects of working out place.

\section{Working out Place with Tess: "What's Five Years in a Lifetime?"}

Tess's story offers a concise illustration of working out place. Over the course of her life so far, Tess set and acted upon intentions that eventually led her to her current location within the region. Speaking of her earliest experiences filtering $\underline{\text { messages, }}, \underline{\text { developing attitudes, and challenging assumptions, }}$, she remembered feeling disappointed in the quality of her education and seemingly blind acceptance of certain values and beliefs she observed within her family and the community. Driven also by messages of staying as failure and a desire for distance, Tess eyed the door towards colleges out of state. She asserted: "I was excited to leave. I wanted to make something of myself and I didn't want to be one of those who stayed...I wanted a new identity."

Through her educational and personal pursuits, Tess spent many years confronting what lies beyond the region. While she valued this time away and all it added to her perspective, she also recalled a persistent yearning for home:

Anytime I was away I was homesick. There's something about this area that just feeds your soul. I just appreciated our culture in the way of taking care of each other... the close-knit community. One day I might say something bad about you but the next day I'll be there-- I'll bring you a casserole. Everything will be all right. I missed gathering at the table for hours or cooking in the kitchen together. I missed driving on old country roads with the windows down and no cars around. I missed the quietness.

While away, Tess drew on positive memories of place and this engaged her in a new conversation with home; or a new way of getting place. For her, sensory experiences of tasting, hearing, and feeling were particularly instructive as she compared these with her experiences of the world beyond Appalachia. 
Yet despite this warm nostalgia, it took an acute turning point to transform Tess's longing into a commitment. This occurred when she was faced with the possibility of losing a multi-generational family business without her help. Through the following passage, she described the enormity of this decision:

Honestly, I didn't really have intentions of going into my family's business...But after realizing it was in jeopardy, I had this really vivid nightmare... When I woke up, I felt by selling the business I would have huge regrets. I called my family and said, "What if I came home?" I thought, "I'll give it five years. If I'm miserable, I'll know I've tried it; I gave it my best. What's five years in a lifetime?"

In Tess's experience, eyeing the door involved not only imagining how she might save the family business, but also the grief and loss she might experience if she didn't. For her, securing place also relied heavily on accessing resources her family presented to support her return.

Though Tess's initial intentions were explicitly provisional, she since found a niche for herself back home. Over the course of this research we crossed paths multiple times and I was able to follow the growth and development of her business and other engagements through social media documentation. Through these I witnessed how her family's business contributes to the economic health and vitality of her community. I also saw that Tess expanded her economic and social investments and became committed to staying in the region. What is more, she developed a greater sense of her role therein and became regularly engaged in activities and conversations to promote a more promising economic and social future for her community.

Still, despite this evolving set of commitments, Tess admitted that getting place and eyeing the door were ongoing aspects of life in Appalachian Kentucky. She noted:

The temptation to leave is always there. It's hard to live here. The poverty is hard. The drug epidemic is hard. There aren't many food opportunities. There aren't many shopping opportunities... We just got internet for the first time two 
or three months ago and it's changed our lives. Now, I don't think about moving as much...The family business is really hard...I don't how much longer I'll be able to do that. If my other business wasn't working, I'd probably leave.

Through this acknowledgement, Tess revealed that living and working in the region is informed by ongoing engagement with the tasks of working out place. As the following section points out, additional strategies are required to maintain these commitments once made.

\section{Category 2: Keeping Peace in Place}

The second question posed in this study asked: What strategies help young adults maintain their commitments to Appalachian Kentucky? Keeping peace in place was developed to encompass three strategies young adults took in response to conflicts and obstacles faced while staying and pursuing their work. Common among such difficulties were encountering resistance to personal and professional aspirations, struggling to secure strong relationships, and feeling limited by community and regional opportunities. Individuals working for non-profit organizations and in grant funded programs were additionally burdened by a sense of uncertainty regarding how long their positions and opportunities would be available.

Three synchronous strategies were articulated in response to these experiences: dancing with difference, finding your people, and feeding the soul. Although these titles are not in-vivo quotes, they closely resemble participant phrasings and sentiments regarding the strategies they evoke to maintain their commitments. These include keeping the peace externally, within relationships, but also keeping the peace internally, through a number of personal self care strategies. Figure 4 provides a visualization of each strategy and the range of tactics that characterize them. This section uses supporting quotes and a case exemplar to describe how each was employed by young adults in their commitments to 
Appalachian Kentucky. Here, the category and strategies are italicized while individual tactics are underlined.

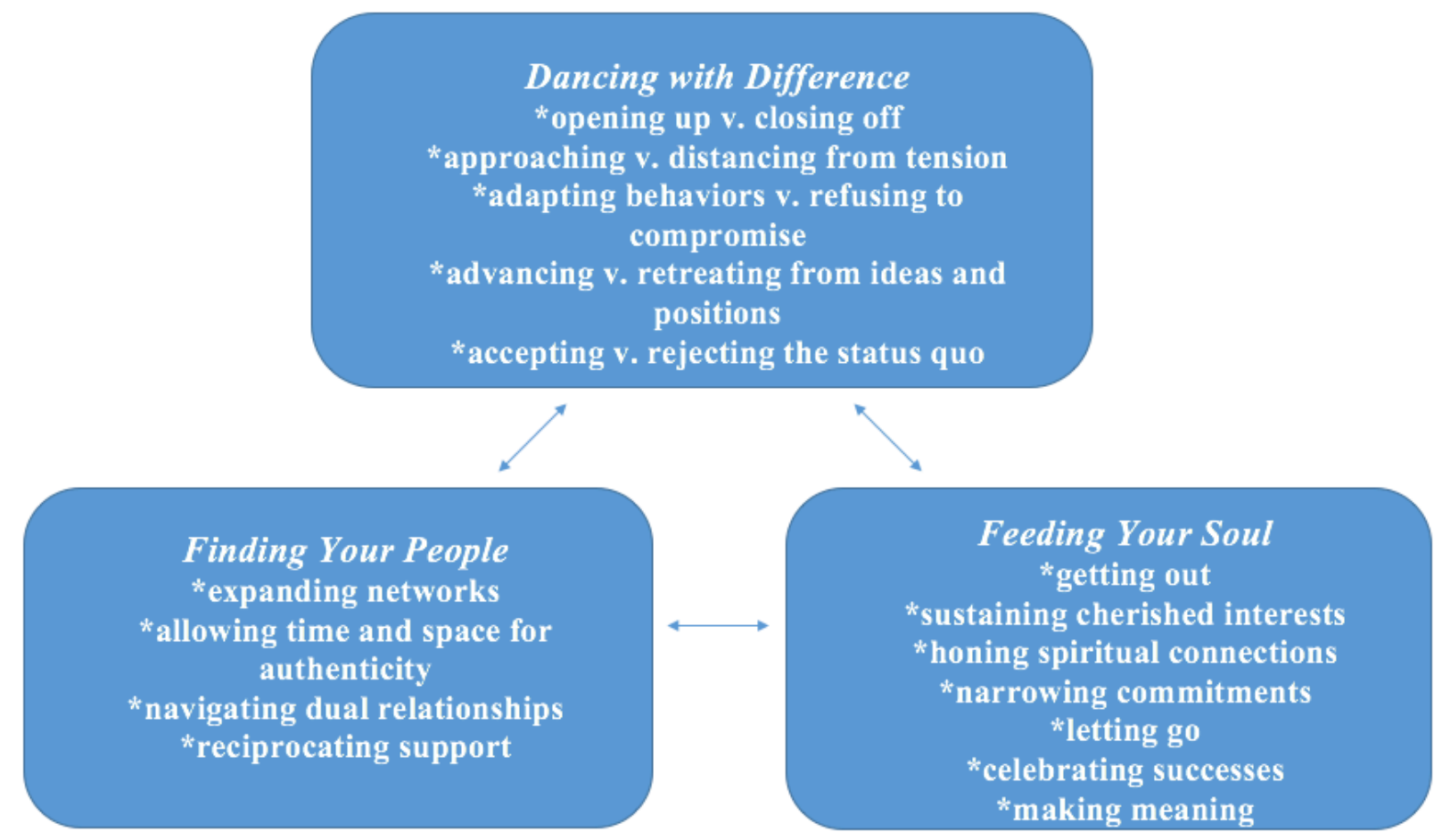

Figure 4. Keeping Peace in Place.

\section{Dancing with Difference}

Many participants found that personal positions or aspects of their identity posed challenges to their lives in the region; both private and professional. This occurred when their values, beliefs, and expressions differed from family or community norms and expectations. Dancing with difference represents how young adults move deliberately through environments and relationships which mark them as different, or wherein their differences are likely to cause conflict. While all participants engaged in these tactics to some extent, important differences emerged including: the number and type of characteristics that were managed in this way, the nature of the relationships and locations which dictated such performance, and the level of strain or conflict that either precipitated or was produced in the process. Moreover, there was variation among participants with regards to whether they were 
willing or even capable they were of making adjustments in their expression of such differences.

Often, sources of difference related to how best to behave and belong. The issues identified ranged from something seemingly small like ordering an alcoholic drink in public to identifying as an atheist in a traditionally religious family and community. Certain identities and positions caused considerable tensions in this regard. Prominent among these were gender, sexuality, religion, and politics. Several participants confronted conflicts in more than one of these areas. Jessica experienced this revealing, "There's tension between what my parents hoped I would be and what I actually became... We get along because we love each other...But it's still this process of them finding out and being disappointed anew in who I am."

One common tactic was to strategically open up and close off their identities and positions. Where differences caused considerable conflict or pain, many participants engaged in closing off these aspects of their identity. This may mean completely avoiding certain conversations, hiding aspects of identity, or trying to "pass" undetected in certain relationships. For example, Jessica shared, "There are still things my family is finding out, because I don't broadcast those things. There's just no point in ostracizing myself." She, like others, experiences this need for dancing with differences in the broader community noting:

If somebody asks me, I'm not ashamed to tell them my views. But there's no use in just creating tension for the sake of creating tension...There are some things we can talk about and some things we avoid because it's too much conflict and it's not worth it. I often deal with the tension between what I really am, what people think I am, and what most people around here are.

Like many participants, Jessica danced with differences through her gradually revealing herself and avoiding the "broadcasting" of her differences. In doing so, there is a protective element to this strategy in that it preserves relationship conflicts. 
Unlike Jessica, other young adults felt there were few, if any, differences which they kept entirely undisclosed. However, there were still characteristics which they consciously managed within their relationships. For example, several spoke of the need for distancing themselves from these conflicts and tensions, both geographically and emotionally. Aubry, for example, shared that it was important to her to be in the region, but not in her hometown based on associations community members made with her family name. In fact, this desire also compelled her to change her name upon marriage. As she explained, “There's always this moment when people from my hometown or who just generally knew our name are like, 'Oh, you're one of them.' I wanted a five-second buffer before people knew who I was. " Similar tactics emerged when young people felt they needed to establish differences between themselves and their family, from their identities as students versus professionals, or from associations with their economic status, or level of education.

Sometimes these differences were perceived as diminishing the degree of credibility one was given in their work. Other times, they facilitated entrée into certain types of work or rapport with certain populations. Knowing how to navigate these various identities across relationships and locations is part of dancing with differences. For example, Erica described adapting her behaviors and appearances while working directly with families living in poverty in the region:

You have to be humble, especially when you're dealing with folks in the rural areas...You can't go putting on airs. I'm going to show up with my blue jeans and my t-shirt, and my tennis shoes. I'm going to play with the dog on the porch and make faces at your baby while you're talking to me; relating to people on their own terms and just being humble and real.

Her experience illustrates the performative and relational nature of dancing with difference, which requires a conscious use of the self across settings. In doing so, she was able to build the support and trust needed in her work. This strategy was also 
used by individuals who needed to mask certain identities or characteristics, such as by leaving home politically affiliated clothing when attending family events.

Adapting behaviors also consisted of silencing around their differences of opinion or took an "agree to disagree" approach around many issues. However, there were also instances where this active management was either abandoned or all together rejected, particularly among individuals refusing to compromise in their expression. Russell, for example, spoke of refusing to waver in his political beliefs or to conceal his views. He shared of a times when he had posted views on social media and was confronted by neighbors, friends, and family members to take it down and avoid "stirring anything up." Sometimes, he was urged to be less outspoken and his unwillingness to do so led to changes in his relationships. He reflected, "Once they realized I was pro-this or pro-that they saw me differently or stopped coming around as much... I'm not always invited to family get-togethers...They've distanced themselves from me." Thus, Russell's experience reveals that where one does not deliberately manage identity and positions or strategically silence oneself to save face, there may be relational consequences including social isolation and a weakened support system.

Other participants discussed how advancing their ideas and positions led to burned bridges and strained relationships. Rose, for example, struggled with the differences she encountered personally and professionally with regards to her environmental beliefs and values. She reflected:

When I first came back, I guess I was kind of preachy. Then everybody shut down and wouldn't listen...It's a struggle because you have a connection to these people that you love, but you can't relate to them on some level. Or, they can't relate to you...In the beginning that was really hard for me. I mean, I would just cry...I've come to let that go...I don't talk about that much anymore. I still care a lot about the environment and try to help in my own way...I just went on with my life then they started seeing just by my example. Since then I've noticed little changes with my family... and people in the 
community or my children's school asking me questions about our lifestyle and wanting to learn. Just little things like that make me feel like there's hope.

Like Rose, many young adults adopted a new dance with difference by retreating from her ideas and positions. Rather than attempting to convert or persuade others toward her positions, she adapted her behavior toward a modeling and informative tactic; often with positive results.

Beyond personal relationships and identity characteristics, many participants were also confronted with contested visions for what was possible and desirable for the future of their organizations, communities, and the region. Where goals were common, differences also arose regarding the best process or action for achieving desired outcomes. For example, while young adults felt that everyone recognized the need for an economic strategy forward in the region, they also noted tension between new ideas and the business as usual approaches supported by many established leaders.

Similarly, there were differences of opinion among young adults regarding the effectiveness and appropriateness of activism as a mechanism for change. This is a tension experienced across the region. For example, these differences were present within several documents I reviewed pertaining to the 2018 Appalachian Studies Association's (ASA) regional conference. On the last day of the conference, heated differences arose over the appearance of author J.D. Vance among panelists at a roundtable discussion of the opioid crisis. Among the responses to this were a physical and verbal protests during the session itself, based in part on Vance's lack of professional expertise on the subject. Among those who protested were members of the Young Appalachian Leaders and Learners (Y'ALL; 2018). In a subsequent letter explaining their action, they reveal the tension between their activist approach and the expectations of others in the room noting, "[o]n multiple occasions, both convener 
and attendees who were not protesting referred to protesters as children and told them to be 'mature"" (Y'ALL, 2018). Dancing with differences thus also encompasses whether young adults accept or reject the status quo approaches to regional issues and their resolution.

Here, and in each of these tactics, it is important to emphasize that where there are two extreme ends documented (see Figure 4), many young adults would approach a situation somewhere along the continuum between these. Moreover, they may utilize different positions within a given range depending on the situation or as their understanding changes over time. For example, while some individuals rejected the status quo on principle, they indicated an interest in cultivating empathy for alternative points of view in practice. Others noted ways they circumvented the status quo by pursuing their preferred approaches discretely. Thus, interpretation of dancing with difference should include recognition of the dynamic process through which individuals selectively engage these tactics through their daily interactions in the region; all toward keeping the peace in their relationships.

\section{Finding Your People}

In addition to encountering myriad differences, young adults also noted a persistent sense of isolation that extended across multiple realms: physical and geographic, spiritual and ideological, emotional and psychological. As such, it was critical that young adults identified and sustained meaningful relationships in their communities and in their work. This need is honored in the strategy of finding your people.

The functions of finding your people include countering feelings of seclusion, sustaining social and emotional support, establishing professional encouragement, and having fun. Moreover, finding people who didn't require a constant dance with 
difference secured a sense of belonging and aided in the authenticity and longevity of relationships both personal and professional. In these ways, finding your people was an important strategy that supported young adults' ability to remain working in the region despite many challenges. As described below, a number of approaches within this process were identified.

First, although many young adults had family relationships that were supportive, most spoke of the need for expanding networks well beyond kinship ties. This included intimate relationships and friendships, as well as church communities, civic organizations, and supportive work colleagues. Having a mentor in the community or in the work was also a common experience that young adults found helpful in navigating their commitments; with the types of support provided ranging from helping someone overcome an addiction to helping a newcomer dance with difference in their work environment.

While these relationships may seem obvious or mundane, it is important to emphasize that the ease of expanding networks varies with each individual and their circumstance. In fact, several participants were initially hesitant to return to the region for fear of not building connections with like-minded others. This often occurred among those who felt their personal values, beliefs, or identities might be uncommon or even unwelcomed. Several participants also struggled with the complexity of finding lasting romantic partnerships within a small regional dating pool. Another participant's engagement was ended because his fiancé could not manage the many challenges they faced in their rural area while he remained committed to staying. Establishing professional supports presented additional challenges. For example, several young adults were working in controversial areas which caused relationship 
strain. Others worked in solitude, such as by traveling long distances to visit multisited projects.

Each type of relationship noted by participants emerged in different ways. For some, this occurred through formal organizational networks or programs. Other times, it emerged organically, through a ripple effect of personal connections, membership in churches and local organizations, and by just generally showing up for community events. Several individuals spoke of the need to allow time and space for authenticity in relationships to evolve in these ways. In Matt's hometown, for example, he and his friends tried to establish a more formal networking event to bring young professionals together and reduce isolation. for young professionals. However, they found that this approach was less effective than letting relationships evolve slowly and naturally. He admitted: We found that the best way to get new people involved is to just be like "Hey, I'm having a party. Come to my party." Aubry shared a similar sentiment when discussing the importance of finding mentors in the region to support one's work. She felt, "It's no secret. You can't artificially create relationships. They have to be real." The businesses and other community spaces created by young people acrosst he region are an important component of this, as evidenced by a piece in Time Magazine highlighting one of the region's popular restaurants as an inclusive space for authentic relationships to emerge (see House, 2018).

Due to the difficulty of finding your people in small communities, several participants spoke of their closest relationships being carried into multiple realms. For example, budding friendships were often used to foster creative thinking about what was possible in their communities and in some cases led to important professional partnerships. At the same time, navigating dual relationships posed its own challenges. Below, for instance, Ash speaks of losing important personal relationships 
as a result of professional differences, which eventually led to relocation within the region:

We couldn't have boundaries. Our communities were small. Our workplaces were small. There are only so many people who are doing these things... I lost all my relationships in that community when I left my previous organization due to conflicts...Even people I wasn't in conflict with, we had mutual friends. I just couldn't handle it. They continue to have petty relationship issues that polarize the entire community. One couple broke up and it wrecked the entire social scene. There was turf war and friendship beef because of this one breakup. It was painful because I felt like I didn't have the kinds of close relationships I needed to stay.

Like Ash, many participants cautioned that dual relationships, if not managed effectively, can jeopardize young adults' stability in place and in their work.

In the previous case, a romantic relationship jeopardized the work; but things can also go in the other direction. Several participants spoke of instances where burned bridges in professional relationships severed friendship ties. This is significant in that it narrows young adults' options for meaningful relationships and also diminishes collaboration in the work itself. Such collaboration is often critical to successful efforts in the region, where ideas and initiatives often compete for resources. What is more, reducing one's network also weakens the function of reciprocating support; which plays a critical role in maintaining relationships. This is true where each individual needs a network of like-minded and encouraging others help them succeed and they too, must give back this support. Examples shared included attending events, patronizing businesses, as well as "showing up with casseroles where they were needed."

\section{Feeding the Soul}

Given the sometimes enormous responsibility taken on by participants, the numerous challenges they faced, and the depth of meaning they assign to their commitments, feeding the soul was a critical component to what helped them to stay 
and persist in their work. Where dancing with difference and finding your people are highly relational strategies, feeding the soul represents the ways young adults are keeping peace in place outside of these relationships. This takes into account the many other ways participants nurtured their physical, emotional, psychological, and ideological needs.

While different sub-strategies were presented, often they responded to similar challenges. For example, many participants struggled in the region with limited resources like internet access, entertainment, shopping, and recreational activities. In response, one way of feeding the soul was getting out of their communities for a change of pace and scenery. Often, this also included sustaining cherished interests by accessing resources and opportunities not available locally. Rose describes a quite literal example of feeding the soul by continuing her family's personalized diet. She expressed "Food nourishes us. It's a big deal for a lot of people that move here, especially when you have had access to special foods somewhere else." Her commitment to this required traveling regularly to cities in and outside of the region as well as making special efforts to procure items locally where she could through home gardening, shopping at the farmer's market, and purchasing a Community Supported Agriculture membership. For her and her family, these activities were an important part of keeping the peace in place despite barriers. However, not all examples of feeding the soul required physical distance. Many found honing spiritual connections was important to their continuity in the region. Examples of this included finding a good fit with a local church and taking time to be with nature.

Another common set of challenges included managing the uncertainty of funding or even the sustainability of one's position or organization. Moreover, many individuals indicated that burnout in the work threatened their staying power. These 
issues were common given many young adults were being asked to engage in multiple endeavors, often beyond their position of paid employment. In response, many participants felt it was important to both narrow their commitments and to let go of certain aspects of the work that were beyond their control. What is more, nearly all participants suggested that regular celebration of successes or even a small recognition of their efforts was important to their endurance in region and in the work. Finally, critical to all of these steps is the role of making meaning, or developing a sense of purpose in the work and in one's decision to stay in Appalachian Kentucky. Aaron's story illustrates how he persists both as a community member and as a legal professional by feeding the soul using several of these approaches. To begin, he noted that where finding your people is critical, it was difficult to realize the many ideas generated among he and his friends. Accepting that change would be slow, Aaron let go of some of these long-term visions and fed his soul by narrowing his area of work and celebrating small accomplishments along the way:

You start out with these grand ideas and there's been a lot of failed groups that I've joined. But I count the little things as a success. You have to start small. For me, that means counseling clients individually, one-on-one and representing clients in court. I've seen a lot of them be successful.

In addition to how these approaches aided his work, Aaron revealed how feeding the soul was critical to combatting all the temptations to leave the region. He reflected:

Sometimes I regret coming back. I'm looking at my friends who I went to law school with and they're in much higher paying jobs or doing what I feel is much more important work...I've been paid with fried apple pies...I've been paid with "I owe you" ...I'm not jealous of my friends. I'm very happy for them. But it's like we all started on the same level in law school. I'm here dealing with someone who stole somebody's lawn mower to buy meth, verses a corporate merger... I feel like that all just adds to the story and it's all part of who I am.

While noting a number of sacrifices made through his commitments, in the end Aaron feeds his soul by making meaning out of them. He, like others, came to see how his 
work fit within the larger narrative of his life story and this nurtured the service mindset he developed to serve his community. As will be seen in the next category of results, meaning making is critical component to both keeping peace in place and working out place within the region. However, first, Daniel's story of keeping peace in place is shared to illustrate how dancing with difference, finding your people, and feeding the soul intersect in one individual's experience.

\section{"The Siren Song of the Mountains": Keeping peace in place with Daniel}

Growing up in a remote mountain community, Daniel formed a limited vision for what was possible if he stayed. He felt he couldn't pursue his desired career there, but also that his more liberal beliefs and sexuality would not be accepted. However, unexpectedly, he found his values shifting upon college graduation and he began eyeing the door back home. He remembers, "I thought about my family. I started thinking about my roots. The siren song of the mountains started pulling me back...So I answered that call." Today Daniel works in economic development and nurtures a strong commitment to Appalachian Kentucky. Nonetheless, his interviews and documents pertaining to his work and community reveal multiple threats and conflicts surrounding this goal, alongside his use of strategies for keeping peace in place.

To begin, Daniel shared multiple instances of dancing with difference as he navigated his personal and professional relationships upon his return home. Early on, he was closed off about his bisexuality, an experience that felt to him "like wearing a mask and just going through my day." However, he eventually made the choice to open up to his family and has made no secret of his same sex partnership to colleagues at work. Having received an overwhelmingly positive reception, he reflects that finding his people has been a critical component to his coming out experience and also to maintaining his work and his presence in the region, 
recognizing, "Friends and family, that is what sustains me. They are my tribe...If you don't find your people it is hard to do your work."

Whereas family, friends, and many community members have embraced Daniel's differences, anxieties still persist, particularly in his work environment. $\mathrm{He}$ shared:

I have some people in the community who disagree with my lifestyle and they have been people of influence and it has threatened my job sometimes... I don't feel like I can talk about that part of my life with my employer... I don't feel like there's any less tension or that it has been relieved. I think we found a middle ground where I can keep myself from getting in trouble, if that makes sense. That's not necessarily fair because if I was a straight man, I wouldn't have to do that. We wouldn't be having that conversation.

As a result, Daniel felt his work performance must outweigh any doubts his employer might have and limit the possibility of losing his position as a result of her disapproval. At the same time, his coming out was a positive boost to his satisfaction in his work. He noted he no longer wonders if his work colleagues are questioning his sexuality and revealed, "That was always at the forefront of my mind...I don't have to worry about that now so it's easier for me to do an even better job."

While Daniel weaves in and out of these relationships and environments, he dances with differences as needed with regards to his sexuality. However, it is important to highlight how this is just one of multiple life domains that are strategically navigated in this regard; often simultaneously. For example, Daniel spoke of how his sexual identity intersected with his religious and political beliefs and how he worked to integrate these into his lifestyle:

Throughout the entirety of my life I've yo-yoed back and forth with the church. When I got to college I was like, "I'm agnostic now." But I really didn't feel like that was who I was. I think religion was that foundation that shaped my morals and shaped my values. But I've kind of had to mold those into what corresponds to what I believe and how I feel about things. Now, I kind of have my own beliefs that are pretty traditional but non-denominational. 
Moreover, because Daniel works in economic development, he encounters a number of conflicting ideas regarding how his community should proceed, including opinions at multiple scales and across sectors. Coal for example, is a highly divisive topic in his community. Daniel shared that some leaders would like to see it up and running again, where others feel it does a disservice to the long term economic sustainability of the community and the region. Moreover, this goal conflicts with Daniel and others' desire to protect the environment and to promote recreational tourism in the region.

In response to such conflicts, Daniel highlighted that despite some changes, approaching these tensions is a carefully executed dance. He insisted "It's becoming a little bit easier to discuss with the change in influence. At the same time, we have thrived for so long on the coattails of other industries, that it became almost taboo to talk about...It's always political." While Daniel largely disagreed with the status quo, his personalized approach was to respectfully disagree and diplomatically negotiate among established leaders Still, he articulated the need for new leadership and creative visions for the future. He disclosed, "We need to continue to have leaders within our community that think outside the box, rather than going back to the way that we have always been."

To maintain his commitments, Daniel also actively feeds his soul. This includes honing spiritual connections through igniting "a new found love for Appalachia, my community, and the people here... a newfound respect for the mountains." He acts on this through hiking and connecting to the outdoors, activities he refers to as his "church" because "That's where I feel most alive." Daniel also feeds his soul by making meaning of his work, which he sees as enhancing pride in the community and changing perceptions of Appalachia. Moreover, he sees an 
explicit connection between these aims and the importance of young adults staying or returning to the region. He insisted:

If you don't stay or you don't come back, your communities begin to die. Your culture, your heritage becomes fragmented...You don't remember what's important about where you came from...If Millennials like myself don't come back, communities become stuck. There's no room for growth because there's no changing ideas. There's no way to adapt to the future. It just kind of stays stagnant.

...In the past I was disassociating with Appalachia and with my community and with Kentucky because I wanted to leave; I wanted to go away. Now, I've made peace with who I was and was able to make meaning out of the work I was doing...As the place evolves, I can evolve.

As seen through his story, Daniel's vision for the community and the region is intimately connected to his own experience as he envisions a place that is welcoming for all. He sees the links between his own transformation and that of his hometown and recognizes the complexity of both working out place and keeping peace in place. In doing so, both his work and his choice to stay are part of the transformative energy witnessed across Appalachian Kentucky.

\section{Category 3: Connecting to the Big Picture}

The final question of this study asked: How do young adults make meaning of their commitments to living and working in Appalachian Kentucky? Analysis for this question sought to identify a common process through which individuals drew inspiration and developed a sense of purpose in relation to their roles in the region. In answer to this question, connecting to the big picture was a co-constructed by the researcher and participants to display their experience with meaning making.

As mentioned previously, meaning making occurred for many as part of working out place. The meaning they associated with their work developed as they were getting place and became a driver for their decision to return or stay. Rose, for example, described how this evolved when she was eyeing the door home during 
college. Thinking of her connections to family and land, she thought "I just really want to go home and farm. [The city] is awesome. But there are so many people and there's so much going on. What real effect could I have here? " For her, meaning emerged through a sense of belonging and connectedness to her family and their farm, but also to the idea that she could make a positive impact within the region; even more so than what might be possible in a larger city.

However, as others reflected, sometimes these linkages began once an individual made the commitment to stay or return. Josh's experience reflected the latter. He noted that he didn't connect to the Appalachian context until he returned home after college, a time when many of his friends and acquaintances were also returning and hoping to make a difference in the community. He remembered everyone commiserating over what they did in their college towns and thinking up all sorts of ideas about what could be done locally. Soon, they realized they would need to take the initiative to make these things happen if they wanted to move beyond complaining. This became incorporated into Josh's meaning making of his community engagement since that time. He shared, "Don't complain about it if you're not going to do anything about it. That's been my whole thing since we moved back and have been involved in the community. But before, I never thought it was something I was going to care about." Thus for some, meaning making develops as one begins keeping peace in place.

In addition to when meaning is made, there were differences among individuals with respect to how easily such connections were made. While they may be obvious or easily made for some, Allison shared the opposite based on her experience as an administrator for multiple community projects across the region. Being somewhat removed from the direct work, she made known, "It's not always 
clear cut. I can go weeks or days at work and not really connect to the overall purpose." As a result, Allison felt she had to seek out reminders of why she does the work, such as by looking for bright spots in her organization's programming to spending time listening to staff stories. This reveals that for participants like Allison, "It's hard to see the results and the big picture when you're struggling with things day to day. " In their experience, a conscious effort was needed to formulate connections to the overall purpose with larger community and regional goals, or even to her own sense of meaning in the work.

As will be described further in this section, the meanings that develop in relation to the work and to the act of staying evolve over time and emerge differently for each individual. Yet even with these contrasts, the importance of connecting to the big picture was a pronounced feature of young adults' experiences in and with the region. This was even acknowledged by those individuals who were actively eyeing the door, such as college students who were planning to leave upon graduation. Several of these participants spoke of their commitment to return later in life. One student indicated, I really want to give back and another stated, [Someday], I just want to go back so that other people realize that they have a chance, especially younger people. Despite the ambiguity of their plans, they felt a sense of responsibility to place and attached meaning to the idea of returning and contributing to improvements down the road.

As connecting to the big picture became established, two parallel processes were identified by the researcher and corroborated by participants. The first was reflecting across time; a process whereby temporal connections are made between the past, present, and future. The second was scaling up and down; or making spatial associations across micro, mezzo, and macro level contexts and systems. Within both, 
the connections being made include those regarding the decision to stay and those pertaining to the importance of the work being done.

Figure 5 illustrates these properties and their dimensions. Properties are the characteristics within the data that differentiate them from one another (Corbin \& Strauss, 2015). In this case, the major properties are temporal and spatial. Dimensions refer to the range of positions taken within each of these properties (Corbin \& Strauss, 2015). In this case, that includes positions of the past, present, and future within the temporal property and positions of micro, meso, and macro relationships in the spatial property.

While each individual may have had a stronger orientation to one of these, often they intersected. Understanding the past and present, for example was an important part of articulating new visions for the future at multiple levels. Similarly, understanding the broader issues at scales beyond the local assisted in articulating diverse ideas on the ground. The remainder of this section offers descriptions of each property and its associated dimensions, the latter of which are underlined throughout. A case exemplar follows to illustrate how these pieces wove together for one participant.

\section{Scaling Up and Down}

Scaling up and down refers to examination of social, economic, and environmental issues within their multi-leveled contexts. In doing so, individuals generated unique stories of purpose and meaning in their work in relation to the difference they were making at various scales. Here, the intentionality of their commitments derived from sources ranging from the personal to the global; or micro to macro. 


\section{CATEGORY}

Connecting to the

Big Picture

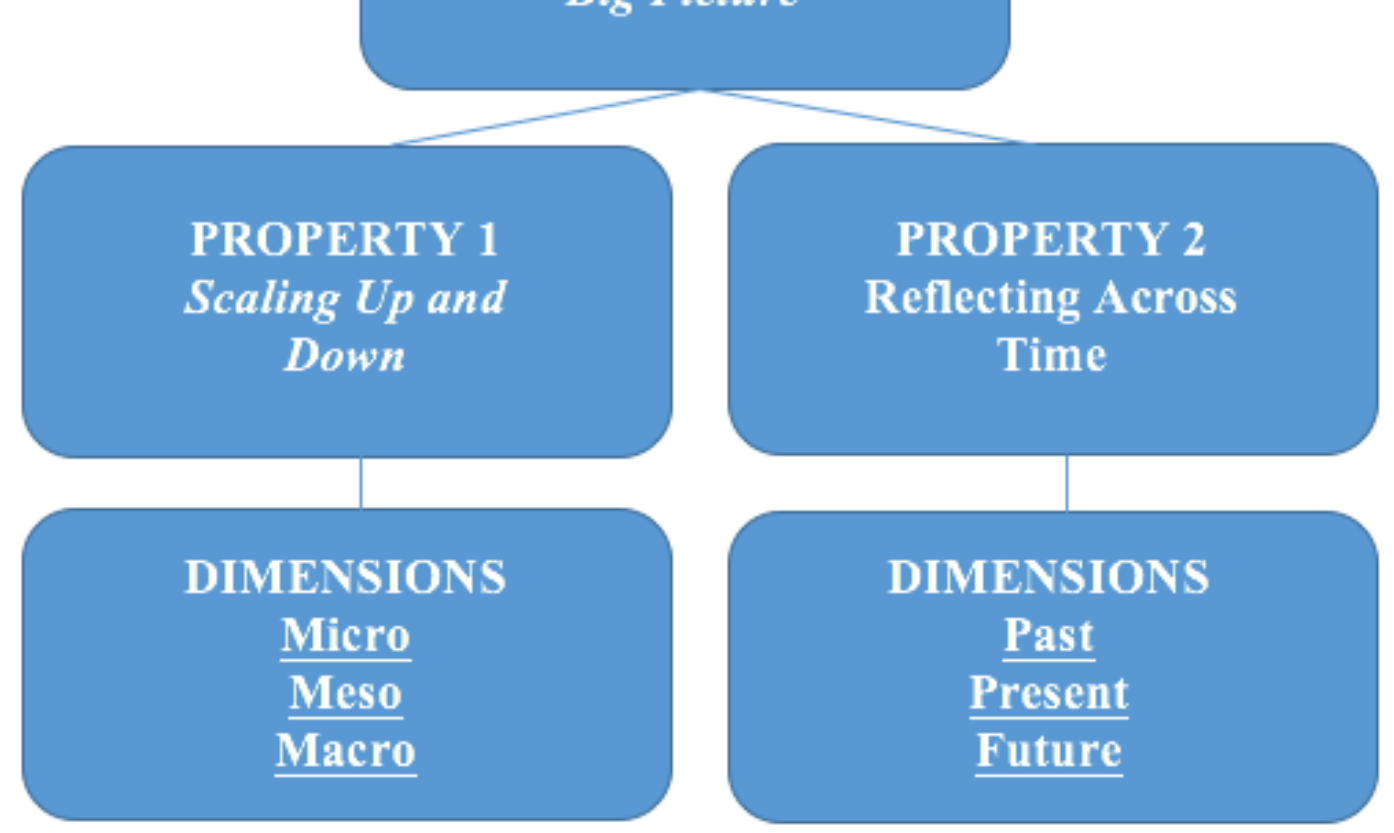

\section{Figure 5. Connecting to the Big Picture}

Scaling at the micro level involves looking for meaning and purpose at the

individual or family level. As noted before, many individuals felt it was important to stay or return to the region to care for aging parents or grandparents, as well as to create positive beginnings for their own children. Others found meaning in the specific type of work they were doing and how it shaped their identity and purpose. Phillip shared his experience with this as he reflected on what teaching in the region has meant to him personally:

I wanted to be that teacher that I didn't have...that was willing to figure out what it took for this child to succeed in the classroom...My brightest success story is a grocery store stock boy... When I got him he didn't know his last name because nobody tried... We all work to make money but it's more than a job to me. I don't see myself going anywhere from here. I think that's my life. ...Financially it gives me a paycheck, but it also satisfies what I wanted to do with my life; what makes me happy at the end of the day. When I lay down at night, I know I'm doing what I enjoy. 


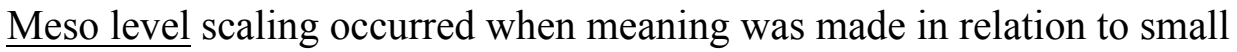
groups, organizations, and local communities. Beth for example, developed a number of meanings around her work in the arts, such as connecting to the potential social change that could be harnessed in her community workshops. In her view, "Art can be a convener across a spectrum of socio-economics, religion, race, or any of those things because we're all starting at the same level. We're sitting down with the same tools." At the same time, she encountered resistance to these meanings from individuals who don't see the value of the arts or its place within her organization. Moreover, others failed to see the need for changes that Beth felt were needed to attract more diverse community members to her organizational programming by the types of offerings, their hours, and locations of programs. As she revealed below, meaning making then extended to the institution itself, as she connected to the need for transformation at the organizational level:

Within the office, I face other people just not getting why we are putting funding toward the arts or not understanding the programs I'm doing. We've been around for over a hundred years and it's always been exactly the same: the same demographic, the same audience, the same program. It needs to change because our society isn't the same as it was a hundred years ago.

Connections to regional, state, national and international contexts are grouped into the macro level dimension. Most participants' direct impact in this dimension was through state and regional engagements, such as by advocating for policy changes or networking within one's field. However, many also had a deep understanding and affiliation with the magnitude and scope of the economic, social, and environmental issues facing the country and internationally. By grasping these issues at this scale, they were able to situate their own work and its importance within this broader landscape. The impact of coal for example, was often discussed in terms of its impact on local communities, but also in terms of clean energy futures in light 
of global predictions for climate change. Economic development was another aspect of the work which individuals readily associated with the bigger picture; even where the application was explicitly local. Greg, for example, ties the significance of his work on downtown revitalization with efforts to lift up distressed communities across the country:

It's a really good feeling. I feel nationally and even internationally connected to people. The work we're doing has relevance to the entire country...To take a small town, a coal dependent economy and do anything of value and provide jobs and hope, it's a big deal. It's not my success, it's all of our success...A lot of people have put themselves, their work, their income on the line...The Farmers market's been our biggest success by far. It's been the single thing that's injected hope in our community more than anything else. It's something that everybody can relate to. Farming in this area is important because it's the food system. People feel good about eating clean food and knowing where it came from...It spurred a whole kind of foodie movement down here. It's still in its infancy. It's just getting started.

For participants like Greg, making meaning at the macro level was an important part of generating support and feeling pride in the work. He was able to articulate how the work impacted the local community and how it fit into the bigger picture of economic transformation and resilience, nationally.

I also encountered these connections while engaging in participant observation. For example, I attended a second annual community clean-up even organized by two young men. As I spent hours picking up trash that included needles and pill bottles, I began making connections to some of the economic and social struggles in the region, but of course also to the direct environmental needs of cleaning up a littered river bank and its beaches. But when I asked one of the organizers about their intentions for what was then a second annual event, he made connections far beyond mine in scale. He explained that during the 2016 presidential election he and his friends became "frustrated and exhausted from the bickering" and that while people "talk a good game" he wanted to ask others to get out and actually 
do something for their community. In his way then, he connected this event not just to the tangible needs of the community, but to a more abstract feeling that the national political divide was something that should be addressed on the ground, locally.

At the same time, the understanding that informed these experiences and connections also sensitized many participants to the types of unintended consequences that often accompany development and change. Ash, for example, noted concern that many of the economic development activities in the region could result in the gentrification seen elsewhere; where positive growth for some meant decreased access to amenities and resources among an area's poorest residents:

I've been really paranoid about gentrification and I keep trying to talk about that here. There's this development movement going on which is awesome...I think [some communities] are doing it right. It's a community led process and it's really beautiful. But if you don't have that it can do more harm than good and it's just a few people lining their pockets. Sure, we all benefit from going to their trendy new business but what's that doing for people who are trying to pay their bills? It's not solving some of these issues that impact development.

For Ash then, there were important distinctions to make between economic development at the macro scale and a concern for economic justice and issues of deprivation and need at the micro scale. Although unique for each individual, these types of hesitations also become part of the meaning made of decisions to stay and pursue work across economic, social, and environmental domains. Moreover, their positions impacted how they aligned themselves among the many contested visions for the region's future and how it should be attained.

\section{Reflecting Across Time}

In reflecting across time, individuals developed meaning of their location in the region and the work they were doing based on their perspectives on the past, present, and future. This included making linkages across both historical and biographical time. In other words, individuals connected to their own experience, but 
also to the timelines of their community or the region at large. In this way, each instance of reflecting across time also occurred alongside scaling up and down at one or multiple levels.

To begin, many individuals felt a sense of continuity in place beyond the present contexts that informed their work. For example, many developed a sense of meaning and purpose in being the next in several generations of family farmers, teachers, civic volunteers, activists, or even members of a particular community church. In these ways, reflecting on the past assisted in the articulation of meaning in place, or a sense of knowing one's roots and feeling that one is even just "meant to $b e$ " in the region.

Still, reflecting on the past is not limited to the micro level or to one's work. Jessica, for example, tied together how both her family and community history informed the meaning she made of staying:

My family, they were some of the first settlers here. They were here before the coal mines... seven generations...There's a huge rock where settlers camped when they were coming into the county. My great, great, great, great, great uncle carved his name in the sandstone there. I can go there and touch that...There's this comfort in knowing that this place and these mountains have sustained my family that long. No matter what happens in my life I know that it can do the same for me...I feel very connected to them and places here...Now, staying here is the most important thing in my life. It's feeling like you' re part of something bigger than yourself. It's not about how my day is going today, it's about this long view of history here...I just would feel like a fish out of water anywhere else. I feel a very strong sense of belongingness. This is where I'm supposed to be.

For many, the past also aided in making meaning of regional work at broader scales. In the excerpts below, Jessica revealed what she cherished about the region's history and connected this to her vision for more inclusion and diversity in her community:

What happened here is all these mountaineers, the rugged, very spirited people came here and moved into these hollers...That's who we are, a place of people who are cutting edge, people who are on the forefront of something, 
people who are not afraid to stand alone, people who prefer that. That's who we are culturally...

That's what we want to instill in younger people coming up, that it's not about conforming. The people who came and settled these hollers weren't upstanding people back home. They came because they weren't accepted for whatever reason and they needed a place where they could spread out. That's who we've been, our cultural heritage that we need to reclaim. I feel like us staying here and being who we are, that is how a place changes.

In some ways, Jessica is unique in the level of certainty she has and in her deep and explicit efforts to articulate meaning around her commitments. However, what she shared with many other participants was a carefully balanced honor and respect for the past with acknowledgement that some elements may be best left behind moving forward. For many, in fact, there was a deeper personal meaning and a transformative potential inherent in the act of staying to articulate and represent these visions in the future. This included increasing the visibility of the rich differences and diversity present in the region across identities, positions, ideas and other characteristics. It also meant ensuring young people growing up today feel a sense that they belong and opportunities across the region.

In these ways, much of the reflection across the past and present was done in service to an imagined future at multiple scales. Beyond the importance of being present, this had a variety of direct articulations within the work ranging from installing solar panels to investing in youth. Others articulated important changes in the way progress and change were approached, such as increasing participatory, antioppressive, and justice centered approaches to economic, social, and environmental improvements. As noted, these connections across time were often multiple and occurred simultaneously with attempts to contextualize meaning at various scales. Cat's story below, beautifully illustrates these myriad components of connecting to the big picture. 


\section{"I'm going to stay here and fight": Connecting to the Big Picture with Cat}

Cat's works as an activist and advocate combines social and environmental platforms in the region. Within her organization, there is also an explicit focus on empowering young people to have an active voice in decision making, as witnessed through multiple participant observation experiences and related documents. As she shared her experiences, it became clear that her commitments to the region developed through connecting to the big picture. Cat's story is also exemplary as it demonstrates how this process informs and is informed by her experience of working out place and keeping peace in place, thus further illustrating how these categories are joined over time.

In many instances, for example, Cat reflects a strong reverence for the past and draws upon history as a source of inspiration for her work. The quote below shows how this appreciation evolved from her earliest experiences of getting place:

I grew up really hating the mountains and that cultural narrative that we're all toothless, backwards, and barefoot. In my early preteen years, I realized maybe it wasn't all bad, that there are things to love about being from here... When I got to college...that changed a lot of things for me. Being able to research and understand the histories of Appalachian resistance.... and talk about prominent activists who have come from this area has really changed my perspective. Understanding why Appalachia is represented the way it is in pop culture, recognizing Appalachia is viewed as a sacrifice zone and what that really means and how long it's been that way has put me in a position where it's like, I'm mad as hell and I'm going to stay here and fight.

For Cat, learning about the region's history of exploitation, oppression, and activism opened her eyes to a new perspective on the region. This transition was facilitated by reflecting across time and scaling up and down, in that she examined historical contexts and their impacts at micro, meso, and macro levels. Moreover, she shares how this meaning making both informed her resistance to migration and her commitment to activism. 
By the same token, Cat wrestles with the more unsettling aspects of the region's past, such as the colonization and genocide of the region's Native American population and the oppression of many minority groups over time. As she expressed:

It's difficult to talk about the past because it's traumatizing and there's a lot of reckoning with past sins that has to be done. But I also think that we're really focused on the future because we're focused on not recreating oppressive power structures. I think that the past is really important in the context of recognizing that there are elements of our culture that are good and that we can bring with us into the future...I don't want to spend too much time focusing on the negative aspects of the past. Yes, it's important to destroy the bad things and take power away from the people who are using it to abuse. But it's also important to build new things.

In these ways, the lessons of the past shape both Cat's vision for the region's future and her acute attention to the process through which these goals are obtained in the present. This includes her emphasis upon "centering the voices of the oppressed" and ensuring "everyone is around the table when we're making decisions."

Cat also demonstrated spatial connections at multiple scales. For example, having been previously set back by a medical diagnosis, the meaning made of her commitments to the region became tied to this very personal micro level circumstances. Once she was recovered, she committed to chasing down the opportunities she couldn't pursue when she was ill and a personal sense of purpose developed and helped motivate her commitments.

Still, having significant micro level connections does not preclude Cat from seeing the big picture at larger scales. For instance, she reflects that the work she is doing with young people in local communities across Appalachian Kentucky is a local example of a macro level global movement among young people to create sustainable and inclusive future for all. As she expressed, she sees her particular role within that as one that is aimed at meso level change efforts:

I understand the larger global context...I get it and this feels right and this is how I'm going to fix it at home...I think the way we understand global 
problems is through personal stories and those stories are always inherently local...There is a personal aspect of finding your place in the movement...personal stories can inform a larger system.

Not only does her own personal story connect at all these scales and across time, but like others, Cat makes connections for the region at large. Moreover, she discusses how her work aims to inspire young people to stay in the region and how the meaningfulness of this effort helps to sustain her as well:

Hope keeps me here...seeing people who are 14 or 15 come out of their shell and realize they can be a leader is what gets me out of bed in the morning...it's always transformative to see someone realize, "I am a leader" and start to consider themselves the future and act like it... We really want to empower young people to stay in KY. So that's one of my favorite parts is when you're talking to someone and they say, "Well, now I think maybe I'll stay." Or, "I was going to leave KY but now I'm thinking maybe I'll try to get a job here."

She affirms the broader significance of the work by advising to youth: The important part is that you're here and you believe that you can do it. That's an act of resistance and that's enough. In this way, she too is able to identify the transformative potential in young adults' commitments to stay.

\section{Doing Place: A Context Specific Interpretive Framework}

While the categories explored above pair neatly with the sequence of questions presented by this study, the way they actually emerge for individuals is more complex. The context specific framework of doing place was created with this in mind. It draws on the idea that the logic needed for writing up constructivist grounded theory often misaligns with the dynamics of human experience (Charmaz, 2006). This applies not only to their sequence; but also to the clarity of each category's boundaries. For example, connecting to the big picture occurs throughout the other phases. Similarly, while eyeing the door impacts whether or not individuals leave, stay, or return to Appalachian Kentucky, participants continue to experience this once they make their decision. As a result, doing place is conceptualized as 
cyclical, wherein these three processes are simultaneously shaping individuals' commitments to place (see Figure 6).

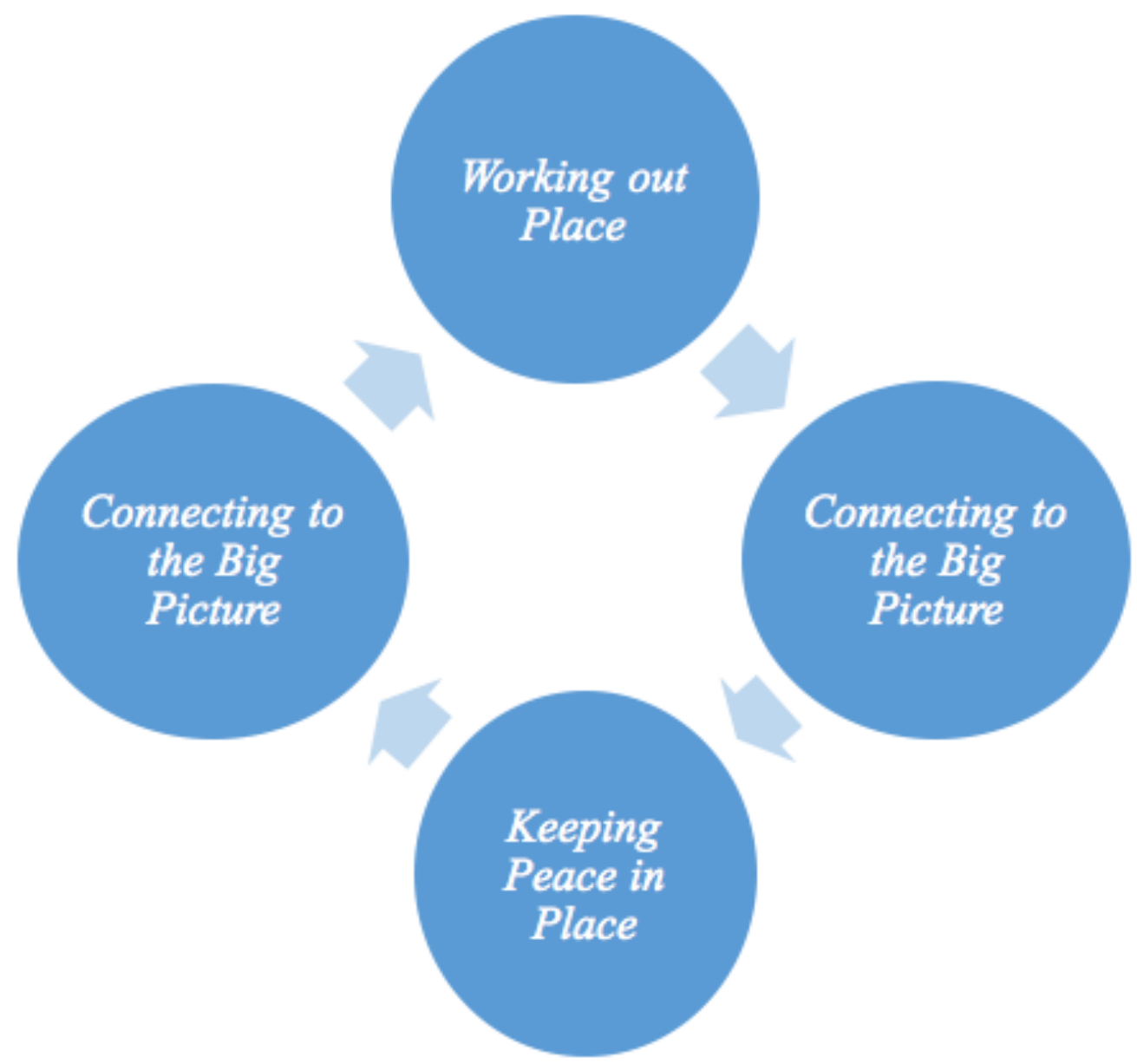

Figure 6. Doing Place

The cyclical visualization of doing place facilitates our understanding that commitments to Appalachian Kentucky are dynamic and constantly evolving. However, the succinctness of this figure should not imply that individuals are encountering the same conflicts or issues time and again. Rather, as new experiences and meanings develop, changing ideas and attitudes emerge in relation to place. These help to reinforce commitments but also to transform them as individuals begin take on the challenge of keeping peace in place. 
In sum, the components of doing place are operating simultaneously at multiple levels. Thus, the underlying concept within this context specific framework is that the commitments young adults make to Appalachian Kentucky have been deeply shaped by their experiences in place; and at the same time, these young adults are drawing on this experience to actively shape the region through their commitments. As such, this model and its components have important implications for theory, practice, education, and research. The concluding chapter provides a brief summary of these findings and discusses their implications from multiple vantage points. 


\section{CHAPTER FIVE: DISCUSSION}

This study explored the processes and meanings that inform decisions to stay in Appalachian Kentucky among college educated young adults working in areas of social, economic, and environmental importance to the region. The previous chapter fulfilled the dual aims of providing qualitative description of young adults' commitment making processes and developing a context specific framework for their interpretation. This chapter begins with four sections dedicated to each result category and the doing place framework respectively. These include a discussion of findings in relation to existing literature. The succeeding section focuses on implications for teaching, learning, and action in social work. A review of strengths, limitations, and suggestions for future research follows. A brief conclusion offers final reflections.

\section{Working out Place}

Working out place (Figure 3) underscores three prominent processes that inform young adults' decisions to stay and work in Appalachian Kentucky. The first of these is getting place. Getting place encompasses a broad range of experiences, through which individuals come to understand their communities and the region of Appalachia, as well as their own positions, identities, belonging, and sense of purpose therein. These formative experiences include both positive and negative messaging, significant events, and interactions with others within and from outside the region.

Echoing previous research, the messages received often associated movement outside of the region with individual progress and development (Dalsgaard Pedersen \& Gram, 2017; Donaldson, 1984; Gabriel, 2006; Toth, 2014). Also noted were both 
explicit and implicit messages about whether certain individuals were welcome or belonged; particularly those who presented differences in sexuality, gender, politics, and religion. As documented by scholarship from similarly distressed rural communities (e.g. Carr \& Kefalas, 2009; Easthope \& Gabriel, 2008; Wolfe et al., 2017), these formative interactions often occurred in relationship with important adults and through educational experiences. Through these, many young adults came to associate staying in the region with personal and cultural inferiority. These results thus support regional scholars who insist that exclusionary messaging within and about place has a direct impact on youth motivations to leave (Daniels, 2014; House, 2014; McMichael, 2014).

In addition to these congruencies, a surprising finding within getting place was the importance, for many participants, of their experiences outside the region. These facilitated new understandings and meaning associated with Appalachian Kentucky. This corroborates the idea that contact with 'the other,' in various forms, is a critical component of individual identity formation and meaning making (Bhatia, 2011). Rather than internalizing stereotypes or increasing physical and emotional distance from the region, such encounters often fueled desires for young adults to return and contributed to visions of positive improvements.

The second process within this category is eyeing the door. This phase is largely contemplative and includes weighing various pros and cons of a decision to stay, leave, or return. As such, this process connects directly to Value X Expectancy models of migration decision making. These propose that individuals value certain goals and weigh these based on their expectation that migration will result in their achievement (De Jong \& Fawcett, 1981). Many participants identified known “push” and "pull" factors of migration such as cost of living, career options, and educational 
opportunities. However, regional uniqueness was uncovered in such elements as the importance of generational ties, connections to landscape, and opportunities to give back to one's community. This diversity of factors emphasizes the need to balance rational choice models of migration decision making with those emphasizing structural, emotional, and imaginative processes (Castles et al., 2014; Kokkalainen \& Kyle, 2016).

The final step identified is securing place. This involves setting and acting upon intentions. According to cognitive behavioral theories (e.g., Ajzen, 1985; 2011), such intentions are the result of beliefs formed around the potential outcomes of an action This insight produces an important link between the beliefs that young people form while getting place, and their eventual decisions.

Often, however, there are discrepancies between what individuals intend to do and what actually occurs (Kley, 2017). Like any behavior, leaving, staying, or returning to Appalachian Kentucky relies on different levels of individual control, skill, and resources (Conner \& Armitage, 1998). This requires examining what Black and colleagues (2011, p. 448) term "intervening obstacles and facilitators" that impact decision making. This includes such things as cost of moving or the availability of social supports or occupational opportunities. Previous examination of the Millennial experience, for example, suggests that this cohort is "stuck" in place as a result of national economic trends (e.g., Fry, 2017). For the most part, however, participants in this study did not express a feeling of dissatisfaction or resignation with their location. In fact, many found that upon return, they encountered opportunities unavailable elsewhere; particularly in their careers and within their community. This stands in direct opposition to narratives of the region which emphasize lack of opportunity and possibility (e.g.Vance, 2016); particularly for children and youth. 
Most studies of human movement focus on the act of moving or the acculturative processes that emerge thereafter (Kokkalainen \& Kyle, 2016). This study is rare in that it included participants who were either in the early stages of decision making or were able to reflect on recent encounters with the desire to migrate but ultimately chose to stay. The phases identified fit with Kley's (2017) conceptualization of migration as a multi-staged process that should include contemplative and pre-decisional phases. As getting place illustrated, this should also consider how beliefs, attitudes, and identity formation impact this process even before active consideration occurs. What is more, some aspects of this process, such as the imaginative elements in eyeing the door, may be continuously engaged as a way of reinforcing one's decision. In this way, an additional stage of maintenance should be considered as part of this dynamic process.

\section{Keeping Peace in Place}

Consistent with existing research in Appalachia (e.g. Bell, 2013; Hatch, 2009; Wolf et al., 2017), young adults in this study experience strain with regards to the political and ideological differences in their work environments. Many also reveal that conflicts and pressures occur across their personal relationships and impact their expression of identity and purpose in place. Keeping peace in place (Figure 4) reflects three major strategies that young adults enact in order to maintain their commitments to the region despite these pressures.

Dancing with difference involves approaching relationships strategically, through decisions such as whether to be open or closed about identities, or to advance or retreat from beliefs and opinions. Dancing with difference connects to a substantial body of literature supporting the performative and relational aspects of the self (Katovich \& Reese, 1993). This includes numerous regional examples such as code- 
shifting, or changing speaking patterns in response to stigmas associated with Appalachian accents (Clark \& Hayward, 2013; Daniels, 2014; House, 2008). While many participants encountered this while outside the region, they also expressed the need for managing their self expression within the region. This finding is similar to that of Wolfe and colleagues (2017), who illustrate how young adults actively distance themselves from the stigma of staying in an economically depressed rural community. However, here, participants were more often managing other types of stigmas, such as those associated with their gender expression, sexuality, or religious and political affiliations. It may be that these participants circumvented the stigma of staying in Appalachian Kentucky as a result of their educational or occupational achievements, or in some cases, financial and social capital within their communities. Participants expressed that often, dancing with difference was used as a tactic to preserve relationships. Similar uses have been evidenced in literature related to interpersonal discussions of politics (e.g., Morey, Eveland, \& Hutchens, 2012) sexuality (e.g., Schrimshaw, Downing, Cohn, \& Siegel, 2014) and religion (e.g. McCurry, Schrotdt, \& Ledbetter, 2012). Yet for many, there was also an emotional burden and personal sacrifice embedded in the process. This is a significant consideration in terms of young adults' long term sustainability in the work and in the region; a conclusion with emerging research support. For example, Newheiser, Barreto, and Tiemersma (2017) found that non-disclosure of LGBTQ identity in the work setting actually diminished sense of belonging and self-esteem, as well as reduced job satisfaction and work commitment. And Russell (2011) shows how rural to urban migration may be a way to achieve congruity between identity and place for individuals unwilling or unable to conceal locally stigmatized identities. 
To some degree, the weight of this task is offset where finding your people and feeding your soul are successful. These strategies connect to extensive bodies of literature on the importance of self care and social support in rural practice communities (e.g., Daley \& Avant, 2014; Humble et al., 2013). However, they are especially noteworthy here for their connection to dancing with difference. Where individuals are exhausted by the performative work of relationships, these other strategies become even more important. This is critical as building these supports and outlets helps young adults establish a sense of belonging and purpose in place; which then affirms and sustains their commitments (Rosso, Dekas, \& WrzesniewskiI, 2010; Wright, 2018).

Based on these results, it appears that individuals with more intense or frequent experiences of marginalization are most in need of these connections. They also seem to be the most fervently committed to shifting local and regional narrative around inclusivity and diversity. In fact, feelings of disjointedness between identity and place may provide inspiration and motivation for their transformative ideals and practices (Erikson 1950; McLean, Wood, \& Breen, 2013; Schwartz et al., 2013; Wright, 2018).

\section{Connecting to the Big Picture}

Connecting to the big picture (Figure 5) reflects how young adults made meaning of their commitments to Appalachian Kentucky. Here, the term 'meaning' aligns with existing literature, which suggests that meaning develops when some order or sense is made an experience (Pratt \& Ashforth, 2003) and that these are largely positive associations (Rosso, et al., 2010). Critical to this development were the processes of scaling up and down and reflecting across time. 
Scaling up and down refers to the way young Appalachians see connections between personal and local challenges and those experienced at other levels, including nationally and internationally. By connecting the very personal to the global, young Appalachians understand how the challenges they face are similarly experienced the world over. Similarly, many draw upon and connect to global responses to shared impacts of globalization, deindustrialization, climate change, and political and social division in their work (Martin, 2015; Weinbaumm, 2004; Williams, 2002). By making these connections, participants articulate what place scholar Doreen Massey (1994, p. 156) described as a "global sense of the local." In other words, they conceptually deconstruct or blur the lines of geographic distinction; comprehending global trends and contexts while still being situated and active in the local (Hawkins, 2014).

Reflecting across time displays how younger Appalachians also draw upon the past, present, and future to make meaning of their experiences in the region. This process affirms that one's understanding of place, and place-based meanings lie heavily in how that place is experienced and understood in relation to time (Tuan, 1977). Meaning is constructed biographically as individuals move through and make sense of the places they occupy or encounter. Time also operates when young adults contextualize present day conditions within an historical past; expanding their meaning making across longer periods of time and beyond the scale of their own experience. Here, these imaginings were particularly focused on how the region could change in positive ways, while retaining what is treasured and meaningful about the past. These components of meaning making were important in shaping young adults' commitments to social, environmental, and economic improvements in Appalachian Kentucky. 
Consistent with the work of Rosso and colleagues (2010), participants noted a variety of sources for their meaning making in this regard including the self (e.g. values, motivations), others (e.g. coworkers, leaders), the work context (e.g. organizational mission, job tasks) and spiritual life (e.g. sacred callings). However, here, analysis emphasized what have been termed 'mechanisms of meaning' or how meaning is developed around this work (Rosso et al., 2010). According to Rosso et al., (2010), seven work meaning mechanism have been identified within the literature: authenticity, self-efficacy, self-esteem, purpose, belongingness, transcendence, and cultural and interpersonal sense-making (Rosso et al., 2010). By scaling up and down and reflecting across time simultaneously, participants here exhibited an ability to activate a mixed set of these mechanisms; constructing meaning interpersonally, relationally, and in exchange with structural and abstract components of their environments.

The ability to make meaning of this work is an important component of young adults' experience in the context of regional challenges including outmigration. Previous literature on work meaning (see Steger, Dik, \& Duffy, 2012) reveals that those who articulate a greater good or purpose in their vocation report better psychological health and well being (e.g. Arnold, Turner, Barling, Kelloway, \& McKee, 2007). Such individuals also report more satisfaction and commitment to their work (Harpaz \& Fu, 2002; Nord, Brief, Atieh, \& Doherty, 1990; Sparks \& Schenck, 2001). An additional aspect of meaning making occurred in reference to young adults' decision to stay or return to the region. For most, this meaning solidified over time and in concert with decisions regarding career, family, and other lifestyle decisions. This is consistent with their developmental stage given that 
meaning making and identity formation are particularly important during adolescence and young adulthood (Erikson, 1950; 1968; Noble-Carr \& Woodman, 2018).

The fact that many participants began to shape these meanings during major life events resonates with literature on identity construction among young people, which indicates that this is a particularly salient task during times of hardship and struggle (Kok, Goh \& Gan; 2015, McLean, Wood, \& Breen, 2013; Noble-Carr \& Woodman, 2018). Where a positive sense of identity is achieved or maintained during trying times, young people find purpose and growth (Drapeau, Saint-Jacques, Lepine, Begin, \& Bernard, 2007; Lerner, Roeser, \& Phelps, 2008). Having a positive identity is associated with personal meaning and purpose in life; both of which have long term impacts on wellbeing (Erikson, 1950; 1968; Gallagher \& Lopez, 2008; Schwartz, Zamboanga, Luyckx, Meca, \& Ritchie, 2013; Kok et al., 2015; Noble-Carr \& Woodman, 2018). In these ways, the young adults in this study illustrate the importance of meaning making to their overall commitments to place and their longterm success and sustainability in the region.

\section{Doing Place}

In concert, the results of this study provide a context specific illustration of how individuals are shaped by place and in turn, shape places (McInerney et la., 2011; Therborn, 2006). By emphasizing the action and agency embedded in this process, the 'doing' in doing place speaks to Kingsolver's (2018) insistence that in Appalachia, place is more verb than noun. This occurs through interactions within the multilayered and dynamic process of doing place (Figure 6; Saar \& Palang, 2009; Seamon, 2014). Where working out place emphasizes how place is learned, interpreted, sought, and found; keeping peace in place highlights the strategies engaged to maintain one's relationships and well-being in place. Connecting to the picture concerns how 
meaning making is infused throughout these experiences and their associated tasks and strategies. This section explores how these results confirm and extend several theoretical aspects of place.

First, doing place illustrates the importance of the three sensitizing concepts reviewed in Chapter 2 and supports their continued use in research that explore migration decisions and commitments to community engagement (e.g., Adams, 2016; Bell, 2013; Davidson et al., 2005; Marshall et al., 2012; Smith et al., 2009). Though participants did not use the language of these concepts to describe their experience, the analytic categories developed articulate that each of these inform young adults' commitments to Appalachian Kentucky; establishing important connections to theories of migration and meaning making in work. Place attachment presented itself where bonds with place evolved as young adults learned and interpreted Appalachian Kentucky over time through such processes as getting place and scaling up and down. Place identity is also represented here, as individuals were shaped by the identity of Appalachian Kentucky (Akesson et al., 2017; Altman \& Low, 1992; Cross, 2015). Territoriality sensitized this work to the inherently political nature of place and its ongoing construction (hooks, 2009; McInerney, et al., 2011) and multiple examples were shared by participants noted as they danced with differences within the region. Each of these contributed to the ability of young adults to maintain their regional commitments by contributing to a sense of belonging and purpose in place (Saar $\&$ Palang, 2009).

Second, symbolic interactionism proved its theoretical and methodological effectiveness in stressing the role relationships take in these place based experiences (Blumer, 1969). In line with existing literature, (Abrams \& Hyun, 2009; Munford \& Sanders, 2015) relationships were critical to young adults' formation of both identity 
and attachment. And it is through relationships that they are made aware of territoriality and assert themselves in relation to these conflicts (Alkon \& Traugot, 2008). Analysis in this regard was further enhanced by Cross's (2015) framework which emphasized looking for such interactions in myriad forms including narrative, historical, sensory and ideological iterations. Grounded in participants' experiences, the results developed support these theoretical underpinning and their suggestion that place is an emergent creation continuously evolving through human production and interaction (Lefebvre, 1978; Pred, 1984).

A third connection to place theory is the importance of meaning making. Though represented by connecting to the big picture, this permeated all other processes, tasks, and strategies and was critical to young adults' decisions and ability to stay and work in Appalachian Kentucky. Also, as Hawkins (2014) advises, place served as an important mediator in personal meaning making. Bendiner-Viani (2013) proposed that this meaning making occurs in three dimensions of place: where people interact with the world; where people interact with others; and where people connect the local through temporal and spatial contexts (Bendiner-Viani, 2013). Indeed, doing place provides evidence of each of these dimensions by showing how young adults made meaning through relationships, through experiences and contacts in their environments, and by crafting a sense of purpose that drew on their location across time and at various scales. Because these meanings emerge through social and cultural exchanges, this also connects place concepts to the insights of symbolic interactionism. These interactions are important to both individuals and places, leaving both open to ongoing revision and allowing their use for various social and political purposes (Austin, 2018; Kemmis, 1990; Rosso et al., 2010; Weick, 1996). 
The former insight extends the application of place concepts in important ways, including by showing the role territoriality plays in shaping identity at multiple scales. This occurs in how places are remembered, represented, and imagined in both discourse and practice. By shaping new images and crafting new narratives, the young adults here are directly impacting local culture and creating new climates in the region that ultimately transform the meanings that shape how others get place. For example, those who choose to open up about their differences provide models for youth who may question whether they belong or whether there is a space for their differences in the region. By opening businesses or to serving in social service positions, they are demonstrating that opportunities for success are possible in the region. Moreover, through their daily interactions, many are encouraging hard but necessary conversations regarding the region's economic, social, and environmental path forward among a disparate set of views for this future.

\section{Implications for Teaching, Learning, and Action}

Taken holistically, doing place fits with assertions that place is something learned (e.g., Cresswell, 2015) and enacted (e.g., Kingsolver, 2018). Building on this scaffolding, this section considers implications of this study's findings for teaching, learning, and action. Consistent with the professional and academic context from which this study was proposed, this discussion centers on applications in the field of social work. However, this should not preclude potential applications and extensions from other disciplines or perspectives.

\section{Teaching and Learning}

Formal education played a pivotal role in the lives of the young adults interviewed for this study. In many cases, the acquisition of higher education catalyzed decisions to leave the region, but it also facilitated opportunities when 
returning home. Moreover, significant learning experiences aided in a depth of understanding of regional issues as well as the development of a sense of purpose in place. For these reasons, the findings of this study affirm the broad understanding that education aids young people in conceptualizing both the self and place (Stevenson, 2008). Moreover, it suggests that intentional use of place in education could be a useful strategy for grounding learning in local experience and shaping how young people learn and engage with their communities (Smith, 2007). This has important implications for education both within and beyond social work.

Known as place-based education (PBE), pedagogies emphasizing place concentrate on student action and agency in the context of real world problems and their solutions (Rodriguez, 2008; Smith \& Sobel, 2010). Obvious among these experiences are opportunities like internships and field placements (Smith, 2002). However, it is also worthwhile to explicitly draw upon place to teach concepts in the classroom (Sobel, 2004). In either case, a guiding principle for this approach is to increase sense of place among both teachers and learners by situating knowledge acquisition within local histories, settings, contexts, and meanings (Semken, 2005), and interdisciplinary and intergenerational exchanges are prominent (Gruenewald, 2003a; 2003b; Mannion \& Adey, 2011; Stevenson, 2008). These efforts have long been important to educators emphasizing experiential and problem based forms of learning (McInerney, Smyth, \& Down, 2011).

Proponents insist that such place based connections provide opportunities for students to enhance substantive content knowledge as well as to reflect on what it means to belong to and create community (hooks, 2009; McInerney et al., 2011). This is particularly important to consider in light of this study's findings, which suggest that feelings of belonging and purpose are critical to both decisions to stay or return to 
Appalachian Kentucky, as well as commitments to engaging with pressing regional issues. Additionally, given the developmental stage of participants when they began both forming their identities and sense of place, the experiences shared here suggest that early and frequent PBE experiences may be particularly instrumental in challenging negative messages and establishing critical attachments in place. Such experiences could include formal education in public school settings, but also in other locations and domains where children and youth are intentionally engaged in action and learning within their communities.

While PBE may occur largely in the context of local experience, the learning itself is not limited in this way. Today, place based pedagogies make explicit connections between local experiences and global trends (Gruenewald \& Smith, 2014). As seen both in this study and in related literature, such linkages are critical to moving learning beyond geographic specificity in both theory and practice; particularly with regards to issues of social, economic, and environmental injustice (Anglin, 2016; Rice \& Burke, 2018). PBE scholars argue that experiences rooted in place intensify student connections with these issues, heighten their empathy and compassion, and may ignite their commitments toward positive change (Deringer, 2017; Gruenewald, 2003a; 2003b; McInerney et al., 2011). An example of such connections was seen regionally in a series of forums exploring post-coal developments in Wales with the declining coal economy in Eastern Kentucky (Eblen, 2014). Increasing access to such events and learning opportunities should be a goal of social workers and allied professionals working with rural youth in diverse settings.

Appropriately for social work and to contexts like those of Appalachian Kentucky, PBE aligns with the teaching and learning objectives of critical pedagogies which attend to issues of power (Gruenewald, 2003a; 2003b). Adopting a critical 
stance toward place adds to PBE the aims of what Gruenewald (2003a; 2003b) terms 're-inhabiting' our environments for wellness, sustainability, and justice, and 'decolonizing' place by changing how we conceive and engage within environments of exploitation and oppression (see also Huffling, Carlone, \& Benavides, 2017). This means extending student learning beyond understanding toward action and transformation; which also requires engagement beyond the classroom. Activities in this regard include facilitating opportunities for individual and collective action toward change.

Still, this must be presented with caution such that students do not perceive that either the root causes or end solutions are the sole responsibility of individuals (McInerney et al., 2011; Nespor, 2008). This has particularly strong connections to social work. As educators, we must ensure that local and personal solutions are balanced with an acceptance of the limits of individual responsibility and a strengthening of social welfare institutions and policies through legislative advocacy, and these too can be articulations of PBE in social work curriculum (Hayes-Conroy, 2008; McInerney et al., 2011)

In addition to making these connections, this study suggests that learning in and about place impacts the process of meaning making and commitment in one's work. In this regard, making meaning might be considered a critical tasks of social work, in that it holds the potential to expand human relations, compassion, and the creative exchange of ideas toward sustainable justice and equity (Hawkins, 2014, p. 90). For example, understanding diversity within and among places may facilitate deeper knowledge of disparities tied to geographic location and local culture, such as in health outcomes (Akesson et al., 2017; Bice-Wigington \& Morgan, 2018). But while place has been demonstrated here to be an important mediator in meaning 
making, it is an often neglected aspect of diversity and intersectionality, and has rarely been considered in teaching social work's seminal person-in-environment perspective (Akesson et al., 2017; Bice-Wigington \& Morgan, 2018; Kirst-Ashman \& Hull, 2015). Extending potential applications of place theory and concepts within our curriculum, is thus another important task moving forward.

A final reflection on implications for education concerns this study's context specific examination of rural change efforts among young adults. As a case study, this set of findings could fill a noted curricular gap, as such examples are needed for teaching connections between environmental, social, and economic oppressions (Belanger, 2014; Hickman, 2014) as well as how these are approached in rural practice at multiple scales (Pugh, 2007; Scherch, 2015; Slovak et al., 2011). Such explicitly rural practice content is lacking in most curriculums (Daley \& Avant, 2014; Mackie, 2012; Phillips et al., 2010). In fact, this may be one reason why social work students develop misperceptions and deep concerns regarding rural practice (Mackie and Simpson, 2007).

In this regard, the experiences revealed in this study may be offered to explore the opportunities and challenges of rural practice. Such insights may assist in the difficult task of recruiting and retaining social workers for rural practice by giving them a realistic impression of the obstacles they may face (Mackie, 2007; Mackie and Lips, 2010). For example, participants in this study encountered a number of common challenges in rural practice including competition for limited resources, working in isolation, dual relationships in the community, and an often ideologically divisive atmosphere regarding the programs and practices they wished to implement (Charania, 2011; Daley, 2015; Ginsberg, 2011; Lewis et al., 2013; Rice \& Burke, 2018). Simultaneously, they encountered personal challenges including difficulty 
making social connections and finding safe spaces to express differences. Still, the experiences of individuals in this study demonstrate that while rural practice challenges may persist, there are diverse voices and positions present among rural residents and that transformative change is possible.

\section{Action}

Findings from this study have multiple implications for social work practice. Emphasized here are the significant roles that meaning making, relationships, and resources played in young adults' experiences of doing place. These are organized by micro, meso, and macro level practice implications.

\section{Micro level.}

There are many points of entry where elements of doing place impact practice with individuals, families, and groups. One of the most significant among these is the need for belonging in close relationships. With regards to youth, this is an area of practice also supported by research in rural youth development which highlights the importance of families, educators, and mentors in youth development and decision making (Carr \& Kefalas, 2009; Easthope \& Gabriel, 2008; Wolfe et al., 2017). As participants here revealed, many experienced tension and conflict in work and family settings with regards to their beliefs, characteristics, and expressions. There is thus a role for social workers to aid young people in coping with these challenges in diverse settings where they may interact such as in schools and youth programs.

There is also a need to attend to these issues in individual and family therapy settings. This includes not only aiding young people to cope with their differences, but examining the potential for interventions that open communication and reduce tensions and conflicts around these issues; particularly those that caused the most distress such as sexuality, gender, politics, and religion. This work can build on 
existing research to develop unique applications in rural settings with youth and families. For example, Zimmerman, Smith, Simonson, \& Myers (2015) studied 'coming out' as an atheist and found that how this was experienced depended largely on the degree of flexibility or rigidity of the family member's perspective; with rigid families exhibiting less acceptance and support. These insights could be used in to develop and evaluate interventions focused on increasing flexibility among families facing similar issues.

\section{Meso level.}

The lessons learned in doing place also extend to practice in organizational and community settings. One of the most direct ways this occurs is through social workers who serve as supervisors and administrators within regional programs and organizations. They have a unique vantage point from which to apply many of the insights shared here by young adults.

To begin, they have the potential to create organizational cultures where openness about difference is intentionally welcomed. They play a part in helping young adults find their people through efforts to connect employees and coworkers through consultation, networking, collaboration, and mentorship (Ginsberg, 2011). This may also mean helping young adults to manage dual relationships and to maintain working relationships despite personal tensions and conflicts. Such social workers also have a meaningful role to play in feeding the soul of their colleagues by celebrating successes and encouraging the practice of "letting go" where possible. Given the role of meaning making, it is also interesting to consider the possibilities for organizational and community level practitioners to directly facilitate and encourage the development of work meaning in their settings. Of course, these strategies should be paired with direct provision of important resources for securing 
place such as livable wages and health insurance. All of the above mentioned strategies should be supported in an effort to increase both work satisfaction and personal wellbeing in place; which aid in retention and sustainability within organizations (Newheiser et al., 2017; Russell, 2011).

At the community level, it is also important to consider how practitioners might more actively and directly facilitate hard conversations around social, economic, and environmental conflicts. Hirsch and Dukes (2014), for example, describe how they strategically engaged diverse stakeholders to build consensus around environmental conflicts in Appalachian Virginia. Such examples could be used to build and evaluate similar approaches. These efforts could ultimately reduce conflicts and lead to improved outcomes in many of the domains that most Appalachians would like to see improve, including poverty, education, employment, and health.

Finally, the young adults in this study support the requirement that in any endeavor, the inclusion of diverse voices is critical (Drake, 2001; Eller, 2008; Fisher, 2013; Fisher \& Smith, 2012; Kingsolver, 2018). Social workers have a responsibility to seek and lift up these voices, particularly those directly impacted by marginalization and injustice. Social workers across the region can make efforts to include these groups in needs assessments, program design, and evaluation in meaningful ways that shape how they are directly impacted by programs and policies.

\section{Macro level.}

First among macro level considerations is to find unique disciplinary approaches to diminish the structural impacts of young adult outmigration, such as programs and policies that increase educational and work opportunities (Castles et al., 2014). These factors directly facilitated young adults' ability to return and engage 
meaningfully in the region. As indicated by participants here, this may mean advocating for state, regional, or national policy change such as shifting status quo economic development trends toward innovative initiatives that emphasize local diversification. Other examples include linking policy to the resources young adults need to successfully persist in the region.

There is also room to consider how social work might help to create, implement, and evaluate programs that promote positive place based messages and experiences that attend to the more emotional and psychological aspects of young adults' desire for migration (Kokkalainen \& Kyle, 2016). This might include such practices as securing funding for and implementing regional conferences and arts based programming that provide opportunities for interpersonal connection, cultural reflection, and the generation of new ideas. Or, for example, investing in environmental preservation and outdoor recreational opportunities as a link between young adults' desire to protect and connect with nature and regional economic development needs.

At the same time, there is a lesson here for the importance of encouraging and supporting programs that explicitly link young adults to places beyond their home communities and the region, to ensure the benefits of learning and experience identified here. Still yet, this should be balanced with crafting creative ways to recruit them back. Programs that explicitly link cultural exchange and travel could be particularly instructive and social work could play an important role in linking these to issues of social, economic, and environmental justice. In all of these instances, procuring funding for and evaluating such unique initiatives could be a positive role for social work at this level. 


\section{Strengths, Limitations, and Suggestions for Future Research}

Overall, this study possesses a number of strengths. Attention to the topic of young adults' commitments to Appalachian Kentucky addressed a research gap in regional scholarship, provided evidence to support theories of place, and has noted implications for social work application. By using qualitative inquiry, these insights were gained from individuals who have direct experience with these decisions and community engagements (Padgett, 2008). And as a relative "insider" within the region, I was afforded rare access to the individuals and processes under investigation. Supplementing interviews with observations and document review supported triangulation of data sources and scrupulous member checking added to the rigor and trustworthiness of these findings.

Still, two prominent limitations must be noted. The first is related to the characteristics of the participants. While inclusion criteria were purposefully narrow across several domains, the sample lacked diversity with regards to participant location within the region. As seen in Figure 2, participants were pooled in the southeastern corner of Appalachian Kentucky and many of these were located along corridors of either Interstate 75 or major regional parkways. This is partly the result of the purposive snowball sampling method, in that participants connected me to their acquaintances and colleagues for recruitment. However, it may also reflect a concentration of social, economic, and environmental change efforts in these communities and reinforce that access to major transportation routes may figure into young adults' abilities to sustain their commitments.

A second limitation is in the presentation of results. It has been noted that explication of generous context adds to rigor in qualitative research (Padgett, 2008). These broader contexts were considered in the framing of research questions, 
implementation of data collection, and during analysis. However, during the writing phase it became clear that inclusion of many contextual and situational details would compromise participant confidentiality. This limited the amount of details included about individual participants' communities, work, and personal identities. It also narrowed the scope of observations and documents included in the results, given many of these would also compromise participant confidentiality. Prioritizing this ethical commitment, emphasis was instead placed on offering rich detail of the common processes demonstrated by participants across these contexts. Three case exemplars were offered to balance this approach with the need to illustrate their application. Examples of observational and document analysis were also included in each results category.

Of course these limitations must be considered with any interpretation and use of these findings. However, they are also instructive for future research. Qualitative extensions include investigating how the processes of doing place emerge for other demographic groups within the Appalachian region. This includes those living in more remote areas, older adults, young adults without college educations, and individuals directly impacted by the work of social, economic, and environmental organizations in the region. Studies comparing the experiences of Appalachians who have migrated would also shed light on these processes and what may be different for those unable or unwilling to stay in the region. Similarly, there is a need for further understanding of what factors influence the presence and intensity of meaning making in relation to both work and place.

Where constructivist grounded theory applications can aid in these pursuits, additional qualitative approaches are also critical to our understanding. For example, future studies might successfully investigate these processes using a narrative 
approach; particularly given that identities and meanings evolve over time in a developmental and biographical way (Creswell \& Poth, 2018; McLean et al., 2013; Noble-Carr \& Woodman, 2018; Seidman, 1991). Moreover, ethnographic studies of particular organizations, communities, or specific differences or contested visions would allow for deeper understanding of the operation of these processes at scales beyond the individual and meet the need for situating these within their sociopolitical contexts. Content analyses could also be useful to understand how these conflicts are embedded in organizational and community documents and discourses.

Longitudinal studies, both qualitative and quantitative, would illustrate how these process evolve over time and could assess conditions and experiences that support and threaten commitments to stay (Piche, 2013). Additional quantitative recommendations include wider surveys of these phenomena within the region to include further operationalization and application of place attachment, place identity, and territoriality identified here and their connections and relationships with existing theoretical components of migration decision making, and community engagement (Kokkalainen \& Kyle, 2016). Sub-regional differences across these factors should also be further explored, particularly in access to resources and their connection to outmigration. Rigorous program evaluations, participatory, and community engaged research paradigms also have an important role to play in investigating these connections and lessening the gap between research and practice in each of these critical directions.

\section{Conclusion}

Over many decades, Appalachian Kentucky has faced persistent youth outmigration alongside numerous social, economic, and environmental challenges (Betz \& Partridge, 2013; Pollard \& Jacobsen, 2018). As elsewhere, this trend is driven 
by both structural and symbolic factors influencing youth desires (Black et al, 2011; Castles et al, 2014). The results of this has sometimes devastating effects, both economically and culturally and as such, many communities are interested in understanding how to mitigate or even reverse this phenomenon (Artz, 2003; Carr \& Kefalas, 2009; Heinemann \& Hadler, 2015; Wolfe et al., 2017).

This study directly attended to these issues by exploring the processes, strategies, and meanings engaged by young adults committed to social, economic, and environmental change efforts in Appalachian Kentucky. Grounded in their experience, this work established a context specific framework for interpreting these experiences titled doing place. Through this framework, we come to better understand the complexity of their journeys, the intensity of their everyday challenges, and the profundity of the meanings they associate with place. What is more, we bear witness to the palpable energy of transformation that their presence brings throughout the region. Through their many commitments to place, they pronounce that future they envision does not require abandoning the past and that staying does not equal stagnation. 


\section{REFERENCES}

Aagard, J. \& Matthiesen, N. (2016). Methods of materiality: participant observation and qualitative research in psychology. Qualitative Research in Psychology, 13, 33-46. DOI: 10.1080/14780887.2015.1090510

Abrams, L. S., \& Hyun, A. (2009). Mapping a process of negotiated identity among incarcerated male juvenile offenders. Youth \& Society, 41, 26-52.

DOI:10.1177/0

$044118 X 08327522$

Achieve. (2017). Year in review: An invigorated generation for causes and social issues. The Millennial Impact Report, 2017. Retrieved from: http://www.themillennialimpact.com

Adams, H. (2016). Why populations persist: mobility, place attachment, and climate change. Population and Environment, 37, 429-448. DOI: 10.1007/s11111015-0246-3

Adams, M. (2017). Retrieved from https://www.100daysinappalachia.com/2017/08/30/nohateinmyholler-becamewar-cry-appalachia/

Addams, J. (1922). Peace and bread in time of war. New York, NY: Macmillan.

Adler, P. A., \& Adler, P. (1994). Observational techniques. In N. K. Denzin \& Y. S. Lincoln (Eds.), Handbook of qualitative research (pp. 377-392). Thousand Oaks, CA: Sage. 
Agar, M.H. \& McDonald, J. (1995). Focus groups and ethnography. Human Organizations, 541, 78-86. DOI: 10.17730/humo.54.1.x102372362631282 Agnew, J.A. (1987). Place and politics: The Geographical Mediation of State and society. Boston, MA: Allen \& Unwin.

Aguiniga, D. M., \& Davis, A. (2014). Location matters: Using GIS mapping to address policy issues in rural areas. In T. Laine Scales, C. L. Streeter, and H. S. Cooper (Eds.), Rural Social Work: Building and Sustaining Community Capacity (2nd ed., pp. 252-266). Hoboken, NJ: Wiley \& Sons.

Ahern, M., Hendryx, M., Conley, J., Fedorko, E., Ducatman, A., \& Zullig, K. (2011). The association between mountaintop mining and birth defects among life births in central Apppalachia, 1996-2003. Environmental Research, 11, 838846. DOI: $10.1016 /$ j.envres.2011.05.019

Ahern, M., Mullett, M., McKay, K, \& Hamilton, C. (2010). Residence in coal-mining areas and low-birth-weight outcomes. Maternal and Child Health Journal, 15, 974-979. DOI: 10.1007/s10995-009-0555-1

Ajzen, I. (1985). From intentions to actions: A theory of planned behavior. In J. Kuhl \& J. Beckman (Eds.), Action-control: From cognition to behavior (pp. 1139). Heidelberg, Germany: Springer.

Ajzen, I. (2011). The theory of planned behavior: Reactions and reflections. Psychology \& Health, 26 (9), 1113-1127.

Alkon, A.H. \& Traugot, M. (2008). Place matters, but how? Rural identity, environmental decision making, and the social construction of place. City \& Community, 7(2), 97-112. DOI: 10.1111/j.1540-6040.2008.00248.x

Akesson, B., Burns, V., \& Hordyk, S-R. (2017). The place of place in social work: Rethinking the person-in-environment model in social work education and 
practice. Journal of Social Work Education 53, 372-383.

DOI:10.1080/10437797.2016.1272512

Alexander, T. (2006). Defining the diaspora: Appalachians in the great migration. Journal of Interdisciplinary History, 37, 219-247. Retrieved from http://www.jstor.org/stable/4139547

Ali, S.R. \& Saunders, J.L. (2009). The career aspirations of rural Appalachian high school students. Journal of Career Assessment, 17, 172-188. DOI: $10.1177 / 1069072708328897$

Ali, S. R., \& McWhirter, E. H. (2006). Rural Appalachian youth's vocational/educational post-secondary aspirations: Applying social cognitive career theory. Journal of Career Development, 33, 87-111.

Allen, B. (1990). The genealogical landscape and the southern sense of place. In B. Allen \& T.J. Schlereth (Eds). Sense of place: American regional cultures, (pp. 152-163). Lexington, KY: University Press of Kentucky.

Altman, I. \& Low, S.M. (1992). Place attachment. In I. Altman \& S.M. Low (Eds.), Human behavior and environment: Advances in theory and research. New York, NY: Plenum Press.

Amankwaa, L. (2016). Creating protocols for trustworthiness in qualitative research. Journal of Cultural Diversity, 23, 121-127.

Anglin, M. K. (2004). Erasures of the past: Culture, power, and heterogeneity in Appalachia. Journal of Appalachian Studies, 10(1/2), 73-84.

Anglin, M. K. (2016). Toward a new politics of outrage and transformation: Placing Appalachia within the global political economy. Journal Of Appalachian Studies, 22, 51-56. DOI:10.5406/jappastud.22.1.0051 
Anton, C.E. \& Lawrence, C. (2014). Home is where the heart is: The effect of place residence on place attachment and and community participation. Journal of Environmental Psychology, 40, 451-461. DOI:10.1016/j.jenvp.2014.10.007

Appalachian Regional Commission (2009). Subregions in Appalachia. Retrieved from https://www.arc.gov/assets/maps/related/Subregions_2009_Map.png

Appalachian Regional Commission (2015a). Appalachia then and now: Examining changes to the Appalachian region since 1965. Center for Regional Economic Competitiveness and West Virginia University. Retrieved from: https://www.arc.gov/research/researchreportdetails.asp?REPORT_ID=113

Appalachian Regional Commission (2015b). Investing in Appalachia's future: The Appalachian Regional Commission's five-year strategic plan for capitalizing on Appalachia's opportunities. Retrieved from:

https://www.arc.gov/images/newsroom/publications/sp/InvestinginAppalachia sFutureARCs2016-2020StrategicPlan.pdf

Appalachian Regional Commission (2018a). ARC-designated distressed counties, fiscal year 2018. Retrieved from https://www.arc.gov/program_areas/ARCDesignatedDistressedCountiesFiscal Year2018.asp

Appalachian Regional Commission. (2018b). Relative poverty rates in Appalachia, 2012-2016. Retrieved from https://www.arc.gov/research/MapsofAppalachia.asp?MAP_ID=143

Appleyard, D. (1979). The environment as a social symbol within a theory of environmental action and perception. American Planning Association Journal, $53,143-153$.

Appalshop. (2018). About us. Retrieved from https://www.appalshop.org/about-us/ 
Arnold, K. A., Turner, N., Barling, J., Kelloway, E. K., \& McKee, M. C. (2007). Transformational leadership and psychological well-being: The mediating role of meaningful work. Journal of Occupational Health Psychology, 12, 193203. DOI:10.1037/1076-8998.12.3.193

Artz, G. (2003). Rural area brain drain: Is it a reality? Choices: The magazine of food, farm and resource issues, $4^{\text {th }}$ Quarter. Retrieved from http://www.choicesmagazine.org/2003-4/2003-4-03.pdf

Associated Press. (December 27, 2017). In the heart of Trump country, his base's faith is unshaken. Retrieved from: https://www.pbs.org/newshour/nation/inthe-heart-of-trump-country-his-bases-faith-is-unshaken

Austin, E. K. (2018). Bringing back place: Exploring the conceptions of space, time, and place for environmental policy. Administrative Theory \& Praxis, 40(3), 211-226. DOI: 10.1080/10841806.2018.1485446

Barcus, H.R. \& Brunn, S.D. (2009). Towards a typology of mobility and place attachment in rural America. Journal of Appalachian Studies 15, 26-48. Retrieved from http://www.jstor.org/stable/41446817

Barcus, H.R. \& Brunn, S.D. (2010). Place elasticity: Exploring a new conceptualization of mobility and place attachment in rural America. Geografiska Annaler: Series B, Human Geography 92(4), 281-295. DOI: 10.1111/j.1468-0467.2010.00353.x

Batteau, A. (1982b). Mosbys and Broomsedge: The semantics of class in an Appalachian kinship system. American Ethnologist, 9, 445-466.

Batteau, A. (1982a). The contradictions of a kinship community. In R.L. Hall \& C.B. Stack, (Eds.), Holding on to the land and the Lord (pp.35-40). Athens, GA: University of Georgia Press. 
Batteau, A. W. (1990). The invention of Appalachia. Tucson, AZ: The University of Arizona Press.

Batteau, A. \& Obermiller, P. (1983). Introduction: The transformation of dependency. In A. Batteau (Ed.) Appalachia and America: Autonomy and regional dependence. Lexington, KY: University Press of Kentucky.

Bauerlein, M. (2008). The dumbest generation: How the digital age stupefies young Americans and jeopardizes our future (or, don't trust anyone under 30). New York, NY: Penguin group.

Beaver, P.D. (1986). Rural community in the Appalachian south. Lexington, KY: University Press of Kentucky.

Beckett, L. (April, 30, 2017). Neo-Nazis and anti-fascist protestors leave Kentucky after standoff. The Guardian. Retrieved from https://www.theguardian.com/us-news/2017/apr/30/neo-nazis-anti-fascistprotesters-kentucky-pikeville

Belanger, K. (2014). Capacity for conversation: Rural communities address sustainability for global impact. In T.L. Scales, C.L. Streeter, \& H.S. Coopers (Eds.). Rural social work: Building and sustaining community capacity (2nd Ed., pp. 227-236). Hoboken, NJ: Wiley \& Sons.

Bell, S.E. (2013). Our roots run deep as ironweed: Appalachian women and the fight for environmental justice. Urbana, Chicago, \& Springfield, IL: University of Illinois Press.

Bendiner-Viani, G. (2013). The big world in the small: Layered dynamics of meaning-making in the everyday. Environment \& Planning D: Society \& Space, 31(4), 708-726. https://doi-org.echo.louisville.edu/10.1068/d17810 
Berry, C. (2000). Southern migrants, northern exiles. Urbana, IL: University of Illinois Press.

Berry, W. (2013, October, 3). Wendell Berry: Poet and Prophet. Moyers \& Company. Public Broadcasting Service. Retrieved from https://www.pbs.org/video/moyers-company-wendell-berry-poet-and-prophet/

Berthelsen, C.B., Lindhardt, T., \& Frederiksen, K. (2017). A discussion of differences in preparation, performance and post reflections in participant observations within two grounded theory approaches. Scandinavian Journal of Caring Sciences, 31, 413-420. DOI: 10.1111/scs.12353

Betz, M. R. \& Partridge, M.D. (2013). Country Road Take Me Home: Migration patterns in Appalachian America and place-based policy. International Regional Science Review 36, 267-95. DOI: 10.1177/0160017612467646

Bhatia, S. (2011). Narrative inquiry as cultural psychology: Meaning-making in a contested global world. Narrative Inquiry, 21(2), 345-352.

DOI:10.1075/ni.21.2.13bha

Bice-Wigington, T. \& Morgan, K. (2018). Teaching note-Diversity and difference through a rural lens. Journal of Social Work Education, 54, 392-396, DOI: $10.1080 / 10437797.2017 .1314838$

Billings, D.B. (1999). Introduction. In D.B. Billings, N. Gurney, \& Ledford, K. (Eds), Back talk from Appalachia: Confronting stereotypes (pp. 3-10). Lexington, KY: University of Kentucky Press.

Billings, D. B. (2008). Supporting "conscious hearts" and oppositional knowledge in the struggle against mountaintop removal coal mining. Journal of Appalachian Studies, 14(1/2), 20-27.

Billings, D.B. (2016). Rethinking class beyond colonialism. Journal of Appalachian 
Studies, 22(1), 57-64. DOI:10.5406/jappastud.22.1.0057

Billings, D.B. \& Kingsolver, A.E. (Eds.) (2018). Appalachia in regional context:

Place matters. Lexington, KY: University Press of Kentucky.

Billings, D.B., Norman, G., \& Ledford, K. (Eds.) (1999). Back talk from Appalachia:

Confronting stereotypes. Lexington, KY: University of Kentucky Press.

Birt, L., Scott, S., Cavers, D., Campbell, C., \& Walter, F. (2016). Member checking:

A tool to enhance trustworthiness of merely a nod to validation? Qualitative

Health research, 26, 1802-1811. DOI: 10.1177/1049732316654870

Black, R., Adger, W.N., Arnell, N.W., Dercon, S., Geddes, A., \& Thomas, D.S.G.

(2011). The effect of environmental change on human migration. Global

Environmental Change, 21, S3-S11. DOI:10.1016/j.gloenvcha.2011.10.001

Blackley, D.J., Halldin, C.N., \& Laney, A.C. (2018). Continued increase in

prevalence of coal workers' pneumoconiosis in the United States, 1970-2017.

American Journal of Public Health, 108, 1220-1222. DOI:

10.2105/AJPH.2018.304517

Blakeney, A.B. \& Marshall, A. (2009). Water quality, health, and human occupations. The American Journal of Occupational Therapy, 63(1),46-57.

DOI:10.5014/ajot.63.1.46

Blumer, H. (1954). What is wrong with social theory? American Sociological Review, 18, 3-10. Retrieved from http://www.jstor.org/stable/2088165

Blumer, H. (1969). Symbolic interactionism: Perspective and method. Berkeley, CA: University of California Press.

Bonner, A. \& Tolhurst, G. (2002). Insider-outsider perspectives of participant observation. Nurse Researcher, 9(4), 7-20. DOI:

10.7748/nr2002.07.9.4.7.c6194 
Borak, J., Salipante-Zaidel, C., Slade, M.D., \& Fields, C.A. Mortality disparities in Appalachia: Reassessment of major risk factors. Journal of Occupational and Environmental Medicine, 54(2), 146-156.

Borman, K. \& Obermiller, P.J. (1994). From Mountain to metropolis: Appalachian migrants in American cities. Westport, CT: Bergin and Garvey.

Bowen, E., Christiadi, Deskins, J., \& Lego, B. (2018). An overview of the coal economy in Appalachia. Retrieved from https://www.arc.gov/assets/research_reports/CIE1OverviewofCoalEconomyinAppalachia.pdf

Bowen, G.A. (2006). Grounded theory and sensitizing concepts. International Journal of Qualitative Methods, 5(3), Article 2. Retrieved [date] from http://www.

ualberta.ca/ iiqm/backissues/5_3/pdf/bowen.pdf

Boyd Jr., C.C. (2004). Native Americans. In R.A. Straw \& H.T. Blethen (Eds.) High mountains rising: Appalachia in time and place (pp. 7-16). Urbana, IL: University of Illinois Press.

Brammer, J. (February 25, 2018). Could a 1940s pipeline carrying hazardous liquids in Kentucky be stopped? Feds give answer. The Lexington Herald-Leader. Retrieved from: https://www.kentucky.com/news/politicsgovernment/article202100904.html

Brasier, K.J., McLaughlin, M.K., Rhubart, D., Stedman, R.C., Filteau, M.R. \& Jacquet, J. (2013). Risk perceptions of natural gas development in the Marcellus shale. Environmental Practice, 15, 108-22. DOI: $10.1017 / \mathrm{S} 1466046613000021$ 
Breckinridge, J.P., Jones, D., Elliot, I., \& Nicol, M. (2012). Choosing a

methodological path: Reflections on the constructivist turn. Grounded Theory Review: An International Journal, 15. Retrieved from:

http://groundedtheoryreview.com/2012/06/01/choosing-a-methodologicalpath-reflections-on-the-constructivist-turn/

Brinkmann, S. (2014). Getting in touch with the world: Meaning and presence in social science. In M. Jacobsen, M. Drake, K. Keohane \& A. Petersen (Eds.), Imaginative methodologies in the social sciences: Creativity, poetics and rhetoric in social research (pp.133-154). Dorchester, UK: Ashgate Publishing.

Brown, B.B., Perkins, D. \& G. Brown. (2003). Place attachment in a revitalising neighbourhood: Individual and block levels of analysis. Journal of Environmental Psychology, 23, 259-271. DOI: 10.1016/S02724944(02)00117-2

Brown, D. L. (2002). Migration and community: Social networks in a multilevel world. Rural Sociology 67, 1-23.

Brown, D. L. \& Schafft, K. (2011). Rural People and Community in the 21st Century. Malden, MA: Polity.

Brown, G. \& Raymond, C. (2007). The relationship between place attachment and landscape

values: Towards mapping place attachment. Applied Geography, 27, 89-111.

DOI: 10.1016/j.apgeog.2006.11.002

Brown, G., Reed, P. \& Harris, C. (2002). Testing a place-based theory for environmental evaluation: An Alaskan case study. Applied Geography, 22, 49-77. DOI: $10.1016 / \mathrm{S} 0143-6228(01) 00019-4$ 
Brown, J.S. (1988). Beech Creek: A study of a Kentucky mountain neighborhood.

Berea, KY: Berea College Press.

Brown, J.S. \& Hillery Jr., G.A. (1962). The Great Migration, 1940-1960. In T.R. Ford (Ed.) The Southern Appalachian Region: A survey (pp. 54-78). Lexington, KY: University of Kentucky Press.

Brownstein, R. (2016, November 17) How the election revealed the divide between city and country. The Atlantic. Retrieved from http://www.theatlantic.com/politics/archive/2016/11/clintontrumpcity-country-divide/507902/ (last accessed 26 December 2016)

Bryant, F.C. (1981). We're all kin. Knoxville, TN: University of Tennessee Press.

Campbell, J.C. (1921). The southern highlander \& his homeland. New York, NY: Russell Sage Foundation.

Carling, J. (2002). Migration in the age of involuntary immobility: Theoretical reflections and Cape Verdean experiences. Journal of Ethnic and Migration Studies, 28, 5-42. DOI: 10.1080/13691830120103912

Carr, P. J., \& Kefalas, M. J. (2009). Hollowing out the middle: The rural brain drain and what it means for America. Boston, MA: Beacon Press.

Case, A. (2017). Reflexivity in counterspaces fieldwork. American Journal of Community Psychology, 60, 398-405. DOI 10.1002/ajcp.12196

Case, A., \& Deaton, A. (2015). Rising morbidity and mortality in midlife among white non-Hispanic Americans in the 21st century. Proceedings of the National Academy of Sciences, 112(49), 15078-15083. DOI: $10.1073 /$ pnas. 1518393112 
Castles, S., de Haas, H., \& Miller, M.J. (2014). The age of migration: International population movements in the modern world $\left(5^{\text {th }}\right.$ Ed). New York, NY: Guildford Press.

Catte, E. (2018). What you are getting wrong about Appalachia. Cleveland, OH: Belt Publishing.

Caudill, H.M. (1962). Night comes to the Cumberlands: A biography of a depressed area. Boston, MA: Little, Brown, and Company.

Chae, D.H., Clouston, S., Hatzenbuehler, M.L., Kramer, M.R., Cooper, H.L.F... (2015). Association between an internet-based measure of area racism and black mortality. PLoS One, 10(4), e0122963. DOI:

10.1371/journal.pone.0122963

Chang, J.H.Y. (2000). Symbolic interaction and transformation of class structure. Symbolic Interaction, 23, 223-251.

Charmaz, K. (2004). Premises, principles, and practices in qualitative research: Revisiting the foundation. Qualitative Health Research, 14, 976-993. DOI:10.1177/1049732304266795

Charmaz, K. (2006). Constructing grounded theory. Thousand Oaks, CA: Sage.

Charmaz, K. (2008). Constructionism and the grounded theory. In, J.A. Holstein \& J.F. Gubrium (Eds). Handbook of Constructionist Research (pp. 397-412). New York, NY: The Guilford Press.

Charmaz, K. (2014). Constructing grounded theory ( $2^{\text {nd }}$ Ed.). Los Angeles, CA: Sage. Charmaz, K. (2017). The power of constructivist grounded theory for critical inquiry. Qualitative Inquiry, 23, 34-45. DOI: 10.1177/1077800416657105

Charania, G. R. (2011). Grounding the global: A call for more situated practices of pedagogical and political engagement. $A C M E, 10(3): 351-371$. 
Chen, S-H. (2011). Power relations between the researcher and the researched: An analysis of native and nonnative ethnographic interviews. Field Methods, 23(2), 119-135. DOI: $10.1177 / 1525822 X 10387575$

Chenoweth, E., \& Galliher, R. V. (2004). Factors influencing college aspirations of rural West Virginia high school students. Journal of Research in Rural Education, 19(2). Retrieved from http://www.jrre.psu.edu/articles/19-2.pdf

Choi, J.H., Zhu, J., \& Goodman, L. (July 11, 2018). The state of Millennial home ownership. Urban Wire: Housing and Finance. Urban Institute. Retrieved from: https://www.urban.org/urban-wire/state-millennial-homeownership

Christian Appalachian Project. (November 17, 2017). Tackling poverty in Appalachia. America's Charities. Retrieved from: https://www.charities.org/news/tackling-poverty-appalachia

Clarke, A. E. (2005). Situational analysis: Grounded theory after the postmodern turn. Thousand Oaks, CA: Sage.

Clark, C., Burnham, A.J., Harto, C.B. \& Horner, R.M. (2012). The technology and policy of hydraulic fracturing and potential environmental impacts of shale gas development. Environmental Practice, 14(4), 249-61. DOI: $10.1017 / \mathrm{S} 1466046612000415$

Clifford, J. (1986a). Introduction: Partial truths. In J. Clifford \& G. E. Marcus (Eds). Writing Culture: The poetics and politics of ethnography (pp. 1-26). Berkeley, CA: University of California Press.

Clifford, J. (1986b). On ethnographic allegory. In J. Clifford \& G. E. Marcus (Eds). Writing Culture: The poetics and politics of ethnography (pp. 98-121). Berkeley, CA: University of California Press. 
Collins, T. \& Dewees, S. (1999). “It don’t come EZ”: A case study of participatory research in the Jackson County, KY Empowerment Zone. Sociolotical Practice: A Journal of Clinical and Applied Research, 1(2), 157-171. Retrieved from http://www.jstor.org/stable/43735753

Conner, M. \& Armitage, C.J. (1998). Extending the theory of planned behavior: a review and avenues for further research. Journal of Applied Social Psychology, 28(15), 1429-1464.

Cooley, C.H. (1902). Human nature and the social order. New York, NY: Charles Scribner's Sons.

Corbett, M. \& Forsey, M. (2017). Rural youth out-migrationand education: Challenges to aspirations discourse in mobile modernity. Discourse: Studies in the cultural politics of education, 38, 429-444. DOI:

$10.1080 / 01596306.2017 .1308456$

Corbin, C. (2016, April 8). With collapse of coal industry, central Appalachian towns struggle to survive. Fox News. Retrieved from: http://www.foxnews.com/us/2016/04/08/with-collapse-coal-industry-centralappalachian-towns-struggle-to-survive.html

Corbin, J. \& Strauss, A. (2015). Basics of qualitative research: Techniques and procedures for developing grounded theory (4th Ed.). Thousand Oaks, CA: Sage.

Coyne, C.A., Demian-Pompescu, C., \& Friend, D. (2006). Social and cultural factors influencing health in Southern West Virginia: A qualitative study. Preventing Chronic Disease: Public Health Research, Practice and Policy, 3(4), 1-8.

Cresswell, T. (2015). Place: An introduction ( $2^{\text {nd }}$ Ed.). Chinchester, West Sussex: John Wiley \& Sons, Ltd. 
Creswell, J. W. \& Poth, C.N. (2018). Qualitative inquiry and research design:

Choosing among five approaches. Thousand Oaks, CA: Sage.

Crooks, D. L. (2001). The importance of symbolic interaction in grounded theory research on women's health. Health Care for Women International, 22, 11-27. DOI:10.1080/073993301300003054

Cross, J.C. (2015). Processes of place attachment: An interactional framework. Symbolic Interaction, 28, 493-520. DOI: 10.1002/SYMB.198

Crotty, M. (2013). The foundations of social research: Meaning and perspective in the research process. Thousand Oaks, CA: Sage.

Cuba, L. \& Hummon, D.M. (1993). A place to call home: Identification with dwelling, community and region. The Sociological Quarterly, 34, 111-131. Retrieved from http://www.jstor.org/stable/4121561

Cunningham, R. (2010). Reflections on identity and the roots of prejudice. Appalachian identity: A roundtable discussion. Appalachian Journal, 38(1), 74-76. Retrieved from: https://www.jstor.org/stable/40934852?seq=1\#page_scan_tab_contents

Daley, M. R. (2015). Rural social work in the 21st century. Chicago, IL: Lyceum Books.

Daley, M.R. \& Avant, F.L. (2014). Down-home social work: A strenths-based model for rual practice. In T.L. Scales, C.L. Streeter, \& H.S. Coopers (Eds.). Rural social work: Building and sustaining community capacity $\left(2^{\text {nd }}\right.$ Ed., pp. 5-18). Hoboken, NJ: Wiley \& Sons.

Daley, M. R. \& Pierce, B. (2011). Educating for rural competence: Curriculum concepts, models, and course content. In L. H. Ginsberg (Ed.), Social work in rural communities (5th ed., pp. 125-140). Alexandria, VA: CSWE. 
Dalsgaard Pedersen, H. \& Gram, M. (2018). The brainy ones are leaving: The subtlety of (un)cool places through the eyes of rural youth. Journal of Youth Studies, 2, 620-635, DOI: 10.1080/13676261.2017.1406071

Daniels, S. (2014). Stay here anyway. Journal Of Appalachian Studies, 20(2), 136138. DOI: 10.5406/jappastud.20.2.0136

Davidson, J., Bondi, L., \& Smith, M. (Eds.) (2005). Emotional Geographies. New York, NY: Routledge.

Davis, D.E. (2000). Where there are mountins: An environmental histoyr of the southern Appalachians. Athens, GA: University of Georgia Press.

Davis, J.E. (2004). "Lord, we're just trying to save your water": Environmental activism and dissent in the Appalachian South. Journal of American History, $91,340-342$.

DeJong, G.F. \& Fawcett, J.T. (1981). Motivations for migration: An assessment and a value-expectancy research model. In G.F. De Jong \& R.W. Gardner (Eds.). Migration decision making: Multidiscplinary approaches to microlevel studies in developed and developing countries, 13-58. New York, NY: Pergamon Policy Studies on International Development.

DePoy, E. \& French Gilson, S. (2007). The human experience: Description, explanation, and judgment. Lanham, MD: Rowman \& Littlefield Publishing Group, Inc.

Deringer, A.S. (2017). Mindful place-based education: Mapping the literature. Journal of Experiential Education, 40, 333-348.

Devine-Wright, P. (2011). Place attachment and public acceptance of renewable energy: A tidal energy case study. Journal of Environmental Psychology 31, $336-43$. 
Dimock (2018). Defining generations: Where Millennials end and post-Millennials begin. Pew Research Center. Retrieved from: http://www.pewresearch.org/fact-tank/2018/03/01/defining-generationswhere-millennials-end-and-post-millennials-begin/

Dingwall, R., DeGloma, T., \& Newmahr, S. (2012). Editors' introduction: Symbolic interaction - Serving the whole interactionist family. Symbolic Interaction, 35(1), 1-5. DOI:10.1002/symb.6

Dominis, J., photographer. (1964, January 31). The valley of poverty. Life Magazine. Retrieved from https://books.google.com/books?id=_FMEAAAAMBAJ\&pg=PA63\&source= gbs_toc_r\&cad $=2 \# \mathrm{v}=$ onepage $\& \mathrm{q} \& \mathrm{f}=$ false

Donaldson, G. A. Jr (1984). Growing Up Means Moving Away: Developmental Dilemma for the Rural Adolescent. Distributed by ERIC Clearinghouse, [Washington, D.C.]

Dorgan, H. (1987). Giving glory to God in Appalachia: Worship practices of six Baptist sub-denominations. Knoxville, TN: University of Tennessee Press. Dorgan, H. (1989). The Old Regular Baptists of Central Appalachia: Brothers and sisters in hope. Knoxville, TN: University of Tennessee Press.

Drake, R.B. (2001). A history of Appalachia. Lexington, KY: University of Kentucky Press.

Drapeau, S., Saint-Jacques, M., Lepine, R., Begin, G., \& Bernard, M. (2007). Processes that contribute to resilience among youth in foster care. Journal of Adolescence, 30, 977-999. DOI:10.1016/j.adolescence.2007.01.005 
Drew, E.M. \& Schoenberg, N.E. (2011). Deconstructing fatalism: Ethnographic perspectives on women's decision making about cancer prevention and treatment. Medical Anthropology Quarterly, 25(2), 164-182.

Dwyer, C. \& Limb, M. (2001). Introduction: Doing qualitative research in geography. In. M. Limb \& C. Dwyer Eds. Qualitative methodologies for geographers: Issues and debates. Oxford University Press, inc. New York, New York.

Easthope, H. \& Gabriel, M. (2008). Turbulent lives: Exploring the cultural meaning of regional youth migration. Geographical research, 46(2), 172-182. DOI: $10.1111 / \mathrm{j} .1745-5871.2008 .00508 . \mathrm{x}$

Eblen, T. (September 28, 2014). Forums look at what KEntucy can learn from former Welsh coalfields. The Lexington Herald-Leader. Retrieved from: https://www.kentucky.com/news/business/article44512542.html

Edwards, B., \& Addae, R. (2015). Ethical decision-making models in resolving ethical dilemmas in rural practice: Implications for social work practice and education. Journal of Social Work Values \& Ethics, 12, 88-92. Retrieved from http://jswve.org/download/2015-1/articles/88-JSWVE-12-1Rural\%20Practice-Resolving\%20Ethical\%20Dilemmas.pdf

Eller, R.D. (1982). Miners, millhands, and mountaineers: Industrialization of the Appalachian South, 1880-1930. Knoxville, TN: The University of Tennessee Press.

Eller, R.D. (2001). Forward. In D.B. Billings, N. Gurney, \& Ledford, K. (Eds.), Back talk from Appalachia: Confronting stereotypes (pp. 1-2). Lexington, KY: University of Kentucky Press. 
Eller, R.D. (2004). Modernization, 1940-2000. In R.A. Straw \& H.T. Blethen (Eds.) High mountains rising: Appalachia in time and place (pp. 197-220). Urbana, IL: University of Illinois Press.

Eller, R.D. (2008). Uneven Ground: Appalachia since 1945. Lexington, KY: University Press of Kentucky.

Ellis, C. \&Flaherty, M.G. (1992). An agenda for the interpretation of lived experience. In E. Ellis \& M.G. Flaherty (Eds.), Investigating subjectivity: Research on lived experience (pp. 1-16). Newbury Park, CA; Sage.

Engelhardt, E. D. (2017). Trying to get Appalachia less wrong. Southern Cultures, 23(1), 4-9.

DOI: $10.1353 /$ scu.2017.0001

Ergood, B. \& Kuhre, B.E. (1991). (Eds.) Appalachia: Social context past and present. $\left(3^{\text {rd }}\right.$ Ed). Dubuque, IO: Kendall-Hunt Publishing Company.

Erikson, E. H. (1950). Childhood and society. New York, NY: W. W. Norton.

Erikson, E. H. (1968). Identity: Youth and crisis. New York, NY: Norton.

Estep, B. (August 4, 2017). What would it take to fix Eastern Kentucky's economy? 30,000 jobs. The Lexington Herald-Leader. Retrieved from https://www.kentucky.com/news/state/article165563837.html

Estep, B. (July 20, 2018). Rate of choking black-lung disease hits 25-year high in Kentucky, Central, Appalachia. The Lexington Herald-Leader. Retrieved from: https://www.kentucky.com/news/state/article215225350.html

Evans, R., Ribbens, M.J., Bowlby, S., Wouango, J., \& Kébé, F. (2017). Producing emotionally sensed knowledge? Reflexivity and emotions in researching responses to death. International Journal of Social Research Methodology, 20, 585-598. DOI: 10.1080/13645579.2016.1257679 
Fairness Campaign. (2018). About us. Retrieved from:

https://www.fairness.org/about-us/

Farris, M.F. (2015). The persistence of place in Appalachia: The phenomena of postdeath migration, 1930-1970. Online Theses and Dissertations. 257. Retrieved from: https://encompass.eku.edu/etd/257

Farrugia, D., Smyth, J., \& Harrison, T. (2016). Affective topologies of rural youth embodiment. Sociologia Ruralis, 56(1), 116-132. DOI:10.1111/soru.12077

Feather, C. (1998). Mountain People in a Flat Land: A Popular History of Appalachian migration to Northeast Ohio, 1945-1965. Athens, OH: Ohio University Press.

Feldman, D.L. \& Moseley, L. (2003). Faith-based environmental initiatives in Appalachia: Connecting faith, environmental concern and reform. Worldviews: Global Religions, Culture \& Ecology, 7, 227-252. DOI: $10.1163 / 156853503322709128$.

Fielding, T. (1992): Migration and culture. In T. Champion, T. \& T. Fielding (Eds) Migration Processes and Patterns, Vol. 1: Research Progress and Prospects (pp. 201-212). London: Belhaven.

Finlay, L. (2002). Negotiating the swamp: The opportunity and challenge of reflexivity in research practice. Qualitative Research, 2, 209-230. DOI: $10.1177 / 146879410200200205$

Fischer, P. A., Holm, E., Malmberg, G. \& Straubhaar. T. (2000). Why do people stay? Insider advantages and immobility. HWWA Discussion Paper 112. Hamburgisches Welt-Wirtschafts-Archiv (HWWA) Hamburg Institute of International Economics.

Fisher, S.L. (1993). Fighting back in Appalachia: Traditions of resistance and 
change. Philadelphia, PA: Temple University Press.

Fisher, S.L. \& Smith, B.L. (Eds.) (2012). Transforming Places: Lessons from Appalachia. Urbana, Chicago, \& Springfield, IL: University of Illinois Press.

Fisher, S.L. \& Smith, B.L. (2016). Internal colony- are you sure? Defining, theorizing, organizing Appalachia. Journal of Appalachian Studies, 22(1), 4550. DOI:10.5406/jappastud.22.1.0045

Fleming A.R., Ysasi N.A., Harley D.A., Bishop M.L. (2018) Resilience and strengths of rural communities. In Harley D., Ysasi N., Bishop M., \& Fleming A. (Eds.) Disability and Vocational Rehabilitation in Rural Settings (pp. 117-136). Springer, Cham. DOI: 10.1007/978-3-319-64786-9_7

Florida, R. L. (2002). The rise of the creative class: And how it's transforming work, leisure, community and everyday life. New York, NY: Basic books.

Foster, S.W. (1988). The past is another country: Representation, historical consciousness, and resistance in the Blue Ridge. Berkeley, CA: University of California Press.

Fox, J. (1999). Mountaintop removal in West Virginia: An environmental sacrifice zone. Organizations and Environment, 12, 163-183. DOI: $10.1177 / 1086026699122002$

Fragoso, A.D. \& Townes, C. (2016, June 16). Kentucky lawmakers want to revitalize coal country by building a prison. Think Progress. Retrieved from: https://thinkprogress.org/kentucky-lawmakers-want-to-revitalize-coal-countryby-building-a-prison-73e2b5c1e166/

Fanklin, B.A. (September 27, 1981). Saving Appalachia: Was $\$ 15$ billion well spent? 
The New York Times. Retrieved from:

https://www.nytimes.com/1981/09/27/us/saving-appalachia-was-15-billionwell-spent.html

Fraley, J. (2011). Missionaries to the wilderness: A history of land, identity, and moral geography in Appalachia. Journal of Appalachian Studies, 17(1/2), 2841.

Frost, T. (January 10, 2018). Update: State of emergency declared in Martin County. WYMT Mountain News. Retrieved from:

https://www.wymt.com/content/news/State-of-emergency-declared-in-MartinCounty-468712563.html

Fry, R. (2017). Americans are moving at historically low rates, in part because Millennials are staying put. Pew Research Center. Retrieved from: http://www.pewresearch.org/fact-tank/2017/02/13/americans-are-moving-athistorically-low-rates-in-part-because-millennials-are-staying-put/

Gabriel, M. (2006). Youth migration and social advancement: How young people manage emerging differences between themselves and their hometown. Journal of Youth Studies, 9(1), 33-46.

Gallagher, M. W., \& Lopez, S. J. (2008). Positive psychology. In S. Davis \& W. Buskist (Eds.), 21st century psychology: A reference handbook, (pp. II-202-II210). Thousand Oaks, CA: Sage.

Gaventa, J. (1980). Power \& powerlessness: Quiescence and rebellion in an Appalachian valley. University of Illinois Press, Urbana \& Chicago.

Gaventa, J. (2018). The power of place and the place of power. In D.B. Billings \& A. E. Kingsolver (Eds.) Appalachia in regional context: Place matters (pp. 91110). Lexington, KY: University Press of Kentucky. 
Geertz, C. (1973). The Interpretation of Cultures. New York, NY: Basic Books.

Gergen, K. J. (2009). An invitation to social construction ( $2^{\text {nd }}$ Ed). London, UK: Sage Publications.

Gerrish, K. (1997). Being a 'marginal native': Dilemmas of the participant observer. Nurse Researcher, 5(1), 25-34. DOI: 10.7748/nr.5.1.25.s4

Gildersleeve, R. E. (2010). Dangerously important moment(s) in reflexive research practices with immigrant youth. International Journal of Qualitative Studies in Education, 23, 407-421. DOI: 10.1080/09518398.2010.492809

Ginsberg, L.H. (2005). Social work in rural communities (4th Ed.) Council on Social Work Education. Alexandria, VA.

Ginsberg, L.H. (Ed.) (2011). Social work in rural communities (5th ed.). Alexandria, VA: CSWE.

Giuliani, M.V. (2003). Theory of attachment and place attachment. In B. MiriHa, T. Lee, \& M. Bonaiuto (Eds). Psychological theories for environmental issues (pp. 137-170). New York, NY: Ashgate.

Glaser, B.G. (1978). Theoretical sensitivity: Advances in the methodology of grounded theory. Mill Valley, CA: The Sociology Press.

Glaser, B. G. (2005). The grounded theory perspective III: Theoretical coding. Mill Valley, CA: The Sociology Press.

Glaser, B. G. \& Strauss, A. L. (1967). The discovery of grounded theory: Strategies for qualitative research. Chicago, IL: Aldine Publishing Company.

Goffman, E. (1963). Stigma: Notes on the management of spoiled identity. Englewood Cliffs, NJ: Prentice-Hall.

GoTranscript. (2018). The importance of confidentiality in transcripts. Retrieved from: https://gotranscript.com/confidential-transcription-services 
Gregory, J.N. (2005). The southern diaspora: How the great migrations of black and white southerners transformed America. Chapel Hill, NC: The University of North Carolina Press.

Griffey, M. (2018). Retrieved from:

https://www.highergroundinharlan.com/blog/may-17th-2018

Griggs, K.O. (2002). The removal of blacks from Corbin in 1919: Memory, perspective, and the legacy of racism. The Register of the Kentucky Historical Society, vol. 100, no. 3, 2002, pp. 293-310.Retrieved from https://www.jstor.org/stable/23384408?seq=1\#page_scan_tab_contents

Gruenewald, D. A. (2003a). Best of both worlds: A critical pedagogy of place. Educational Researcher, 32, 3-12. DOI:10.2307/3700002

Gruenewald, D. A. (2003b). Foundations of place: A multidisciplinary framework for place-conscious education. American Educational Research Journal, 40, 619654. DOI:10.3102/00028312040003619

Gruenewald, D. A., \& Smith, G. A. (Eds.). (2014). Place-based education in the global age: Local diversity. New York, NY: Psychology Press.

Guba E. \& Lincoln Y. (1989). Fourth Generation Evaluation. Newbury Park, CA: Sage Publications.

Guba, E., \& Lincoln, Y. (1994). Competing paradigms in qualitative research. In N. Denzin \& Y. Lincoln (Eds.), Handbook of qualitative research (pp. 105-117). London, UK: Sage.

Guillemin, M., \& Gillam, L. (2004). Ethics, reflexivity, and ethically important moments in research. Qualitative Inquiry, 10, 261-280. DOI: $10.1177 / 1077800403262360$ 
Gusfield, J. R. (2003). A journey with symbolic interaction. Symbolic Interaction, 26, 119-139. DOI: $10.1525 /$ si.2003.26.1.119

Gustafson, P. (2001a). Meanings of place: Everyday experience and theoretical conceptualizations. Journal of Environmental Psychology, 21, 5-16. DOI: 10.1006/jevp.2000.0185

Gustafson, P. (2001b). Roots and routes exploring the relationship between place attachment and mobility. Environment and Behavior, 33, 667-86. DOI: $10.1177 / 00139160121973188$

Gustafson, P. (2014). Place attachment in an age of mobility. In L.C. Manzo \& P. Devine-Wright (Eds), Place attachment: Advances in theory, methods and applications (pp 37-48). New York, NY: Routledge.

Haddox, J. C. (2018). Impact of design on patient participation in healthcare in a rural Health clinic in Appalachia: A qualitative pilot study. Health Environments Research \& Design Journal, 11(1), 31-39. https://doiorg.echo.louisville.edu/10.1177/1937586717696701

Halperin, R.H. (1990). The livelihood of kin: Making ends meet "The Kentucky Way." Austin, TX: University of Texas Press.

Hammar, T. \& Tamas, K. (1997). Why do people go or stay? In T. Hammar, G. Brochmann, K. Tamas, K. \& T, Faist (Eds.) International migration, immobility and development: Multidisciplinary perspectives (pp.1-20). Oxford, UK: Berg.

Hammer, B., Fletcher, F., Hibbert, A. (2017). Participant observation: Enhancing the impact measurement in community based participatory research. Qualitative Report, 22(2), 439-455. Retrieved from https://nsuworks.nova.edu/tqr/vol22/iss2/6 
Hammersley, M. (1990). Reading ethnographic research: A critical guide. London, UK: Longmans.

Hammersley, M. (1992). What's wrong with ethnography? Methodological explorations. London, UK: Routledge.

Hammersley, M. \& Atkinson, P. (2007). Ethnography: principles in practice (3rd Ed.). New York, NY: Routledge.

Haraway, D. (1988). Situated knowledges: The science question in feminism and the privilege of partial perspective. Feminist Studies, 14, 575-99. DOI: $10.2307 / 3178066$

Harkins, A. \& McCarroll, M. (2019). Appalachian reckoning: A region responds to Hillbilly Elegy. Morgantown, WV: West Virginia University Press.

Harney (1873). A strange land and peculiar people. Lippincott's Magazine of Popular Literature and Science, 12(31), 429-437. Retrieved from: https://www.gutenberg.org/files/13964/13964-h/13964-h.htm\#strange

Harpaz, I., \& Fu, X. (2002). The structure of the meaning of work: A relative stability amidst change. Human Relations, 55, 639-667.

DOI: $10.1177 / 0018726702556002$

Harrington, M. (1962). The other America: Poverty in the United States. New York, NY: Macmillan.

Harvey, D. (1996). Justice, nature and the geography of difference. London, UK: Blackwell.

Hatch, E. (2009). A new company in town: Lessons on local identity and community development in the mountain south. In S. Keefe (Ed.) Participatory development in Appalachia: Cultural identity, community, and sustainability (pp. 45-65). Knoxville, TN: University of Tennessee Press. 
Hauge, A.L. (2007). Identity and place: A critical comparison of three identity theories. Architectural Science Review, 50, 44-

51, DOI: $10.3763 /$ asre.2007.5007

Hausmann, C. (2011). Interaction ritual theory and structural symbolic interactionism. Symbolic Interaction, 34, 319-329. DOI:10.1525/si.2011.34.3.319

Hawkins, M. R. (2014). Ontologies of place, creative meaning making and critical cosmopolitan education. Curriculum Inquiry, 44(1), 90-112. https://doiorg.echo.louisville.edu/10.1111/curi.12036

Hayes, R. \& Oppenheim, R. (1997). Constructivism: Reality is what you make it. In T. Sexton \& B. Griffin (Eds.) Constructivist thinking in counseling practice, research and training (pp. 19-41). New York, NY: Teachers College Press.

Hayes-Conroy, J. (2008). Book review: Place-based education in the global age:

Local diversity. Journal of Research in Rural Education, 23(2), 1-4.

Haynes, A.F. (1997). Poverty in Central Appalachia: Underdevelopment and exploitation. New York, NY: Garland Publishing, Inc.

Hedges, C. \& Sacco, J. (2012). Days of destruction, days of revolt. New York, NY: Nation Books.

Heinemann, L. \& Hadler, M. (2015). Resisting economic opportunities? An inquiry into the reasons and motivations of individuals who stay in an economically deprived area. Journal of Appalachian Studies, 21, 86-104. DOI: 10.5406/jappastud.21.1.0086

Hendryx, M. (2009). Mortality from heart, respiratory, and kidney disease in coal mining areas of Appalachia. International Archives of Occupational and Environmental Health, 82, 243-249. DOI: 10.1007/s00420-008-0328-y

Hendryx, M. (2011). Poverty and mortality disparities in Central Appalachia: 
Mountaintop mining and environmental justice. Journal of Health Disparities Research and Practice, 4(3), 44-53. Retrieved from:

https://digitalscholarship.unlv.edu/jhdrp/vol4/iss3/6

Hendryx, M. \& Ahern, M.M. (2008). Relations between health indicators and residential proximity to coal mining in West Virginia. American Journal of Public Health, 98, 669-671. DOI: 10.2105/AJPH.2007.113472

Hendryx, M., Ahern, M.M., \& Nurkiewicz, T.R. (2007). Hospitalization patterns associated with Appalachian coal mining. Journal of Toxicology and Environmental health, Part A: Current Issues, 70, 2064-2070. DOI: $10.1080 / 15287390701601236$

Hendryx, M. \& Innes-Wimsatt, K.A. (2013). Increased risk of depression for people living in coal mining areas of Central Appalachia. Ecopsychology, 5(3), 179187.

Hendryx, M., O’Donnell, K., \& Horn, K. (2008). Lung cancer mortality is elevated in coal-mining areas of Appalachia. Lung Cancer, 62, 1-7. DOI: 10.1016/j.lungcan.2008.02.004

Hendryx, M. \& Zullig, K. J. (2009). Higher coronary heart disease and heart attack morbidity in Appalachian coal mining regions. Preventative Medicine, 49, 355-359. DOI: 10.1016/j.ypmed.2009.09.011

Hickman, S.A. (2014). Rural is real: History of the National Rural Social Work Caucus and the NASW professional policy statement on rural social work. In T.L. Scales, C.L. Streeter, \& H.S. Coopers (Eds., pp. 19-28). Rural social work: Building and sustaining community capacity ( $2^{\text {nd }}$ Ed.). Hoboken, NJ: Wiley \& Sons.

Hicks, G.L. (1976). Appalachian Valley. Prospect Heights, IL: Waveland Press. 
Hill Collins, P. (2015). Intersectionality’s definitional dilemmas. Annual Review of Sociology, 41, 1-20. DOI: 10.1146/annurev-soc-073014-112142

Hinkley, S. (May 18, 2017). The place in America where (almost) no one drinks their tap water. The Christian Science Monitor. Retrieved from: https://www.csmonitor.com/USA/Politics/2017/0518/The-place-in-Americawhere-almost-no-one-drinks-their-tap-water

Hirsch, S.F. \& Dukes, E.F. (2014). Mountaintop mining in Appalachia: Understanding stakeholders and change in environmental conflicts. Athens, OH: Ohio University Press.

Hodkinson, P. (2005). "Insider research"' in the study of youth cultures. Journal of Youth Studies, 8, 131-49. DOI: 10.1080/13676260500149238

Højholt, C. \& Kousholt, D. (2014). Participant observation of children's communities -exploring subjective aspects of social practice. Qualitative Research in Psychology, 11, 316- 34. DOI:10.1080/14780887.2014.908989

Hook, D. (2001). Discourse, knowledge, materiality, history: Foucault and discourse analysis. Theory \& Psychology, 11, 521-47. DOI:10.1177/0959354301114006 hooks, b. (2009). Belonging: A culture of place. New York: Routledge. hooks, b. (2014). Call and response - Taking a stand. Journal of Appalachian Studies, 20(2), 122-123.

Hoover, S., \& Morrow, S. L. (2015). Qualitative researcher reflexivity: A follow-up study with female sexual assault survivors. The Qualitative Report, 20, 14761489. Retrieved from: http://nsuworks.nova.edu/tqr/vol20/iss9/10

House, S. (2014). Our secret places in the waiting world: Or, a conscious heart, continued. Journal of Appalachian Studies, 20, 103-121.

House, S. (July 26, 2018). A place for us all. Time magazine. Retrieved from: 
Huffling, L.D., Carlone, H.B., \& Benavides, A. (2017). Re-inhabiting place in contemporary rural communities: Moving toward a critical pedagogy of place. Cultural Studies of Science Education, 12, 33-43.

Humble, M. N., Lewis, M. L., Scott, D. L., \& Herzog, J. R. (2013). Challenges in rural social work practice: When support groups contain your neighbors, church members, and the PTA. Social Work with Groups, 36, 249-258. DOI:10.1080/01609513.2012.753807

Humphrey, R. (1984). Religion and place in Southern Appalachia. In P.D. Beaver \& B.L. Purrington (Eds.) Cultural adaptations in mountain environments (pp. 122-141) Athens, GA: University of Georgia Press.

Imperiale, S. (2017, April 19). Opposition to prison is an environmental fight in Appalachia. The Natural Resource Defense Council. Retrieved from: https://www.nrdc.org/experts/sara-imperiale/opposition-prison-environmentalfight-appalachia

Inscoe, J.C. (2004). Slavery and African Americans in the Nineteenth Century. In R.A. Straw \& H.T. Blethen (Eds.) High mountains rising: Appalachia in time and place (pp. 30-45). Urbana, IL: University of Illinois Press.

Jacquet, J.B., Guthrie, E., \& Jackson, H. (2017). Swept out: Measuring rurality and migration intentions on the Upper Great Plains. Rural Sociology, 82, 601-627. DOI: $10.1111 /$ ruso. 12145

Jacobson, D. (2002). Place and belonging in America. Baltimore, MD: Johns Hopkins University Press.

Jacoby, R. (2014). Cosmopolitanism and place. In W.M. McClay, \& T.V.McAllister. (Eds.), Why place matters: Geography, identity, and civic life in modern America (pp.70-83). New York, NY: Encounter Books. 
Johnson, F. (2014). Claiming a place on earth: The conscious heart of Silas House. Journal of Appalachian Studies, 20, 151-153.

Jones, L. (1994). Appalachian values. Ashland, KY: The Jesse Stuart Foundation. Jones, O. (2005). An ecology of emotion, memory, self and landscape. In J. Davidson, L. Bondi, \& M. Smith (Eds.) Emotional Geographies (pp. 205-218). New York, NY: Routledge.

Jorgensen, B.S. \& Stedman, R.C. (2001). Sense of place as an attitude: Lakeshore owners' attitudes toward their properties. Environmental Psychology 21, 23348. DOI: $10.1006 /$ jevp.2001.0226

Jorgensen, B.S. \& Stedman, R.C. (2006). A comparative analysis of predictors of sense of place dimensions: Attachment to, dependence on, and identification with Lakeshore Properties. Journal of Environmental Management, 79, 31627. DOI: 10.1016/j.jenvman.2005.08.003.

Juris, J. (2007). Practicing militant ethnography with the movement for global resistance (MRG) in Barcelona. In S. Shukaitis and D.Graeber (Eds.) Constituent imaginations (pp. 164-78). Oakland, CA: AK Press.

Kaplan, T. (March 4, 2016). This is Trump country. The New York Times. Retrieved from: https://www.nytimes.com/interactive/2016/03/04/us/politics/donaldtrump-voters.html

Kaplan, B.H. (1971). Blue ridge: An Appalachian community in transition. Morgantown, WV: Appalachian Center, West Virginia University. Kaplan, T. (March 4, 2016). This is Trump Country. The New York Times. Retrieved from: https://www.nytimes.com/interactive/2016/03/04/us/politics/donaldtrump-voters.html 
Katovich, M. \& Reese, William A., II. (1993). Postmodern thought in symbolic interaction: Reconstructing social inquiry in light of late-modern concerns. The Sociological Quarterly, 34, 391-411. Retrieved from http://www.jstor.org/stable/4121104

Keefe, S.E. (1998). Appalachian Americans: The formation of "reluctant ethnics." In, G.R. Campbell (Ed), Many Americas: Critical perspectives on race, racism, and ethnicity (pp. 129-153). Dubuque, IA: Kendall-Hunt Publishers.

Keefe, S.E. (2005). Appalachian cultural competency: A guide for medical, mental health, and and social service professionals. Knoxville, TN: University of Tennessee Press.

Kemmis, D. (1990). Community and the politics of place. Norman, OK: University of Oklahoma Press.

Kentuckians for the Commonwealth. (2018). What do we mean by a just transition for Appalachia? Retrieved from http://kftc.org/issues/what-do-we-mean-justtransition-appalachia

Kentucky Coal Association. (March 14, 2012). Governor announces that more than 50,000 'Friends of Coal' license plates have been purchased in three years. Business Wire. Retrieved from https://www.businesswire.com/news/home/20120314006568/en/GovernorBeshear-Announces-50000-Friends-Coal-License

Kim, W-Y. (2013). Induced seismicity associated with fluid injection into a deep well in Youngstown, Ohio. Journal of Geophysical Research: Solid Earth, 118 (7): 3506-18. DOI: 10.1002/jgrb.50247

Kingsolver, A. (2018). "Placing" futures and making sense of globalization on the edge of Appalachia. In D.B. Billings \& A.E. Kingsolver (Eds). Appalachia in 
regional context: Place matters (17-48). Lexington, KY: University Press of Kentucky.

Kirst-Ashman, K., \& Hull, H. (2015). Understanding generalist practice. Toronto, Canada: Cengage.

Kley, S. (2017). Facilitators and constraints at each stage of the migration decision process. Population Studies, 71, 35-49. DOI:

$10.1080 / 00324728.2017 .1359328$

Knauff, W. S. (2006). Herbert Blumer's theory of collective definition and the battle over same-sex marriage: An analysis of the struggle to control the meaning of marriage in America from a symbolic interaction perspective. Journal of Human Behavior in The Social Environment, 14(3), 19-43.

DOI:10.1300/J137v14n0302

Koch, T. (2006). Establishing rigour in qualitative research: The decision trail. Journal of Advanced Nursing, 53, 91-103. DOI: 10.1111/j.13652648.2006.03681.x

Kok, J. K., Goh, L. Y., \& Gan, C. C. (2015). Meaningful life and happiness: Perspective from Malaysian youth. The Social Science Journal, 52, 69-77. DOI:10.1016/j.soscij.2014.10.002

Kokkalainen, S. \& Kyle, D. (2016). Imagining mobility: The prospective cognition question in migration research. Journal of Ethnic and Migration Studies, 42, 759-776. DOI: 10.1080/1369183X.2015.1111133

Kornbluh, M. (2015). Combatting challenges to establishing trustworthiness in qualitative research. Qualitative Research in Psychology, 12, 397-414. DOI: 10.1080/14780887.2015.1021941 
Kotarba, J. A. (2014). Symbolic interaction and applied social research: A focus on translational science. Symbolic Interaction, 37, 412-425.

DOI:10.1002/symb.111

Kozlowski, M., \& Perkins, H. A. (2016). Environmental justice in Appalachia Ohio? An expanded consideration of privilege and the role it plays in defending the contaminated status quo in a white, working-class community. Local Environment, 21(10), 1288-1304. DOI: 10.1080/13549839.2015.1111316

Krase, J. (1979). Stigmatized places, stigmatized people: Crown heights and prospectLefferts gardens. In R. S. Miller (Ed.), Brooklyn USA: The fourth largest city in America (pp. 251-262). New York, NY: Brooklyn College Press.

Kratzer, N.W. (2015). Coal mining and population loss in Appalachia. Journal of Appalachian Studies, 21, 173-188. DOI: 10.5406/jappastud.21.2.0173

Kusow, A.M. (2003). Beyond indigenous authenticity: Reflections on the insider/outsider debate in immigration research. Symbolic Interaction, 26, 5919. DOI: $10.1525 /$ si.2003.26.4.591

Laitinen, H., Kaunonen, M., \& Åstedt-Kurki, P. (2014). Methodological tools for the collection and analysis of participant observation data using grounded theory. Nurse Researcher, 22, 10-15. DOI: 10.7748/nr.22.2.10.e1284.

Lal, B. B. (1995). Symbolic interaction theories. American Behavioral Scientist, 38, 421.

Lane, N.M., Holmes, G.M., Arcury, T.A., Schwalbe, M., Randolph, R., Frank, J..(2018). Creating a culture of health in Appalachia. Disparities and bright spots. Appalachian Regional Commission. Retrieved from: https://www.healthyky.org/res/images/resources/BrightSpotsCaseStudiesJuly2018.pdf 
Larabee, R.V. (2002). The risk of going observationalist: Negotiating the hidden dilemmas of being an insider participant observer. Qualitative Research, 2(1), 97-122. DOI: 10.1177/1468794102002001641

Larson, O.F. (1943). Wartime migration and the manpower reserve on farms in Eastern Kentucky. Rural Sociology, 8(2), 148-161.

Lauder, M. A. (2003). Covert participant observation of a deviant community: Justifying the use of deception. Journal of Contemporary Religion 18, 185-96. DOI: $10.1080 / 1353790032000067518$

Lefebvre, H. (1978). Space and the State. In N Brenner \& S Elden (Eds.). G Moore, N Brenner and S Elden (Translators), Henri Lefebvre, State, Space, World: Selected Essays. Minneapolis: University of Minnesota Press.

Lerner, R., Roeser, R., \& Phelps, E. (2008). Positive development, spirituality and generosity in youth: An introduction to the issues. In R. Lerner, R. Roeser, \& E. Phelps (Eds.), Positive youth development and spirituality: From theory to practice (pp. 3-24). West Conshohocken, PA: Templeton Foundation Press.

Letcher Governance Project. (2016). Press release: Letcher Governance Project holds silent protest at SOAR Innovation Summit in opposition to proposed federal prison. Retrieved from: https://www.thestayproject.com/blog-stay-in-thenews/press-release-from-our-allies-at-letcher-governance-project

Lewicka, M. (2011). Place attachment: How far have we come in the last 40 years? Journal of Environmental Psychology, 31, 207-230. DOI:

10.1016/j.jenvp.2010.10.001 
Lewis, M. L., Scott, D. L., \& Calfee, C. (2013). Rural social service disparities and creative social work solutions for rural families across the life span. Journal of Family Social Work, 16, 101-115. DOI:10.1080/10522158.2012.747118

Lewis, O. (1959). Five Families: Mexican Case Studies in the Culture of Poverty. New York: Basic Books.

Lewis, R.L. (1999). Beyond isolation and homogeneity: Diversity and the history of Appalachia. In D.B. Billings, G. Norman, \& K. Ledford, K. (Eds.), Back talk from Appalachia: Confronting stereotypes (pp. 21-44). Lexington, KY: University of Kentucky Press.

Lewis, R.L. (2004). Industrialization. In R.A. Straw \& H.T. Blethen (Eds.) High mountains rising: Appalachia in time and place (pp. 59-73). Urbana, IL: University of Illinois Press.

Lewis, H. M., Johnson, L. \& Askins, D. (Eds.). (1978). Colonialism in modern America:The Appalachian case. Boone, NC: Appalachian Consortium Press.

Li, J. (2008). Ethical challenges in participant observation: A reflection on ethnographic fieldwork. Qualitative Report, 13, 100-115. Retrieved from https://nsuworks.nova.edu/tqr/vol13/iss1/8

Lichterman, P. (1998). What do movements mean? The value of participantobservation. Qualitative Sociology, 21, 401-418. DOI: 10.1023/A:1023380326563

Lichterman, P. (2002). Seeing structure happen: Theory-driven participant observation. In B. Klandermans \& S. Staggenborg (Eds.) Methods of social movement research (pp. 118-45). Minneapolis, MN: University of Minnesota Press

Lincoln, Y.S. \& Guba, E.G. (1985). Naturalistic inquiry. Beverly Hills, CA: Sage. 
Link, B. G., Wells, J., Phelan, J. C., \& Yang, L. (2015). Understanding the importance of "symbolic interaction stigma": How expectations about the reactions of others adds to the burden of mental illness stigma. Psychiatric Rehabilitation Journal, 38(2), 117-124. DOI:10.1037/prj0000142

Lipson, J. G. (1984). Combining researcher, clinical and personal roles: Enrichment or confusion? Human Organization, 43, 348-352. Retrieved from http://www.jstor.org/stable/44126386

Liu, F. (2013). Migration patterns among young adults in the United States:

Environmental, social and economic explanations. Master's Thesis, Michigan Technological University, Retrieved from https://digitalcommons.mtu.edu/cgi/viewcontent.cgi?article $=1676 \&$ context $=$ et ds

Lofland, J. \& Lofland, L. (!995). Analyzing social settings: a guide to qualitative observation and analysis $\left(3^{\text {rd }}\right.$ Ed.). Belmont, CA: Wadsworth.

Loftus, T. (February 13, 2019). Bill 'effectively killing solar industry in Kentucky’ zips through Senate. The Courier-Journal. Retrieved from: https://www.courier-journal.com/story/news/politics/2019/02/13/kentuckylegislature-solar-bill-moves-seante-floor/2859997002/

Low, S. M. (1992). Symbolic ties that bind: Place attachment in the plaza. In I. Altman \& S. Low (Eds.). Place attachment (pp. 165-86). New York: Plenum Press.

Low, S. \& Altman, I. (1992). Introduction. In I. Altman \& S. Low (Eds.). Place attachment (pp. 1-12). New York: Plenum Press. 
Lowrey, A. (2014, June 26). What's the matter with Eastern Kentucky? It's the economy. The New York Times Magazine. Retrieved from http://www.nytimes.com/2014/06/29/magazine/whats-the-matter-with-easternkentucky.html?module $=$ Search\&mabReward $=$ relbias\%3Ar\&_r $=1$

Luebke, B.H., \& Hart, F. H. (1958). Migration from a southern Appalachian community. Land Economics, 34(1), 44-53. DOI: 10.2307/3144444

Luttrell, W. (2000). 'Good enough' methods for ethnographic research. Harvard Educational Review, 70, 499-523. DOI: 10.17763/haer.70.4.5333230502744141

MacFarquhar, L. (October 10, 2016). In the heart of Trump country. The New Yorker. Retrieved from: https:/www.newyorker.com/magazine/2016/10/10/in-theheart-of-trump-country

Mackie, P.F.E. (2007). Understanding educational and demographic differences between rural and urban social workers. Journal of Baccalaureate Social Work 12(3), 114-128.

Mackie, P.F.E. (2012). Social work in a very rural place: A study of practitioners in the upper peninsula of Michigan. Contemporary Rural Social Work, 4, 63-90. Retrieved from https://cornerstone.lib.mnsu.edu/cgi/viewcontent.cgi?referer=https://www.goo gle.com $/ \&$ httpsredir $=1 \&$ article $=1005 \&$ context $=$ sowk_fac_pubs

Mackie, P. F. E. \& Lips, R. A. (2010). Is there really a problem with hiring rural social service staff? An exploratory study among social service supervisors in rural Minnesota. Families in Society: The Journal of Contemporary Social Services, 91, 433 - 439. DOI: 10.1606/1044-3894.4035 
Mackie, P. F. E., \& Simpson, C. (2007). Factors influencing social work students' perceptions about rural-based practice: A pilot study. Journal of Rural Mental Health, 31(2), 5-22.

Magilla Entertainment. (Production Company). (2011-). Moonshiners [Television series]. Silver Springs, MD: The Discovery Channel.

Maitlis, S., \& Lawrence, T. (2007). Triggers and enablers of sense giving in organizations. Academy of Management Journal, 50, 57-84.

Mander, J. \& Goldsmith, E. (Eds.) (1996). The case against the global economy and for a turn toward the local. San Francisco, CA: Sierra Club Books.

Mann, J. (2014). Risk, religion, and invisibility. Journal of Appalachian Studies, $20(2), 124-131$

Mannion, G. \& Adey, C. (2011). Place-Based Education is an intergenerational practice. Children, Youth and Environments 21(1): 35-58.

Manzo, L. C. (2003). Beyond house and haven: Toward a revisioning of emotional relationships with places. Journal of Environmental Psychology 23, 47-61. DOI: $10.1016 / \mathrm{S} 0272-4944(02) 00074-9$

Manzo, L.C. (2005). For better or worse: Exploring multiple dimensions of place meaning. Journal of Environmental Psychology, 25, 67-86. DOI: 10.1016/j.jenvp.2005.01.002

Manzo, L.C. \& Devine-Wright, P. (Eds). (2014). Place attachment: Advances in theory, methods and applications. New York, NY: Routledge.

Manzo, L.C. \& Perkins, D. (2006). Finding common ground: The importance of place attachment to community participation in planning. Journal of Planning Literature, 20, 335-350. DOI: 10.1177/0885412205286160 
Marcus, G.E. \& Fischer, M.J. (1986). Anthropology as cultural critique: An experimental moment in the human sciences. Chicago, IL: University of Chicago Press.

Marshall, J.L., Thomas, L., Lane, N.M., Holmes, G.M., Arcury, T.A., Randolph, R... (2017). Health disparities in Appalachia. PDA, Inc. Cecil G. Sheps Center for Health Services Research. Appalachian Regional Commission. Retrieved from https://www.arc.gov/assets/research_reports/Health_Disparities_in_Appalachi a_August_2017.pdf

Martin, R. (2015). Coal Wars: The future of energy and the fate of the planet. New York, NY: Palgrave Macmillan.

Mason, R. (2011). Confronting uncertainty: Lessons from rural social work. Australian Social Work, 64, 377-394. DOI:10.1080/0312407X.2011.574144

Massey, D. (1993). Power-geometry and a progressive sense of place. In J. Bird, B. Curtis, T. Putnam, G. Robertson, \& L. Tickner (Eds.) Mapping the futures: local cultures, global change (pp.59-69). London, United Kingdom: Routledge.

Massey, D. (1994). Space, place, and gender. Minneapolis, MN: University of Minnesota Press.

Matthews, E.M. (1966). Neighbor and kind: Life in a Tennessee Ridge community. Nashville, TN: Vanderbilt University Press.

Mazumdar, S. \& Mazumdar, S. (2004). Religion and place attachment: A study of sacred places. Journal of Environmental Psychology, 24, 385-397. DOI: 10.1016/j.jenvp.2004.08.005

Mead, G.H. (1934). Mind, self and society: From the standpoint of a social behaviorist. Chicago, IL: University of Chicago Press. 
McCauley, D.V. (1995). Appalachian mountain religion: A history. Urbana, IL: University of Illinois Press.

McClay, W.M. (2014). Why place matters. In W.M. McClay, \& T.V. McAllister (Eds.) Why place matters: Geography, identity, and civic life in modern America (pp. 1-9). New York, NY: Encounter Books.

McClay, W.M. \& McAllister, T.V. (Eds.) (2014). Why place matters: Geography, identity, and civic life in modern America. Encounter Books: New York.

McCurdy, P. \& Uldam, J. (2014). Connecting participant observation positions: Toward a reflexive framework for studying social movements. Field Methods, 26(1), 40-55. DOI: $10.1177 / 1525822 X 13500448$

McCurry, A. L., Schrodt, P., \& Ledbetter, A. M. (2012). Relational uncertainty and communication efficacy as predictors of religious conversations in romantic relationships. Journal of Social and Personal Relationships, 29, 1085-1108, DOI: $10.1177 / 0265407512449402$

McDonald, N. C. (2015). Are Millennials really the "go-nowhere" generation? Journal of The American Planning Association, 81, 90-103.

DOI: $10.1080 / 01944363.2015 .1057196$

McGranahan, David A. \& Beale, C. (2002). Understanding rural population loss. Rural America, 17(4): 2-11.

McGreal, C. (November 12, 2016). America's poorest white town: Abandoned by coal, swallowed by drugs. The Guardian. Retrieved from: https://www.theguardian.com/us-news/2015/nov/12/beattyville-kentucky-andamericas-poorest-towns

McHugh, K. E. \& Mings, R. C. (1996). The circle of migration: Attachment to place in aging. Annals of the Association of American Geographers, 86, 530-550. 
McInerney, P., Smyth, J., \& Down, B. (2011). 'Coming to a place near you?' The politics and possibilities of a critical pedagogy of place-based education. AsiaPacific Journal of Teacher Education, 39, 3-16.

McLaughlin, D. K., Shoff, C. M., \& Demi, M. A. (2014). Influence of perceptions of current and future community on residential aspirations of rural youth. Rural Sociology, 79(4), 453-477. doi:10.1111/ruso.12044

McLean, K., Wood, B., \& Breen, A. (2013). Reflecting on a difficult life: Narrative construction in vulnerable adolescents. Journal of Adolescent Research, 28, 431-452. DOI:10.1177/0743558413484355

McMichael, P. (2014). The power of conversation. Journal of Appalachian Studies, $20(2), 142-145$.

McSpirit, S., \& Reid, C. (2011). Residents' Perceptions of Tap Water and Decisions to Purchase Bottled Water: A Survey Analysis from the Appalachian, Big Sandy Coal Mining Region of West Virginia. Society \& Natural Resources, 24(5), 511-520. DOI:10.1080/08941920903401432

Meit, M., Heffernan, M., Tanenbaum, E., \& Hoffmann, T. (2017). Appalachian diseases of despair. The Walsh Center for Rural Health Analysis. Retrieved from https://www.arc.gov/research/researchreportdetails.asp?REPORT_ID=139

Merton, R. K. (1972). Insiders and outsiders: a chapter in the sociology of knowledge. American Journal of Sociology, 78(1), 9-47. Retrieved from http://www.jstor.org/stable/2776569

Mesch, G.S. \& Manor, O. (1998). Social ties, environmental perception, and local attachment. Environment and Behavior, 30, 504-519. DOI: $10.1177 / 001391659803000405$ 
Milkman, R. (2017). A new political generation: Millennials and the post-2008 wave of protest. American Sociological Review, 82, 1-31.

DOI:10.1177/0003122416681031

Mills, J., Bonner, A., \& Francis, K. (2006). The development of constructivist grounded theory. International Journal of Qualitative Methods, 5, Article 3. Retrieved from: http://www.ualberta.ca/ iiqm/backissues/5_1/pdf/mills.pdf

Mitchell, M.T. (2014). Making places: The cosmopolitan temptation. In W.M.

McClay, \& T.V. McAllister. (Eds.), Why place matters: Geography, identity, and civic life in modern America (pp.84-101). Encounter Books: New York.

Moore, L. \& Savage, J. (2002). Participant observation, informed consent, and ethical approval. Nurse Researchers, 9, 58-70. DOI: 10.7748/nr2002.07.9.4.58.c6198

Moore, T.G. (1991). Economic development in Appalachian Kentucky, 1800-1860. In R.D. Mitchell (Ed.) Appalachian Frontiers (pp.222-34). Lexington, KY: University of Kentucky Press.

Morey, A. C., Eveland, W. P. Jr., \& Hutchens, M. J. (2012). The "who" matters: Types of interpersonal relationships and avoidance of political disagreement. Political Communication, 29(1): 86-103, DOI: $10.1080 / 10584609.2011 .641070$

Morrice, E., \& Colagiuri, R. (2013). Coal mining, social injustice and health: A universal conflict of power and priorities. Health \& Place, 19, 74-79. DOI:10.1016/j.healthplace.2012.10.006

Morrone, M., Kruse, N. A., \& Chadwick, A. E. (2014). Environmental and health disparities in Appalachian Ohio: Perceptions and Realities. Journal of Health Disparities Research \& Practice, 7(5), 67-81. 
Mountain Association for Community Economic Development. (n.d.). Five-year plan, 2016-2010. Retrieved from:https://maced.org/wp content/uploads/2018/05/MACED_5_Year_Plan.pdf

Mudliar, P. \& Arnold, G. (2017). Fighting king coal: The barriers to grassroots environmental justice movement participation in Central Appalachia. Review of Policy Research, 34(5), 727-729. DOI:10.1111/ropr.12263

Munford, R., \& Sanders, J. (2015). Negotiating and constructing identity: Social work with young people who experience adversity. British Journal of Social Work, 45, 1564-1580. DOI:10.1093/bjsw/bcu059

Murphy, D.W.A. (2015). Theorizing climate change, (im)mobility and socioecological systems resilience in low-elevation coastal zones. Climate and Development, 7(4), 380-397. DOI: 10.1080/17565529.2014.953904

National Association of Social Workers. (2009). Rural social work. Retrieved from https://www.socialworkers.org/assets/secured/documents/da/da2010/referred/ Rural\%20Social\%20work.pdf

Newheiser, A-K., Barreto, M., \& Tiemersma, J. (2017). People like me don't belong here: Identity concealment is associated with negative workplace experiences. Journal of Social Issues, 73, 341-348. DOI: 10.1111/josi.12220

Nespor, J. (2008). Education and place: A review essay. Educational Theory, 48(4), $475-489$.

Noble-Carr, D., \& Woodman, E. (2018). Considering identity and meaning constructions for vulnerable young people. Journal of Adolescent Research, 33(6), 672-698. DOI: 10.1177/0743558416684952 
Nord, W. R., Brief, A. P., Atieh, J. M., \& Doherty, E. M. (1990). Studying meanings of work: The case of work values. In A. Brief \& W. Nord (Eds.), Meanings of occupational work: A collection of essays. Lexington, MA: Lexington Books.

Nordström, M. (2014). Young people's attachment to place. In, Edgerton, E., Romice, O., \& Thwaites, K. (Eds). Bridging the boundaries: Human experience in the natural and built environment and implications for research, policy, and practice (pp.73-82). Advances in People-Environment Studies, Volume 5. Boston, MA: Hogrefe.

Oakley, A. (1981). Interviewing women: A contradiction in terms. In H. Roberts (Ed.). Doing feminist research (pp. 30-61). London, UK: Routledge \& Kegan Paul.

Obermiller, P.J. \& Howe, S.R. (2000). Appalachian migration patterns, 1975-1980 and 1985-1990. Appalachian Regional Commission, Research Reports. Retrieved from https://www.arc.gov/assets/research_reports/AppalachianMigrationPatterns.pd $\mathrm{f}$

Obermiller, P.J., Wagner, T.E., \& Tuckers, E.B. (Eds.) (2000). Appalachian odyssey: Historical perspectives on the great migration. Westport, CT: Praeger.

Padgett, D. K. (2008). Qualitative methods in social work research. ( $2^{\text {nd }}$ Ed.) Thousand Oaks, CA: Sage.

Partners for Education. (2017). Promise Neighborhood brings $\$ 30$ million to support education. Berea College. Retrieved from https://www.berea.edu/pfe/news/promise-neighborhood-brings-30-millionsupport-education/ 
Patterson, M.E. \& Williams, D.R. (2005). Maintaining research traditions on place: diversity of thought and scientific progress. Journal of Environmental Psychology, 25, 361-380. DOI: 10.1016/j.jenvp.2005.10.001

Payne, M. (2014). Modern Social Work Theory (4 ${ }^{\text {th }}$ Ed.). Chicago, IL: Lyceum Books, Inc.

Pearsall, M. (1959). Little Smokey Ridge: The natural history of a southern Appalachian neighborhood. University, AL: University of Alabama Press.

Peet, R. (1998). Modern geographic thought. Oxford: Blackwell.

Philliber, W.W. (1981). Appalachian migrants in urban America: Cultural conflict or ethnic group formation? New York, NY: Praeger.

Philliber, W. W. \& McCoy, C.B. (1981). (Eds.). The invisible minority: Urban Appalachians. Lexington, KY: University of Kentucky Press.

Phillips, A., Quinn, A., \& Hietkamp, T. (2010). Who wants to do rural social work? Student perceptions of rural social work practice. Contemporary Rural Social Work, 2, 51-65.

Piche, V. (2013). Contemporary migration theories as reflected in their founding texts. Population-E, 68(1), 141.164. DOI: 10.3917/pope.1301.0141. Retrieved from: https://pdfs.semanticscholar.org/3434/a6a534c9e06bf157e9feed5b237910455 51a.pdf

Pickles, J. (2018). Place, autonomy, and the politics of hope. In Billings, D.B. \& Kingsolver, A.E. (Eds.). Appalachia in regional context: Place matters (pp. 71-90). Lexington, KY: University Press of Kentucky. 
Pidgeon, N., \& Henwood, K. (1997). Using grounded theory in psychological research. In N. Hayes (Ed.) Doing qualitative analysis in psychology (pp. 245273). Hove, UK: Psychology Press.

Pitts, Jr., L. (2014, October 5). White poverty exists, ignored. Miami Herald. Retrieved from http://www.miamiherald.com/opinion/opn-columnsblogs/leonard-pitts-jr/article2518087.html

Pollard, K. (2003). Appalachia at the Millennium: An overview of results from Census 2000. Demographic and Socioeconomic Change in Appalachia. Population Reference Bureau. Retrieved from: https://assets.prb.org/pdf/AppalachiaMillenniumOvervie.pdf

Pollard, K. (2005). Population growth and distribution in Appalachia: New realities. Demographic and socioeconomic change in Appalachia. Retrieved from https://assets.prb.org/pdf05/PopGrowthAppalachia.pdf

Pollard, K. \& Jacobsen, L. (2018). The Appalachian Region: A data overview from the 2012-2016 American Community Survey. Population Reference Bureau. Retrieved from https://www.arc.gov/assets/research_reports/DataOverviewfrom2012to2016A CS.pdf

Poznick, B., Nash, C., Williams, J.P., Hevert, J., Stevens, J.... (Executive Produers). (2013-). Buckwild [Television series]. New York, New York: MTV.

Pratt, M. G., \& Ashforth, B. E. (2003). Fostering meaningfulness in working and at work. In K. S. Cameron, J. E. Dutton, \& R. E. Quinn (Eds.), Positive organizational scholarship (pp. 309-327). San Francisco: Berrett-Koehler Publishers, Inc. 
Precourt, W. (1983). The image of Appalachian poverty. In A. Batteau (Ed.), Appalachia and America: Autonomy and regional dependence (pp. 86-110). Lexington: University Press of Kentucky.

Pred, A. (1984). Place as historically contingent process: Structuration and the timegeography of becoming places. Annals of the Association of American Geographers, 74(2), 279-297. DOI: 10.1111/j.1467-8306.1984.tb01453.x

Prince, D. (2014). What about place? Considering the role of physical environment on youth imagining of future possible selves. Journal of Youth Studies, 17, 697716. DOI: 10.1080/13676261.2013.836591.

Prochansky, H.M., Fabian, A.K., \& Kaminoff, R. (1983). Place-identity: Physical world socialization of the self. Journal of Environmental Psychology, 3, 5783. DOI: $10.1016 / \mathrm{S} 0272-4944(83) 80021-8$

Puckett, A. (2000). Seldom ask, never tell: Labor and discourse in Appalachia. Oxford, UK: Oxford University Press.

Pugh, J., Mitchell, M., \& Brook, F. (2000). Insider/outsider partnerships in an ethnographic study of shared governance. Nursing Standard, 14(27), 43-44. DOI: $10.7748 / \mathrm{ns} 2000.03 .14 .27 .43 . c 2798$

Pugh, R. (2007). Dual relationships: Personal and professional boundaries in rural social work. British Journal of Social Work, 37, 1405-1423. DOI: 10.1093/bjsw/bc1088

Quinton, S. (November 18, 2016). Dedicated to the mountains, desperate for jobs. Stateline. Pew Charitable Trusts. Retrieved from http://www.pewtrusts.org/en/research-andanalysis/blogs/stateline/2016/11/18/dedicated-to-the-mountains-desperate-forjobs 
Ramalho, R., Adams, P., Huggard, P. \& Hoare, K. (2015). Literature review and constructivist grounded theory methodology. Forum: Qualitative Social Research, 16(3), Article 19. Retrieved from:

http://www.qualitativeresearch.net/index.php/fqs/article/view/2313/3876

Raymond, C.M., Brown, G. \& Weber, D. (2010). The measurement of place attachment: Personal, community, and environmental connections. Journal of Environmental Psychology 30(4):422-34.

Reid, H.G. \& Taylor, B. (2010). Recovering the commons: Democracy, place, and global justice. Urbana, IL: University of Illinois Press.

[Residents of Owsley County/PRCT TV]. (2018, March 25). Homesong II 2014. [Video file]. Retrieved from https://www.youtube.com/watch?v=7A284tN9f1g Relph, E. (1976). Place and placelessness. London: Pion.

Relph, E. (2009). A pragmatic sense of place. Environmental and Architectural Phenomenology, 20, 24-31.

Rev.com, Inc. (2018). Rev Voice Recorder (C. Retrieved from: https://itunes.apple.com/us/app/revvoicerecorder/id598332111?mt=8\&\&referr er $=$ click\%3Dabc61083-9e8c-4798-966f-4136bc107601

Rice, J. L. \& Burke, B. J. (2018). Building more inclusive solidarities for socioenvironmental change: Lessons in resistance from Southern Appalachia. Antipode, 50(1), 212-232. DOI:10.1111/anti.12336

Rickett, J. (February 3, 2017). Fulfilling the Promise Zone in eight Kentucky counties. The Lexington Herald-Leader. Retrieved from https://www.kentucky.com/opinion/op-ed/article130625674.html 
Riebschleger, J. (2007). Social worker's suggestions for effective rural practice. Families in Society, 88(2), 203-213. DOI: 10.1606/1044-3894.3618

Ritzer, G. (2011). Sociological Theory ( $8^{\text {th }}$ Ed.). New York, NY: McGraw-Hill. Robson, D. \& Evans, G. (Eds). (2010). Towards re-enchantment: Place and its meanings. London: Artevents.

Rodriguez, A. (Ed.). (2008). The multiple faces of agency: Innovative strategies for effecting change in urban school contexts. Rotterdam, the Netherlands: Sense Publishers.

Rogers, Z. (July 10, 2018). Anti-poverty activists finally allowed to enter the Kentucky capitol. The Courier Journal. Retrieved from: https://www.courierjournal.com/story/news/politics/2018/07/10/poor-peoples-campaign-allowedinto-kentucky-capitol/771176002/

Rosenbaum, T. Y. (2009). Applying theories of social exchange and symbolic interaction in the treatment of unconsummated marriage/relationship. Sexual \& Relationship Therapy, 24, 38-46. DOI:10.1080/14681990902718096

Rosso, B. D., Dekas, K. H., \& Wrzesniewski, A. (2010). On the meaning of work: A theoretical integration and review. Research in Organizational Behavior, 30, 91-127. DOI:10.1016/j.riob.2010.09.001

Rubin, A. \& Babbie, E. (2016). Essential research methods for social work (4 ${ }^{\text {th }}$ Ed.). Australia: Cenage Learning.

Rubin, H.J. \& Rubin, I.S. (2012). Qualitative interviewing: The art of hearing data. ( $3^{\text {rd }}$ Ed.) Los Angeles, CA: Sage.

Russell, A. (2011). A grounded theory of liberated identity: Lesbians transcending oppression. The Grounded Theory Review: An International Journal, 10(1), 59-83.) 
Russell, J. \& Ward, L.M. (1982). Environmental psychology. Annual Review of Psychology, 33, 651-688. DOI: 10.1146/annurev.ps.33.020182.003251

Rybczyski, W. (2014). The demand side of urbanism. In W.M. McClay, \& T.V. McAllister. (Eds.), Why place matters: Geography, identity, and civic life in modern America (pp.120-148). Encounter Books: New York.

Ryerson, S. \& Schept, J. (April 28, 2018). Building prisons in Appalachia: The region deserves better. Boston Review. Retrieved from: http://bostonreview.net/lawjustice/sylvia-ryerson-judah-schept-building-prisons-appalachia

Saar, M., \& Palang, H. (2009). The dimensions of place meanings. Living Reviews in Landscape Research, 3, 1-24. DOI: 10.12942/lrlr-2009-3

Sachs, J. D. (2015). The Age of Sustainable Development. New York, NY: Columbia University press.

Sack, R. (1983). Human territoriality: A theory. Annals of the Association of American Geographers, 73, 55-74. DOI:10.1111/j.1467-8306.1983.tb01396.x

Sack, R. D. (1986). Human territoriality: Its theory and history. Cambridge, UK: Cambridge University Press.

Sagoff, M. (1988). The economy of the Earth: Philosophy, law and the environment. Cambridge, UK: Cambridge University Press.

Salmon, A. (2007). Walking the talk: How participatory interview methods can democratize research. Qualitative Health Research, 17, 982-994. DOI: $10.1177 / 1049732307305250$

Salstrom, P. (1994). Appalachia's path to dependency: Rethinking a region's economic history, 1730-1940. Lexington, KY: The University Press of Kentucky. 
Salstrom, P. (2004). The Great Depression. In R.A. Straw \& H.T. Blethen (Eds.) High mountains rising: Appalachia in time and place (pp. 74-87). Urbana, IL: University of Illinois Press.

Scales, T. L., Streeter, C. L., \& Cooper, H. S. (Eds.), Rural Social Work: Building and Sustaining Community Capacity (2nd Ed). Hoboken, NJ: Wiley \& Sons.

Schambra, W.A. (2014). Place and poverty. In W.M. McClay, \& T.V.McAllister. (Eds.), Why place matters: Geography, identity, and civic life in modern America (pp.162-170). Encounter Books: New York.

Scherch, J. (2015). Rural community transition and resilience: What now for social work? Contemporary Rural Social Work, 7, 56-70. Retrieved from http://journal.minotstateu.edu/crsw/article/view/715

Schoenberg, N., Bundy, H., Baeker Bispo, J., Studts, C., Shelton, B., \& Fields, N. (2015). A Rural Appalachian faith-placed smoking cessation intervention. Journal of Religion \& Health, 54(2), 598-611.

Schrimshaw, E. W., Downing, M. J. Jr, Cohn, D. J., \& Siegel, K. (2014). Conceptions of privacy and the non-disclosure of same-sex behaviour by behaviourallybisexual men in heterosexual relationships. Culture, health \& sexuality, 16, 351-365, DOI: 10.1080/13691058.2014.887779

Schwandt, T.A. \& Halpern, E.S. (1988). Linking auditing and meta-evaluation: Enhancing quality in applied research. Newbury Park, CA: Sage.

Schwartz, S. J., Zamboanga, B. L., Luyckx, K., Meca, A., \& Ritchie, R. A. (2013). Identity in emerging adulthood reviewing the field and looking forward. Emerging Adulthood, 1, 96-113. doi:10.1177/2167696813479781 
Schwarzweller, H.K., Brown, J.S., \& Mangalam, J.J. (1979). Transition: A case study of Appalachian migration. University Park, PA and London, UK: The Pennsylvania State University Press.

Seamon, D. (2014). Place attachment in phenomenology: The synergistic dynamism of place. In Manzo, L.C. \& Devine-Wright, P. (Eds). Place attachment: Advances in theory, methods and application (pp. 11-22). New York: Routledge.

Seidman, I.E. (1991). Interviewing as qualitative research: A guide for researchers in education and the social sciences. New York, NY: Teachers College, Columbia University.

Semken, S. (2005). Sense of place and place-based introductory geoscience teaching for American Indian and Alaska Native undergraduates. Journal of Geoscience Education, 53, 149-157.

Shamai, S. (1991). Sense of place: An empirical measurement. Geoforum, 22, 34758.

Shaping Our Appalachian Region. (2016). 2016 Impact Report. Retrieved from http://www.soarky.org/sites/default/files/Documentation/SOAR\%20Impact $\% 20$ Report $\% 20$ Fin al.pdf

Shapiro, H.D. (1977). Appalachia and the idea of America: The problem of the persisting frontier. In L. Brown L \& J. Williamson (Eds.) An Appalachian Symposium: Essays Written in Honor of Cratis D. Williams (pp. 43-55). Boone, NC: Appalachian State University. Retrieved from http://www.jstor.org/stable/j.ctt1xp3mkm.8 
Shapiro, H.D. (1978). Appalachia on our mind: The southern mountains and mountaineers in the American consciousness, 1870-1920. Chapel Hill, NC: University of North Carolina Press.

Shaw, I. (2008). Ethics and the practice of qualitative research. Qualitative Social Work, 7, 400-414. DOI: 10.1177/1473325008097137

Shelby, A. (2014, August 1). Speak your piece: 'The hardest place to live.' The Daily Yonder: Keep it rural. Retrieved from: http://www.dailyyonder.com/speakyour-piece-hardest-place-live/2014/07/31/7476

Shen, S. \& Gemenne, F. (2011). Contrasted views on environmental change and migration: The case of Tuvaluan migration to New Zealand. International Migration, 49(S1), e225-e242. DOI:10.1111/j.1468-2435.2010.00635.x

Shumway, M.J., Otterstrom, S., \& Glavac, S. (2014). Environmental hazards as disamenities: Selective migration and income change in the United States from 2000-2010. Annals of the Association of American Geographers, 104, 280-291. DOI: 10.1080/00045608.2013.873322

Silverman, D. \& Marvasti, A. (2008). Doing qualitative research: A comprehensive guide. Los Angeles, CA: Sage.

Simon, R. M. (1983-4). Regions and social relations: A research note. Appalachian Journal

24, 22-31. Retrieved from http://www.jstor.org/stable/40932554

Simpson, A.R. (2008). Changes in young adulthood: Young Adulthood. Massachusetts Institute of Technology, Young Adult Development Project. Retrieved from: http://hrweb.mit.edu/worklife/youngadult/changes_ya.html

Slovak, K., Sparks, A. \& Hall, S. (2011). Attention to rural populations in social work's scholarly journals. Journal of Social Service Research, 37, 428-438. 
DOI: $10.1080 / 01488376.2011 .578035$

Smith, A. (2016). Appalachian Futurism. Journal of Appalachian Studies, 22(1), 7375. DOI:10.5406/jappastud.22.1.0073

Smith, B.E. (2018). Transforming places: Toward a global politics of Appalachia. In D.B. Billings \& A.E. Kingsolver (Eds.). Appalachia in regional context: Place matters (pp.49-70). Lexington, KY: University Press of Kentucky.

Smith, B.E. \& Fisher, S. (2016). Reinventing the region: Defining, theorizing, organizing Appalachia. Journal of Appalachian Studies, 22(1), 76-79. DOI:10.5406/jappastud.22.1.0076

Smith, G. A. (2002). Place-based education: Learning to be where we are. Phi Delta Kappan, 83, 584-594.

Smith, G. A. (2007). Place-based education: Breaking through the constraining regularities of public school. Environmental Education Research, 13(2): 17188.

Smith, G.A., \& Sobel, D. (2010). Place and community-based education in schools. London: Routledge.

Smith, M. (2005). On 'being' moved by nature: Geography, emotion and environmental ethics. In Davidson, J., Bondi, L., \& Smith, M. (Eds.), Emotional Geographies, (pp. 219-230). New York, NY: Routledge.

Smith, M., Davidson, J., Cameron, L., \& Bondi, L. (Eds.) (2009). Emotion, place and culture. Burlington, VT: Ashgate.

Sobel, D. (2004). Place-based education: Connecting classroom and community. Great Barrington, MA: The Orion Society. 
Sociocultural Research Consultants, LLC. (2014). Dedoose, version 5.0.11 [Web application]. Los Angeles, CA: About. Accessed [July 4, 2016] from: http://www.dedoose.com

Soobryan, V. (2003). Ethics, truth, and politics in constructivist qualitative research. Westminster Studies in Education, 26, 107-123. DOI: $10.1080 / 0140672030260204$

Sovine, M.L. (1983). Studying religious belief systems in their social historical context. In, Batteau, A. (Ed). Appalachia and America: Autonomy and regional dependence (pp. 48-67). Lexington, KY: University of Kentucky Press.

Sparks, J. R., \& Schenk, J. A. (2001). Explaining the effects of transformational leadership: An investigation of the effects of higher-order motives in multilevel marketing organizations. Journal of Organizational Behavior, 22, 849-869. DOI:10.1002/job.116

Spradley, J.P. (1980). Participant Observation. Long Grove, IL: Waveland Press, Inc.

Strauss, A. \& Corbin, J. (1990). Basics of qualitative research: Grounded theory procedures and techniques. Newbury Park, CA: Sage.

Stay Together Appalachian Youth. (2018). August 2018 Newsleeter. Retrieved from: https://www.thestayproject.com/blog-stay-in-the-news

Stedman, R. C., Jacquet, J.B., Filteau, M.R., Willits, F.K., Brasier, K.J., \& McLaughlin, D.K. (2012). Marcellus shale gas development and new boomtown research: Views of New York and Pennsylvania residents. Environmental Practice, 14, 382-92. DOI: 10.1017/S1466046612000403

Steger, M.F., Dik, B.J., \& Duffy, R.D. (2012). Measuring meaningful work: The work and meaning inventory (WAMI). Journal of Career Assessment, 20, 1-16. 
DOI: $10.1177 / 1069072711436160$ Retrieved from: DOI:

$10.1177 / 1069072711436160$

Stephens, H. M., Partridge, M. D., \& Faggian, A. (2013). Innovation, entrepreneurship and economic growth in lagging regions. Journal of Regional Science, 53(5), 778-812. DOI: 10.1111/jors.12019

Stephenson, J. (1968). Shiloh: A mountain community. Lexington, KY: University Press of Kentucky.

Stein, J. (2013). Millennials: The me me me generation. TIME, U.S. [May 20, 2013]. http://time.com/247/millennials-the-me-me-me-generation/

Stevenson, R.B. (2008). A critical pedagogy of place and the critical place(s) of pedagogy. Education Research, 14, 353-360.

Stone, L. (2016, June 2). A brief population history of Central Appalachia, they peaked in 1830. In a State of Migration. Retrieved from https://medium.com/migration-issues/a-brief-population-history-of-centralappalachia-b65a58bc6e6c

Stryker, B. P.J. (2000). The past, present and future of an identity theory. Social Psychology Quarterly, 63, 284-297.

Stuart, P.H. (2014). Social welfare and rural people: From the colonial era to the present. In T.L. Scales, C.L. Streeter, \& H.S. Coopers (Eds.). Rural social work: Building and sustaining community capacity ( $2^{\text {nd }}$ Ed., pp. 29-44). Hoboken, NJ: Wiley \& Sons.

Styles, J. (1979). Outsider/insider: researching gay baths. Urban Life, 8, 135-52. DOI: $10.1177 / 089124167900800202$

Tanenhaus, S. (August 15, 2014). Generation nice. The New York Times. Retrieved from: 
https://www.nytimes.com/2014/08/17/fashion/the-millennials-are-generationnice.html?_r=0

Taylor, C.C. \& Townsend, A. R. (1976). The local 'sense of place' as evidenced in North-East England. Urban Studies, 13, 133-146. Retrieved from http://www.jstor.org/stable/43081514

Taylor, P. \& Keeter, S. (2010). (Eds). Millennials: Confident. Connected. Open to change. The Pew Research Center. Retrieved from:

http://assets.pewresearch.org/wp-content/uploads/sites/3/2010/10/millennialsconfident-connected-open-to-change.pdf

TelTech. (2018). TapeACallC. Retrieved from: https://www.tapeacall.com

Therborn, G. (2006). Why and how place matters. In R. E. Goodin \& C. Tilly (Eds.) The Oxford handbook of contextual political analysis (pp. 509-533). Oxford: Oxford University Press.

Thompson, D. (April 26, 2013). The unluckiest generation: What will become of Millennials? The Atlantic. Retrieved from:

https://www.theatlantic.com/business/archive/2013/04/the-unluckiestgeneration-what-will-become-of-millennials/275336/

Timseena, B. (2009). Participant observation in field research: An overview. Nepalese Journal of Qualitative Research Methods, 3, 75-86.

Toth, G. (2014). Place-conscious transportation and policy. In W.M. McClay, \& T.V. McAllister. (Eds.), Why place matters: Geography, identity, and civic life in modern America (pp.48-58). New York, NY: Encounter Books.

Towers, G. (2007). West Virginia's lost youth: Appalachian stereotypes and residential preferences. Journal of Geography, 104, 74-84. DOI: 10.1080/00221340508978619?journalCode=rjog20 
Trentleman, C.K. (2009). Place attachment and community attachment: A primer grounded in the lived experience of a community sociologist. Society and Natural Resources, 22, 191-210. DOI: 10.1080/08941920802191712

Tuan, Y. F. (1977). Space and place: The perspective of experience. Minneapolis, MN: University of Minnesota Press.

Tuckett, A.G. (2005). Part II. Rigor in qualitative research: Complexities and solutions. Nurse Researcher, 13, 29-42. DOI: $10.7748 / \mathrm{nr} 2005.07 .13 .1 .29 \mathrm{c} 5998$

Turner, J.H. (2011). Extending the Symbolic Interactionist Theory of Interaction Processes: A Conceptual Outline. Symbolic Interaction, 34(3), 330-339. DOI:10.1525/si.2011.34.3.330

Turpin, A. J. (2015). Higher Ground 5: Find a Way. Journal Of Appalachian Studies, 21(2), 292-295.

Twenge, J.M. \& Campbell, K.W. (2010). The narcissism epidemic: Living in the age of entitlement. New York, NY: Atria.

Twigger-Ross, C., Bonaiuto, M., \& Breakwell, G. (2003). Identity theories and environmental psychology. In, M. Bonnes, T. Lee, M. Bonaiuto (Eds.), Psychological theories for environmental issues (pp. 203-234). Ashgate Publishing Company, Hants

United Nations. (2014). World urbanization prospects: The 2014 Revision, Highlights. Department of Economic and Social Affairs, Population Division. Retrieved from: http://esa.un.org/unpd/wup/Highlights/WUP2014Highlights.pdf 
United States Department of Agriculture. (2017). Rural-urban continuum codes.

Economic Research Service. Retrieved from: https://www.ers.usda.gov/dataproducts/rural-urban-continuum-codes/documentation/

United States Forest Service. (n.d.). Tri-State Floods, January 29-30, 1957: Disaster through land misuse. Berea, KY: Special Collections and Archives Hutchins Library, Berea College.

United States Office of Rural Health Policy. (2016). List of rural counties and designated eligible Census tracts in metropolitan counties: 2010 Census. Retrieved from https://www.hrsa.gov/sites/default/files/ruralhealth/resources/forhpeligiblearea s.pdf

Valentine, G. (2001). At the drawing board: developing a research design. In M. Limb \& C. Dwyer (Eds.). Qualitative methodologies for geographers: Issues and debates (pp.41-54). Oxford University Press, Inc. New York, New York.

Vance, J.D. (2016). Hillbilly Elegy. New York, NY: HarperCollins.

Vargas-Cetina, G. (Ed.) (2013). Anthropology and the politics of representation. Tuscaloosa, AL: University of Alabama Press.

Vaske, J.J. \& Kobrin, K.C. (2001). Place attachment and environmentally responsible behavior. Journal of Environmental Psychology, 32, 116-121. DOI: $10.1080 / 00958960109598658$

Vespa, J. (2016). The changing economics and demographics of young adulthood: 1975-2016. U.S. Census Bureau. Population Characteristics. Retrieved from; https:/www.census.gov/content/dam/Census/library/publications/2017/demo/ p20-579.pdf 
Vidic, R. D., Brantley, S. L., Vandenbossche, J. M., Yoxtheimer, D., \& Abad, J. D. (2013). Impact of shale gas development on regional water quality. Science, 340 (6134): 826-35. DOI: 10.1126/science.1235009

Walls, D. S. (1978). Internal colony or internal periphery? A critique of current models and an alternative formulation. In H. Lewis, L. Johnson, \& D. Adkins (Eds.) Colonialism in modern America: The Appalachian case (pp. 319-349). Boone, NC: Appalachian Consortium Press.

Waples, D. A. (2012). The natural gas industry in Appalachia: A history from the first Discovery to the tapping of the Marcellus shale. Jefferson, NC: McFarland.

Waterman, A. S. (1982). Identity development from adolescence to adulthood: An extension of theory and a review of research. Developmental Psychology, 18, $341-358$.

Watts, J. (2011). Ethical and practical challenges of participant observation in sensitive health research. International Journal of Social Research Methodology, 14, 301-312. DOI: 10.1080/13645579.2010.517658

Webb-Sunderhaus, S. (2016). "Keep the Appalachian, drop the redneck": Tellable student narratives of Appalachian identity. College English, 79(1), 11-33.

Weick, K. E. (1995). Sensemaking in organizations. Thousand Oaks, CA: Sage. Weinbaum, E.S. (2004). To move a mountain: Fighting the global economy in Appalachia. New York, NY: The New Press.

Welch, W. (2011). Self Control, Fatalism, and Health in Appalachia. Journal of Appalachian Studies, 17(1/2), 108-122.

Weller, J. E. (1965). Yesterday's People: Life in Contemporary Appalachia. Lexington, KY: University Press of Kentucky. 
Wengraf, T. (2001). Qualitative research interviewing: Biographic narrative and semi-structured methods. London, UK: Sage.

White, J. M. (2014). Whiteman v. Chesapeake: Damage to human health and the environment as seen through an application to hydraulic fracturing. Ecology Law Quarterly, 41(2), 645-652. Retrieved from: http://scholarship.law.berkeley.edu/elq/vol41/iss2/24

Whyte, W.F. (1979). On making the most of participant observation. American Sociologist, 14(1), 56-66. Retrieved from: http://www.jstor.org/stable/27702360

Williams, D. R. (2008). Pluralities of place: A user's guide to place concepts, theories, and philosophies in natural resource management. In L.E. Kruger, T. Hall, E. Troy, \& M.C. (Eds.) Understanding concepts of place in recreation research and management, (pp.7-30). Gen. Tech. Rep. PNW-GTR-744. Portland, OR: U.S. Department of Agriculture, Forest Service, Pacific Northwest Research Station. Retrieved from: https://www.fs.usda.gov/treesearch/pubs/30668

Williams, K. (February 24, 2016). Prisons of Appalachia: Kentucky town hopes new facility can boost economy. Al Jazeera. Retrieved from: http://america.aljazeera.com/articles/2016/2/24/prison-town-appalachiakentucky-coal.html

Williams, D.R. \& Vaske, J.J. (2003). The measurement of place attachment: Validity and generalizability of a psychometric approach. Forest Science, 49(6): 830 840. Retrieved from: https://www.fs.usda.gov/treesearch/pubs/23746

Williams, J.A. (2002). Appalachia: A history. Chapel Hill, NC: The University of North Carolina Press. 
Wilson, S.H. (1990). Window on the mountains: Berea's Appalachia, 1870-1930. Filson Club Historical Quarterly, 64, 384-400.

Wojan, T. R. \& Nichols, B. (2018). Design, innovation, and rural creative places: Are the arts the cherry on top, or the secret sauce? PLoS ONE, 13(2), 1-23. DOI: 10.1371/journal.pone.0192962

Wolcott, H.F. (2001). Writing up qualitative research $\left(2^{\text {nd }}\right.$ Ed). Thousand Oaks, CA: Sage.

Wolfe, A. W., Black, L. W., Munz, S., \& Okamoto, K. (2017). (Dis)Engagement and everyday democracy in stigmatized places: Addressing brain drain in the rural United States. Western Journal of Communication, 81(2), 168-187.

DOI:10.1080/10570314.2016.1236980

Wolfram, W. \& Christian, D. (1976). Appalachian speech. Arlington, VA: Center for Applied Linguistics.

Wright, L. (2018). Evaluating place in orientations of narratives of internal migration. Narrative Inquiry, 28, 198-214. DOI: 10.1075/ni.17034.wri

Wrzesniewski, A., Dutton, J. E., \& Debebe, G. (2003). Interpersonal sensemaking and the meaning of work. In Kramer, R. M., \& Staw, B. M. Eds. Research in Organizational Behavior, 25, 93-135. DOI: 10.1016/S0191-3085(03)25003-6

Wright, W. (April 20, 2018). A year after white nationalist rally, he's leading a Black Student Union in Pikeville. The Lexington Herald-Leader. Retrieved from: https://www.kentucky.com/news/state/article209459174.html

Young Appalachian Leaders and Learners. (2018). Y'ALL statement regarding the J.D. Vance roundtable at the Appalachian Studies Association Annual Meeting. Retrieved from: https://academeblog.org/2018/04/23/yall-statement- 
regarding-the-j-d-vance-roundtable-at-the-appalachian-studies-associationannual-meeting/

Zahle, J. (2017). Privacy, informed consent, and participant observation. Perspectives on Science, 25, 465-487. DOI: 10.1162/POSC_a_00250

Zimmerman, K.J., Smith, J.M., Simonson, K., \& Myers, W.B. (2015). Familial relationship outcomes of coming out as an Atheist. Secularism and Nonreligion, 4(1), part 4. DOI: 10.5334/snr.aw

Zullig, K.J. \& Hendryx, M. (2011). Health-related quality of life among Central Appalachian residents in mountaintop mining counties. American Journal of Public Health, 101(5), 848-853. DOI: 10.2105/AJPH.2010.300073 


\title{
APPENDIX A
}

\section{INSTITUTIONAL REVIEW BOARD APPROVAL LETTERS}

\section{UNIVERSITY OE LOUISVILLE}

DATE:

TO:

FROM:

IRB NUMBER:

STUDY TITLE:

REFERENCE \#:

IRB STAFF CONTACT:
Human Subjects Protection Program Office

MedCenter One - Suite 200

501 E. Broadway

Louisville, KY 40202-1798

Office: 502.852.5188 Fax: 502.852.2164

\author{
November 27, 2017 (Corrected on November 27, 2017) \\ Thomas R Lawson, Ph.D. \\ The University of Louisville Institutional Review Board \\ 17.1039 \\ A qualitative study of millennial resistance to outmigration in Appalachian Kentucky \\ 648858 \\ Jackie Powell, CIP \\ $852-4101$
}

This study was reviewed on $11 / 21 / 2017$ by the Chair of the Institutional Review Board and approved through the Expedited Review Procedure, according to 45 CFR 46.110(b), since this study falls under Category 7: Research on individual or group characteristics or behavior (including, but not limited to, research on perception, cognition, motivation, identity, language, communication, cultural beliefs or practices, and social behavior) or research employing survey, interview, oral history, focus group, program evaluation, human factors evaluation, or quality assurance methodologies

This study was also approved through 45 CFR 46.116 (D), which means that it has been granted a waiver of informed consent because it meets the following criteria: (For Observational Data)

- $\quad$ The research involves no more than minimal risk to the subjects.

- The waiver or alteration will not adversely affect the rights and welfare of the subjects.

- $\quad$ The research could not practicably be carried out without the waiver or alteration.

- Whenever appropriate, the subjects will be provided with the additional pertinent information after participation.

This study was also approved through 45 CFR 46.116 (C), which means that an IRB may waive the requirement for the investigator to obtain a signed informed consent form for some or all subjects if it finds either: (For Interview data collection)

- $\quad$ That the only record linking the subject and the research would be the consent document and the principal risk would be potential harm resulting from a breach of confidentiality. Each subject will be asked whether the subject wants documentation linking the subject with the research, and the subject's wishes will govern; or

- That the research presents no more than minimal risk of harm to subjects and involves no procedures for which written consent is normally required outside of the research context.

The following items have been approved:

\begin{tabular}{|l|l|l|l|}
\hline Submission Components & Version & Outcome \\
\hline Form Name & Version 1.1 & Approved as Submitted \\
\hline Submit for Initial Review & Version 1.2 & Approved as Submitted \\
\hline IRB Study Application & Version 1.0 & $11 / 13 / 2017$ & Approved \\
\hline \multicolumn{4}{|l|}{}
\end{tabular}




\begin{tabular}{|l|l|l|l|}
\hline $\begin{array}{l}\text { Interview Log and Inclusion Criteria Tracking } \\
\text { Sheet }\end{array}$ & Version 1.0 & $10 / 31 / 2017$ & Approved \\
\hline Email Script & Version 1.0 & $10 / 02 / 2017$ & Approved \\
\hline Protocol & Version 1.3 & $11 / 13 / 2017$ & Approved \\
\hline Stay Consent Preamble & Version 1.3 & $11 / 13 / 2017$ & Approved \\
\hline
\end{tabular}

This study now has final IRB approval from 11/21/2017 through 11/20/2018.

For guidance on using iRIS, including finding your approved stamped documents, please follow the instructions at https://louisville.edu/research/humansubjects/iRISSubmissionManual.pdf

\section{Site Approval}

If this study will take place at an affiliated research institution, such as KentuckyOne Health, Norton Healthcare or University of Louisville Hospital, permission to use the site of the affiliated institution is necessary before the research may begin. If this study will take place outside of the University of Louisville Campuses, permission from the organization must be obtained before the research may begin (e.g. Jefferson County Public Schools). Failure to obtain this permission may result in a delay in the start of your research.

\section{Privacy \& Encryption Statement}

The University of Louisville's Privacy and Encryption Policy requires such information as identifiable medical and health records: credit card, bank account and other personal financial information; social security numbers; proprietary research data; dates of birth (when combined with name, address and/or phone numbers) to be encrypted. For additional information: http://security.louisville.edu/PolStds/ISO/PS018.htm.

Implementation of Changes to Previously Approved Research

Prior to the implementation of any changes in the approved research, the investigator will submit any modifications to the IRB and await approval before implementing the changes, unless the change is being made to ensure the safety and welfare of the subjects enrolled in the research. If such occurs, a Protocol Deviation/Violation should be submitted within five days of the occurrence indicating what safety measures were taken, along with an amendment to revise the protocol.

\section{Unanticipated Problems Involving Risks to Subjects or Others (UPIRTSOs)}

In general, these may include any incident, experience, or outcome, which has been associated with an unexpected event(s), related or possibly related to participation in the research, and suggests that the research places subjects or others at a greater risk of harm than was previously known or suspected. UPIRTSOs may or may not require suspension of the research. Each incident is evaluated on a case by case basis to make this determination. The IRB may require remedial action or education as deemed necessary for the investigator or any other key personnel. The investigator is responsible for reporting UPIRTSOs to the IRB within 5 working days. Use the UPIRTSO form located within the iRIS system to report any UPIRTSOs.

\section{Continuation Review Requirements}

You are responsible for submitting a continuation review 30 days prior to the expiration date of your research study. Investigators who allow their study approval to expire have committed significant non-compliance with federal regulations. Such lapses may require reporting to federal agencies, a program audit by compliance auditors to ensure that subjects were not enrolled during the expired period, and may lead to findings of serious and continuing noncompliance if expiration were to occur a second time.

Full Accreditation since June 2005 by the Association for the Accreditation of Human Research Protection Programs, Inc.

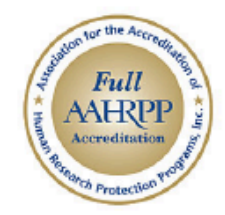


1099 Information (If Applicable)

As a reminder, in compliance with University policies and Internal Revenue Service code, all payments (including checks, pre-paid cards, and gift certificates) to research subjects must be reported to the University Controller's Office. Petty Cash payments must also be monitored by the issuing department and reported to the Controller's Office. Before issuing compensation, each research subject must complete a W-9 form. For additional information, please contact the Controller's Office at 852-8237 or controll@louisville.edu

The committee will be advised of this action at a regularly scheduled meeting.

If you have any questions, please contact the IRB analyst listed above or the Human Subjects Protection Program office at hsppofc@louisville.edu.

We value your feedback. Please let us know how you think we are doing: https://www.surveymonkey.com/r/CCLHXRP

Pat $m$ ansach

Peter M. Quesada, Ph.D., Chair

Social/Behavioral/Educational Institutional Review Board

$\mathrm{PMO} / \mathrm{jpp}$ 


\section{univensistry of LOUISVILLE}

DATE:

TO:

IRB NUMBER:

STUDY TITLE:

REFERENCE \#:

IRB STAFF CONTACT:
Human Subjects Protection Program Office

MedCenter One - Suite 200

$501 \mathrm{E}$. Broadway

Louisville, KY 40202-1798

Office: 502.852.5188 Fax: 502.852.2164

The amendment request has been received by the Human Subjects Protection Program Office and approved by the Vice Chair of the Institutional Review Board (IRB) on 08/03/2018 through the expedited review procedure according to 45 CFR 46.110(B). The following documents have been reviewed and approved:

\begin{tabular}{|l|l|l|l|}
\hline \multicolumn{2}{|l|}{ Submission Components } & Outcome \\
\hline Form Name & Version & Approved as Submitted \\
\hline $\begin{array}{l}\text { Request Application, Protocol, } \\
\text { or Document Change }\end{array}$ & Version 1.0 & \\
\hline \multicolumn{3}{|l|}{} \\
\hline Study Document & Version Date & Outcome \\
\hline Title & $08 / 01 / 2018$ & Approved \\
\hline $\begin{array}{l}\text { Changes to } \\
\text { recruitment log with } \\
\text { changes underlined }\end{array}$ & Version 2.0 & & \\
\hline Revised protocol & Version 2.0 & $08 / 01 / 2018$ & Approved \\
\hline
\end{tabular}

The modifications include: We would like to add the collection of race, gender, and age to the demographic information provided by participants. Participants will be asked to provide this on their own terms, however they identify. Collection of this data will occur during initial interviews or follow up contact with previous participants. It will be initially recorded on the recruitment log, noted by participant identification number in a password protected file in Dropbox. After recording in Dropbox, the recruitment log will be blacked out and shredded continuously.

The committee will be advised of this action at a regularly scheduled meeting.

For guidance on using iRIS, including finding your approved stamped documents, please follow the instructions at http://louisville.edu/research/humansubjects/iRISSubmissionManual.pdf.

If you have any questions, please contact the IRB analyst listed above or the Human Subjects Protection Program office at hsppofc@louisville.edu.

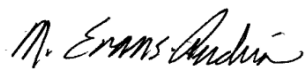

Melissa Evans Andris, PhD, Vice Chair

Social/Behavioral/Education Institutional Review Board 


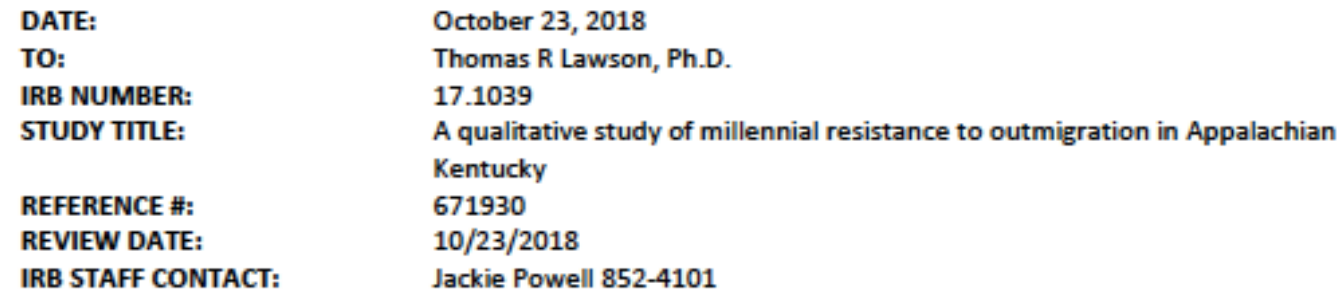

Jackie Powell 852-4101

The continuation request for this study was reviewed by the Chair of the Institutional Review Board (IRB) through the expedited review procedure, according to 45 CFR 46.110 and 21 CFR 56.110 since this study falls under Category 7: Research on individual or group characteristics or behavior (including, but not limited to, research on perception, cognition, motivation, identity, language, communication, cultural beliefs or practices, and social behavior) or research employing survey, interview, oral history, focus group, program evaluation, human factors evaluation, or quality assurance methodologies .

The study now has continued committee approval from 11/21/2018 through 11/20/2019.

The following items were reviewed and approved:

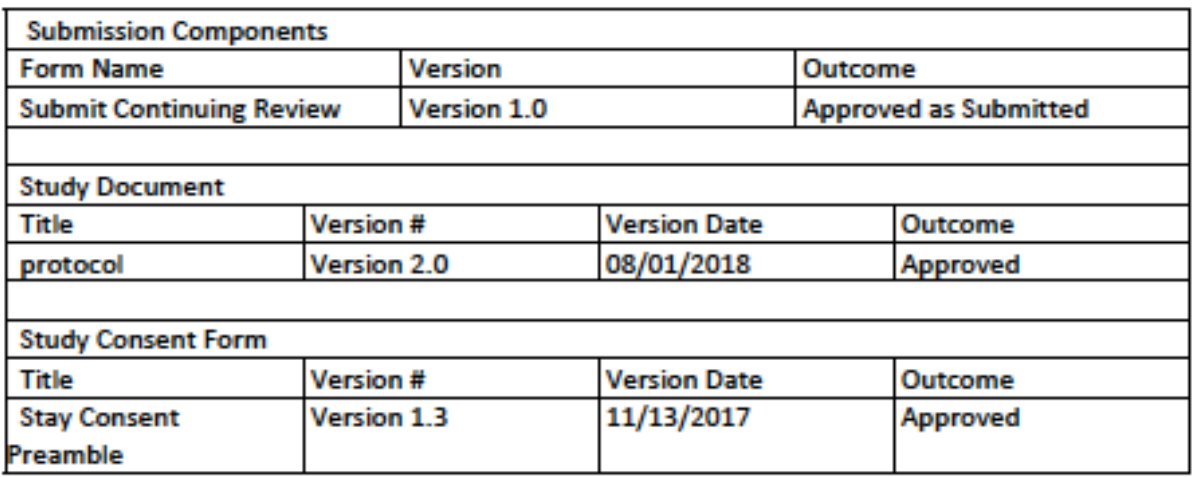

Please begin using your newly approved (stamped) consent document(s) on 11/21/2018. The previous version(s) will no longer be valid.

* Please submit an amendment to increase the number of subjects you wish to enroll. The amendment will affect the iRIS application section $\mathbf{8 . 3}$, your written protocol and the preamble consent. In the preamble consent, please add a sentence at the end of the first paragraph indicating the number of subjects that will be invited to participate.

Please note: Consent and assent forms no longer have an expiration date stamped on them. The consent/assents expire if the study lapses in IRB approval. Enrollment cannot take place if a study lapses in approval. For additional information view Guide 038.

Continuation Review Requirements 
You are responsible for submitting a continuation review 30 days prior to the expiration date of your research study. Investigators who allow their study approval to expire have committed significant non-compliance with federal regulations. Such lapses may require reporting to federal agencies, a program audit by compliance auditors to ensure that subjects were not enrolled during the expired period, and may lead to findings of serious and continuing noncompliance if expiration were to occur a second time.

This action will be reported promptly to the IRB at a scheduled full Board meeting.

For guidance on using iRIS, including finding your approved stamped documents, please follow the instructions at http://louisville.edu/research/humansubjects/iRISSubmissionManual.pdf

If you have any questions, please contact the IRB analyst listed above or the Human Subjects Protection Program office at hsppofc@louisville.edu.

$$
\text { M. Lrensoluin }
$$

Melissa Evans Andris, PhD, Vice Chair

Social/Behavioral/Education Institutional Review Board

MEA/jsp

We value your feedback. Please let us know how you think we are doing: https://www.surveymonkey.com/r/CCLHXRP 


\section{APPENDIX B}

\section{RECRUITMENT EMAIL SCRIPT}

UofL Institutional Review Board IRB NUMBER: 17.1039

A qualitative study of millennial resistance to outmigration in Appalachian Kentucky Short Title: Staying in Appalachia

Thomas Lawson \& Jeanelle Sears

Kent School of Social Wok

\section{Basic Email Script for Participant Recruitment}

Date:

Dear

I am writing to you with knowledge of your/your organization's work on the issue of in Appalachian Kentucky. I would like to invite you/members of your organization to participate in a research study I am conducting for my doctoral dissertation at the University of Louisville, Kent School of Social Work, under the guidance of my dissertation chair and primary investigator Dr. Thomas Lawson. Participation in the study requires either an in-person or phone based interview lasting up to 90 minutes. I will be personally conducting all interviews.

Our primary research questions are: 1) What are the experiences of young adults staying in Appalachian Kentucky while working on social, economic, and environmental issues? 2) What meaning do these young people make of their experiences personally, professionally, and within the context of larger regional issues? and 3) What strategies do these young adults utilize to resist the pull toward destinations outside the region and to cope with the challenges of remaining?

I have attached our consent documents for your consideration, as they provide more details about our research process, inclusion criteria, and contact information for individuals associated with this project at the University of Louisville. You may also contact me by the phone number or email listed below.

I hope that you will take this opportunity to share your experiences for the purposes of research and/or to share this opportunity with young adults in your organization. For further questions about what the research involves or to schedule and interview, please contact me by the phone number or email address listed below.

I look forward to hearing about your experiences.

Sincerely,

Jeanelle S. Sears, MSc, MSW

Doctoral Student/Research Manager

Kent School of Social Work

University of Louisville

jeanelle.sears@1louisville.edu

802-488-4403 


\section{RECRUITMENT FLIER}

UofL Institutional Review Boards

\section{Participate in Research!}

You are being invited to participate in a research study conducted at the University of Louisville. The purpose of this study is to explore the connections between issues of development and outmigration and we want to know about the experiences of young adults in Appalachian Kentucky.

There are no known risks for your participation in this research study. The information collected may not benefit you directly. However, the information learned in this study may be helpful to others. This research has approval from the University of Louisville Institutional Review Board.

To participate, you would agree to an audio recorded interview, either in person or by phone. In person interviews will be arranged at a location of your convenience. The interview will take approximately 90 minutes to complete.

For this study, we want to speak with young people who:

1) were born between 1980 and 2000,

2) have lived in Appalachian Kentucky for at least 15 years,

3) are currently living in Appalachian Kentucky,

4) have earned or are currently pursuing a college degree, and

5) engage in volunteer or paid work with an organization that focuses on social, environmental, and/or economic issues locally.

If you are interested, please contact Jeanelle or Tom for more information.

Jeanelle S. Sears, MSc, MSW

Patterson Hall

University of Louisville

Louisville, KY 40292

jeanelle.sears@louisille.edu

802-488-4403
Thomas Lawson, PhD

Patterson Hall

University of Louisville

Louisville, KY 40292

thomas.lawson@louisville.edu

502-852-6922

VERSION 11/13/17 


\section{APPENDIX D}

\section{STATEMENT OF CONSENT}

UofL Institutional Review Boards IRB NUMBER: 17.1039

IRB APPROVAL DATE: 11/21/2017 IRB EXPIRATION DATE: 11/20/2018

\section{A qualitative study of millennial resistance to outmigration in Appalachian Kentucky}

Short Title: Staying in Appalachia

Date

Dear

You are being invited to participate in a research study by answering interview questions about young adult experiences in Appalachian Kentucky. The purpose of this study is to explore the connections between issues of development and outmigration through the experiences of young adults in Appalachian Kentucky. The primary research questions are: 1) What are the experiences of young adults staying in Appalachian Kentucky while working on social, economic, and environmental issues? 2) What meaning do these young people make of their experiences personally, professionally, and within the context of larger regional issues? and 3) What strategies do these young adults utilize to resist the pull toward destinations outside the region and to cope with the challenges of remaining?

This study is conducted by Dr. Thomas Lawson of the University of Louisville ( $U$ of $L$ ) and his doctoral student Jeanelle Sears, Msc, MSW. There are no known risks for your participation in this research study. The information collected may not benefit you directly. The information learned in this study may be helpful to others. The information you provide will be used for the purpose of the student's doctoral dissertation, but could also be used in future publications or in presentations to communities or organizations interested in the findings. Your interview will be audio recorded and then coded without your name and stored in a password protected Dropbox folder as well as in a password protected web based data analysis program called Dedoose ${ }^{\mathrm{TM}}$. The interview will take approximately 90 minutes to complete.

Individuals from the Kent School of Social Work at $U$ of $L$, the Institutional Review Board (IRB), the Human Subjects Protection Program Office (HSPPO), and other regulatory agencies may inspect these records. In all other respects, however, the data will be held in confidence to the extent permitted by law. Should the data be published, your identity will not be disclosed.

Taking part in this study is voluntary. By answering interview questions, you agree to take part in this research study. You do not have to answer any questions that make you uncomfortable. You may choose not to take part at all. If you decide to be in this study you may stop taking part at any time.

If you have any questions, concerns, or complaints about the research study, please contact: Dr. Thomas Lawson 502-852-6922

If you have any questions about your rights as a research subject, you may call the Human Subjects Protection Program Office at (502) 852-5188. You can discuss any questions about your rights as a research subject, in private, with a member of the Institutional Review Board (IRB). You may also call this number if you have other questions about the research, and you cannot reach the research staff, or want to talk to someone else. The IRB is an independent committee made up of people from the 


\title{
CURRICULUM VITAE
}

\author{
Jeanelle Susanne Sears \\ jeanelle.sears@louisville.edu \\ 1010 Valley Bluff Dr., Apt. 1 \\ Perrysburg, $\mathrm{OH} 43551$ \\ 802-488-4403
}

\section{EDUCATION}

PhD, Social Work, ABD, anticipated graduation May 2019

University of Louisville, Louisville, KY

MSW, Social Work, 2011

University of Vermont

MSc, Cross Cultural Studies of Childhood, Child Development and Youth, 2009 Brunel University, London, United Kingdom

Graduate Diploma, Forced Migration and Refugee Studies, 2006 The American University in Cairo, Egypt

BA, Anthropology and Psychology, 2005

University of Louisville, Louisville, KY

\section{RESEARCH EXPERIENCE}

Qualitative Analyst: The Afya Project, Dr. Jelani Kerr

Collaborated with public health researchers for coding focus group data using computer assisted analysis software. The Afya Project seeks to increase knowledge, access, and use of Pre-exposure prophylaxix (PrEP) among individuals at risk of contracting HIV. University of Louisville, School of Public Health and Information Sciences. (February, 2019)

Research Manager: Sobriety Treatment and Recovery Teams Program Evaluation (S.T.A.R.T.),Dr. Martin Hall

Trained, supervised and assisted in primary data collection, collaborated with team members to track, analyze and report outcomes, prepared IRB documents, academic manuscripts, and organizational reports as well as developed and delivered professional presentations. University of Louisville, Kent school of Social Work, Louisville, KY. (August 2014-June 2018)

Research Assistant: Disparities in health, and life experience among foreign-born older adults and their family caregivers, Dr. Hee Hyul Moon

Conducted literature review and produced literature database and summaries, assisted with data analysis, manuscript preparation, and development of conference presentations. Kent School of Social Work, Louisville, KY. (September 2016-January 2018)

Project Manager: Despensa de alimentos y hogar de assistencia medica preventiva (The Food Pantry preventative medical home without walls) pilot project, Dr. Anna Faul

Trained, supervised, and coordinated activities of lay community health workers (promotoras), social work students, and volunteers. Program direction 
included healthy lifestyles education, on site biomarker testing, care and coordination, and data collection and management. Institute for Sustainable Health and Optimal Ageing, Louisville, KY. (June 2015-December 2016)

\section{Participatory Action Researcher: Refugee Youth, Lucy Pearson}

Completed 6 months of participant observation and participatory action research strategies, alongside researching, writing, editing, designing, and publishing a book entitled, Becoming a Londoner. The purpose of the book was to describe the experiences of young refugees in London and to share with service providers the best practices in service delivery from a youth perspective. London, UK. (December 2008-June 2009)

Data Collector, Department of Psychological and Brain Sciences, Dr. Suzanne Meeks

Administered primary data surveys and conducted observational coding of behaviors among participants in a study evaluating a psychological intervention for depression among older adults in assisted living facilities in Louisville. University of Louisville, KY. (August 2003-May 2005)

\section{PRACTICE EXPERIENCE}

\section{Associate Director of Health and Safety, Berea College Promise Neighborhood}

Developed, evaluated, and supervised grant programming in the areas of wellness and safety across three Eastern Kentucky counties. Managed related funding in the amount of over $\$ 3$ million. Served as a member of the core leadership team within the organization. Direct program responsibilities included the direction of Recovery Coaching, Sexual Assault and Violence Prevention, and Farm to School programming. Berea, KY. (January 2013-July 2014)

\section{Social Worker, Vermont Department for Children and Families}

Child protection and juvenile delinquency case work including safety and case planning, home visits, risk assessments, on call work, monitoring child placements, and representing the department in court and other community settings. Barre, VT. (May 2011-November 2012)

\section{Trainee, Vermont Department for Children and Families}

Conducted casework through juvenile and family cases as assigned and cofacilitation of a voluntary parenting group within a partner organization. Middlebury, VT. (September 2010-May 2011)

\section{Social Work Intern, Bellows Free Academy and Common Ground Teen Center}

Counseled high school students, assisted in Drop-Out Prevention research, cofacilitated group-work both within the school and the teen center, and collaborated with school staff to prepare a district evaluation report. St. Albans, VT. (September 2009-May 2010) 


\section{Program Director, Corbin Housing Authority}

Designed and facilitated the direct provision of summer youth programming for the local public housing developments, coordinated resources and transportation, handled administrative duties, and combined these roles with family outreach. Corbin, KY (June-July 2009)

\section{Program Director, Arcadia Community Center}

Worked closely with children and families through case management, advocacy, community organizing, directed center activities, wrote and managed grants, raised funds and supervised staff and volunteers while living on site. Louisville, KY (August 2006-August 2008)

\section{AWARDS AND RECOGNITION}

2019 University of Louisville Graduate Dean's Citation

2018 University of Louisville Doctoral Dissertation Completion Award

2017 U.S. Department of State Critical Language Scholarship, Bangla

2015 Community Engaged Research Academy Scholar, University of Louisville

2014 Idea Festival Fellow, University of Louisville

2010 Title IV-E Child Welfare Traineeship Recipient, State of Vermont

2009-2011 Graduate Fellow, University of Vermont

2008 Induction, Honorable Order of Kentucky Colonels

2008 Mary Churchill Humphrey Centenary Memorial Scholarship, University of

Louisville

2005 U.S. Fulbright Student Award, Institute for International Education, Egypt

2005 Anthropology Merit Award, University of Louisville

2005 University Honors Scholar, University of Louisville

2003 Bonner Leader of the Year, University of Louisville

\section{PROFESSIONAL MEMBERSHIPS}

2016-Present Appalachian Studies Association

2015-Present Society for Social Work and Research

2017 Council on Social Work Education

2009-2012 National Association of Social Workers

\section{$\underline{\text { SERVICE }}$}

Service to the Kent School of Social Work

Tutor to MSW student, 2016

Judge for BSW Capstone Poster Session, 2015

Service to the University of Louisville

Appointed Member of University of Louisville Sustainability Council, 2016-7

Advisor to Fulbright Applicants and Selection Committee, 2015-7

Doctoral Student Advisor to Community Engaged Research Academy Committee, 2015

Service to the Community

Research and writing support to FrackFree Foothills regarding the potential physical and social impacts of hydraulic fracturing in Kentucky, 2015 
Service to Social Work

Manuscript editor for Serbian child welfare scholar Emina Borjanic, 2018.

First author of the American Academy of Social Work and Social Welfare Grand

Challenges Policy Initiative and Action Statement: Environmental Change and

Displacement, 2017

\section{PEER REVIEWED PUBLICATIONS}

\section{Manuscripts in Press or Published}

Moon, H., Badana, A.N.S., Sears, J.S., \& Haley, B. (2018). Dementia Prevalence in Older Adults: Variation by Race/Ethnicity and Immigrant Status. American Journal of Geriatric Psychiatry. (In Press)

Willet, J. \& Sears, J. S. (2018). Social Work Research at the Intersection of Environmental Change and Human Movement: Lessons from Kenya. International Social Work, 34(5), 558-572, DOI: 10.1080/02615479.2015.1066326.

Hall, M. T., Ball, D., Sears, J. S., Higgins, G. E., Logan, TK, \& Golder, S. (2018). Past-Year Nonmedical Use of Prescription Drugs among Criminal Justice-Involved Women: The Role of Post Traumatic Stress Disorder. Substance Abuse. (In Press)

Sears, J.S., Verbist, A. N., Wilfong, J., Thomas, T. A., Mount, S., \& Sabree, R., \& van Zyl, M. A. (2018). Mapping the science of social work debate: An exercise in doctoral student education. Journal of Social Work Education. (In Press)

Sears, J. S., Hall, M. T., Harris, L. M., Mount, S., Willauer, T., Posze, L., \& Smead, E. (2017). "Like a Marriage": Partnering with Peer Mentors in Child Welfare. Children \& Youth Services Review, 74, 80-86.

Hall, M. T., Huebner, R. A., Sears, J. S., Posze, L., Willauer, T. \& Oliver, J. (2015). Sobriety Treatment and Recovery Teams in Rural Appalachia: Implementation and Outcomes. Child Welfare, 94(4), 119-138.

\section{Manuscripts in Progress}

Hall, M. T., \& Sears, J. S. Theories of nonmedical prescription drug use: A systematic review.

Hall, M.T. \& Sears, J.S. Applications of Motivational Interviewing in Child Welfare.

Moon, H., Sears, J.S., \& Rote, S. Differences in health and well-being between caregivers of native born and foreign born older adults in the United States.

Moon, H. \& Sears, J.S. Grief among foreign born dementia caregivers: A systematic review. 
Sears, J. S. \& Sossou, M. A. Environmental Change and Human Movement: Developing Next Steps for Social Work's Grand Challenges.

Sears, J. S. \& Joseph, D. Climate Change in Small Island Caribbean States: Implications for Social Work

Ayangeakaa, S.D., Kerr, J.C., Combs, R.M., Harris, L., Sears, J., Northington, T., Parker, K., Sterrett-Hont, E., \& Krigger, K. A novel approach to HIV prevention: Examining view of Pre-Exposure Prophylaxis (PrEP) among Young African Americans in Louisville, Kentucky

\section{POSTERS \& PRESENTATIONS}

Ayangeakaa, S.D., Kerr, J.C., Combs, R.M., Harris, L., Sears, J., Northington, T., Parker, K., Sterrett-Hont, E., \& Krigger, K. (2019). A novel approach to HIV prevention: Examining view of Pre-Exposure Prophylaxis (PrEP) among Young African Americans in Louisville, Kentucky, KY3. Poster accepted for the Kentucky Public Health Conference. Covington, KY.

Moon, H., Badana, A.N.S., Sears, J.S., \& Haley, B. (2019). Dementia Prevalence in Older Adults: Variation by Race/Ethnicity and Immigrant Status. Poster accepted for the Society for Social Work Research Annual Conference. San Francisco, CA.

Sears, J.S. \& Moon, H. (2018). Differences in physical health and psychological well-being among caregivers of older adults in the United States: Does nativity status matter? Poster accepted for the Society for Social Work Research Annual Conference. Washington, D.C.

Sears, J. S., Hall, M. T., Harris, L. M., Mount, S., Willauer, T., Posze, L., \& Smead, E. (2017). "Like a Marriage": Partnering with Peer Mentors in Child Welfare. Poster presented at the Society for Social Work Research Annual Conference. New Orleans, LA.

Willauer, T., Sears, J. S., \& Avery, S. (2016). Integrating Peer Support Specialists in Child Welfare: Lessons from Sobriety Treatment and Recovery Teams (START). Workshop presented at The National Conference on Child Abuse and Neglect. Washington, D.C.

Hall, M. T., Posze, L., Willauer, T., Huebner, R. Sears, J. S., \& Maher, E. (2016). Successful substance abuse, and therapeutic program elements for young children and their families in child welfare: The Sobriety Treatment and Recovery Teams Program. Presented at the Child Welfare League of America National Conference. Orange County, California.

Sears, J.S. \& Morgan, S. (2016). Despensa de alimentos y hogar de assistencia medica preventiva/ The food pantry preventative medical home without walls: A pilot in sustainable health. Poster presented at the Optimal Ageing Conference. Louisville, Kentucky. 
van Zyl, M. A., Verbist, A.N., Sears, J.S., Thomas, T.A., Wilfong, J., Mount, S., \& Sabree, R. (2016). The Science of Social Work Debate. Poster presented at the Society for Social Work and Research Annual Conference. Washington D.C.

Sears, J. S. \& Wilfong, J. (2015). Including the Natural Environment in Social Work Education. Roundtable discussion facilitated at the Kentucky Association of Social Work Educators Conference. Frankfort, KY.

\section{MANUSCRIPT REVIEWS}

Journal of the Society for Social Work Research (2016 [1])

Journal of Sociology and Social Welfare (2017 [2])

Child and Family Social Work (2018 [1])

Perspectives in Social Work (2019 [1])

\section{TEACHING EXPERIENCE}

University of Louisville, Kent School of Social Work

Online Instructor, Advanced Social Work Practice II: Fall 2017, Fall 2018

Online Instructor, Advanced Social Work Practice III: Spring 2018

Teaching Assistant, Issues in Policy and Service Delivery, Spring 2016

Bowling Green State University, Department of Human Services

Classroom Instructor, Social Welfare Institutions: Fall 2018

Classroom Instructor, Research Methods for the Human Services: Fall 2018 